\title{
Hierarchical Matrix Operations on GPUs
}

\author{
Dissertation by \\ Wajih Halim Boukaram \\ In Partial Fulfillment of the Requirements
}

For the Degree of

Doctor of Philosophy

King Abdullah University of Science and Technology

Thuwal, Kingdom of Saudi Arabia

April, 2020 


\section{EXAMINATION COMMITTEE PAGE}

The dissertation of Wajih Halim Boukaram is approved by the examination committee.

Committee Chairperson: Prof. David E. Keyes

Committee Members: Prof. George Turkiyyah, Prof. Markus Hadwiger, Prof. David Ketcheson, Prof. Eric Darve 
(C)April, 2020

Wajih Halim Boukaram

All Rights Reserved 


\section{ABSTRACT \\ Hierarchical Matrix Operations on GPUs Wajih Halim Boukaram}

Large dense matrices are ubiquitous in scientific computing, arising from the discretization of integral operators associated with elliptic PDEs, Schur complement methods, covariances in spatial statistics, kernel-based machine learning, and numerical optimization problems. Hierarchical matrices are an efficient way for storing the dense matrices of very large dimension that appear in these and related settings. They exploit the fact that the underlying matrices, while formally dense, are data sparse. They have a structure consisting of blocks many of which can be well-approximated by low rank factorizations. A hierarchical organization of the blocks avoids superlinear growth in memory requirements to store $n \times n$ dense matrices in a scalable manner, requiring $O(n)$ units of storage with a constant depending on a representative rank $k$ for the low rank blocks. The asymptotically optimal storage requirement of the resulting hierarchical matrices is a critical advantage, particularly in extreme computing environments, characterized by low memory per processing core. The challenge then becomes to develop the parallel linear algebra operations that can be performed directly on this compressed representation. In this dissertation, I implement a set of hierarchical basic linear algebra subroutines (HBLAS) optimized for GPUs, including hierarchical matrix vector multiplication, orthogonalization, compression, low rank updates, and matrix multiplication. I develop a library of open source batched kernel operations previously missing on GPUs for the high performance implementation of the $\mathcal{H}^{2}$ operations, while relying wherever possible on existing open source and vendor kernels to ride future improvements in the technology. Fast marshaling routines extract the batch operation data from an efficient representation of the trees 
that compose the hierarchical matrices. The methods developed for GPUs extend to CPUs using the same code base with simple abstractions around the batched routine execution. To demonstrate the scalability of the hierarchical operations I implement a distributed memory multi-GPU hierarchical matrix vector product that focuses on reducing communication volume and hiding communication overhead and areas of low GPU utilization using low priority streams. Two demonstrations involving Hessians of inverse problems governed by PDEs and space-fractional diffusion equations show the effectiveness of the hierarchical operations in realistic applications. 


\section{ACKNOWLEDGEMENTS}

I would like to thank my advisor Professor David Keyes for his constant support of my research, understanding of my work habits, and encouragement to keep pushing through. I greatly admire his positivity and ability to turn a phrase and his dedication to the field has been an inspiration to me. Words are not enough (especially mine!), but they will have to do: Thank you from the bottom of my heart.

I have worked with my co-advisor, Professor George Turkiyyah from my alma mater, the American University of Beirut, for a decade and I would like to thank him for all his patience and time, the innumerable talks we had over the years, his mentorship and guidance and inexhaustible drive. This work would not have been possible without him.

I would like to wholeheartedly thank the members of my committee, Professor Markus Hadwiger, Professor David Ketcheson and Professor Eric Darve, for their comments on and suggestions for my thesis and for making the time to attend the defense.

I would also like to thank my colleagues at the Extreme Computing Research Center at KAUST and the KAUST Supercomputing Lab for their help running the distributed algorithms on the IBEX cluster. Special thanks to Lisandro Dalcin for his software engineering prowess and Stefano Zampini for his tireless efforts to run the code on the SUMMIT supercomputer and incorporating the code into the PETSC library.

Finally, I would like to thank KAUST for providing all the support and comfort I could ever need to finish my research and my friends and family for supporting my decision to move to KAUST to pursue my $\mathrm{PhD}$. 


\section{TABLE OF CONTENTS}

\begin{tabular}{|l} 
Examination Committee Page \\
\hline
\end{tabular}

$\begin{array}{ll}\text { Copyright } & 3\end{array}$

\begin{tabular}{ll}
\hline Abstract & 4
\end{tabular}

\begin{tabular}{|l|l}
\hline Acknowledgements & 6
\end{tabular}

\begin{tabular}{ll}
\hline List of Abbreviations & 11
\end{tabular}

\begin{tabular}{ll}
\hline List of Figures & 12
\end{tabular}

\begin{tabular}{ll}
\hline List of Tables & 19
\end{tabular}

\begin{tabular}{ll}
\hline List of Algorithms & 20
\end{tabular}

$\begin{array}{lll}1 & \text { Introduction } & 22\end{array}$

1.1 Hierarchical Matrices . . . . . . . . . . . . . . . . . . . . . . . 23

1.1.1 Structure of an $\mathcal{H}$-matrix: Hierarchy of Blocks . . . . . . . . . 23

1.1.2 Structure of an $\mathcal{H}^{2}$-matrix: Hierarchy of Bases . . . . . . . . . 25

1.2 Related Work . . . . . . . . . . . . . . . . . . . . . . . . 28

1.3 Thesis Outline . . . . . . . . . . . . . . . . . . . . . . . . . . . . . . . . 29

2 Efficient Hierarchical Matrix Operations on GPUs 32

2.1 GPU Considerations . . . . . . . . . . . . . . . . . . . . 33

2.1 .1 GPU Kernels . . . . . . . . . . . . . . . . . . . . . . . . . . . 33

$2.1 .2 \quad \mathrm{Warps} \ldots \ldots \ldots \ldots 33$

2.1 .3 Memory . . . . . . . . . . . . . . . . . . . . . . 34

2.2 Architectural Challenges . . . . . . . . . . . . . . . . . . . . . 35

2.3 Flattening the Trees for Efficient GPU Marshaling . . . . . . . . . . . 36

3 Batched Linear Algebra Subroutines 38

3.1 Background . . . . . . . . . . . . . . . . . . . . . . . 38 
3.1 .1 QR Factorization . . . . . . . . . . . . . . . . . . . . . . 38

3.1 .2 SVD Algorithms . . . . . . . . . . . . . . . . . . . . . . . . . 39

3.1.3 $\quad$ Adaptive Randomized Approximation . . . . . . . . . . . . . . 41

3.2 Batched QR Decomposition . . . . . . . . . . . . . . . . . . . . . . . 44

3.2.1 Implementation . . . . . . . . . . . . . . . . . . . . . . . . 44

3.2 .2 Performance . . . . . . . . . . . . . . . . . . 45

3.3 Register Memory One-Sided Jacobi . . . . . . . . . . . . . . . . . . . 47

3.3 .1 Implementation . . . . . . . . . . . . . . . . 47

3.3 .2 Performance . . . . . . . . . . . . . . . . . . . . . . . . 48

3.4 Shared Memory One-Sided Jacobi . . . . . . . . . . . . . . . . . . . . 49

3.4 .1 Implementation . . . . . . . . . . . . . . . . . . . . . . . . . . 49

3.4 .2 Performance . . . . . . . . . . . . . . . . . . . 50

3.5 Global Memory One-Sided Block Jacobi . . . . . . . . . . . . . . . . 51

3.5.1 Gram Matrix Block Jacobi SVD . . . . . . . . . . . . . . . . . 53

3.5 .2 Direct Block Jacobi SVD . . . . . . . . . . . . . . . . . . 54

3.5 .3 Performance . . . . . . . . . . . . . . . . . . 55

3.6 Fixed Rank Randomized SVD . . . . . . . . . . . . . . . . . . . . . 56

3.6.1 Implementation . . . . . . . . . . . . . . . . . . . . . 57

3.6 .2 Performance . . . . . . . . . . . . . . . . . . . . . . 58

3.7 Adaptive Randomized Approximation . . . . . . . . . . . . . . . . . . 59

3.7 .1 Blocked version . . . . . . . . . . . . . . . . . . . . 60

3.7 .2 Implementation . . . . . . . . . . . . . . . . . . . . . 61

3.7 .3 Performance . . . . . . . . . . . . . . . . . . . . 62

3.8 Miscellaneous Batched Operations . . . . . . . . . . . . . . . 65

4 Hierarchical Matrix Vector Multiplication 68

4.1 Upsweep Phase . . . . . . . . . . . . . . . . . . . . . . . . . . . 69

4.2 Multiplication Phase . . . . . . . . . . . . . . . . . . . . . . . . 71

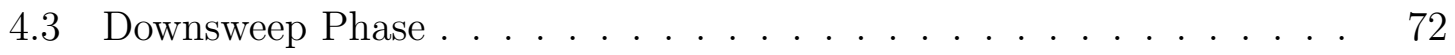

4.4 Kernel Streaming . . . . . . . . . . . . . . . . . . . . . 74

4.5 Performance Results . . . . . . . . . . . . . . . . . . . . . . 75

5 Orthogonalization and Compression 80

5.1 Overview . . . . . . . . . . . . . . . . . . . . . . 80

5.2 Basis Orthogonalization . . . . . . . . . . . . . . . . . 83

5.3 Basis Generation . . . . . . . . . . . . . . . . . . . . . 86

5.4 Basis Truncation . . . . . . . . . . . . . . . . . . . . . . . . . . . . . 89 
5.5 Performance $\ldots \ldots \ldots \ldots \ldots$

$\begin{array}{lll}6 & \text { Hierarchical Adaptive Randomized Approximation } & 97\end{array}$

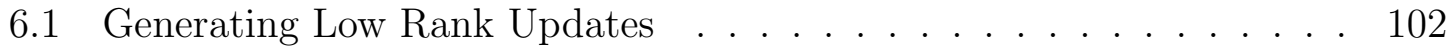

$6.1 .1 \quad$ Sampling Strategy . . . . . . . . . . . . . . . . . . 103

$6.1 .2 \quad$ GPU Implementation . . . . . . . . . . . . . . . . . . 105

6.2 Applying Low Rank Updates. . . . . . . . . . . . . . . . . 106

6.2 .1 Updating the Basis Nodes . . . . . . . . . . . . . . . 107

6.2 .2 Updating the Coupling Nodes . . . . . . . . . . . . . . 108

6.2 .3 GPU Implementation . . . . . . . . . . . . . . . . . . . . . . 109

6.3 Compression to Reduce Rank . . . . . . . . . . . . . . . . . . . 109

6.4 Numerical Experiments . . . . . . . . . . . . . . . . . . . . . . . . . 111

6.4 .1 Construction of a Spatial Statistics Covariance Matrix . . . . 112

6.4 .2 Hierarchical Matrix-matrix Multiplication . . . . . . . . . . 114

6.4 .3 Schur Complement Computation . . . . . . . . . . . . 117

7 Iterative Methods for $\mathcal{H}^{2}$-matrix Inversion 123

7.1 Newton-Schulz Iterations with Hierarchical Matrices . . . . . . . . . . 124

7.2 Higher Order Methods for Faster Convergence . . . . . . . . . . . . 127

7.3 Unrolling Iterations $\quad \ldots \ldots \ldots \ldots$

8 Distributed Hierarchical Matrix Operations 130

8.1 Distributed Construction . . . . . . . . . . . . . . . . . . . . 130

8.2 Distributed Hierarchical Matrix Vector Products . . . . . . . . . . . 133

8.2 .1 Distributed Upsweep . . . . . . . . . . . . . . . . . . . . 134

8.2 .2 Distributed Multiplication . . . . . . . . . . . . . . . . 135

$8.2 .3 \quad$ Distributed Downsweep $\ldots \ldots \ldots \ldots \ldots$

8.2 .4 Communication Overhead . . . . . . . . . . . . . 136

$8.3 \quad$ Optimizing Communication Volume $\ldots \ldots \ldots \ldots \ldots$

$8.3 .1 \quad$ Compressing the Off-diagonal Blocks _ . . . . . . . . . 137

8.3 .2 Optimized Multiplication Phase . . . . . . . . . . . . . . . 139

8.4 Overlapping Communication with Computation . . . . . . . . . . 142

8.5 Scalability Results $\ldots \ldots \ldots \ldots \ldots$

9 Application: Hessians Arising in Inverse Problems Governed by $\begin{array}{ll}\text { PDES } & 149\end{array}$

9.1 Why do Hessians Admit Hierarchical Low Rank Representations? . . 152 
9.2 Illustrative Applications $\ldots \ldots \ldots \ldots$ 9.2.1 Density Inversion in 1D Time-dependent Diffusion Equation . 157 9.2 .2 Source Inversion in Stationary Advection-diffusion . . . . . . 160

\section{Application: Hierarchical Matrix Approximations for Space-Fractional} Diffusion Equations $\quad 164$

10.1 The Fractional Diffusion Equation and its Particle Approximation . . 166

10.1 .1 The Fractional Laplacian . . . . . . . . . . . . . . 166

10.1 .2 Particle Approximation . . . . . . . . . . . . . . 167

10.2 Interpolatory Construction . . . . . . . . . . . . . . . . . . . 169

10.3 Performance of Hierarchical Matrix Operations. . . . . . . . . . . 172

10.4 Illustrative Simulations . . . . . . . . . . . . . . . . . . . 177

10.4 .1 Transient Solution . . . . . . . . . . . . . . . . . . 178

10.4 .2 Forced Transient and Steady State Solutions . . . . . . . . 182

\begin{tabular}{ll}
\hline 11 Conclusions & 187
\end{tabular}

11.1 Contributions . . . . . . . . . . . . . . . . . . . . 187

11.2 Future Work . . . . . . . . . . . . . . . . . . . . . . . . . . . . . 188

11.3 Conclusion . . . . . . . . . . . . . . . . . . . . . . . . . . . 191

\begin{tabular}{ll}
\hline References & 192
\end{tabular}

\begin{tabular}{ll}
\hline Appendices & 204
\end{tabular} 


\section{LIST OF ABBREVIATIONS}

$\begin{array}{ll}\text { ARA } & \text { Adaptive Randomized Approximation } \\ \text { BGS } & \text { Block Gram Schmidt } \\ \text { BLAS } & \text { Basic Linear Algebra Subroutines } \\ \text { BLR } & \text { Block Low Rank } \\ \text { BSR } & \text { Block Sparse Row } \\ \text { CG } & \text { Conjugate Gradient } \\ \text { CPU } & \text { Central Processing Unit } \\ \text { GEMM } & \text { General Matrix Matrix multiplication } \\ \text { GEMV } & \text { General Matrix Vector multiplication } \\ \text { GPU } & \text { Graphics Processing Unit } \\ \text { GS } & \text { Gram Schmidt } \\ \text { HARA } & \text { Hierarchical Adaptive Randomized Approxi- } \\ & \text { mation } \\ \text { HBLAS } & \text { Hierarchical Basic Linear Algebra Subroutines } \\ \text { HBS } & \text { Hierarchically Block-Separable } \\ \text { HSS } & \text { Hierarchically Semi-Separable } \\ \text { LAPACK } & \text { Linear Algebra PACKage } \\ \text { MUMPS } & \text { MUltifrontal Massively Parallel Sparse solver } \\ \text { NS } & \text { Newton Schulz } \\ \text { PDE } & \text { Partial Differential Equation } \\ \text { RDMA } & \text { Remote Direct Memory Access } \\ \text { SIPG } & \text { Symmetric Interior Penalty Galerkin } \\ \text { SPD } & \text { Symmetric Positive Definite } \\ \text { spMV } & \text { Sparse Matrix Vector } \\ \text { SVD } & \text { Singular Value Decomposition } \\ \text { TLR } & \text { Tile Low Rank } \\ & \end{array}$




\section{LIST OF FIGURES}

1.1 A general partitioning of a matrix with all leaves of the matrix tree

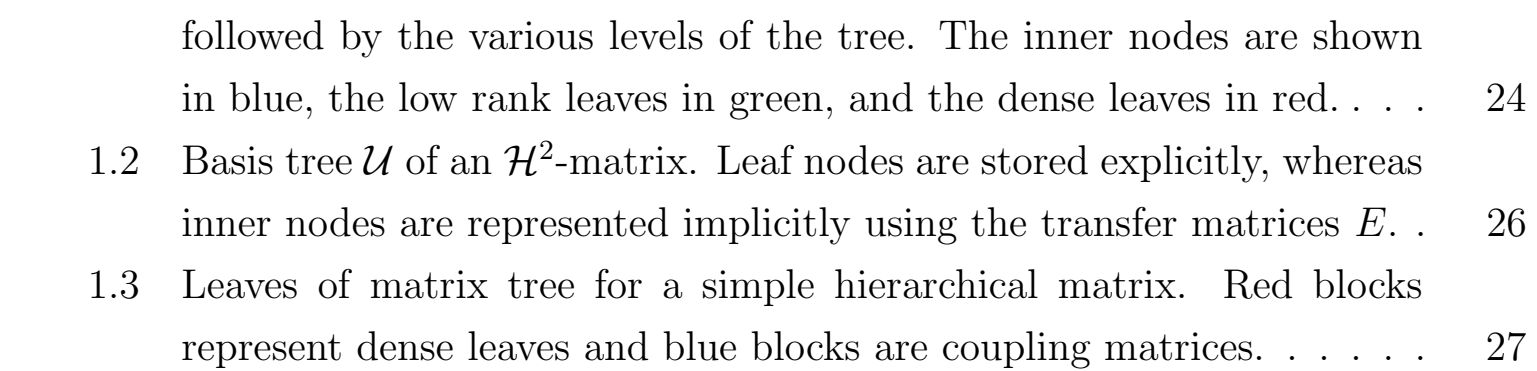

$2.1 \quad$ Kernel launch overhead shown on the execution timeline in orange for

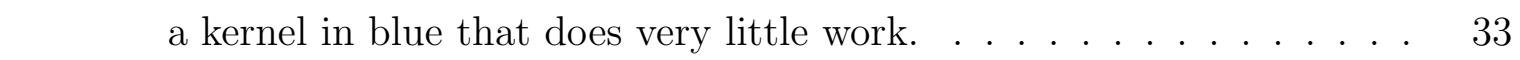

2.2 The memory hierarchy of a modern GPU. . . . . . . . . . . . . . . . 34

2.3 Basis tree structure. Any data associated with the basis tree uses the \begin{tabular}{|cc}
\hline node numbers to locate the position of the node data in memory. . . 37 & 37
\end{tabular}

3.1 Left: matrix rows allocated to thread registers in a warp. Right: par-

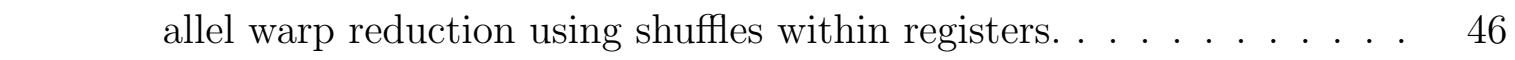

3.2 One step of the QR factorization where a panel $\mathrm{P}$ is factored to produce a triangular factor $\mathrm{R}$ and reflectors $\mathrm{V}$ which are used to update the trailing sub-matrix M. . . . . . . . . . . . . . . . . 46

3.3 Comparing batched QR kernels for 1000 matrices of varying size on a P100 GPU in single and double precision. . . . . . . . . . . . . . 47

3.4 Performance of the batched register memory SVD on a P100 GPU for 1000 matrices of varying size in single and double precision arithmetics. 49

3.5 Distribution of column pairs to warps at each step of a sweep. . . . . 51

$3.6 \quad$ Shared memory kernel implementation details. . . . . . . . . . . . . 52

3.7 Performance of the batched shared memory SVD on a P100 GPU for 1000 matrices of varying size in single and double precision arithmetics. 52

3.8 Profile of the different phases of the block Jacobi SVD for 200 matrices of varying size on a P100 GPU in double precision. Single precision exhibits similar behavior. . . . . . . . . . . . . . . . . . . . 56 
$3.9 \quad$ Batched block Jacobi performance for 200 matrices of varying size on a P100 GPU in single and double precision arithmetics. . . . . . . . 57

3.10 Profile of the different phases of the batched randomized SVD for 200 matrices of varying size on a P100 GPU in double precision. Single precision exhibits similar behavior. . . . . . . . . . . . . . . 59

3.11 Batched randomized SVD performance for 200 matrices of varying size on a P100 GPU in single and double precision for the first 64 singular values and vectors. . . . . . . . . . . . . . . 60

3.12 Batched ARA performance for a precision of $10^{-6} . \ldots \ldots \ldots 63$

3.13 Comparison of batched ARA and SVD kernels . . . . . . . . . 63

4.1 Overview of the hgemv. Computation with the low rank blocks is split \begin{tabular}{|l|}
\hline into three phases: upsweep, multiplication, and downsweep. The up- \\
\hline sweep computes a tree $\hat{x}$ from the input vector $x$ by first projecting it \\
\hline to the basis leaves and then sweeping up the tree using the transfer \\
\hline matrices. $\hat{x}$ is then fed into the block sparse multiplication phase to \\
\hline produce the $\hat{y}$ tree. Finally, the downsweep computes the output vec- \\
\hline tor $y$ by first accumulating partial sums within each level of $\hat{y}$ using \\
\hline the transfer matrices. The leaf level of $\hat{y}$ then contains the full sums \\
\hline in terms of the basis leaves which are expanded to form the output \\
\hline vector $y$ and added to the results of the dense matrix-vector product \\
\hline to produce the final result
\end{tabular}

4.2 The block sparse row storage for a $6 \times 6$ matrix with $2 \times 2$ blocks. . . $\quad 73$

$4.3 \quad$ Overlapping the dense phase of the hgemv with the areas of low GPU

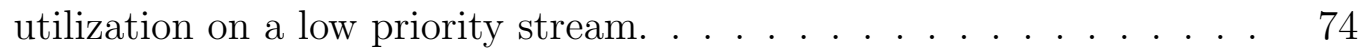

$4.4 \quad$ Structure of the 2D (top) and 3D (bottom) sample covariance matrices used in the examples for $n=2^{14}$, with a zoom on portions of them. Notice that the 3D problem does not have as many large blocks that admit a low rank approximation as the 2D problem and therefore results in a representation that has higher memory demand for the same

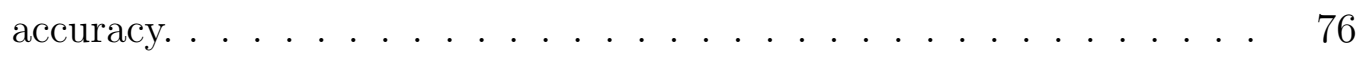

4.5 Breakdown of the hgemv phases in percentages of total operation time in single (left) and double (right) precision on a P100 GPU showing that, for smaller problem sizes where the basis tree has very few levels, the upsweep and downsweep do not fully utilize the hardware. . . . . 77 
4.6 Runtime of hgemv on a single P100 GPU, showing asymptotically linear growth with problem size. Notice that the streamed version that allows overlapping between the dense and the low rank phases of hgemv provides performance boost on small problems. On the larger problems, where the available bandwidth is saturated with the low rank data, the improvement due to overlapping is diminished. . . . . . . . . . . . . . 78

4.7 Achieved bandwidth of hgemv on a single P100 GPU with the streamed kernel achieving up to $78 \%$ of the theoretical bandwidth peak of the GPU. . . . . . . . . . . . . . . . . . 78

5.1 Matrix block row $A_{i}^{q}$ of the low rank portion of the hierarchical matrix \begin{tabular}{|c|}
\hline for the sixth row of the leaf level. $A_{i}^{q}$ includes 5 blocks at level $q$ and \\
\hline sub-blocks coming from higher level block rows. . . . . . . . . . . . . 82
\end{tabular}

5.2 Left: Basis orthogonalization starting from the leaves and sweeping up \begin{tabular}{|c|}
\hline the tree to overwrite the basis with an orthogonal one and generate \\
\hline
\end{tabular}

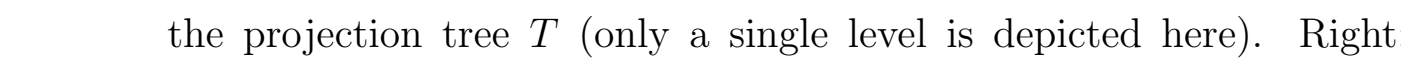
Projection of the leaf level of coupling matrices into a new basis using \begin{tabular}{|c|}
\hline a projection tree $T$. The new basis could be more compact, resulting \\
\hline
\end{tabular}

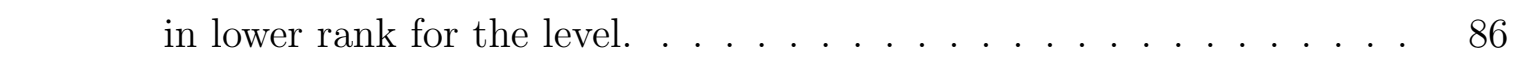

5.3 Constructing $R_{i}^{l}$ from its parent level, the matrix tree row data, and transfer matrices. . . . . . . . . . . . . . . . 88

5.4 Breakdown of the orthogonalization phases in percentages of total operation time in single (left) and double (right) precision on a P100 GPU, showing that the projection phase dominates the runtime. . . . 93

5.5 Runtime and achieved performance of the orthogonalization on a single \begin{tabular}{|c|}
\hline P100 GPU. Note the asymptotic linear growth with problem size. Dou- \\
\hline ble and single precision times are closer to each other than expected
\end{tabular} \begin{tabular}{|l|}
\hline ble and single precision times are closer to each other than expected \\
\hline due to the performance of the cuBLAS batched gemm routines. . . . .
\end{tabular}

5.6 Breakdown of the compression phases in percentages of total operation time in single (left) and double (right) precision on a P100 GPU, showing that the truncation and basis generation phases dominate the computation. .................... 94

5.7 Runtime and achieved performance of compression on a single P100

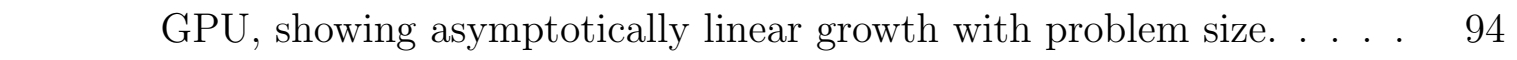


5.8 Effect of the compression on memory and runtime for the 2D and 3D problems, showing significant memory savings for the low rank portion

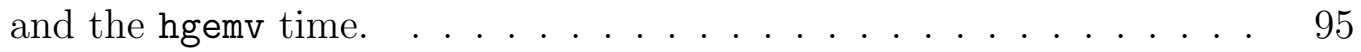

6.1 Examples of the sampling and approximation matrix trees and the application of a generated low rank update of the sampling tree to the approximation tree. . . . . . . . . . . . . . . . . . 100

6.2 The major phases of one level of the hierarchical adaptive randomized approximation. The left figure shows the matrix $A$ that is being approximated with the leaves of the sampling tree overlaid on it, as well as the structured random vectors generated by the sampling tree. These are used to generate the low rank updates of the center figure in a process similar to the ARA. Finally, the updates are applied to the hierarchical matrix $A_{\mathcal{H}}$ of the right figure, where the affected leaves of the matrix tree are highlighted in the shaded areas. . . . . . . . . . 100

6.3 Sampling the second level of the matrix and updating the approximation tree nodes. . . . . . . . . . . . . . . . . . . . . . 100

$6.4 \quad$ Sampling and approximation trees used to construct a hierarchical matrix. . . . . . . . . . . . . . . . . . . . 113

6.5 Scalability and performance of HARA on a representative covariance matrix from spatial statistics. . . . . . . . . . . . . . . . . 114

$6.6 \quad$ Profile showing relative costs of the different phases of the reconstruc-

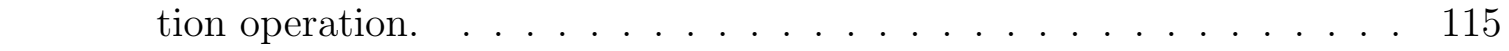

6.7 Comparison of the ranks per level for a problem size of $2^{18}$ for error thresholds of $10^{-2}$ and $10^{-4} . \ldots \ldots \ldots \ldots$

6.8 Overall performance for the construction of the hierarchical matrix on a P100 GPU. . . . . . . . . . . . . . . . . . 116

6.9 Scalability and performance of HARA for constructing an $\mathcal{H}^{2}$ representation of $A^{2}$. . . . . . . . . . . . . . . . . . . 117

6.10 Comparison of the ranks per level when constructing the square of the matrix for a problem size of $2^{18}$ for error thresholds of $10^{-2}$ and $10^{-4}$. 118

6.11 Profile showing relative costs of the different phases of the multiplication operation. . . . . . . . . . . . . . . . . . . . . 118

6.12 Overall performance for the construction of the square of an $\mathcal{H}^{2}$ matrix on a P100. . . . . . . . . . . . . . . . . . . . . . . 119 
7.1 Performance of Hessian inversion on a problem of size $n=16,384$.

\begin{tabular}{|l|}
\hline Number of Hessian-vector products throughout the NS iterations: (a) \\
\hline \hline starting from a scaled identity and (b) a warm starting from previous \\
\hline iteration. (c) Convergence history. . . . . . . . . . . . . . . . . . . . . 126 \\
\hline 7.2 Performance of Hessian inversion using high order iterations of order \\
\hline 8, 16, and 32. . . . . . . . . . . . . . . . . . . . . 128
\end{tabular}

8.1 A hierarchical matrix distributed to 4 GPUs with the block rows of the dense and coupling leaves having the same color residing on the same GPU. The root branch is colored in grey. . . . . . . . . . . . . . . . . 132

8.2 The weak scaling and the profile of the distributed hgemv. . . . . . . 136

8.3 The compressed node data for the off-diagonal block ${ }_{p} \mathcal{A}_{\bar{p}}$ for a block row on process $p$ of a hierarchical matrix distributed to 4 GPUs. Process 2 has no corresponding basis tree data, so it is not included in the list pid . . . . . . . . . . . . . . . . . . . . . . 139

8.4 An example of the execution timeline for the distributed hgemv where the communication time is completely hidden by the processing of the diagonal blocks. . . . . . . . . . . . . . . . . . . . . . . . 142

8.5 The weak scaling performance of the communication optimized distributed hgemv on up to 16 K80 GPUs with ${ }_{p} n=2^{19}$. . . . . . . . . . 143

8.6 Scalability and efficiency of HGEMV on SUMMIT, on two sets of matrices. . . . . . . . . . . . . . . . . . . 148

8.7 Scalability and efficiency of HGEMV on a 64-GPU cluster, on two sets of matrices. . . . . . . . . . . . . . . . . . . . . . . . . . . . . . . . . 148

$9.1 \quad$ (left) Sources $(\bullet)$, receivers (o), and target profile; (right) Hessian structure. . . . . . . . . . . . . . . . . . 157

9.2 (left) Local ranks of Hessian and its inverse. (middle) Convergence history of NS. (right) Number of samples needed to construct the matrix - over the iteratons. . . . . . . . . . . . . . . . 160

9.3 Dependence of the solution for different diffusion parameters $\kappa$ : Shown on the left is the source $m$ entering on the right hand side of (9.11). The right three plots show the solutions of the state equation for $\kappa=$ $10^{-1}, 10^{-2}, 10^{-3}$ (from left to right). Depending on $\kappa$, the problem is diffusion- or advection-dominated. . . . . . . . . . . . . . . . . . . . . 161 
9.4 The $y$-axis shows the number of Hessian-vectors products (samples) needed for constructing hierarchically and globally low rank approximations of the misfit Hessian to an accuracy of $\epsilon=10^{-4}$ for increasing number of observations plotted on the $x$-axis. Shows are results for $\kappa=10^{-1}$ (left), $10^{-2}$ (middle), and $10^{-3}$ (right). . . . . . . . . . . 162

9.5 8-level structure of Hessian (left) and corresponding maximum local

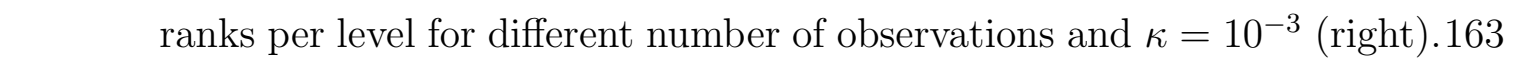

10.1 Clustering produced by a binary $k$-d tree partitioning for a regular (left) and irregular (right) spatial discretization of square and circular regions. The labels $t$ and $s$ identify the clusters that affect a matrix block $A_{t s}$. . . . . . . . . . . . . . . . . . . . . . . . . 170

10.2 Hierarchical Matrix Structure produced by admissibility parameter $\eta=$

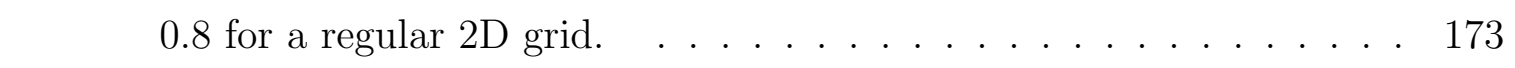

10.3 Performance of matrix construction to an error threshold of $10^{-5}$ on a \begin{tabular}{|c|}
\hline range of problem sizes: (a) CPU time for interpolation and algebraic \\
\hline compression phases; (b) Memory footprint of the dense and low rank \\
\hline
\end{tabular}

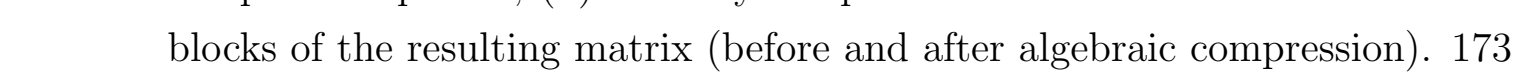

10.4 Impact of the choice of the compression threshold on the memory footprint and maximum block rank of a fractional diffusion hierarchical matrix of size $2^{20}$. . . . . . . . . . . . . . . . . 175

10.5 Performance of the matrix-vector multiplication. . . . . . . . . . . . . 176

10.6 Performance of the order 16 hyperpower iterative inversion. . . . . . . 176

10.7 The convergence behavior of the preconditioned conjugate gradient method using a diagonal preconditioner and the two approximate (regularized) inverse preconditioners. . . . . . . . . . . . . . . . 177

10.8 Fundamental solution at $t_{f}=1.5$ for $\alpha=1.5$. Also plotted are profiles obtained using the full diffusion matrix and its hierarchical approximation; in both cases $N_{x}=161$.

\begin{tabular}{|c|c|c|}
\hline 10.9 L1-error $\varepsilon_{D_{\varepsilon}}$ for different values of $\alpha$ and $h$, as indicated. Plotted \\
are results obtained using the full diffusion matrix (circles) and its
\end{tabular}

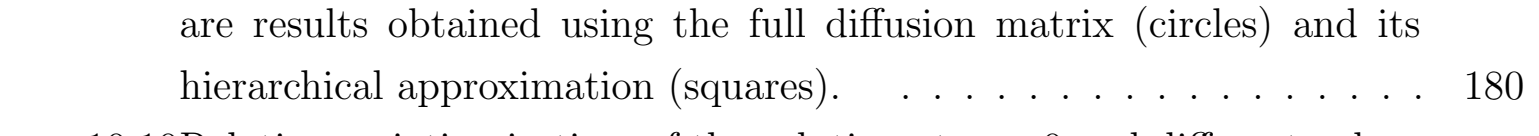

10.10Relative variation in time of the solution at $\mathrm{x}=0$ and different values \begin{tabular}{|l}
\hline of $\alpha$ as indicated. The simulations are performed using $N_{x}=161$ and \\
\hline$\Delta t=2.5 \cdot 10^{-3}$. . . . . . . . . . . . . . . . 183
\end{tabular} 
10.11Profile of the computed solution at $t=64$ along $y=0$. Plotted are curves generated for different values of $\alpha$, as indicated. . . . . . . . . 184

10.12 Profile of the computed solution at $t=64$ along $y=0$. Plotted are curves generated $\alpha=1.5$, and different values of $N_{x}$, as indicated. . . 184 


\section{LIST OF TABLES}

$4.1 \quad$ Notation used for the hierarchical matrix tree algorithms. . . . . . . . 69

5.1 Compression errors for the 2D problem using a truncation threshold of $10^{-7} \ldots \ldots \ldots \ldots . \ldots \ldots 1$

5.2 Compression errors for the 3D problem using a truncation threshold of

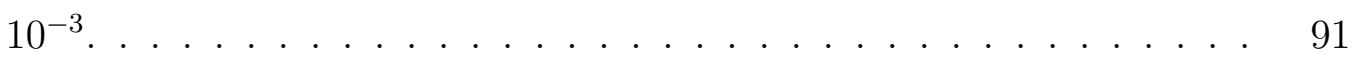

6.1 Results for computing the Schur complement to an accuracy of $\epsilon=10^{-4}$. 119

8.1 Notation used for distributed hierarchical matrix elements. . . . . . . 131

10.1 The memory consumption in GB and memory savings of using a hierarchical representation instead of a dense one. . . . . . . . . . . . . . 174

10.2 Computational cost and memory requirements of the full-matrix and the hierarchical-matrix computations. All the simulations were conducted on the same workstation, comprising 28 physical cores (56 in hyperthreading) on Intel CPU's, and 127 GB of RAM. The codes were designed to run in shared memory parallelism, and compiled using the same compiler. . . . . . . . . . . . . . . . . . . . . . . . . . . 181

10.3 Cost of $A$ and its approximate inverse, and CG iterations, for different

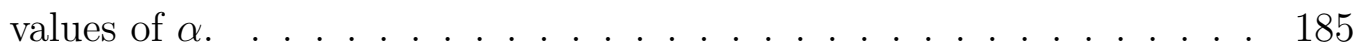




\section{LIST OF ALGORITHMS}

$1 \quad$ Householder $\mathrm{QR} . \ldots \ldots \ldots \ldots$

2 One-sided Jacobi SVD . . . . . . . . . . . . . . . . . . . . . . 40

3 Fixed Rank Randomized Approximation . . . . . . . . . . . . . . . 42

4 Adaptive Randomized Approximation . . . . . . . . . . . . . . 43

5 Batched One-sided Block Jacobi SVD . . . . . . . . . . . . . . . . 55

6 Batched Fixed Rank Randomized SVD . . . . . . . . . . . . . . . 58

7 Batched Adaptive Randomized Approximation . . . . . . . . . . . 67

8 GPU Upsweep Marshaling Routine . . . . . . . . . . . . . . . . . 71

$9 \quad$ GPU Upsweep Algorithm for Forming $\widehat{x}$. . . . . . . . . . . . . 72

10 GPU Matrix Tree Multiplication for $\hat{y} \quad \ldots \ldots \ldots \ldots$

11 GPU Downsweep for Computing $y$. . . . . . . . . . . . . 74

12 GPU Orthogonalization Upsweep Marshaling Routine . . . . . . . . . 85

13 Basis Orthogonalization . . . . . . . . . . . . . . . . 85

14 Projection of Coupling Matrices . . . . . . . . . . . . . . . 87

15 Basis Generation . . . . . . . . . . . . . . . . . . . . . 88

16 GPU Basis Generation Marshaling Routine . . . . . . . . . . . . . . 89

17 Basis Truncation . . . . . . . . . . . . . . . . . . . . . 90

18 Hierarchical Adaptive Randomized Approximation. . . . . . . . . . . 102

19 Hierarchical Adaptive Randomized Approximation. . . . . . . . . . . 121

20 Marshaling Low Rank Update Data . . . . . . . . . . . . . . . . . 122

21 Applying Low Rank Updates to a Hierarchical Matrix . . . . . . . . . 122

$22 \quad$ Stabilized Newton-Schulz for Inverting $A$ Starting with $X_{0} \ldots \ldots$

$23 \quad$ Hierarchical Matrix Iterative Inversion of $A$ Starting with $X_{0} \ldots \ldots$

24 Templated Sampler for Newton-Schulz Iterate, Computes $Y=X_{k+1} \Omega \quad 125$

25 Sampling Hyperpower Iterate $X_{k+1}$ of Order l, Computes $Y=X_{k+1} \Omega$. 128

26 General Admissibility Dual Tree Traversal . . . . . . . . . . . 133

27 Generate the Lists of Remote Interaction Nodes . . . . . . . . . . . 133

28 Generate the Structure of the Distributed Hierarchical Matrix . . . . 134

29 Distributed Upsweep . . . . . . . . . . . . . . . . . . . . . . . . . . 134

30 Distributed Multiplication . . . . . . . . . . . . . . . . . . . 135

31 Distributed Downsweep $\ldots \ldots \ldots \ldots \ldots$ 
$32 \quad$ Compress Receive Nodes $\ldots \ldots \ldots$

$33 \quad$ Exchange Receive and Send Counts . . . . . . . . . . . . . . . . . 139

34 Exchange Receive and Send Lists . . . . . . . . . . . . . . . . . 140

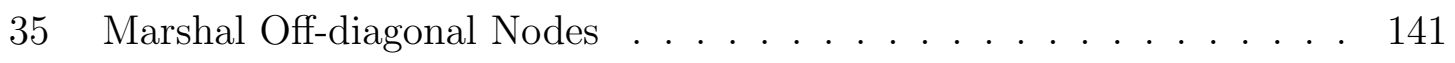

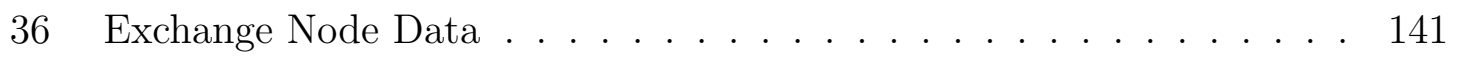

37 Optimized Multiplication Phase . . . . . . . . . . . . . . . . . . . 141 


\section{Chapter 1}

\section{Introduction}

Large dense matrices are ubiquitous in scientific computing. The discretization of integral operators associated with elliptic PDEs results in systems that are dense and on the order of the mesh size. Schur complement methods exploiting dimension reduction in PDE discretizations give rise to large dense systems. Covariance matrices in spatial statistics are dense and on the order of the number of correlated quantities. Kernel-based machine learning algorithms generate large matrices describing pairwise relations between data points. Numerical optimization problems arising in inverse problems, deep learning, or data assimilation are generating ever-more exigent demands for manipulating dense matrices.

Hierarchical matrices are an efficient way for representing the dense matrices of very large dimension that appear in these and related settings. They exploit the fact that the underlying matrices, while formally dense, are data sparse. They have a structure consisting of blocks many of which can be well-approximated by low rank factorizations. Even though the blocks are of varying sizes and locations, treebased data structures can be used to take advantage of the inherent data sparsity and organize the block approximations hierarchically, in effect compressing the dense matrix in an accuracy-controlled manner. This representation can avoid superlinear growth in memory requirements to store $n \times n$ dense matrices in a scalable manner.

The asymptotically optimal storage requirement of the resulting hierarchical matrices is a critical advantage, particularly in extreme computing environments, characterized by low memory per processing core. For many standard applications, the 
compressed hierarchical form produces a few orders-of-magnitude reduction in required memory compared to the equivalent dense representation and makes it possible to fit the matrix in the limited global memory of current generation GPUs, which overcomes the disadvantage of the slow transfer of data between GPU and main memory. The challenge then becomes to develop the parallel linear algebra operations that can be performed directly on this compressed representation. In terms of the operational complexity, many of the linear algebra operations to be performed on hierarchical matrices have linear or log-linear growth as expected from the linear memory growth. Efficient massively parallel implementations of these asymptotically work-optimal operations remain open problems.

\subsection{Hierarchical Matrices}

A great deal of work has been done in the development of representations that exploit the low rank structure of matrix blocks in a hierarchical fashion. Hackbusch [1, 2] pioneered the concepts of hierarchical matrices in the form of $\mathcal{H}$ and $\mathcal{H}^{2}$ matrices as a way to generalize fast multipole methods, and developed a substantial mathematical theory for their ability to approximate integral operators and boundary value problems of elliptic PDEs. These ideas have been developed considerably over the past two decades, for the construction and use of hierarchical matrices in solving discretized integral equations and preconditioning finite element discretizations of PDEs [3, 4, 5, 6, 7]. In the following, I briefly describe the main concepts and representative components of $\mathcal{H}$ and $\mathcal{H}^{2}$ matrices.

\subsubsection{Structure of an $\mathcal{H}$-matrix: Hierarchy of Blocks}

Let $\mathcal{I}$ and $\mathcal{J}$ be the sets of indices of the rows and columns of a matrix, and $T_{\mathcal{I}}$ and $T_{\mathcal{J}}$ be hierarchical clusterings of these index sets, respectively. All blocks within the matrix can then be defined by cluster pairs $(t, s) \in T_{\mathcal{I} \times \mathcal{J}}$. Define an admissible 


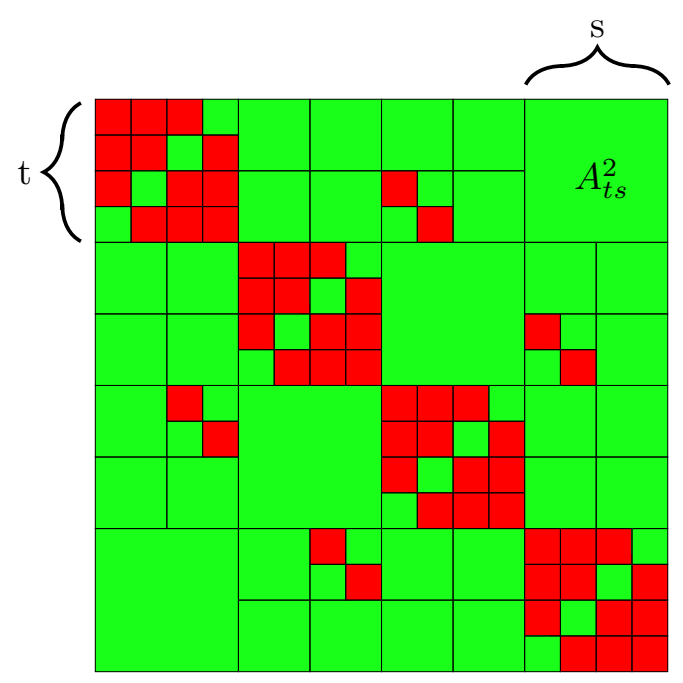

(a) The leaves of the matrix tree for a general partitioning of a matrix. The block $A_{t s}^{2}$ is defined by the labeled cluster pair $(t, s)$ at level 2 of the tree.
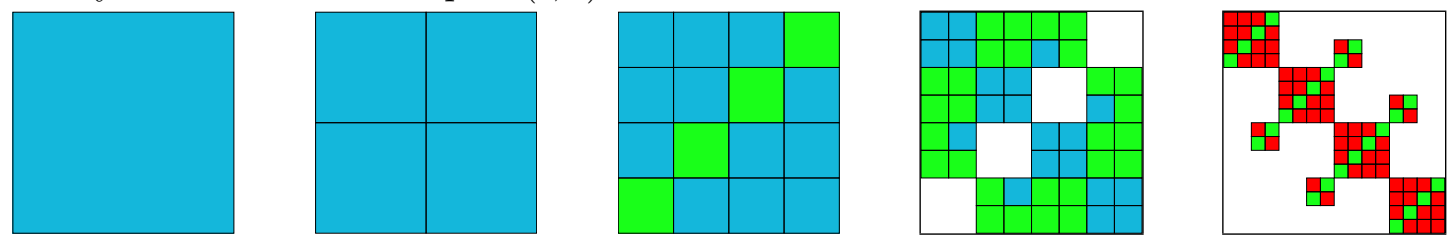

(b) The levels of the matrix tree.

Figure 1.1: A general partitioning of a matrix with all leaves of the matrix tree followed by the various levels of the tree. The inner nodes are shown in blue, the low rank leaves in green, and the dense leaves in red.

block as a block that is either small enough to be stored in dense form or can be well approximated by a low rank matrix. A matrix $A$ in the $\mathcal{H}$-variant of hierarchical matrices can be partitioned into admissible blocks of various sizes that are organized hierarchically as the leaves of a matrix tree. A low rank matrix $A_{t s}^{l}$ on level $l$ of the tree and defined by the cluster pair $(t \times s)$ is represented as the outer product of two tall matrices $B_{t s}^{l}$ and $C_{t s}^{l}$ of rank $k^{l}: A_{t s}^{l}=B_{t s}^{l} C_{t s}^{l}$ or in the orthogonal form $A_{t s}^{l}=U_{t s}^{l} X_{t s}^{l} V_{t s}^{l^{T}}$ where $U_{t s}^{l}$ and $V_{t s}^{l}$ are orthogonal matrices and $X_{t s}^{l}$ is a small $k^{l} \times k^{l}$ matrix. Storage requirements for a problem of size $n$ are $O(n \log (n))$ with a constant dependent on the representative rank $k$ of the low rank blocks. Similarly, algorithmic complexity of operations based on this format vary between $O(n \log (n))$ and $O\left(n \log ^{2}(n)\right)$ 8. Figure $1.1 \mathrm{a}$ shows the leaves of this matrix tree which define 
a complete partitioning of the matrix into blocks, with dense leaves colored red and low rank leaves colored green. Figure $1.1 \mathrm{~b}$ shows the various levels of the tree, where inner nodes are in blue. The generation of the structure of the matrix tree is largely dependent on the application. For example, in the fractional diffusion application of Chapter 10, I explain how to leverage geometric data from the particles generated by a spatial discretization to generate the matrix tree. Other applications might rely on the available algebraic data or various heuristics to determine which blocks of the matrix are admissible as low rank blocks [9]. The ideal structure for a particular application, whether in terms of computational efficiency, memory consumption, communication or even host architecture, is an open problem. This thesis assumes a specified structure and focuses on the development of efficient parallel algorithms for hierarchical operations.

\subsubsection{Structure of an $\mathcal{H}^{2}$-matrix: Hierarchy of Bases}

To avoid the logarithmic factors in the complexity estimates of hierarchical matrices, Hackbusch [10] introduced hierarchical basis $\mathcal{H}$-matrices, calling the resulting representation $\mathcal{H}^{2}$-matrices. To better understand the format, I first define the following:

- The level $A^{l}$ of a matrix tree $A$ contains all blocks $A_{t s}^{l}$ defined by the cluster pairs $(t, s)$ at level $l$.

- The rank $k^{l}$ of a level is the rank of the low rank blocks of $A^{l}$. This rank could be different for each block, but for simplicity I assume all blocks within a level have the same rank. Potential inefficiencies from "over-resolving" the rank of some blocks to accommodate the maximum rank within a given level are mitigated by the efficiency of batched operations that amortize a single kernel call.

- A block row $A_{t *}^{l}$ is the set of leaves within a level $l$ that are defined by the row indexes of cluster $t$. 


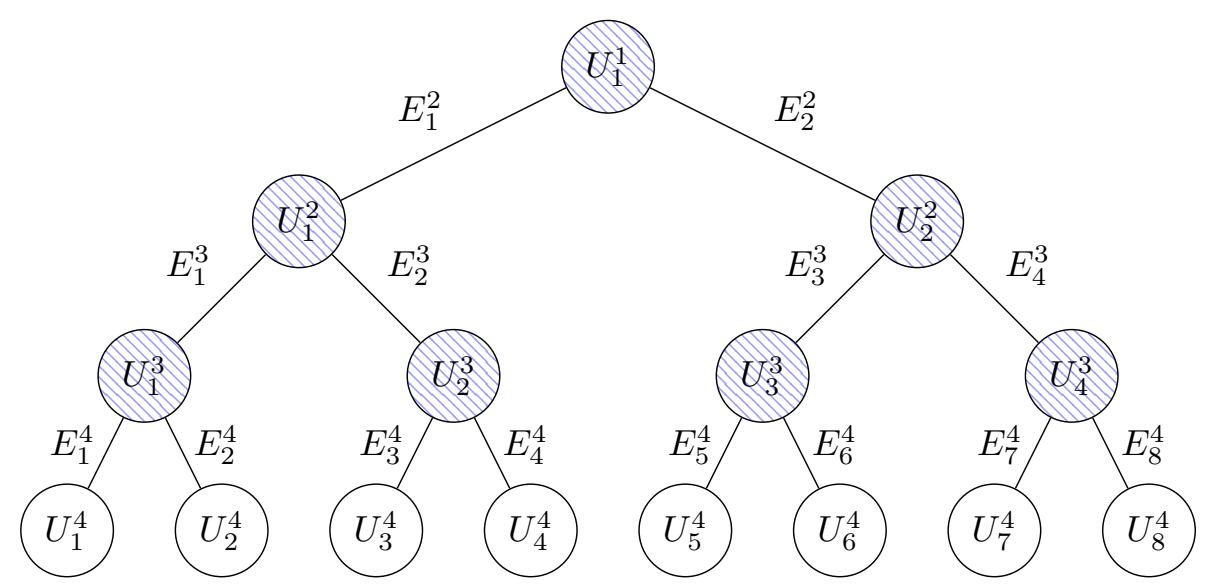

Figure 1.2: Basis tree $\mathcal{U}$ of an $\mathcal{H}^{2}$-matrix. Leaf nodes are stored explicitly, whereas inner nodes are represented implicitly using the transfer matrices $E$.

- A block column $A_{* s}^{l}$ is the set of leaves within a level $l$ that are defined by the column indexes of cluster $s$.

- A row basis $U_{t}^{l}$ is a common basis for the columns of the block row $A_{t *}^{l}$, organized in a row basis tree $\mathcal{U}$ defined by the cluster tree $T_{\mathcal{I}}$.

- A column basis $V_{s}^{l}$ is a common basis for the rows of the block column $A_{* s}^{l}$, organized in a column basis tree $\mathcal{V}$ defined by the cluster tree $T_{\mathcal{J}}$.

- A low rank matrix in this format is then stored as $A_{t s}^{l}=U_{t}^{l} S_{t s}^{l} V_{s}^{l}$, where $S_{t s}^{l}$ is a coupling matrix associated with the block, containing the interaction between the two clusters. These coupling matrices are organized in a matrix tree $\mathcal{S}$ defined by $T_{\mathcal{I} \times \mathcal{J}}$.

To eliminate the logarithm factor in the complexity estimates, an additional hierarchy is introduced in the row and column basis trees, called the nested basis property, wherein a basis node is expressed in terms of its children. Thus basis nodes are stored explicitly only at the leaves of the tree, and inner nodes can be computed from their children using small $k^{l} \times k^{l-1}$ interlevel transfer matrices. For example, one can computer an inner node $U_{i}^{l-1}$ from its two children, $U_{i_{1}}^{l}$ and $U_{i_{2}}^{l}$, and their corresponding 


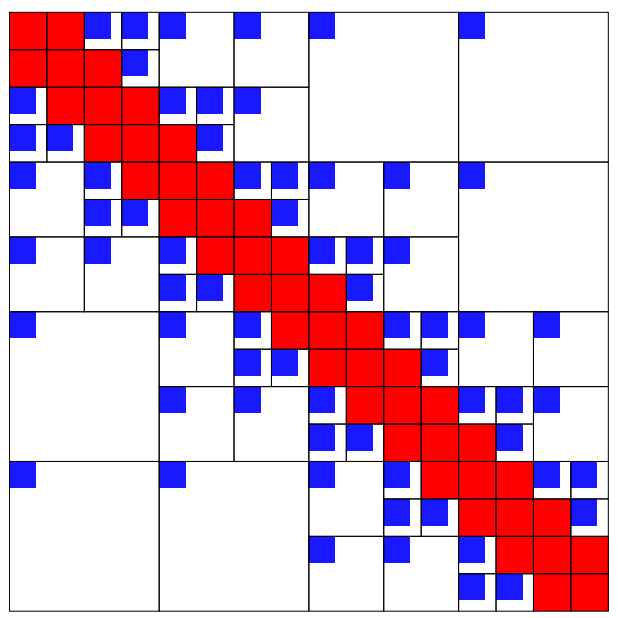

Figure 1.3: Leaves of matrix tree for a simple hierarchical matrix. Red blocks represent dense leaves and blue blocks are coupling matrices.

transfer matrices $E_{i_{1}}^{l}$ and $E_{i_{2}}^{l}$ as:

$$
U_{i}^{l-1}=\left[\begin{array}{cc}
U_{i_{1}}^{l} & \\
& \\
& U_{i_{2}}^{l}
\end{array}\right]\left[\begin{array}{l}
E_{i_{1}}^{l} \\
E_{i_{2}}^{l}
\end{array}\right] .
$$

Putting everything together, the $\mathcal{H}^{2}$-matrix representation consists of:

- The set of dense $m \times m$ leaves of the $\mathcal{H}$-matrix representation, shown as the red blocks of Figure 1.3 .

- A matrix tree $\mathcal{S}$ of $k^{l} \times k^{l}$ coupling matrices, whose leaves are shown as the blue blocks of Figure 1.3 .

- Row and column row basis trees $\mathcal{U}$ and $\mathcal{V}$ consisting of $m \times k$ explicitly stored basis leaves and implicitly represented inner nodes using the $k^{l} \times k^{l-1}$ interlevel transfer matrices $E$ and $F$ respectively. Figure 1.2 shows an example of the nested basis $\mathcal{U}$, with the implicitly represented inner nodes shaded. 


\subsection{Related Work}

The vast majority of work on general hierarchical matrices has been developed on the CPU, with multiple efficient implementations available [11] 12] [13] that have been used in a variety of applications. These libraries provide a comprehensive set of hierarchical matrix operations, such as construction of the hierarchical structures, discretization to generate matrix entries, arithmetic algorithms to perform approximative linear algebra and conversion routines that turn sparse or dense matrices into a hierarchical matrix format. A task-based parallel implementation of the hierarchical matrix LU factorization was used for efficient scheduling and parallel execution on the Intel Xeon Phi in [14].

Restricting the structure of the hierarchical matrix has allowed the development of fast solvers at the cost of increased memory storage due to larger ranks in the resulting low rank blocks. For example, fast factorization algorithms for hierarchically semiseparable (HSS) matrices, where the upper and lower triangular parts of the matrix are each part of a low rank matrix, have been developed in [15] and [16]. Hierarchically block-separable (HBS) matrices have a similar structure, where off-diagonal blocks are of low rank that grow geometrically in size with their distance from the main diagonal, but emphasize the telescoping nature of the matrix factorization [17] to use in the construction of direct solvers for integral equations [18].

Simplifying the structure even further, block low rank (BLR) or tile low rank (TLR) matrices partition the matrix into uniform sized blocks that are approximated as low rank matrices with varying bands of the diagonal kept in the original dense form. While not optimal in storage or runtime, the simplicity and flexibility of the format allows for significant speedups for multifrontal solvers [19][20] within the MUMPS software package [21] [22]. The TLR format enabled speedups of more than an order

of magnitude for large scale Cholesky factorization on matrix dimensions up to 11 million using dynamic runtime systems [23]. 
In many applications of interest where only fast matrix vector products are available, construction of hierarchical matrices has typically adopted either top-down strategies [24, 25], where the off-diagonal blocks of a level of the matrix are approximated using random structured vector sampling of the matrix with the upper levels peeled off with, or bottom-up approaches [26, 27], where low rank interpolative decompositions of blocks are generated from the finest to the coarsest levels and require arbitrary entries of the matrix to be available. The first strategy typically uses a fixed structure for the matrix, which limits its flexibility and potentially leads to matrices with large ranks, while the need for having explicit values for entries in the second strategy may limit its applicability. Shared and distributed memory hierarchical compression of SPD matrices into a weak-admissibility format are described in [28, 29].

While development of hierarchical methods has flourished on the CPU, there have been only limited efforts in the development of algorithms appropriate for GPU environments. For example, a recent work accelerated some of the readily vectorizable portions of the computation, such as setting up an initial stiffness matrix [30]. The $\mathcal{H}$-matrix vector multiplication has been parallelized on the GPU using parallel work queues [31] and on multiple GPUs [32] using optimized batched matrix vector kernels [33. However, methods addressing the core $\mathcal{H}^{2}$-matrix operations on GPUs are not yet available.

\subsection{Thesis Outline}

In this dissertation, I provide a set of hierarchical basic linear algebra subroutines (HBLAS) optimized for GPUs that can be used as a software layer for hierarchical solvers and preconditioners or as building blocks for other hierarchical operations. These operations can be thought of as generalizations of the linear algebra operations on dense matrices. Chapter 2 discusses the architectural challenges facing a GPU 
implementation of these operations and my approach to tackling them by splitting the computation into two phases. First, a marshaling phase generates batch kernel data from a flattened tree representation and then the linear algebra is executed using batched GPU kernels, the most complex of which are detailed in Chapter 3. Chapters 4. 5 and 6 discuss the details of the following five hierarchical matrix operations:

- hgemv: The product of a hierarchical matrix with one or more vectors generalizing the dense matrix vector product. A GPU implementation of this algorithm that achieves about $80 \%$ of the theoretical peak bandwidth of a P100 GPU is described in Chapter 4.

- horthog: The orthogonalization of the basis tree of the hierarchical matrix followed by a projection of its coupling matrices into the new basis. This operation can be thought of as a generalization of the dense QR decomposition, where an orthogonal basis is generated for the columns of the matrix, which is then projected into that basis. Chapter 5 describes the implementation details of this operation which achieves more than 2 TFLOP/s on a P100 GPU.

- hcompress: The (re)compression of the data in the hierarchical matrix to reduce its memory footprint by controlling the accuracy of the approximation. This generalizes the singular value decomposition (SVD), where a dense matrix can be approximated by truncating singular values below a certain threshold. Chapter 5 describes this operation which achieves more than $600 \mathrm{GFLOP} / \mathrm{s}$ on a P100 GPU in compressing a matrix for a problem size of 1 million in under 1.7 seconds.

- hlru: The low rank update of a hierarchical matrix, which generalizes the global low rank updates of a dense matrix, allowing local low rank updates to be made to individual nodes in the matrix tree. Chapter 6 describes this algorithm. 
- hara: Building on top of the previous operations, the hierarchical adaptive randomized approximation (HARA) allows the construction of a hierarchical matrix approximation of a matrix $A$ using only matrix vector products of random vectors with $A$. This is a generalization of the adaptive randomized approximation (ARA) used to generate approximations of dense matrices and is detailed in Chapter 6.

The hara operation enables the construction of several common matrix expressions, such as the product of two matrices or the iterates of an iterative approximate inversion scheme as hierarchical matrices, as shown in Chapter 7. A distributed parallel implementation of the hgemv operation is developed in Chapter 8, where I show that the methods can scale up to multiple GPUs efficiently. To demonstrate the effectiveness of the hierarchical operations, I develop applications for Hessians arising in inverse problems governed by PDEs in Chapter 9 and discrete operators for space-fractional diffusion equations in Chapter 10. In the former, the formally dense Hessian is constructed as a hierarchical matrix using up to ten times fewer matrix vector products than a global low rank approximation. In the latter, the quadratic computational and storage complexity of the discrete operators is reduced from their dense counterparts to the optimal linear complexity of the hierarchical approximation without sacrificing the accuracy of the solution. Finally, I list the key contributions and future directions of this work in Chapter 11. 


\section{Chapter 2}

\section{Efficient Hierarchical Matrix Operations on GPUs}

Modern scientific workstations generally feature manycore GPU accelerators, and algorithms that do not effectively take advantage of these architectures are unlikely to be competitive for scientific and financial applications. Modern GPU architectures feature decreasing ratios of memory bandwidth to processing power, smaller amounts of fast memory per processing core, and substantial latencies for accessing data in deep memory [34]. Competitive algorithms must therefore be able to orchestrate their computations for effective execution in this environment. One major benefit of the $\mathcal{H}^{2}$-matrix approximations is that their small, and asymptotically optimal, storage requirements make them amenable to effective GPU implementations. Through the efficient compression of discretized operators via their $\mathcal{H}^{2}$ representation, matrix data can be made to reside high on the memory hierarchy to allow substantial efficiencies to be realized, beyond the reduction in operations count that the representation produces.

The naturally recursive data structures and formulations of the hierarchical matrix algorithms, as well as the very small size of the matrices involved in the underlying linear algebra operations, do not however readily map to the throughput-oriented architecture of GPUs. In this chapter, I discuss some of their properties and architectural challenges that stand in the way of an efficient implementation and my approach to overcoming them. 


\subsection{GPU Considerations}

\subsubsection{GPU Kernels}

GPU kernels are launched by specifying a grid configuration that organizes threads into blocks and blocks into a grid. Launching a GPU kernel causes a short stall (as much as 10 microseconds) as the kernel is prepared for execution. Figure 2.1 shows an example from a visual profiler where the kernel launch overhead (shown in orange in the timeline) greatly exceeds the execution duration for the kernel (shown in blue in the lower right corner). This kernel launch overhead prevents kernels that complete their work faster than the overhead from executing in parallel, essentially serializing them. To overcome this limitation when processing small workloads, the work is batched into a single kernel call when possible [35, 36]. All operations can then be executed in parallel without incurring the kernel launch overhead, with the grid configuration used to determine thread work assignment.

\subsubsection{Warps}

A warp is a group of threads (32 threads in current generation GPUs, such as the NVIDIA P100) within a block that executes a single instruction in lockstep, without requiring any explicit synchronization. The occupancy of a kernel is the ratio of active warps to the maximum number of warps that a multiprocessor can host. This metric is dependent on the amount of resources that a kernel uses, such as register and shared

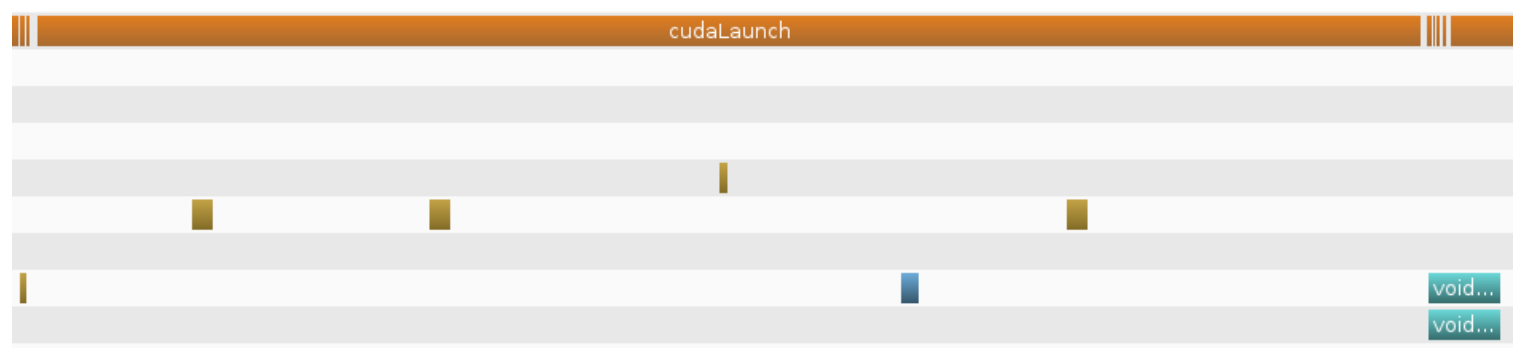

Figure 2.1: Kernel launch overhead shown on the execution timeline in orange for a kernel in blue that does very little work. 


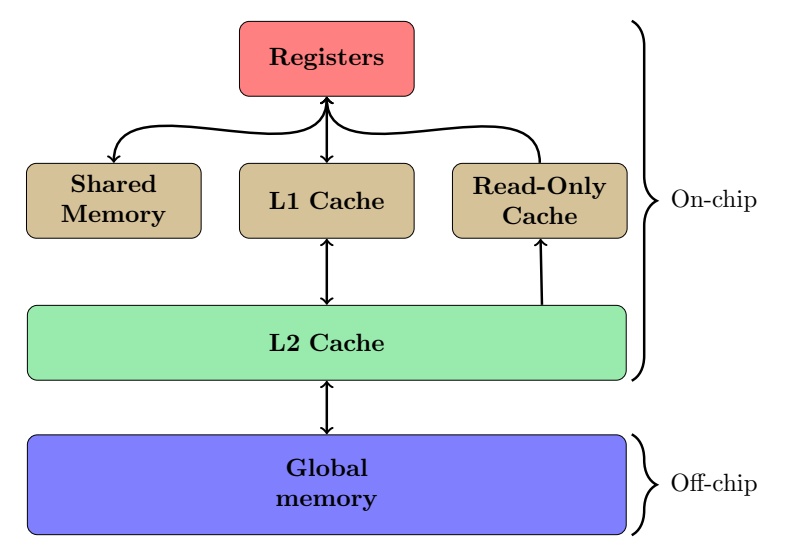

Figure 2.2: The memory hierarchy of a modern GPU.

memory usage and kernel launch configuration, as well as the compute capability of the card ([37] for more details). While not a requirement for good performance [38], it is generally a good idea to aim for high occupancy.

\subsubsection{Memory}

Memory on the GPU is organized into a hierarchy of memory spaces as shown in Figure 2.2. At the bottom, global memory is accessible by all threads and is the most plentiful but the slowest memory. The next space of interest is the shared memory which is accessible only by threads within the same block and is configurable with the L1 cache to be at most $48 \mathrm{~KB}$ per thread block on current generation GPUs. Shared memory is very fast and acts as a programmer controllable cache. Finally, registers, which are local to the threads, are the fastest of all memory, but the total number of registers usable by a thread without performance implications is limited. If a kernel needs more registers than the limit, then registers are spilled to "local" memory, which is in the slow but cached global memory. Making good use of the faster memories and avoiding excessive accesses to the slower ones is key to good performance on the GPU. As such, it is common to use blocking techniques in many algorithms, where a block of data is brought in from global memory and processed in one of the faster memories. 


\subsection{Architectural Challenges}

All matrix blocks within the $\mathcal{H}^{2}$-matrix representation as described in Section 1.1.2 are quite small, with block sizes dependent on the ranks of the low rank blocks and the size of the leaf clusters in the cluster trees. Attempting to execute the constituent linear algebra operations of the overall hierarchical matrix operations on a GPU leads to very poor performance, because a GPU is a throughput-oriented architecture that requires massive levels of parallelism to hide their relatively high latency. This stands in contrast to the latency-oriented architecture typically used in CPUs. A naive recursive implementation that calls GPU routines from the CPU will perform quite poorly. The same applies to implementations that rely on the dynamic parallelism feature of GPUs that allow GPU routines to be called from the GPU itself, since those need execution units that perform relatively large amounts of work. Standard task schedulers that determine parallel work on the CPU typically perform poorly as well, since the individual tasks will be bound by the kernel launch overheads even when launched in parallel on the CPU. I overcome these challenges in two ways:

- A flattened tree data structure presented in Section 2.3 is used for both the basis and matrix trees, allowing the implementation of fast static scheduling of all operations performed on a level $l$ of a tree in a phase called "marshaling". Marshaling prepares for the execution of the entire set of operations on level $l$ by efficiently gathering data from the tree in a single GPU routine call that will then be executed using GPU batched routines.

- A large set of efficient GPU batched routines are developed to support the execution of the various marshaled operations using a single GPU call, including batched SVD, QR factorization, adaptive randomized approximation, matrix vector products, random matrix block generation, and more. Since the overall performance of a hierarchical operation is highly dependent on the performance 
of these batched operations, the details of some of the more complex ones are discussed in Chapter 3 .

By separating the computation into two phases, marshaling and batched execution, the maintainability and portability of the software is also greatly increased, since the marshaling is built on top of standard parallel programming constructs, such as parallel for loops, and the batched routines can be easily implemented on architectures other than the GPU. For example, a batched routine on the CPU is simply a parallel for loop around standard BLAS and LAPACK routines. This allows the methods developed here to run on both the CPU and GPU using the same code base with simple abstractions around the batched routine execution for each architecture. Marshaling also enables a clean separation of concern between tree operations and the execution of the linear algebra operations that are at the heart of each hierarchical algorithm. This makes the resulting code easier to maintain and future proofs it to benefit from independent advances in batched linear algebra. The marshaling routines are specific to the type of hierarchical operation being executed. Many examples are given in Chapters 4, 5, and 6.

\subsection{Flattening the Trees for Efficient GPU Marshaling}

While a recursive data structure is a natural representation of the trees that make up a hierarchical matrix, marshaling the operations on a recursive data structure using dynamic parallelism on the GPU adds significant overhead and complexity to each operation, while attempting to marshal on the CPU will also require relatively slow copying of the marshaled data to the GPU. To efficiently marshal on the GPU, I flatten the trees by levels, store the level data in contiguous chunks of memory, and implement marshaling for each operation using specialized kernels that process each level in parallel to produce the necessary data for the batch kernels. Since every kernel

call uses a single launch configuration, the operations handled by each batch routine 


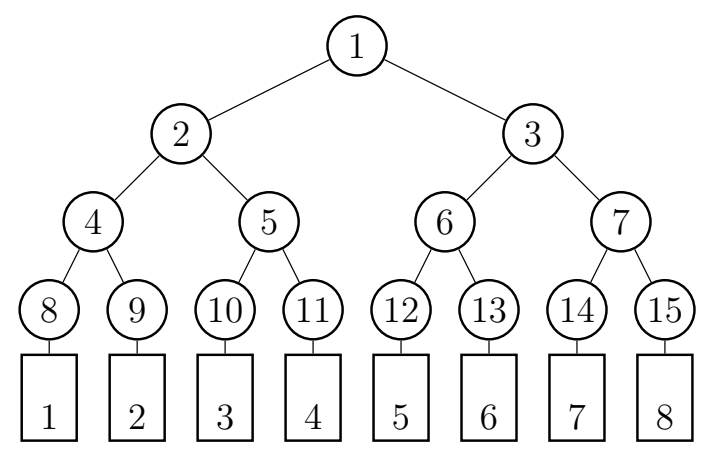

\begin{tabular}{|c|c|c|c|c|c|c|c|c|c|c|c|c|c|c|c|}
\hline Level Pointers & 1 & 2 & 4 & 8 & 16 & & & & & & & & & & \\
\hline parent & - & 1 & 1 & 2 & 2 & 3 & 3 & 4 & 4 & 5 & 5 & 6 & 6 & 7 & 7 \\
\hline head & 2 & 4 & 6 & 8 & 10 & 12 & 14 & - & - & - & - & - & - & - & - \\
\hline next & - & 3 & - & 5 & - & 7 & - & 9 & - & 11 & - & 13 & - & 15 & - \\
\hline
\end{tabular}

Figure 2.3: Basis tree structure. Any data associated with the basis tree uses the node numbers to locate the position of the node data in memory.

must have the same size; in the $\mathcal{H}^{2}$-matrix setting, this translates to a fixed rank per level of the trees. More robust batch kernels that permit non-uniformly-sized batch items will be needed to overcome this limitation. This will be the focus of future work.

The flattened structure of the tree is represented by three arrays: head, next, and parent. The head array contains the node index of the first child of a node while each entry in the next array gives the index of the next sibling of a node. The parent array contains the parent node index of each node, allowing tree traversals in any direction. The level pointers array store the limits of the node indexes within the three arrays for each level. An example of this storage scheme for the structure of the basis tree is depicted in Figure 2.3 . 


\section{Chapter 3}

\section{Batched Linear Algebra Subroutines}

The performance of hierarchical matrix operations is almost entirely dependent on the efficiency of the supporting batched linear algebra subroutines (conveniently overloading the BLAS acronym). In this chapter, I discuss the GPU implementation of some of the more complex kernels, including batched QR decomposition, the singular value decomposition of small matrices, and randomized algorithms for computing low rank approximations of matrices, which are used during the orthogonalization and compression of the basis trees in Chapter 5 .

\subsection{Background}

\subsubsection{QR Factorization}

The QR factorization decomposes an $m \times n$ matrix $A$ into the product of an orthogonal $m \times m$ matrix $Q$ and an upper triangular $m \times n$ matrix $R$ [39]. One can also compute a reduced form of the decomposition where $Q$ is an $m \times n$ matrix and $R$ is $n \times n$ upper triangular. The most common $\mathrm{QR}$ algorithm is based on transforming $A$ into an upper triangular matrix using a series of orthogonal transformations generated using Householder reflectors. Other algorithms such as the Gram-Schmidt or Modified Gram-Schmidt can produce the QR factorization by orthogonalizing a column with all previous columns; however, these methods are less stable than the Householder orthogonalization and the orthogonality of the resulting $Q$ factor suffers with the condition number of the matrix. Another method is based on Givens rotations, where 


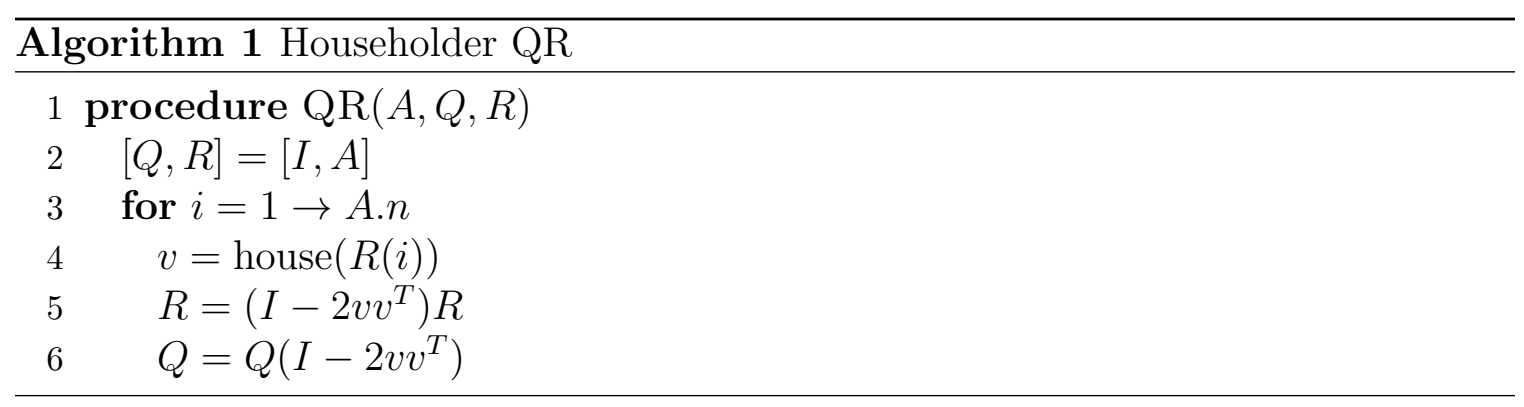

entries in the subdiagonal part of the matrix are zeroed out to form the triangular factor and the rotations are accumulated to form the orthogonal factor. This method is very stable and has more parallelism than the Householder method; however it is more expensive, doing about 50\% more work, and it is more challenging to extract the parallelism efficiently on the GPU. For my implementation, I rely on the Householder method due to its numerical stability and simplicity. The method is described in pseudo-code in Algorithm 1 .

\subsubsection{SVD Algorithms}

The singular value decomposition (SVD) is a factorization of a general $m \times n$ matrix $A$ of the form

$$
A=U \Sigma V^{*}
$$

$U$ is an $m \times m$ orthonormal matrix whose columns $U_{i}$ are called the left singular vectors. $\Sigma$ is an $m \times n$ diagonal matrix whose diagonal entries $\sigma_{i}$ are called the singular values and are sorted in decreasing order. $V$ is an $n \times n$ orthonormal matrix whose columns $V_{i}$ are called the right singular vectors. When $m>n$, one can compute a reduced form $A=\hat{U} \hat{\Sigma} V^{*}$ where $\hat{U}$ is an $m \times n$ matrix and $\hat{\Sigma}$ is an $n \times n$ diagonal matrix. One can easily obtain the full form from the reduced one by extending $\hat{U}$ with $(m-n)$ orthogonal vectors and $\hat{\Sigma}$ with an $(m-n)$ zero block row. Without any loss of generality, I will focus on the reduced SVD of real matrices.

Most implementations of the SVD are based on the two-phase approach popu- 


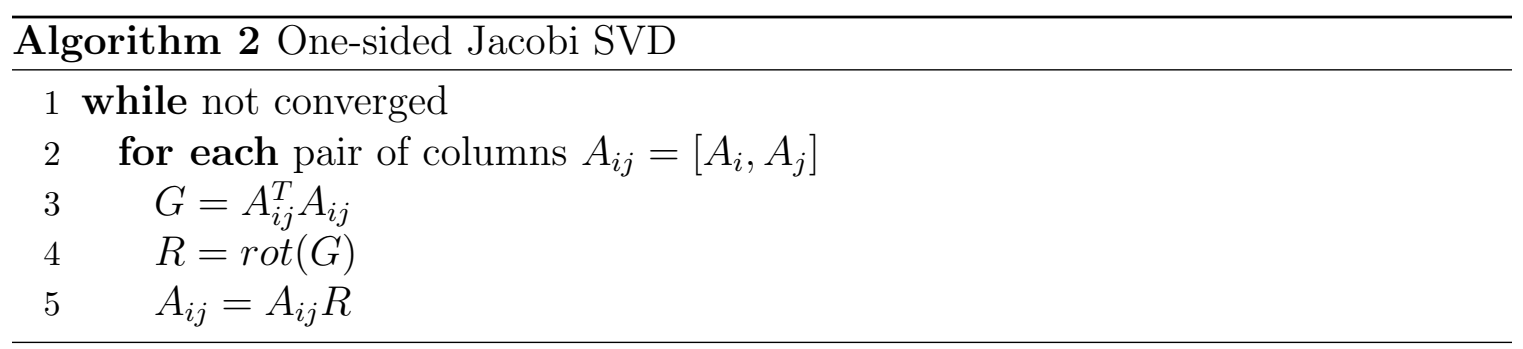

larized by Trefethen et al. [40], where the matrix $A$ first undergoes bidiagonalization of the form $A=Q_{U} B Q_{V}^{T}$, where $Q_{U}$ and $Q_{V}$ are orthonormal matrices and $B$ is a bidiagonal matrix. The matrix $B$ is then diagonalized using some variant of the QR algorithm, the divide and conquer method or a combination of both to produce a decomposition $B=U_{B} \Sigma V_{B}^{T}$. The complete SVD is then determined as $A=\left(Q_{U} U_{B}\right) \Sigma\left(Q_{V} V_{B}\right)^{T}$ during the backward transformation. These methods require significant algorithmic and programming effort to become robust and efficient while still suffering from a loss of relative accuracy [41].

An alternative is the one-sided Jacobi method where all $n(n-1) / 2$ pairs of columns are repeatedly orthogonalized in sweeps using plane rotations until all columns are mutually orthogonal. When the process converges (i.e., all columns are mutually orthogonal up to machine precision), the left singular vectors are the normalized columns of the modified matrix with the singular values as the norms of those columns. The right singular vectors can be computed either by accumulating the rotations or by solving a system of equations. The motivating applications do not need the right vectors, so I omit the details of computing them. Algorithm 2 describes the one-sided Jacobi method. Since each pair of columns can be orthogonalized independently, the method is also easily parallelized. The simplicity and inherent parallelism of the method make it an attractive first choice for an implementation on the GPU. 


\subsubsection{Adaptive Randomized Approximation}

An $m \times n$ matrix $M$ is said to have low numerical rank when it can be well approximated by a low rank matrix of the form $M \approx A B^{T}$, where $A$ and $B$ are $m \times k$ and $n \times k$ matrices respectively and $k$ is called the numerical rank. The numerical rank is typically much smaller than $m$ and $n$, allowing computations with and storage of $M$ to be more efficient as long as $k \leq \frac{m n}{m+n}$. Randomized methods have been developed [42] to compute the low rank approximation using only matrix vector multiplication, making it an excellent choice for black box routines where matrix entries may not be available. While these methods have been used primarily for very large problems, I develop batched GPU routines for these algorithms that show substantial speedups when compared with the SVD for computing low rank approximations of large batches of small matrix blocks entirely on the GPU. In this section, I will discuss some details of the algorithms and their implementation on the GPU.

\section{Fixed rank}

When the rank $k$ of the matrix is known beforehand, the fixed rank randomized approximation method, described in Algorithm 3, can be used to compute the low rank approximation of an $m \times n$ matrix $M$. An approximate orthogonal basis $Q$ for the column space of $M$ is first computed as the left factor of the low rank approximation. This involves producing a random $m \times k$ sample matrix $Y$ from the range of $M$ by multiplying the matrix by a random $n \times k$ matrix $\Omega$. The sample matrix can then be orthogonalized by performing a standard QR decomposition and discarding the triangular factor. It is worth noting that for matrices whose singular values decay rapidly, this method is sufficient to produce a good approximate basis $Q$; however, when the singular values decay slowly, further refinement of the basis by subspace iteration is required [42]. This enhancement to the algorithm is not discussed here since the matrices I target fall in the former category. Once the approximate orthogo- 


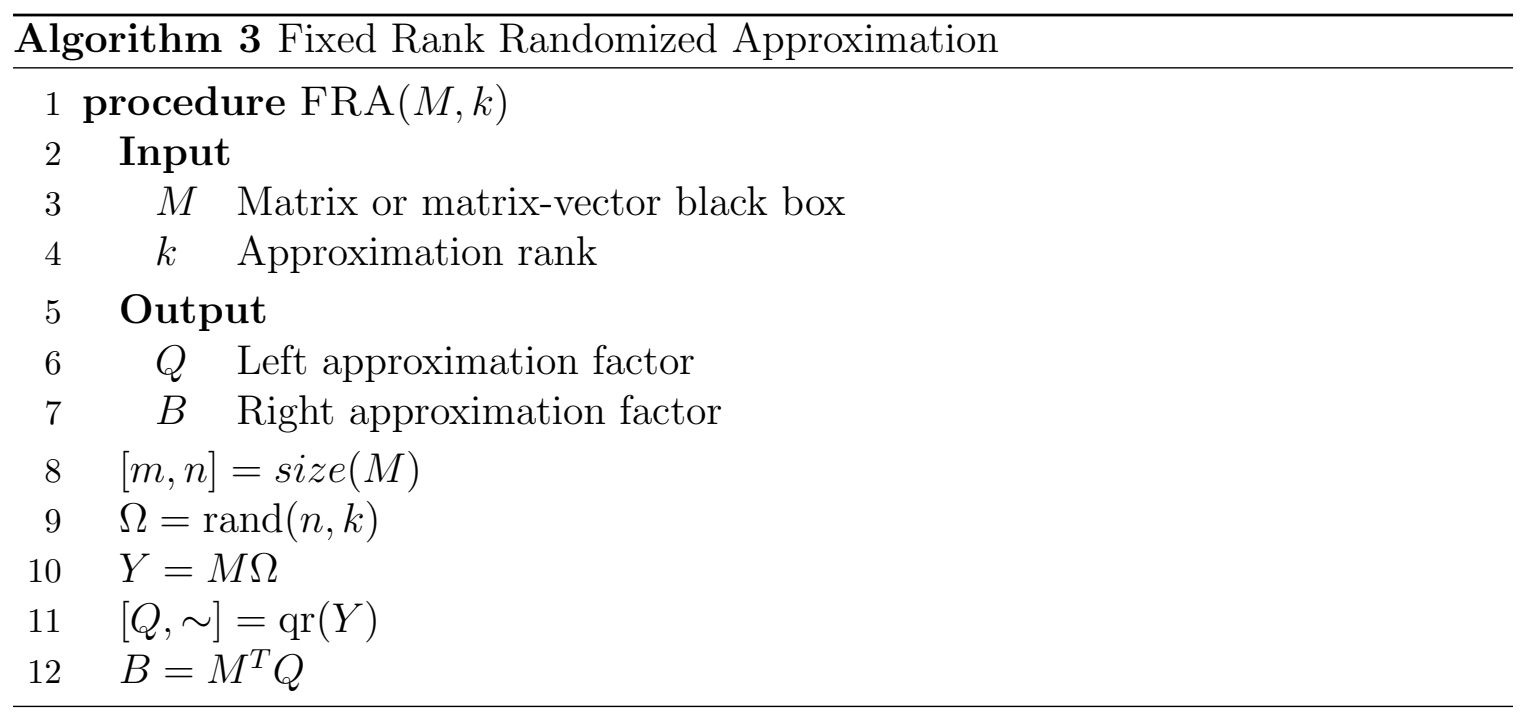

nal basis $Q$ has been determined, the right factor $B$ of the low rank approximation of $M \approx Q B^{T}$ can be computed simply by forming the product $B=M^{T} Q$. If additional rank reduction is required (to satisfy some fixed error threshold), an approximate singular value decomposition can be easily obtained from this low rank form by computing the QR decomposition of $B$ and then the SVD of the small $k \times k$ triangular factor. This method has been implemented as a batched GPU routine and used to accelerate the compression of hierarchical matrices [43] in place of the full singular value decomposition.

\section{Adaptive rank}

The rank of a matrix that satisfies a fixed precision is not typically known beforehand, making it difficult to use the fixed rank approximation in applications that have specific error tolerance requirements. Based on the adaptive randomized range finder algorithm [42], a fixed precision variant of the randomized approximation that adaptively determines the needed number of samples is described in Algorithm 4 . It takes advantage of the fact that the basis can be constructed iteratively and the approximation error can be easily evaluated at each step. Instead of producing all the samples at once, the orthogonal basis is generated by repeatedly sampling the 


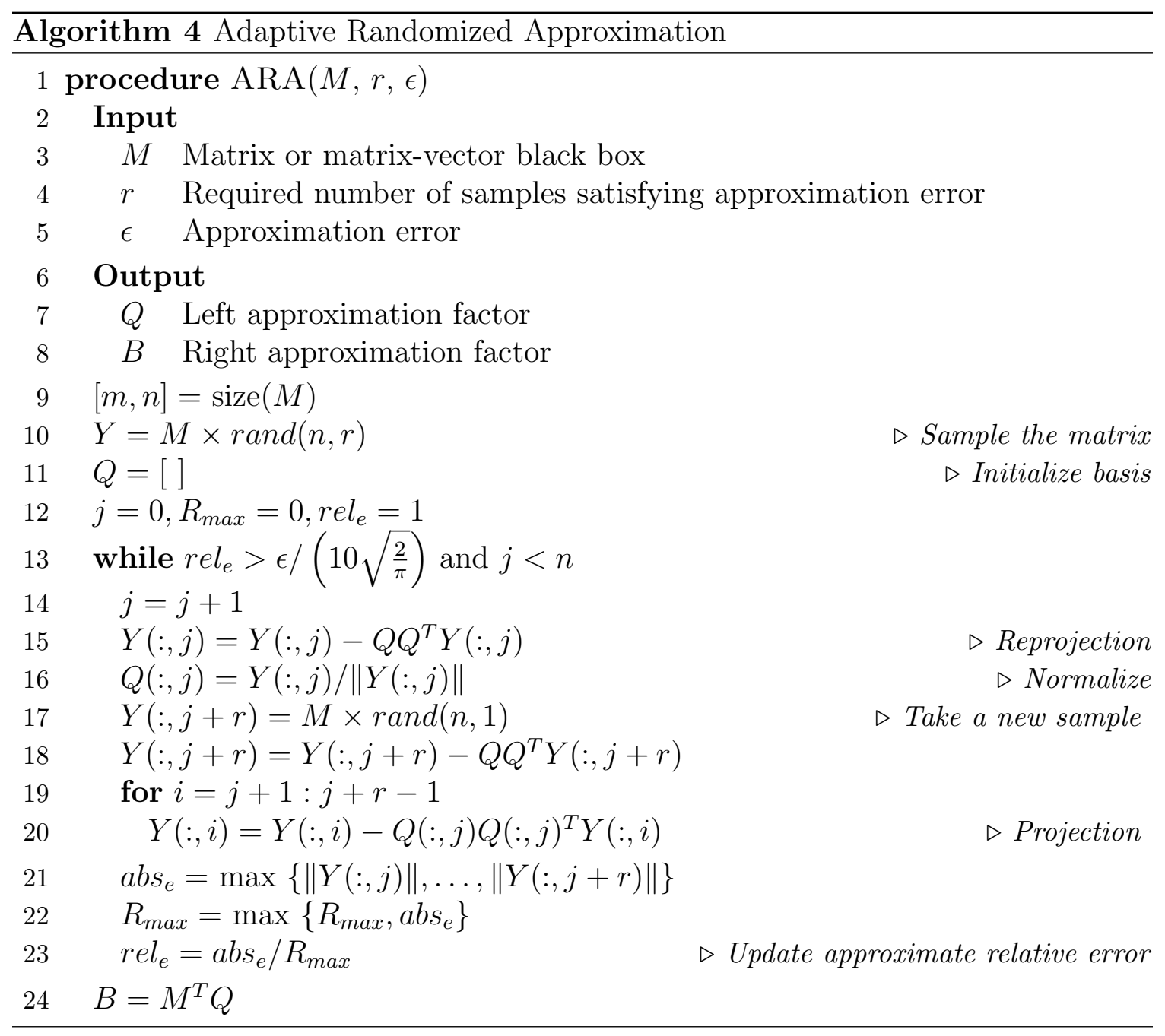

matrix and orthogonalizing using a process similar to Gram Schmidt (GS) with one reorthogonalization step [44, 45], until $r$ consecutive projected samples satisfy the error threshold. The absolute error is approximated by the maximum norm of the last $r$ sampled vectors while the relative error is approximated by keeping track of the maximum norm of all projected sampled vectors. The relative error approximation is not a standard one, but is easily obtained as a byproduct of the orthogonalization and performs well in practice. More complex methods, such as approximating the largest singular value, can be used to get a tighter relative error; however in practice the additional computational cost is not worth any potential reduction in the computed rank. 


\subsection{Batched QR Decomposition}

In this section, I discuss implementation details of my batched QR kernel and compare it with other implementations from the MAGMA 2.2 [46] and cuBLAS 8 [47] libraries.

\subsubsection{Implementation}

One benefit of the Householder algorithm is that the application of reflectors to the trailing matrix (line 5 of Algorithm 1) can be blocked together and expressed as a matrix-matrix multiplication (Level 3 BLAS) instead of multiple matrix-vector multiplications (Level 2 BLAS). The increased arithmetic intensity typically allows performance to improve when the trailing matrix is large. However, for small matrix blocks, the overhead of generating the blocked reflectors from their vector form as well as the lower performance of the matrix-matrix multiplication for small matrices hinders performance. One can obtain better performance by applying multiple reflectors in their vector form and performing the transpose matrix-vector multiplication efficiently within a thread block [48]. First, the regular factorization on a column block $P$ (called a panel) is carried out. The entire panel is stored in registers, with each thread storing one row of the panel, and the transpose matrix-vector product is computed using a series of reductions using shared memory and warp shuffles [49] which allow threads within a warp to read each other's registers. Figure 3.1 shows the data layout for a hypothetical warp of size 8 with 4 columns in registers and a warp reduction using shuffles. Once the panel is factored, the reflectors can be applied to the trailing sub-matrix in a separate kernel that is optimized for performing the core matrix-vector product in the update. In this second kernel, both the factored panel $P$ and a panel $M_{i}$ of the trailing sub-matrix $M$ are loaded to registers and the reflectors are applied one at a time, updating the trailing panel in registers. Taking an example of a $32 \times 8$ trailing panel $M_{i}$, I compute for each reflector the matrix-vector product $M_{i}^{T} v$ by flattening the $32 \times 8$ product into a reduction of a 256 vector in shared 
memory that has been padded to avoid bank conflicts. The reduction can then be serialized until it reaches a size of 32 , where a partial reduction to a vector of size 8 can take place in 2 steps. This final vector is the product $M_{i}^{T} v$ which can then be quickly applied to the registers storing $M_{i}$. This process is repeated for each trailing panel within the same kernel to maximize the use of the reflectors which have been stored in registers. Figure 3.2 shows one step of a panel factorization and the application of its reflectors to the trailing submatrix. Since threads are limited to 1024 per block on current architectures, I use the approach developed in [50] to factorize larger matrices. First, panels up to the thread block limit are factorized in a single kernel call. The panels below the first are then factorized by first loading the triangular factor into shared memory and then proceeding with the panel factorization as before, taking the triangular portion into consideration when computing reflectors and updates. To keep occupancy up for the small matrices on devices where the resident block limit could be reached before the thread limit, I assign multiple operations to a single thread block. For a batch of $N$ matrices of dimensions $m \times n$, kernels can be launched using $N / b$ thread blocks of size $m \times b$, where each thread block handles $b$ operations.

\subsubsection{Performance}

Figures $3.3 \mathrm{a}$ and $3.3 \mathrm{~b}$ show the performance of my batched QR for 1000 square and rectangular matrices with a panel width of 16, tuned for the P100 GPU. I compare against the vendor implementation in cuBLAS as well as the high performance library MAGMA. The proposed version performs markedly better for rectangular matrices with column size of 32 . As with many of the results in this chapter, GPU performance of the batched routine was stable and showed negligible scatter at each size. For the larger square matrix sizes where the blocked algorithm starts to show its performance benefits, MAGMA begins to catch up, but is still outperformed up to matrix dimen- 

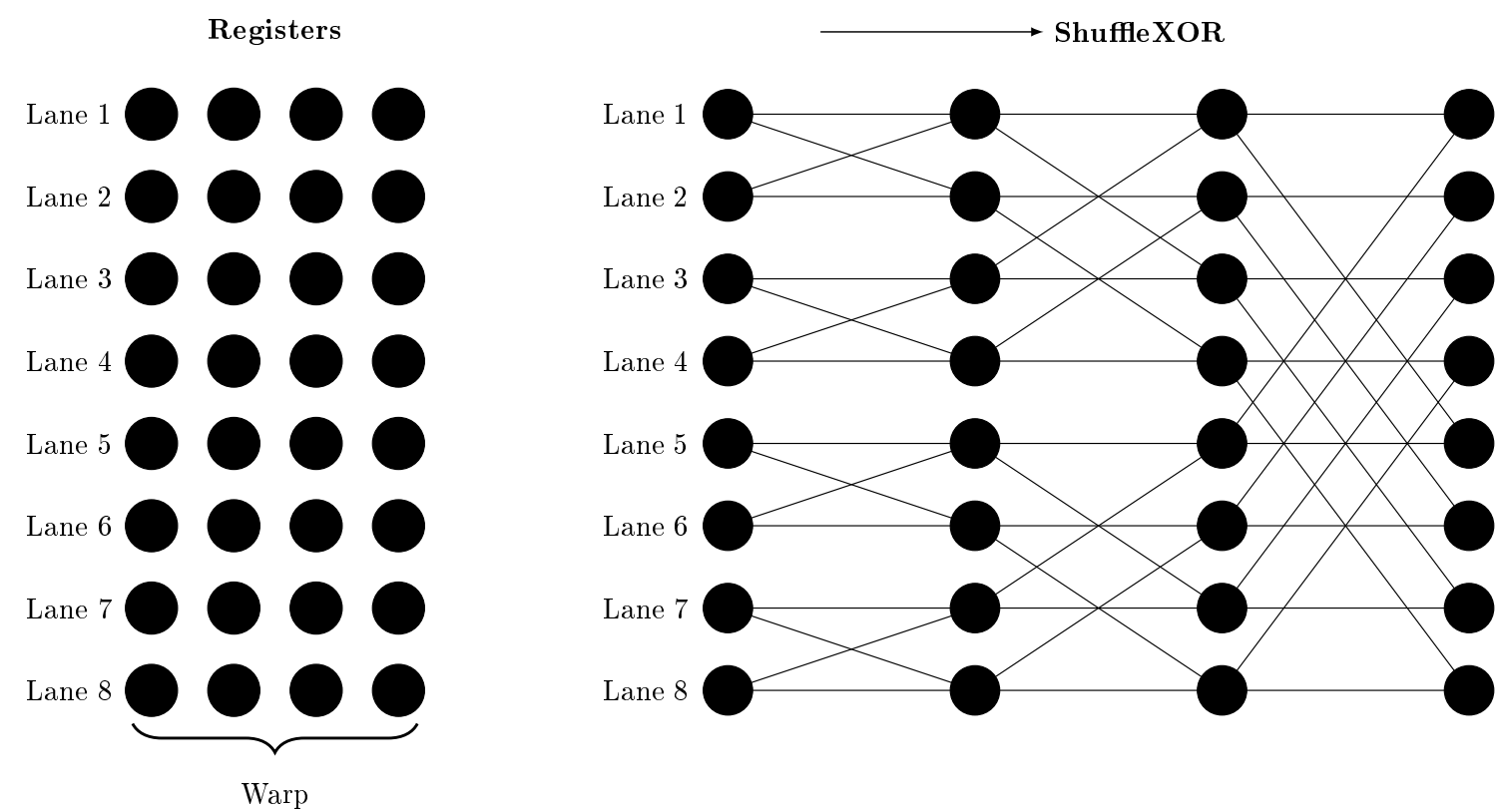

Figure 3.1: Left: matrix rows allocated to thread registers in a warp. Right: parallel warp reduction using shuffles within registers.
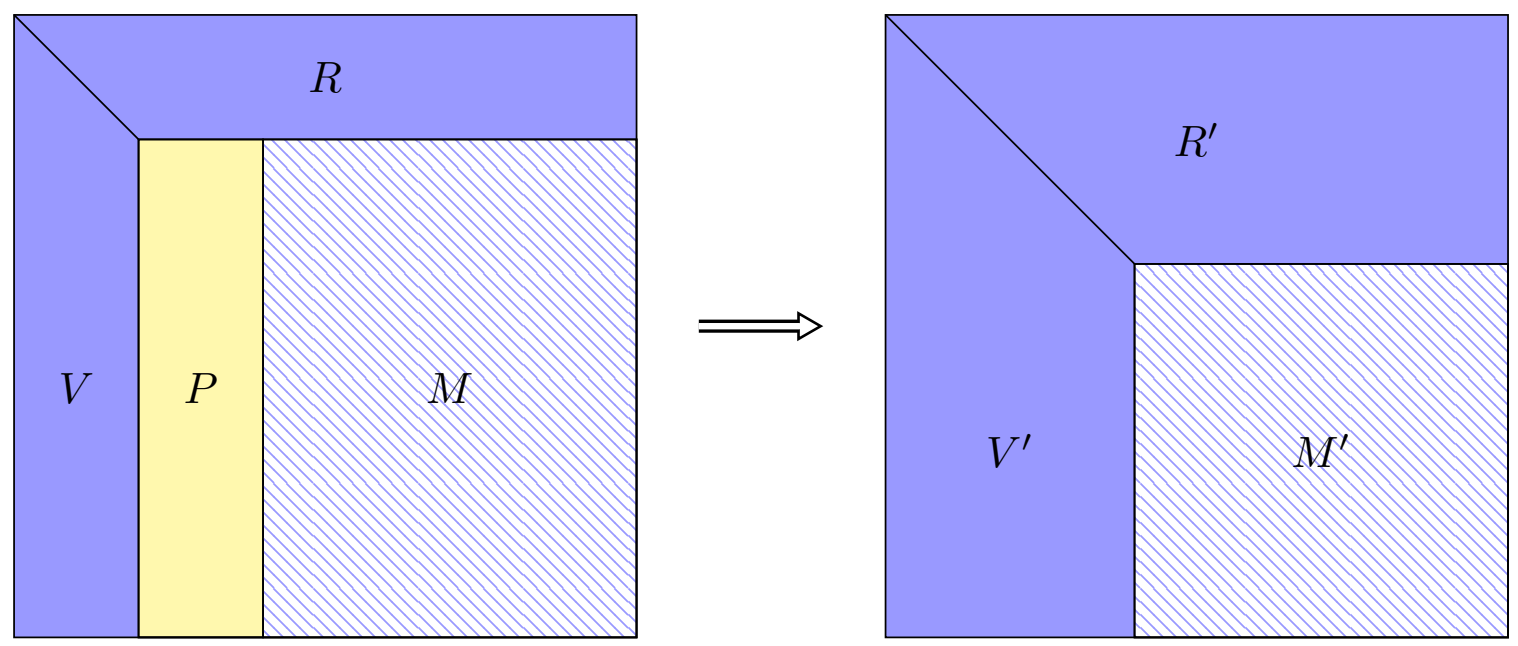

Figure 3.2: One step of the QR factorization where a panel $\mathrm{P}$ is factored to produce a triangular factor $\mathrm{R}$ and reflectors $\mathrm{V}$ which are used to update the trailing sub-matrix M. 


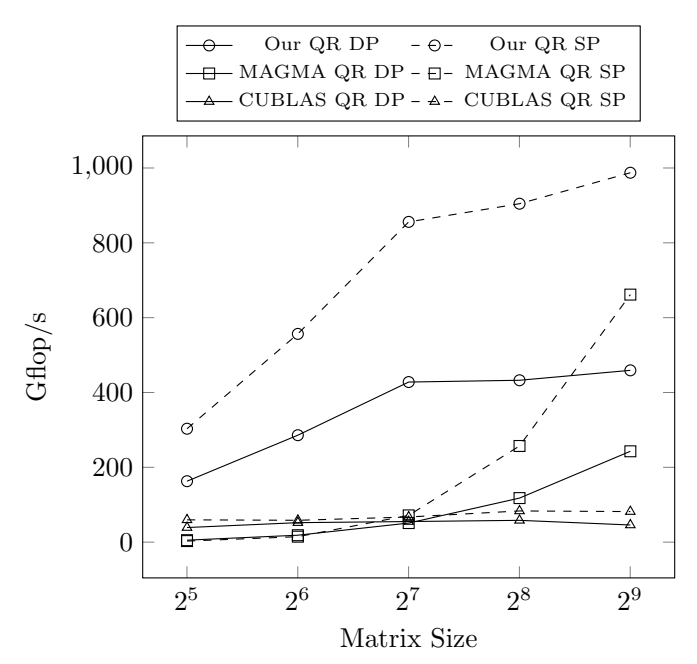

(a) Batched QR kernel performance for square matrices.

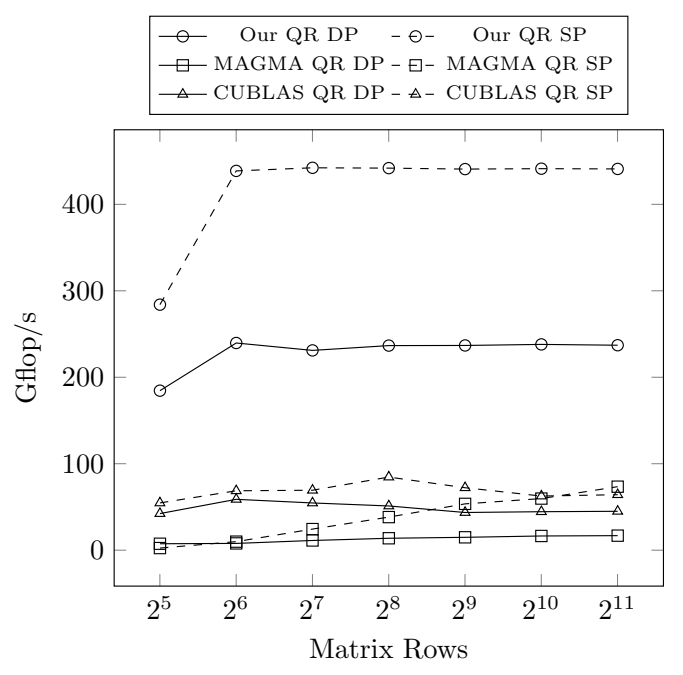

(b) Batched QR kernel performance for rectangular matrices with a fixed column size of 32 .

Figure 3.3: Comparing batched QR kernels for 1000 matrices of varying size on a P100 GPU in single and double precision.

sions of 512, as shown. A nested implementation where my kernel can be used to factor relatively large panels in a blocked algorithm will likely show some additional performance improvements for the large square matrices, but I leave that as future work.

\subsection{Register Memory One-Sided Jacobi}

In this section, I will discuss the first batched SVD kernel where the matrix data is hosted in registers and analyze the performance of the resulting kernel.

\subsubsection{Implementation}

In this implementation, to avoid repeated global memory accesses, I attempt to fit the matrix in register memory using the same layout as the panel in the QR factorization, i.e., one row per thread; however, the number of registers that a thread uses has an impact on occupancy which can potentially lead to lower performance. In addition, 
once the register count exceeds the limit set by the GPU's compute capability, the registers spill into "local" memory which resides in cached slow global memory. Since an entire matrix row is stored in the registers of one thread, I use the serial onesided Jacobi algorithm to compute the SVD where column pairs are processed by the threads one at a time. The bulk of the work lies in the computation of the Gram matrix $G=A_{i j}^{T} A_{i j}$ (line 3 of Algorithm 2 and in the update of the columns (line 5 ). Since the Gram matrix is symmetric, this boils down to three dot products which are executed as parallel reductions within the warp using warp shuffles. The computation of the $2 \times 2$ rotation matrix as well as the convergence test is performed redundantly in each thread. Finally, the column update is done in parallel by each thread on its own register data. As with the QR kernel, I keep occupancy up for the smaller matrix sizes by assigning multiple SVD operations to a single block of threads with each operation assigned to a warp to avoid unnecessary synchronizations.

\subsubsection{Performance}

I generate batches of 1000 test matrices with varying condition numbers using the latms LAPACK routine and calculate performance based on the total number of rotations needed for convergence. Figures $3.4 \mathrm{a}$ and $3.4 \mathrm{~b}$ show the performance on a P100 GPU of the register-based batched SVD kernel and the effect increased register usage has on occupancy. Profiling the kernel, the Gram matrix computation takes about 500 cycles, column rotations take about 240 cycles, and the redundantly computed convergence test and rotation matrices dominate at 1900 cycles. The fact that the redundant portion of the computation dominates means that it is preferable to assign as few threads as possible when processing column pairs. Due to the low occupancy for the larger matrix sizes and the register spills to local memory for matrices larger than 30 , it is obvious that the register approach will not suffice for larger matrix sizes. This leads to the next implementation based on the slower but more parallel-friendly 


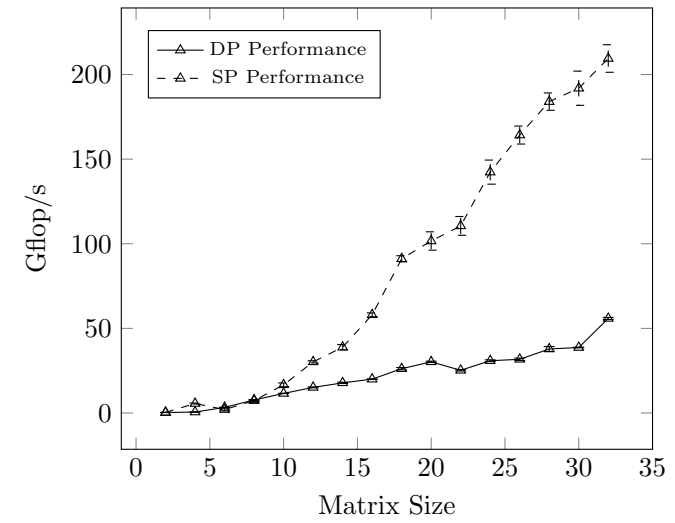

(a) Kernel performance in GFLOP/s and achieved occupancy.

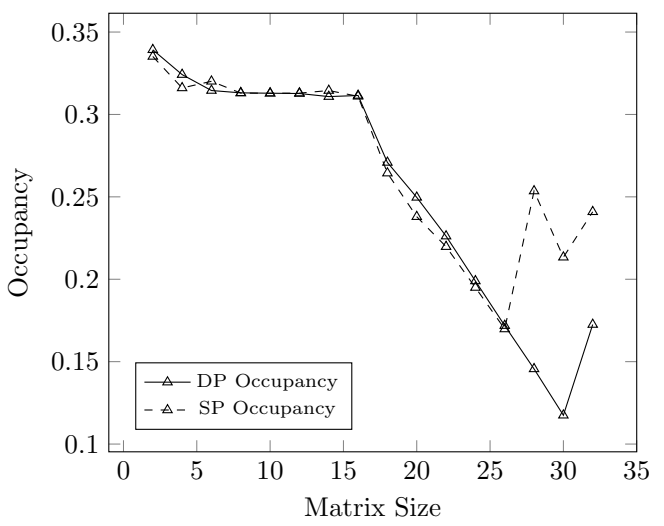

(b) The effect of increasing the matrix size on the occupancy of the register kernel.

Figure 3.4: Performance of the batched register memory SVD on a P100 GPU for 1000 matrices of varying size in single and double precision arithmetics.

shared memory.

\subsection{Shared Memory One-Sided Jacobi}

While the register-based SVD performs well for very small matrix sizes, a kernel that can handle larger sizes and maintain reasonably high occupancy is still needed. This leads to building a kernel based on shared memory, the next level of the GPU memory hierarchy. This section discusses the implementation details of this kernel and analyzes its performance when compared with the register kernel.

\subsubsection{Implementation}

In this version, the matrix is stored entirely in shared memory, which is limited to at most $48 \mathrm{~KB}$ per thread block on current generation GPUs. Using the same thread assignment as the register-based kernel would lead to very poor occupancy due to the high shared memory consumption, where potentially only a few warps will be active in a multiprocessor. Instead, I exploit the inherent parallelism of the one-sided Jacobi to assign a warp to a pair of columns, i.e., there are $n / 2$ warps processing 
an $m \times n$ matrix stored in shared memory. There are a total of $n(n-1) / 2$ pairs of columns, so I must generate all pairings in $n-1$ steps, with each step processing $n / 2$ pairs in parallel. There are many ways of generating these pairs, including round robin, odd-even, and ring ordering [51, 52]. I implement the round robin ordering using shared memory to keep track of the column indexes of the pairs with the first warp in the block responsible for updating the index list after each step. Figure 3.5 shows this ordering for a matrix with 8 columns. When the number of matrix rows exceeds the size of the warp, the thread-per-row assignment no longer allows the use of fast warp reductions, which would force the consumption of even more resources, as the reductions would now have to be done in shared memory. Instead, I assign multiple rows to a thread, serializing a portion of the reduction over those rows until warp reductions can be used. This follows the observation in Section 3.3 .2 to assign as few threads as possible to process column pairs, frees up valuable resources and increases the overall performance of the reduction. Row padding is used to keep the rows at multiples of the warp size, and column padding is used to keep the number of columns even. Kernels can then be launched using $32 \times n / 2$ threads to process each matrix. Figures $3.6 \mathrm{a}$ and $3.6 \mathrm{~b}$ show examples of the thread allocation and reductions for a $8 \times 8$ matrix using a hypothetical warp size of 4 .

\subsubsection{Performance}

Figures $3.7 \mathrm{a}$ and $3.7 \mathrm{~b}$ show the performance of the parallel shared SVD kernel compared to the serial register SVD kernel on a P100 GPU. One can see the improved growth in performance in the shared memory kernel due to the greater occupancy as well as the absence of any local memory transactions. Looking at the double precision occupancy, one notices two dips in occupancy at matrix sizes 22 and 32 as the number of resident blocks become limited by the registers/block limits of the device, dropping to 2 and then 1 resident blocks. Performance increases steadily from there 

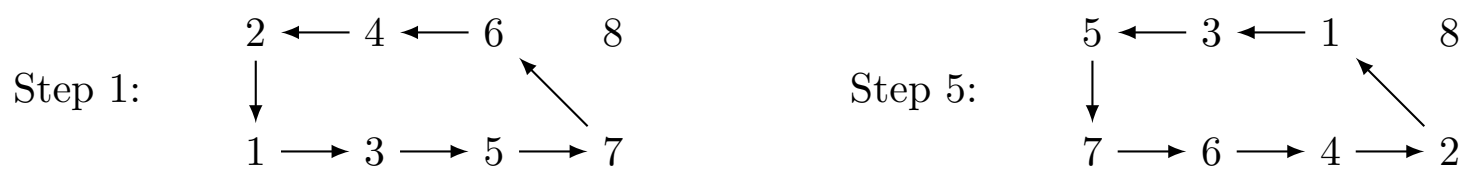

Step 2:
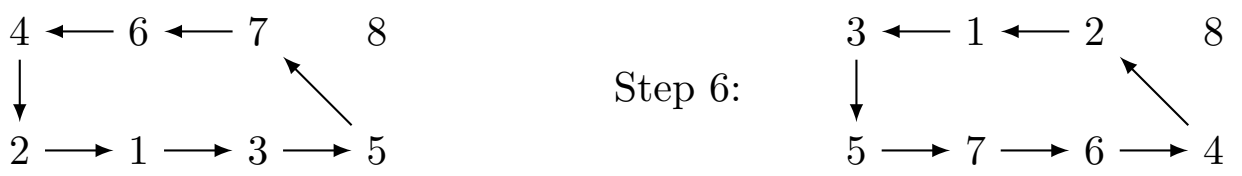

Step 3:

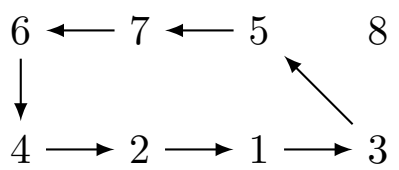

Step 7:

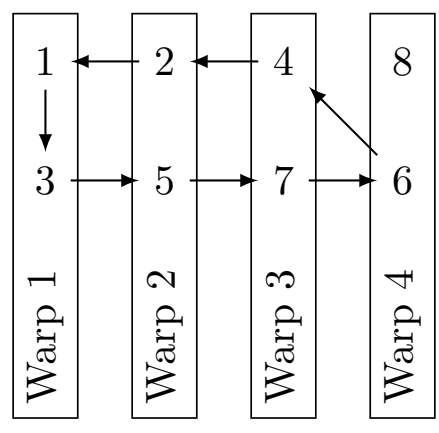

Figure 3.5: Distribution of column pairs to warps at each step of a sweep.

as I increase the number of threads assigned to the operation until matrix sizes of $64 \times 64$ reach the block limit of 1024 threads. To handle larger sizes, I must use a blocked version of the algorithm or the randomized SVD as shown in Sections 3.5 and 3.6, respectively.

\subsection{Global Memory One-Sided Block Jacobi}

When I can no longer store the entire matrix in shared memory, I have to operate on the matrix in the slower global memory. Instead of repeatedly reading and updating the columns one at a time, block algorithms that facilitate cache reuse have been developed [53, 54, 55]. The main benefit of the block Jacobi algorithm is its high degree of parallelism; however, since I implement a batched routine for independent operations, I will use the serial block Jacobi algorithm for individual matrices and rely on the parallelism of the batch processing. The parallel version, where multiple blocks are processed simultaneously, can still be used when the batch size is very small, but I 


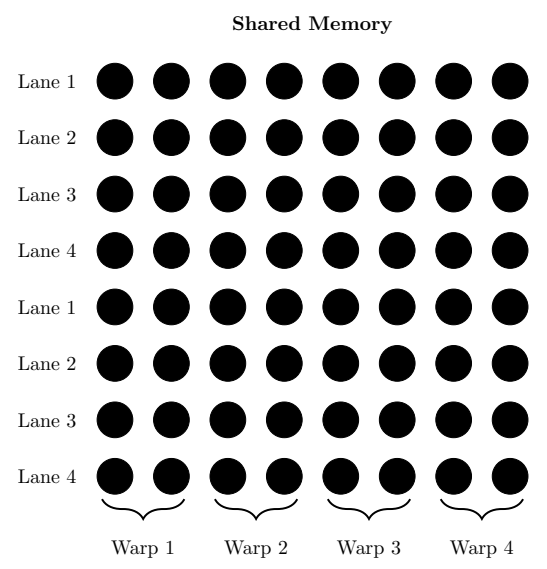

(a) Matrix columns assigned in pairs to multiple warps and stored in shared memory.

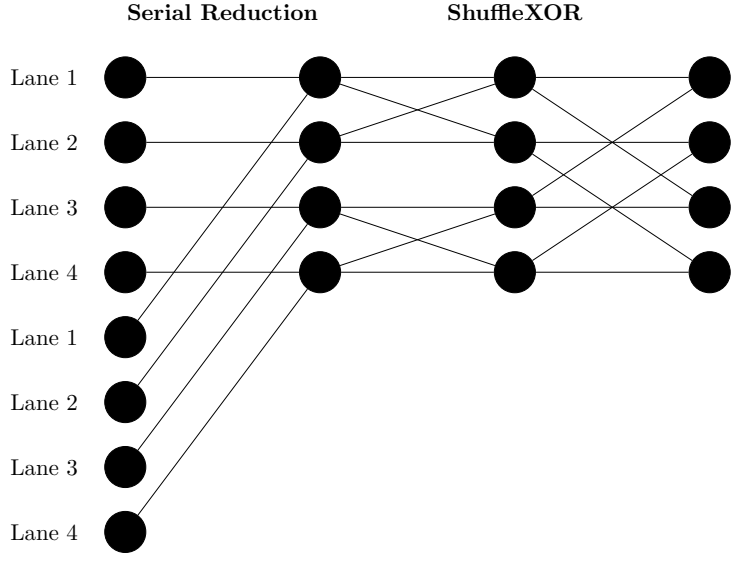

(b) Parallel reduction of a column of data in shared memory using register shuffles after an initial serial reduction step.

Figure 3.6: Shared memory kernel implementation details.

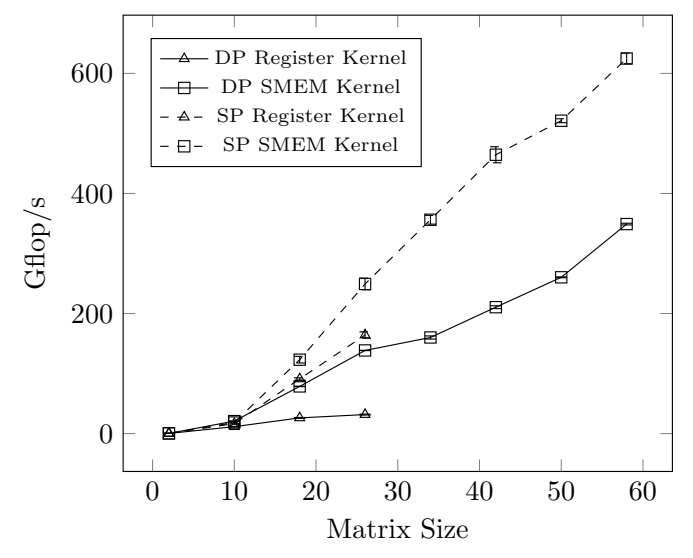

(a) Shared memory kernel performance in GFLOPs/s compared to the register kernel.

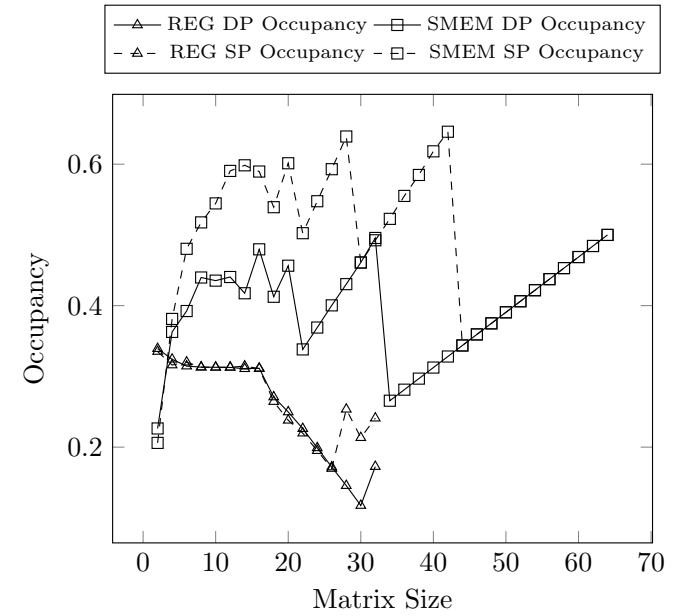

(b) Comparison of the occupancy achieved by the register and shared memory kernels.

Figure 3.7: Performance of the batched shared memory SVD on a P100 GPU for 1000 matrices of varying size in single and double precision arithmetics. 
will focus on the serial version. This section discusses the implementation details for two global memory block Jacobi algorithms that differ only in the way block columns are orthogonalized and compare their performance with parallel streamed calls to the cuSOLVER 8 [56] library routines.

\subsubsection{Gram Matrix Block Jacobi SVD}

The block Jacobi algorithm is very similar to the vector-oriented Algorithm2, orthogonalizing pairs of blocks columns instead of vectors. The first method of orthogonalizing pairs of block columns is based on the SVD of their Gram matrix. During the $p$-th sweep, each pair of $m \times k$ block columns $A_{i}^{(p)}$ and $A_{j}^{(p)}$ is orthogonalized by forming a $2 k \times 2 k$ Gram matrix $G_{i j}^{(p)}=\left[A_{i}^{(p)} A_{j}^{(p)}\right]^{T}\left[A_{i}^{(p)} A_{j}^{(p)}\right]=A_{i j}^{(p)^{T}} A_{i j}^{(p)}$ and generating a block rotation matrix $U_{i j}^{(p)}$, computed as the left singular vectors of $G_{i j}^{(p)}$ (or equivalently its eigenvectors, since it is symmetric positive definite). Updating $A_{i j}^{p+1}=A_{i j}^{p} U_{i j}^{(p)}$ orthogonalizes the block columns, since

$$
A_{i j}^{p+1^{T}} A_{i j}^{p+1}=U_{i j}^{(p)^{T}} A_{i j}^{p T} A_{i j}^{p} U_{i j}^{(p)}=U_{i j}^{(p)^{T}} G_{i j}^{(p)} U_{i j}^{(p)}=\Lambda_{i j}^{p},
$$

where $\Lambda_{i j}^{p}$ is a diagonal matrix of the singular values of $G_{i j}^{(p)}$. Orthogonalizing all pairs of block columns until the entire matrix is orthogonal will give the left singular vectors as the normalized columns and the singular values as the corresponding column norms. If the right singular vectors are needed, one can accumulate the action of the block rotation matrices on the identity matrix. For my batched implementation, I use highly optimized batched syrk and gemm routines from MAGMA to compute $G$ and to apply the block rotations, while the SVD is computed by the shared memory batched kernel. Since different matrices will converge in different numbers of sweeps, I keep track of the convergence of each operation $l$ by computing the norm $e_{l}$ of the off-diagonal entries of $G$ scaled by its diagonal entries. While this term is an inexact 
approximation of the off-diagonal terms of the full matrix in each sweep, it is still a good indication of convergence and will cost at most an extra cheap sweep, since the final sweep will not actually perform any rotations within the SVD of $G$. The entire batched operation will then converge when $e=\max e_{l}<\epsilon$, where $\epsilon$ is the convergence tolerance. This gives the Gram matrix path of the batched block Jacobi Algorithm 5 to compute the SVD of a batch of matrices in global memory. It is worth noting that the computation of the Gram matrix can be optimized by taking advantage of the special structure of $G$, but since the bulk of the computation is in the SVD of $G$, it will not result in any significant performance gains.

\subsubsection{Direct Block Jacobi SVD}

The Gram matrix method is an indirect way of orthogonalizing block columns and may fail to converge if the matrix is very ill-conditioned. Ill-conditioned matrices can be handled by directly orthogonalizing the columns using their SVD. Since the block columns are rectangular, I first compute their QR decomposition followed by the SVD

of the triangular factor $R$. Overwriting the block column $A_{i j}^{p}$ by the orthogonal factor $Q$ and multiplying it by the left singular vectors of $R$ scaled by the singular values will give the new block column $A_{i j}^{p+1}$ :

$$
A_{i j}^{p}=Q_{i j}^{p} R_{i j}^{p}=\left(Q_{i j}^{p} U_{i j}^{p} \Sigma_{i j}^{p}\right) V_{i j}^{p T}=A_{i j}^{p+1} V_{i j}^{p T}
$$

If the right singular vectors are needed, one can accumulate the action of $V_{i j}^{p}$ on the identity matrix. For this batched implementation, I use the batch QR routine developed in Section 3.2 and gemm routines from MAGMA to multiply the orthogonal factor by the left singular vectors, while the SVD is computed by the shared memory batched kernel. The same convergence test used in the Gram matrix method can be used on the triangular factor, since the triangular factor should be close to a diagonal 


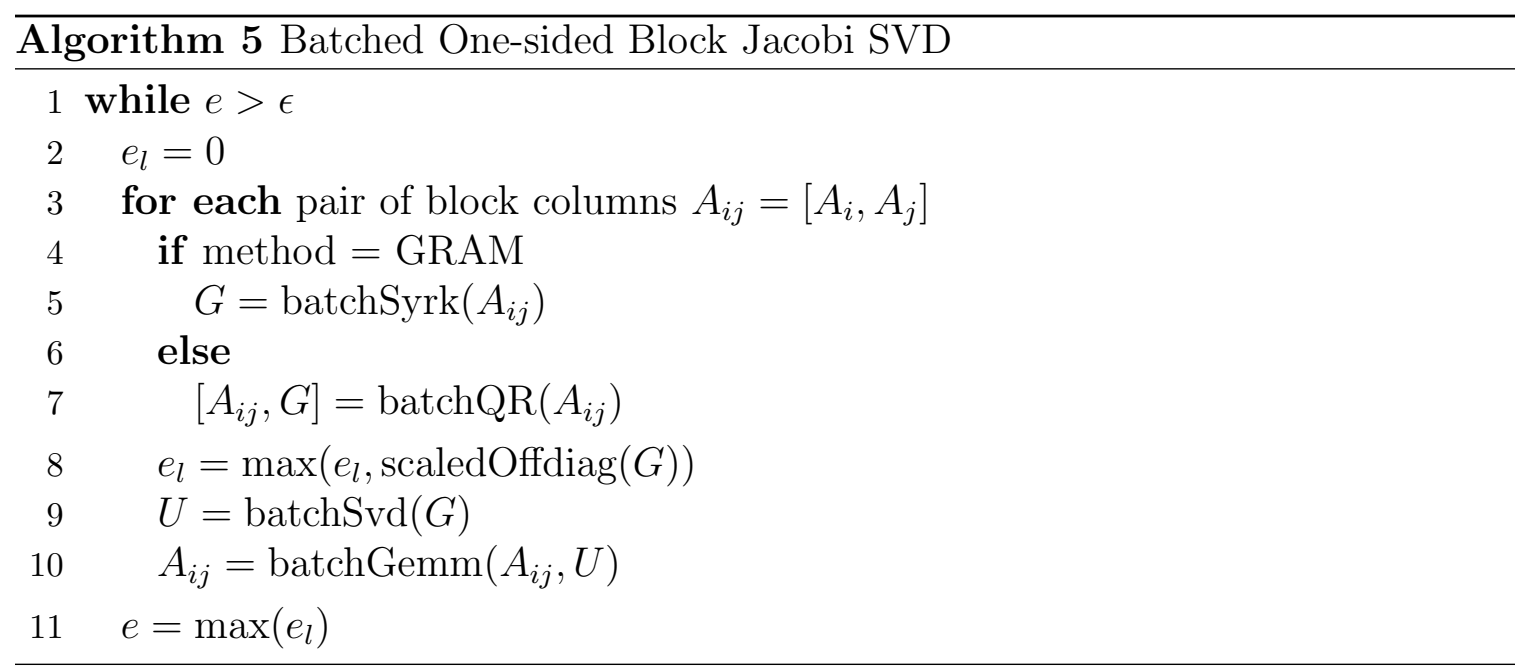

matrix if a pair of block columns are orthogonal. This yields the direct path of the batched block Jacobi Algorithm 5 to compute the SVD of a batch of matrices in global memory.

\subsubsection{Performance}

Figures $3.8 \mathrm{a}$ and $3.8 \mathrm{a}$ show the profiling of the different computational kernels involved in the batched block algorithms with a block width of 32, specifically percentages of total execution time for determining convergence and memory operations, matrix multiplications, QR decompositions and the SVD of the Gram matrix. For the Gram matrix approach, the SVD is the most costly phase, even for the larger operations, while the QR and SVD decompositions take almost the same time for the larger matrices in the direct approach. Figure 3.9a shows the performance of the batched block Jacobi SVD of 200 matrices using both methods and Figure $3.9 \mathrm{~b}$ compares the performance of my batched SVD routine with a batched routine that uses the cuSOLVER SVD routine using 20 concurrent streams on a P100 GPU. Increasing the number of streams for cuSOLVER showed little to no performance benefits, highlighting the performance limitations of routines that are bound by kernel launch overhead. The matrices are generated randomly using the latms LAPACK routine with a condition 
number of $10^{7}$. The Gram matrix approach fails to converge in single precision for these types of matrices, whereas the direct approach always converges; however the Gram matrix approach performs better when it is applicable for the larger matrices due to the strong performance of the matrix-matrix multiplications. The performance of the block algorithm can be improved by preprocessing the matrix using $\mathrm{QR}$ and LQ decompositions to decrease the number of sweeps required for convergence [57] as well as by adaptively selecting pairs of block columns based on the computed offdiagonal norms of their Gram matrices. These changes are beyond the scope of this dissertation and will be the focus of future work.

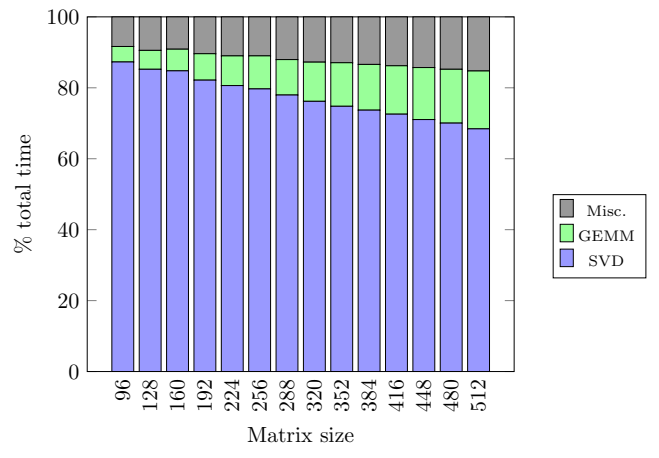

(a) Gram Matrix batched block Jacobi SVD profile.

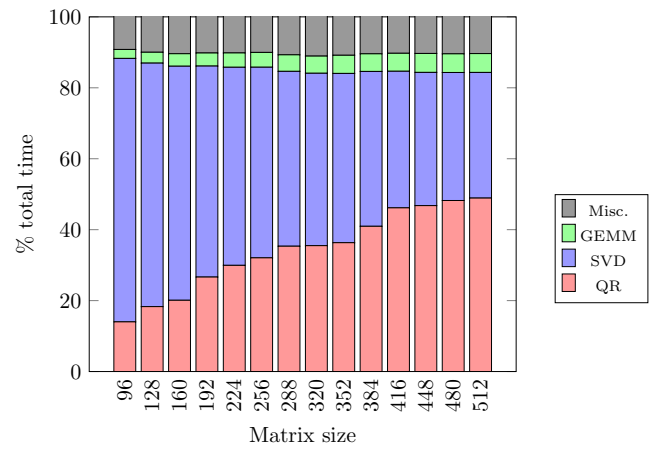

(b) Direct batched block Jacobi SVD profile.

Figure 3.8: Profile of the different phases of the block Jacobi SVD for 200 matrices of varying size on a P100 GPU in double precision. Single precision exhibits similar behavior.

\subsection{Fixed Rank Randomized SVD}

As mentioned at the beginning of this chapter, one is often interested in a rank- $k$ approximation of a matrix $A \approx \tilde{U} \tilde{S} \tilde{V}$. This approximation can be computed by first determining the singular value decomposition of the full $m \times n$ matrix $A$ and then truncating the $n-k$ smallest singular values with their corresponding singular vectors. However, when the matrix has low numerical rank $k$, one can obtain the approximation using fast randomization methods [42]. This section will discuss some 


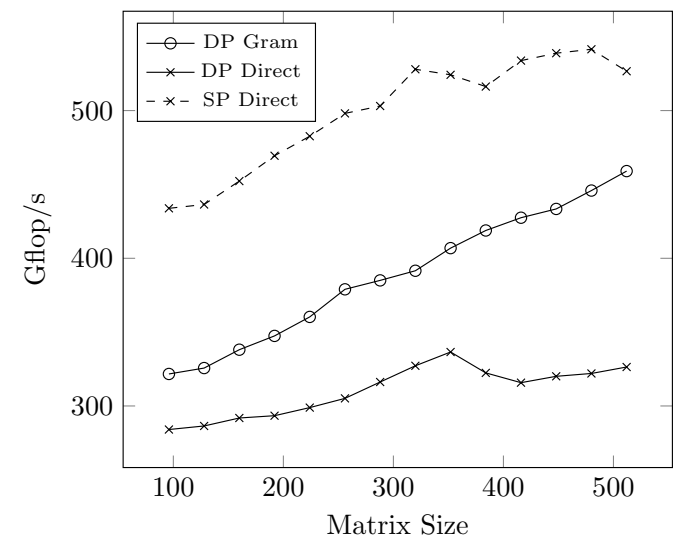

(a) Batched block Jacobi SVD performance.

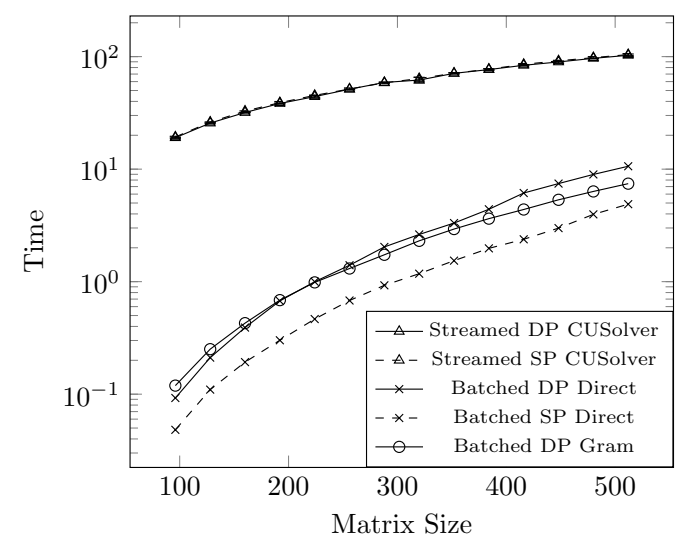

(b) Comparison between streamed cuSOLVER and the batched block Jacobi.

Figure 3.9: Batched block Jacobi performance for 200 matrices of varying size on a P100 GPU in single and double precision arithmetics.

details of the algorithm and compare its performance with the full SVD using the one-sided block Jacobi kernel.

\subsubsection{Implementation}

When the singular values of a matrix decay rapidly, an approximate SVD can be computed using a simple two-phase randomization method:

1. The first phase determines an approximate orthogonal basis $Q$ for the columns of $A$, ensuring that $A \approx Q Q^{T} A$. When the numerical rank $k$ of $A$ is low, one can be sure that $Q$ has a small number of columns as well. By drawing $k+p$ sample vectors $y=A w$ from random input vectors $w$, one can obtain a reliable approximate basis for $A$ [42] which can then be orthogonalized. This boils down to computing a matrix $Y=A \Omega$, where $\Omega$ is a $n \times(k+p)$ random Gaussian sampling matrix, and then computing the $\mathrm{QR}$ decomposition of $Y=Q R_{y}$, where $Q$ is the desired approximate orthogonal basis.

2. The second phase uses the fact that $A \approx Q Q^{T} A$ to compute a matrix $B=$ $Q^{T} A$ so that now $A \approx Q B$. Forming the SVD of $B=U_{B} S V^{T}$ finalizes the 


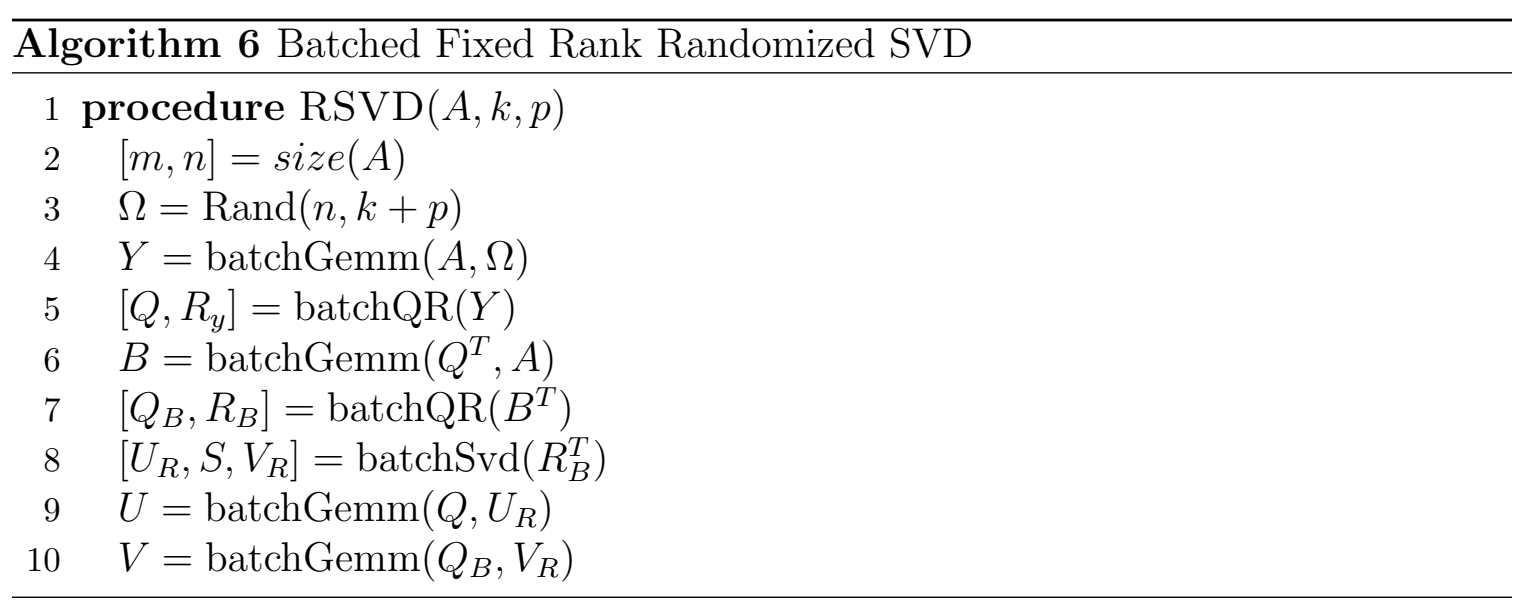

approximation $A \approx Q U_{B} S V^{T}=U S V^{T}$. For the wide $(k+p) \times n$ matrix $B$, one can first compute a QR decomposition of its transpose, followed by the SVD of the upper triangular factor.

Algorithm 6 shows that the core computations for the randomized method are matrixmatrix multiplications, QR decompositions, and the singular value decompositions of small matrices. Using the batched routines from the previous sections, it is straightforward to form the required randomized batched SVD. More robust randomized SVD algorithms would employ randomized subspace iteration methods to obtain a better basis $Q$ for the columns of $A$ and rely on these same core kernels, but will not be further discussed here.

\subsubsection{Performance}

Figure 3.10 shows the profiling of the different kernels used in the randomized batched routine for determining the top 64 singular values and vectors of randomly generated low rank matrices using the latms LAPACK routine. The miscellaneous portion includes random number generation using the CURAND library's default random number generator and a Gaussian distribution, batched transpose operations and memory operations. One can see that the various kernels play almost equally important roles in the performance of the randomized routine as the matrix size grows while keeping 
the computed rank the same. Figure 3.11a shows the performance of the batched randomized SVD of 200 operations and Figure 3.11b compares the runtimes of the direct block one-sided Jacobi routine with the randomized SVD on a P100 GPU for the same set of matrices, showing that significant time savings can be achieved even for relatively small blocks.

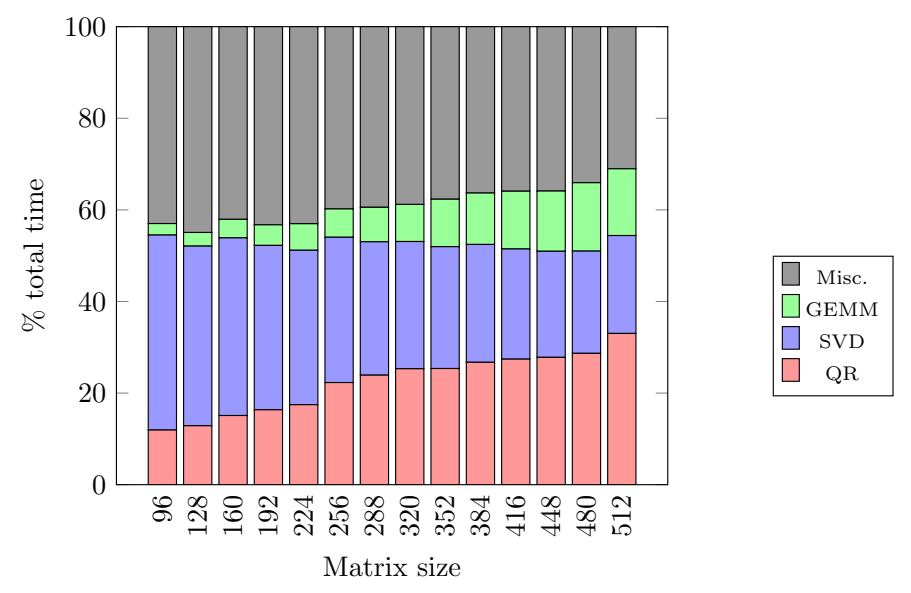

Figure 3.10: Profile of the different phases of the batched randomized SVD for 200 matrices of varying size on a P100 GPU in double precision. Single precision exhibits similar behavior.

\subsection{Adaptive Randomized Approximation}

While the adaptive randomized approximation (ARA) can determine the appropriate rank for a specified error tolerance, most of the computations in Algorithm 4 , such as the sampling at line 17, the projections at line 20 and the norm computations at line 21, are memory bound operations, while the computations in the fixed rank Algorithm 3 are mostly compute bound. When implemented on the GPU, a platform that greatly favors high arithmetic intensity, the fixed rank algorithm has a clear advantage in performance and blocked versions of randomized algorithms have been shown to have superior runtime performance and better accuracy [58]. In this section, I will discuss the details of a blocked version of the ARA and its batched GPU implementation and 


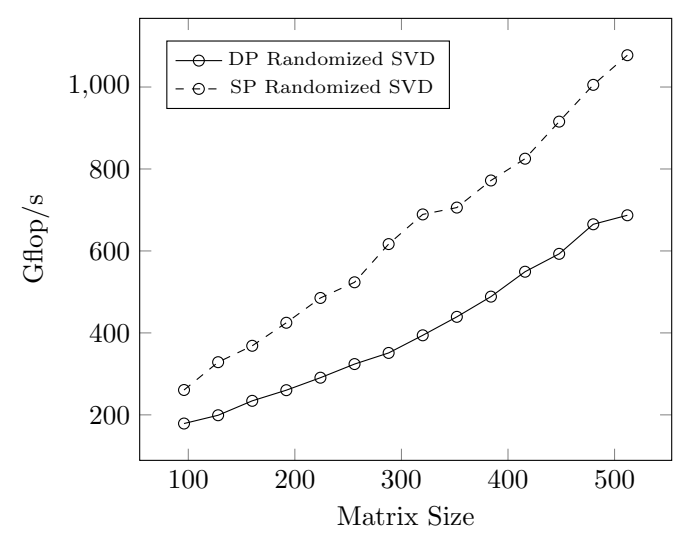

(a) Batched randomized SVD performance.

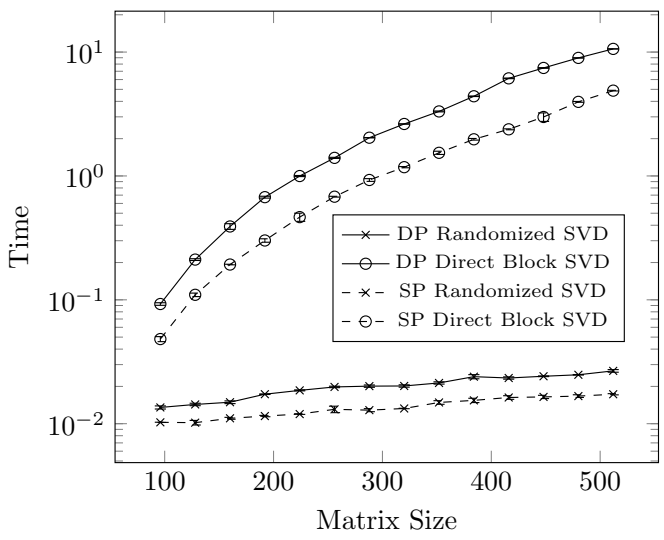

(b) Comparison between the batched block Jacobi and the batched randomized SVD.

Figure 3.11: Batched randomized SVD performance for 200 matrices of varying size on a P100 GPU in single and double precision for the first 64 singular values and vectors.

compare its performance with the batched SVD routines developed in previous work [43].

\subsubsection{Blocked version}

Processing blocks of samples allows the use of BLAS Level 3 routines instead of the memory bound BLAS Levels 2 and 1 operations, greatly increasing the arithmetic intensity of the adaptive algorithm. The blocked algorithm is very similar to Algorithm 4. however, instead of sampling one vector at a time, the blocked algorithm produces multiple samples simultaneously and orthogonalizes using a blocked version of Gram Schmidt (BGS) [45]. While this comes with the cost of potentially performing more work by oversampling, the performance gains more than compensate.

The original BGS algorithm still requires that blocks of columns $Y$ be orthogonalized. This can be achieved either using the unblocked GS algorithm where high performance can be difficult to achieve due to the repeated norm computations or Householder QR factorization [43]. In the absence of efficient batched QR routines for the tall matrices produced by large problems, I turn to other methods that would 
allow the use of other high performance routines. Taking advantage of the fact that I already perform one reorthogonalization step as well as the fact that the algorithm should stop sampling when the norms of the vectors reach the error threshold, I use Cholesky QR [59] to perform the orthogonalization instead, allowing the use of much higher performance kernels. This involves computing a Gram matrix $G=Y^{T} Y$, computing the Cholesky decomposition of $G=R^{T} R$, and finally performing a triangular solve on the input columns $Y=Y R^{-1}$ to produce the orthogonal columns. Cholesky QR is unfortunately numerically unstable due to the squaring of the condition number when forming the Gram matrix, causing the method to fail in single precision when a low relative error is needed. To overcome this, I compute the Gram matrix and the Cholesky factorization in mixed precision, reading in the data in single precision and computing in double precision. This gives the benefits of the additional accuracy of double precision while keeping the original data in single precision and has been shown to greatly improve the numerical stability of the method [60]. When computing in double precision, I assume that the requested error threshold will not be extremely small, so I do not emulate quad precision [61, 62] and instead compute in the same precision as the input. In practice this has allowed approximations with relative thresholds as low as $10^{-10}$, which is significantly lower than the needs of the motivating applications. If greater accuracy is required, quad precision may be needed to stabilize the double precision Cholesky QR.

\subsubsection{Implementation}

The non-uniform batched ARA GPU implementation of Algorithm 7 builds on several high performance non-uniform batched kernels: specifically non-uniform matrixmatrix multiplication from the MAGMA library [63], random number generation from the CURAND library [64], as well as many kernels that I develop for this dissertation which I discuss briefly here. To form the Gram matrices, I implement a 
batched mixed precision syrk GPU kernel that reads in the matrix data in the original precision and performs the computations in double precision. This kernel loads the matrix from global memory one block at a time into shared memory and stores and computes the output matrix in registers. It is specifically optimized for the case of transpose syrk of a tall and skinny matrix. I also implement a batched mixed precision potrf GPU kernel to compute the Cholesky factorization of the Gram matrices, outputting the triangular factor in the original precision while computing in double precision. Assigning multiple warps, where a warp is a group of 32 threads that execute instructions in parallel, to process multiple columns, the input matrix is loaded from global memory and factorized entirely in shared memory. This routine also stores and updates the diagonal of the triangular factor as it goes through the reorthogonalization process since those values are used to determine the convergence of the operation (the equivalent of line 21 in Algorithm 4). These kernels can easily be extended to emulate quad precision input/output if additional accuracy is required. The kernel batchSetSvec determines whether an operation has converged by keeping track of the number of vectors with sufficiently small norms, while the kernel batchSetSamples determines how many samples to take for each operation in the batch as well as whether all operations have converged. Finally, sampling is done using a black box routine that is provided by the user and could be anything from a simple matrix-matrix multiplication to a specialized routine that only produces the output of multiple matrix-vector products.

\subsubsection{Performance}

I show the effectiveness and performance of ARA when compared with the full SVD for generating an approximation of a batch of 1000 randomly generated numerically low rank matrices. The matrices are generated using the lapack routine latms and the singular values are generated using the expression $s_{i}=t e^{-\alpha i}$ to simulate the 


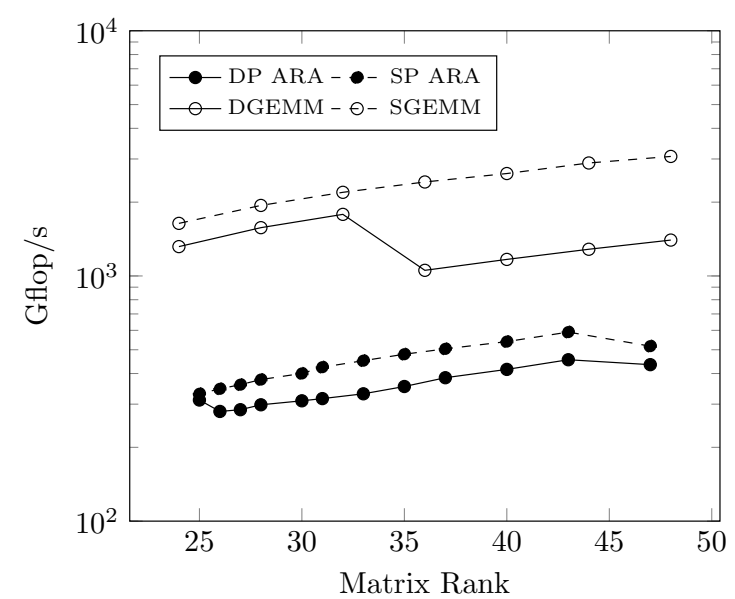

(a) Batched ARA performance in single and double precision for a batch of 1000 matrices of varying rank and fixed size $(128 \times 128)$. Performance of batched gemm included for reference.

Figure 3.12: Batched ARA performance for a precision of $10^{-6}$.

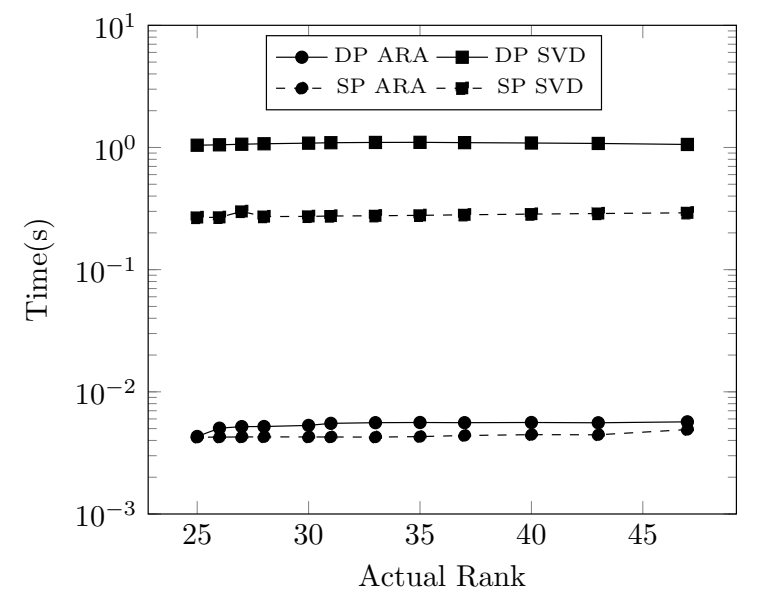

(a) Time for the SVD and ARA of a batch of 1000 matrices in single and double precision with varying rank and fixed size $(128 \times 128)$. with varying size and fixed rank (47).

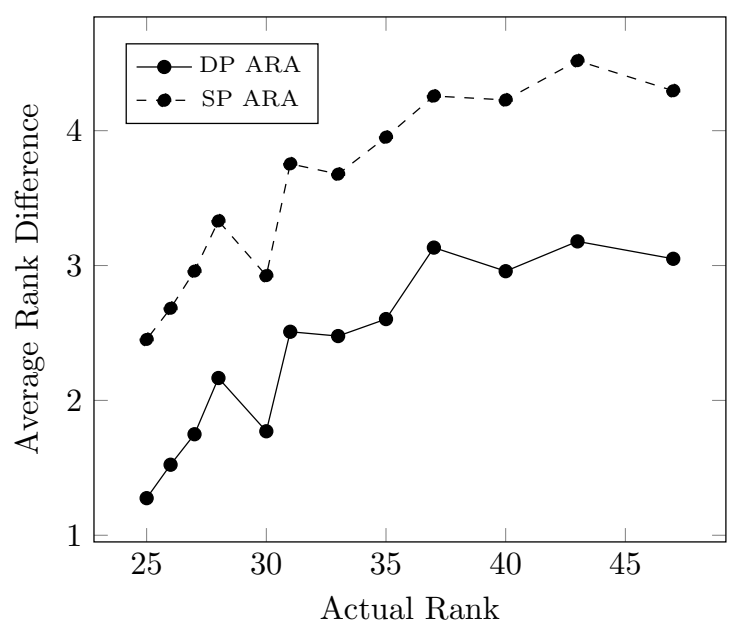

(b) Average difference in numerical rank and detected rank in single and double precision for a batch of 1000 matrices.

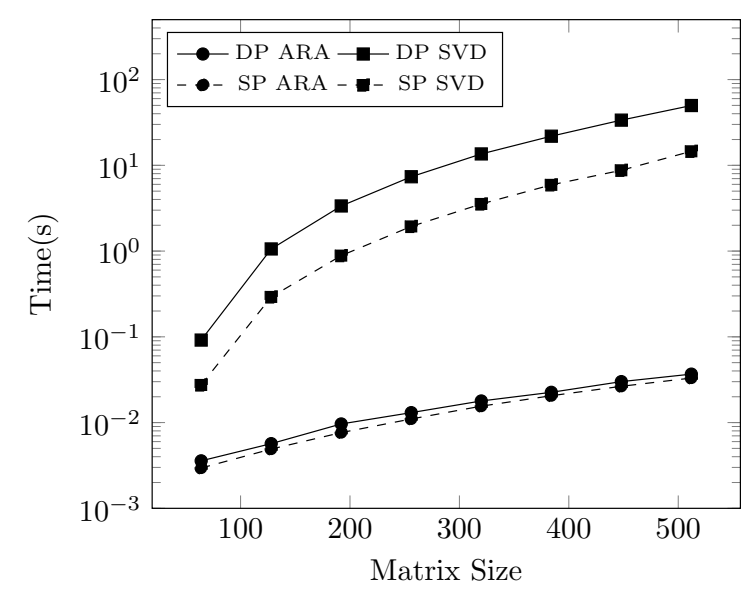

(b) Time for the SVD and ARA of a batch of 1000 matrices in single and double precision

Figure 3.13: Comparison of batched ARA and SVD kernels 
expected rapid decay, where $t$ is a random scaling factor and $\alpha$ controls the speed of the decay. Increasing $\alpha$ leads to faster singular value decay, producing different approximation ranks $k$ for the chosen error threshold $\epsilon=10^{-6}$. For the following results, I use a block size $b s$ of 32 which was empirically determined to produce the best performance (not surprising since the warp size is 32) and $r$, the number of required consecutive small vectors for convergence, was set to 10. Figure 3.12a shows the performance of the batched ARA routine in GFLOP/s for a batch of 1000 dense $128 \times 128$ matrices for different values of $\alpha$. ARA achieves a peak performance of $450 \mathrm{GFLOP} / \mathrm{s}$ in double and $600 \mathrm{GFLOP} / \mathrm{s}$ in single precision as opposed to the theoretical peak performance of $4.7 \mathrm{TFLOP} / \mathrm{s}$ in double and $9.3 \mathrm{TFLOP} / \mathrm{s}$ in single precision of the P100 GPU. In comparison, the batched-GEMM peformance on the $\mathrm{P} 100$ is $1.5 \mathrm{TFLOP} / \mathrm{s}$ in double and $3 \mathrm{TFLOP} / \mathrm{s}$ in single precision for a batch of 1000 matrix-matrix products of $128 \times 128$ matrices with $128 \times k$ matrices, which represent the practical peaks for the performance of the batched operations presented here. The flops for the batch are computed as the sum of operations performed for each matrix as determined by the unblocked Algorithm 4. The dip in performance from rank 43 to rank 47 is due to the need for an additional block of samples to satisfy the convergence criteria, producing a relatively large amount of unneeded samples. Figure $3.12 \mathrm{~b}$ shows the average difference in numerical rank as determined by the truncation threshold $\epsilon$ and the decay of the singular values and the rank $k$ detected by ARA for the aforementioned batch of matrices. The difference increases as the actual truncation rank increases due to slower singular value decay and can be reduced either by subspace iteration or by performing the SVD of the very small $k \times k$ matrices, as discussed in Section 3.1.3. It is worth noting that while the average difference in the rank for each batch may be relatively small, the batch may contain some matrices with a relatively large rank difference which may limit the method's usefulness in some situations. Figure $3.13 \mathrm{a}$ compares the time taken to compute the full SVD with the 
time for the ARA of the aforementioned batch of matrices. Since I compute the full SVD and then truncate, the time for each batch is almost the same and is up to $240 \mathrm{x}$ slower than the ARA. This is an expected result, since the blocked Jacobi SVD used here [43] is an $O\left(n^{3}\right)$ algorithm focusing on kernels that are not particularly efficient, while the ARA is $O\left(k n^{2}\right)$ for dense matrices of rank $k$ and size $n \times n$ focusing on highly efficient matrix-matrix kernels. Figure 3.13b compares the times for a batch of 1000 matrices of various sizes and fixed rank of 47, highlighting the difference in complexity of the two algorithms.

\subsection{Miscellaneous Batched Operations}

While I also developed other batched operations, such as matrix vector products, matrix transpose, copy of matrix blocks, full pivoted Cholesky and more, their implementations are relatively straightforward and as such do not merit much elaboration. They mostly follow a simple thread and block allocation, where a thread is assigned per row of the input or to each entry in the matrix. I briefly mention some points of interest for some of the implementations:

- Batched transpose matrix vector product: a single warp is assigned to a column regardless of the number of rows in the matrix in an effort to increase the efficiency of the reductions. Matrix entries are loaded by column, 32 (corresponding to the warp size) entries at a time to make sure loads are coalesced. Parallelism is extracted by assigning multiple warps to an operation, with each warp assigned to multiple columns. The number of warps assigned is chosen dynamically based on the number of operations in the batch to increase performance when the batch size is small.

- Batched matrix transpose: matrix blocks are loaded by coalesced reads into shared memory that is padded to avoid memory bank conflicts and then stored 
into global memory in coalesced writes.

- Full pivoted Cholesky factorization: I load the entire block into shared memory and factorize up to the shared memory limit of $64 \times 64$ matrix in double precision. A thread group is equal to the number of rows of the matrix, and multiple groups are assigned to the update of the trailing matrix, with a single group responsible for computing the updates. 


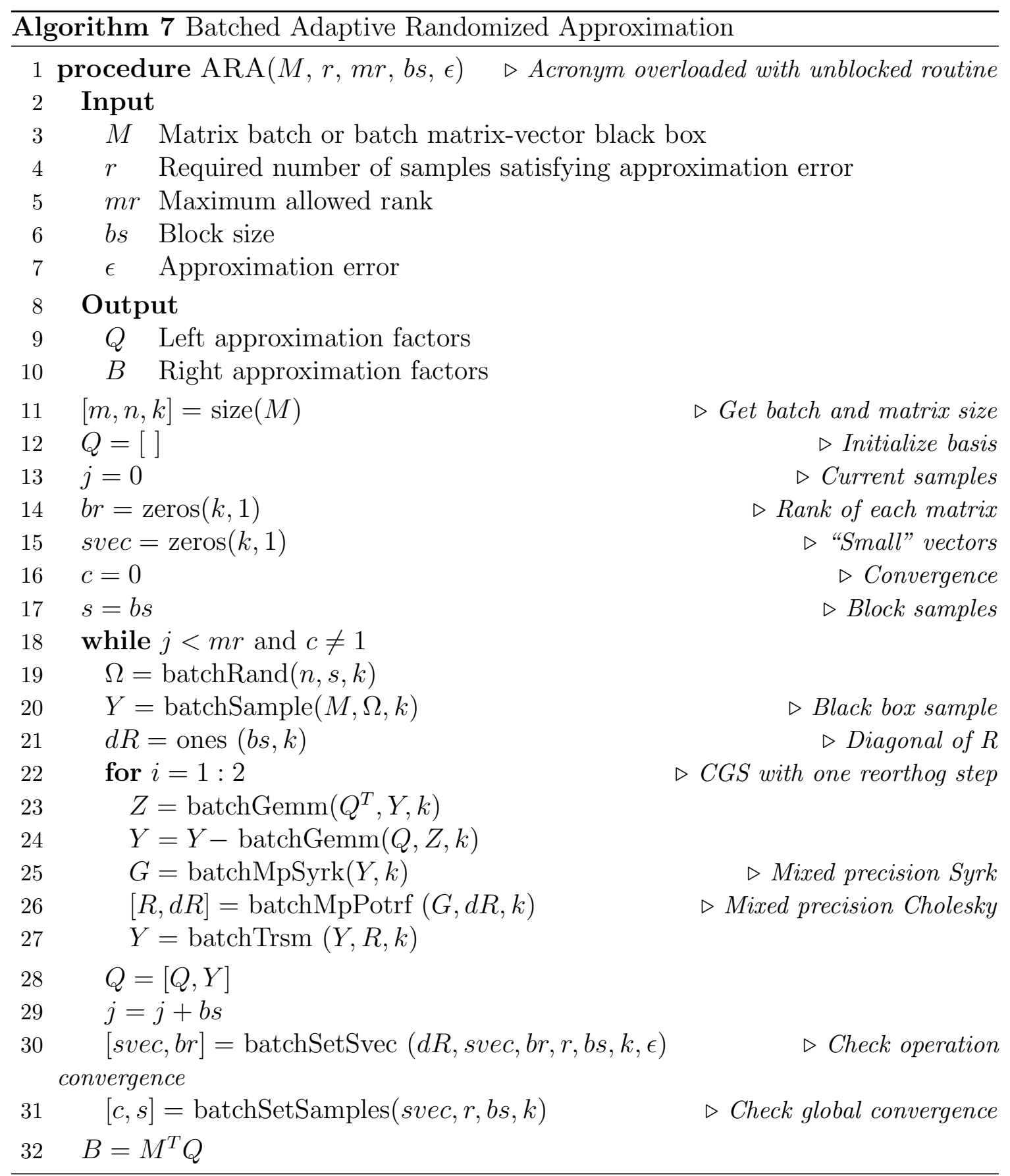




\section{Chapter 4}

\section{Hierarchical Matrix Vector Multiplication}

The product of a matrix with a vector is a key component in many important iterative algorithms, such as Krylov subspace methods and, as shown in Chapter 3 , the adaptive randomized approximation of low rank matrices. I generalize the ARA algorithm to the construction of a hierarchical matrix in Chapter 6, where repeated matrix vector products are required. In this chapter, I describe the different phases of an hgemv algorithm as a generalization of the standard BLAS gemv routine, where an input vector $x$ is multiplied by a hierachical matrix to produce an output vector $y$, and their efficient GPU implementations.

Given that a hierachical matrix can be expressed as the sum of its dense blocks with its low rank blocks we have $y=A x=A_{D} x+A_{L R} x$, where $A_{D}$ is a matrix consisting only of the dense blocks and $A_{R}$ contains all the low rank blocks of $A$. Figure 4.1 shows an overview of the different phases of the algorithm. First, I compute the product of the dense blocks with the input vector using a block sparse matrix vector multiplication routine. The product of the dense blocks with the input vector is computed via a block sparse matrix vector multiplication routine. The product of the low rank blocks with the input is then computed in three additional phases: an upsweep phase, a multiplication phase, and a downsweep phase as illustrated in Figure 4.1. For intuition, we may think of this algorithm as the hierarchical generalization of the way we multiply a regular dense low rank matrix, $U S V^{T}$, by a vector $x$. In this case we would do it in three phases: first apply the transpose of $V$ to $x$, then multiply the small resulting product by $S$, and then apply $U$ to obtain 


\begin{tabular}{ll}
\hline Symbol & Description \\
\cline { 1 - 2 }$n$ & matrix size \\
$k^{l}$ & size of dense blocks \\
$\mathcal{U}, \mathcal{V}$ & maximum rank of blocks in matrix tree at level $l$ \\
$U, V$ & block row and column basis trees \\
$E, F$ & bases at the leaf level of $\mathcal{U}$ and $\mathcal{V}$ \\
$\mathcal{S}$ & transfer matrices for the $\mathcal{U}$ and $\mathcal{V}$ bases, respectively \\
$i, j\left(\right.$ or $i_{1}, i_{2}$, etc. $)$ & matrix quadtree of coupling matrix blocks \\
$i^{+}$ & indices of block rows and block columns respectively \\
$x(i)$ & parent of a node $i$ \\
$\hat{x}, \hat{y}$ & sub-vector of a vector $x$ corresponding to the index set $i$ \\
$U_{\|}, E_{\|}$, etc. & vectors defined at every level in the basis trees \\
\hline
\end{tabular}

Table 4.1: Notation used for the hierarchical matrix tree algorithms.

the final product. The hierarchical analogue first applies the transpose of the bases of all levels of $\mathcal{V}$ to $x$ by sweeping up the tree to compute the nodes of a vector tree $\widehat{x}$. The multiplication phase then computes a vector tree $\widehat{y}$ where each node in $\widehat{y}^{l}$ is the product of a block row of coupling matrices in $S^{l}$ with the corresponding nodes of $\widehat{x}^{l}$. The nodes of $\widehat{y}^{l}$ represent the level's partial contribution to the output vector expressed in the row basis $U^{l}$. Finally, a downsweep phase expands the nodes of $\widehat{y}$, multiplying them by the bases from the corresponding levels of $\mathcal{U}$ to produce the final output vector $y$. It is also worth noting that these phases of the hgemv computation are very closely related to the phases of the fast multipole method, of which $\mathcal{H}^{2}$-matrices can be regarded as an algebraic generalization [65].

Table 4.1 summarizes the symbols used in the description of the tree algorithms on hierarchical matrices.

\subsection{Upsweep Phase}

The upsweep computes a tree $\widehat{x}$ as the products of the transposed nodes of the column basis $V$ with the input vector $x$; i.e., $\widehat{x}_{j}^{l}=V_{j}^{l^{T}} x(j)$ for all nodes $j$ within a level $l$, with $l$ in $0 \cdots q$. This process is trivial for the leaves since they are stored explicitly; 


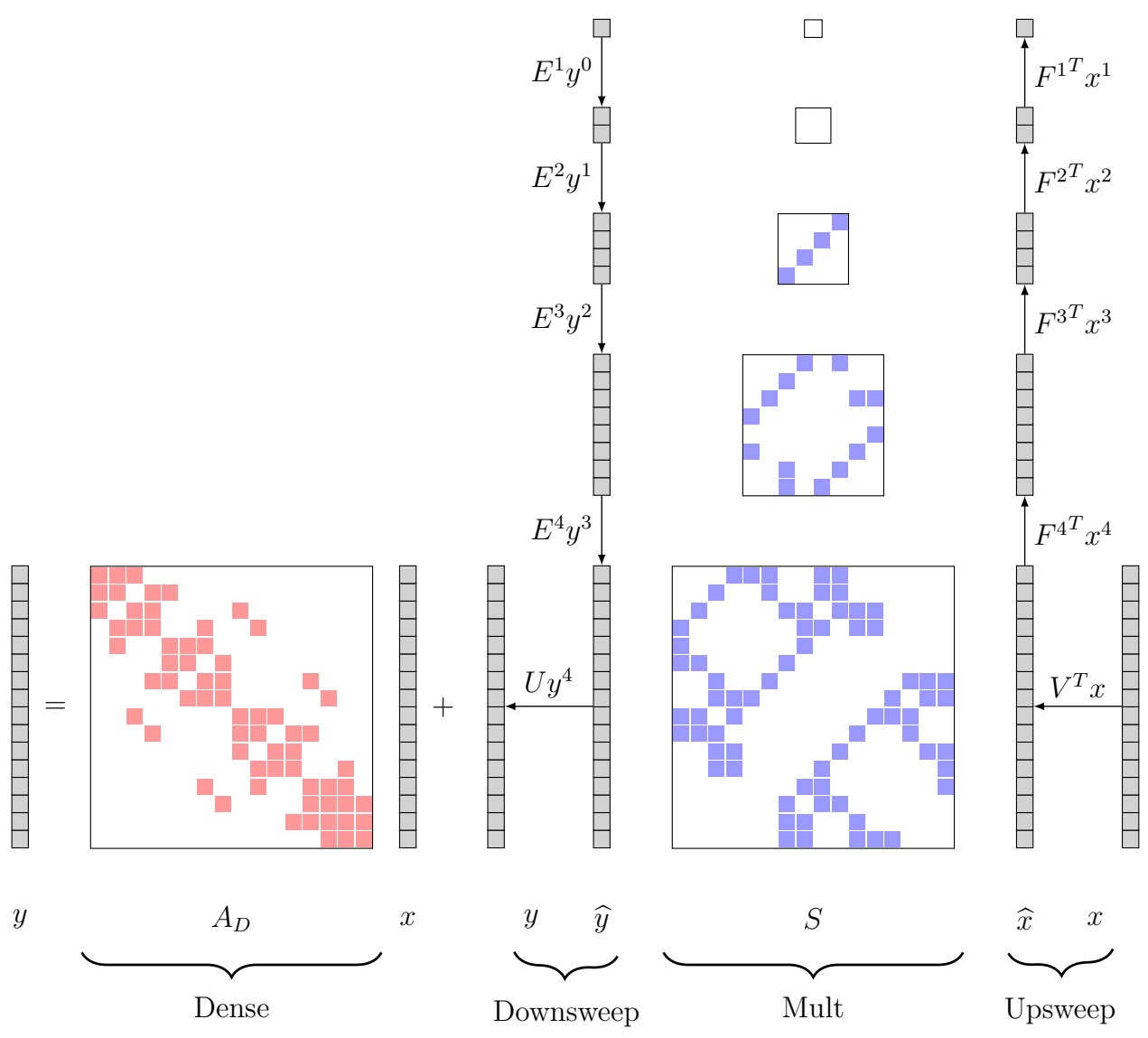

Figure 4.1: Overview of the hgemv. Computation with the low rank blocks is split into three phases: upsweep, multiplication, and downsweep. The upsweep computes a tree $\hat{x}$ from the input vector $x$ by first projecting it to the basis leaves and then sweeping up the tree using the transfer matrices. $\hat{x}$ is then fed into the block sparse multiplication phase to produce the $\hat{y}$ tree. Finally, the downsweep computes the output vector $y$ by first accumulating partial sums within each level of $\hat{y}$ using the transfer matrices. The leaf level of $\hat{y}$ then contains the full sums in terms of the basis leaves which are expanded to form the output vector $y$ and added to the results of the dense matrix-vector product to produce the final result.

however the inner nodes are expressed in terms of their children using the relationship defined in Eq. 1.1. For simplicity, let us consider a parent node $j^{+}$with two children $j_{1}$ and $j_{2}$. The node $\widehat{x}_{j^{+}}^{l-1}$ can be computed as:

$$
\widehat{x}_{j^{+}}^{l-1}=\left[\begin{array}{ll}
F_{j_{1}}^{l T} & F_{j_{2}}^{l T}
\end{array}\right]\left[\begin{array}{ll}
V_{j_{1}}^{l T} & \\
& V_{j_{2}}^{l} T
\end{array}\right]\left[\begin{array}{l}
x\left(j_{1}\right) \\
x\left(j_{2}\right)
\end{array}\right]=F_{j_{1}}^{l T} \widehat{x}_{j_{1}}^{l}+F_{j_{2}}^{l T} \widehat{x}_{j_{2}}^{l} .
$$




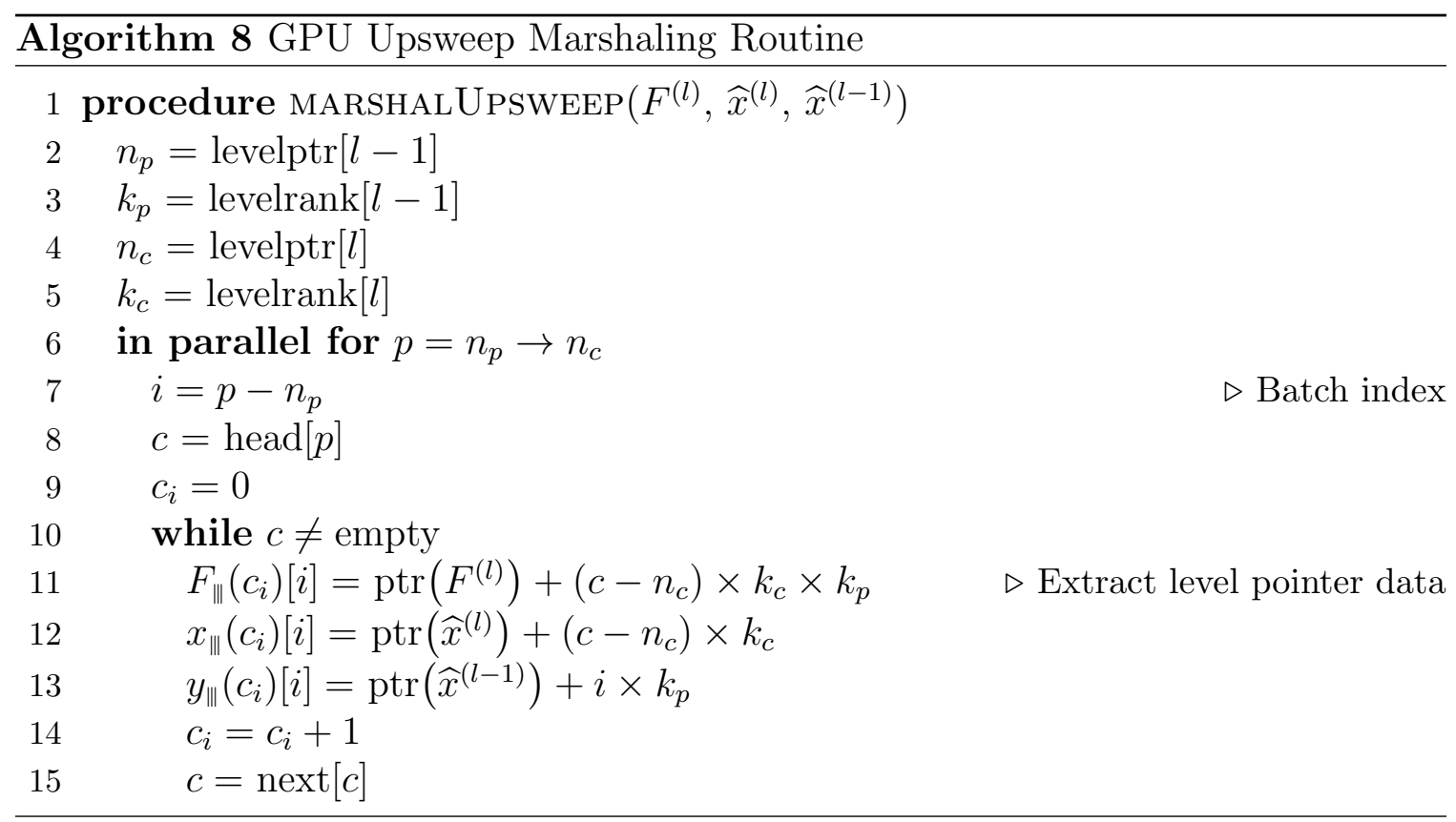

I can compute every node in $\widehat{x}$ by starting at the leaves and sweeping up the tree using the above equation. To avoid the prohibitive overhead of $\mathcal{O}(n)$ kernel launches required to execute this operation recursively on the GPU, I use the flattened tree structure described in Section 2.3 to compute $\widehat{x}$ level by level. The leaves are processed simply using a single batch matrix vector operation. Considering a binary tree for the basis trees, an upsweep kernel marshals the data for the operations in a level to generate two batches (one for each child), which are then executed by the batch matrix vector operation. Like all marshaling operations used in the rest of this thesis, this marshaling operation uses the flattened tree structure described in Section 2.3 to efficiently generate the necessary pointer data for the batched routines, the $F_{\|}$, $x_{\|}$and $y_{\|}$pointer arrays in this operation, using a single kernel call. This leads to Algorithm 8 for marshaling the upsweep operations and Algorithm 9 to compute $\widehat{x}$.

\subsection{Multiplication Phase}

The second phase of the computation builds a vector tree $\widehat{y}$, where each node $i$ in a level $l$ is the product of the block row $i$ of level $l$ of the coupling matrix tree with the 
corresponding nodes in $\widehat{x}$. This operation can be expressed as

$$
\widehat{y}_{i}^{l}=\sum_{j \in\left\{b_{i}\right\}} S_{i j}^{l} \widehat{x}_{j}^{l},
$$

where $b_{i}$ is the set of column indexes of the matrix blocks in the block row $i$. I could follow the same marshaling approach as the upsweep, but given the potential nonuniform distribution of blocks in different rows as well as the obvious similarity to a block sparse matrix vector multiplication, I opt to generate block sparse row (BSR) index data for the matrix tree. This data is efficiently generated once during the construction of the hierarchical matrix and stored per level of the matrix tree. Figure 4.2 shows an example of the block sparse row index data for a simple matrix. This leads to Algorithm 10 for the computation of $\widehat{y}$.

\subsection{Downsweep Phase}

After the multiplication phase, each level of the vector tree $\widehat{y}$ contains a partial contribution of the output vector $y$ expressed in terms of the nodes of the block row basis $U$ at that level. I can finalize the computation by expanding the nodes of $\widehat{y}^{l}$ at each level $l$ as:

$$
y(i)=y(i)+U_{i}^{l} \widehat{y}_{i}^{l}
$$

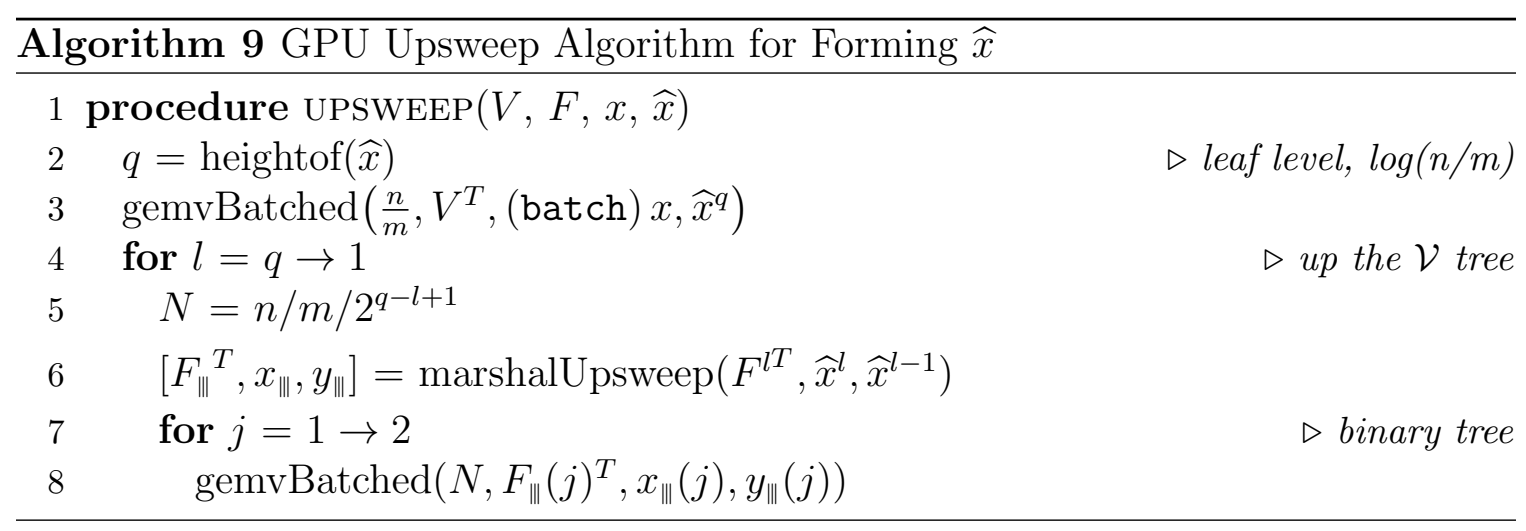




$$
A=\left[\begin{array}{lllllll}
a & c & & & e & g \\
b & d & & & f & h \\
& & i & k & & \\
& & j & l & & \\
& & & & m & o \\
& & & & n & p
\end{array}\right]
$$

\begin{tabular}{|c|c|c|c|c|c|c|c|c|c|c|c|c|c|c|c|c|}
\hline Values & $a$ & $b$ & $c$ & $d$ & $e$ & $f$ & $g$ & $h$ & $i$ & $j$ & $k$ & $l$ & $m$ & $n$ & $o$ & $p$ \\
\hline ColIdx & 1 & 3 & 2 & 3 & & & & & & & & & & & & \\
\hline RowPtr & 1 & 3 & 4 & 5 & & & & & & & & & & & & \\
\hline
\end{tabular}

Figure 4.2: The block sparse row storage for a $6 \times 6$ matrix with $2 \times 2$ blocks.

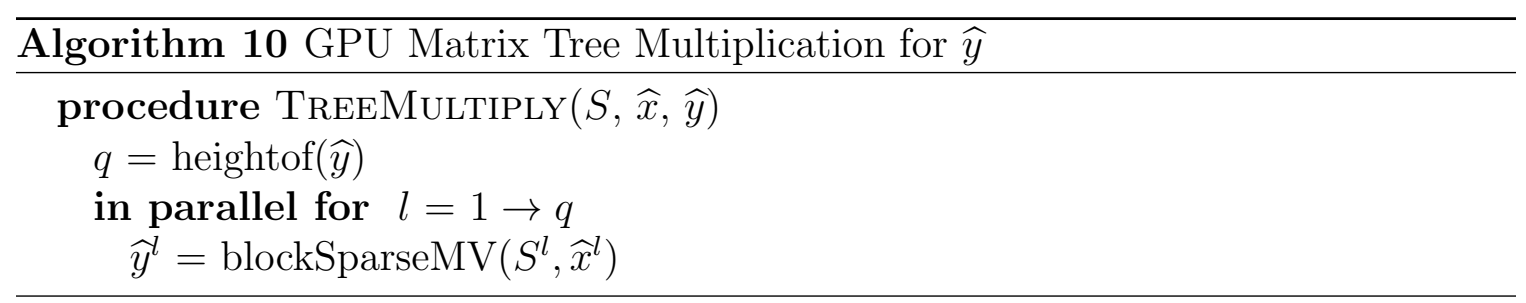

Since I lack an explicit representation of the inner nodes of the basis tree, I must use the nested basis property to express the partial sum of a level with its child level in terms of the basis nodes of the child level. Taking a parent node $i^{+}$at level $l-1$ and its two children $i_{1}$ and $i_{2}$ at level $l$, we have the partial sum:

$U_{i^{+}}^{l-1} \widehat{y}_{i^{+}}^{l-1}+\left[\begin{array}{c}U_{i_{1}}^{l} \widehat{y}_{i_{1}}^{l} \\ U_{i_{2}}^{l} \widehat{y}_{i_{2}}^{l}\end{array}\right]=\left[\begin{array}{cc}U_{i_{1}}^{l} & \\ & U_{i_{2}}^{l}\end{array}\right]\left[\begin{array}{c}E_{i_{1}}^{l} \widehat{y}_{i^{+}}^{l-1} \\ E_{i_{2}}^{l} \widehat{y}_{i^{+}}^{l-1}\end{array}\right]+\left[\begin{array}{l}U_{i_{1}}^{l} \widehat{y}_{i_{1}}^{l} \\ U_{i_{2}}^{l} \widehat{y}_{i_{2}}^{l}\end{array}\right]=\left[\begin{array}{cc}U_{i_{1}}^{l} & \\ & U_{i_{2}}^{l}\end{array}\right]\left[\begin{array}{l}E_{i_{1}}^{l} \hat{y}_{i^{+}}^{l-1}+\widehat{y}_{i_{1}}^{l} \\ E_{i_{2}}^{l} \hat{y}_{i^{+}}^{l-1}+\widehat{y}_{i_{2}}^{l}\end{array}\right]$.

Sweeping down $\widehat{y}$ and setting each node $\widehat{y}_{i}^{l}=\widehat{y}_{i}^{l}+E_{i}^{l} \widehat{y}_{i^{+}}^{l-1}$, the level $l$ at each step now also contains the partial sum of $y$ for all levels above $l$ expressed in the nodes of $U^{l}$. The leaf level will then contain the complete sum which is finally expanded into $y$. I follow the same approach as the upsweep, where each level is processed in parallel by first marshaling the operations and then executing using a batch matrix vector product. This leads to Algorithm 11 for computing $y$. The downsweep marshaling 
algorithm is structurally very similar to the upsweep marshaling routine described in Algorithm 8 and is omitted for brevity.

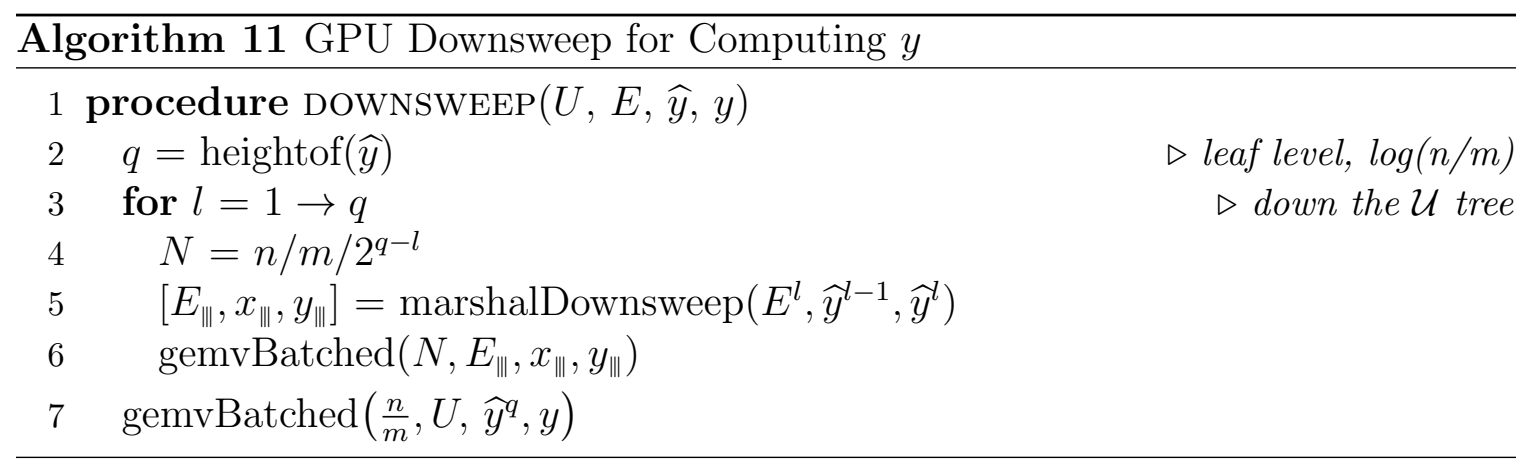

\subsection{Kernel Streaming}

The upper levels of the tree operations do not provide enough work to saturate the GPU and kernel overhead starts to impact performance for these levels. To overcome this, I can use streams to try to overlap some stages of the computation with the processing of the upper levels. Unfortunately, the scheduler will only launch a new kernel when the resources are available, which typically happens towards the end of the BSR multiplication kernel. It therefore makes most sense to overlap the dense multiplication portion of the computation with the low rank portion. Many GPUs support a feature called stream priorities that allow execution of a kernel on a low priority stream to be suspended in favor of a kernel on a higher priority stream. By setting the dense phase as the lowest priority and the tree operations as the highest priority, I can effectively hide the overhead and hardware underuse of the tree operations as shown in Figure 4.3. The performance results below show the effect of

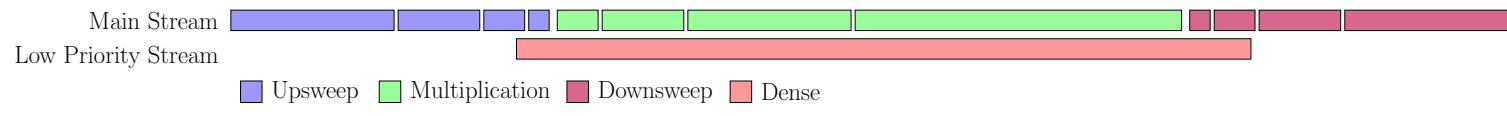

Figure 4.3: Overlapping the dense phase of the hgemv with the areas of low GPU utilization on a low priority stream. 
this overlap which, as expected, is beneficial for relatively small sized problems. On larger problems, the work at the higher levels of the trees is a very tiny fraction of the overall computation and there is relatively little benefit derived from the overlap. The $\widehat{x}$ and $\widehat{y}$ trees are stored in temporary workspace, allowing the dense and low rank phases to overlap, requiring only a single stream synchronization between the dense phase and the final phase of the downsweep.

\subsection{Performance Results}

To demonstrate the performance of the hgemv operation, I generate two families of hierarchical covariance matrices for a spatial Gaussian process with $n=2^{14} \cdots 2^{20}$ observation points placed on randomly perturbed $2 \mathrm{D}$ and $3 \mathrm{D}$ regular grids in $[0,1]^{2}$ and $[0,1]^{3}$ respectively. The covariance matrices are generated using an isotropic exponential kernel with correlation length 0.1 in 2D and 0.2 in 3D [66] and are representative of hierarchical matrices that arise in several applications [67].

The hierarchical matrices are generated $a b$ initio in the following way. The $n$ points are first partitioned into clusters using a KD-tree with a mean split, generating the index sets of the basis tree nodes. The basis and transfer matrices are then generated using Chebyshev interpolation [68]. A dual traversal [2, 69] of the basis tree generates the quadtree where the coupling matrices at the leaves are generated by evaluating the kernel at the interpolation points. The leaf size $m$ was set to 64 , tuned to the P100 GPU, and a uniform rank of 64 was used for all levels of the matrix, corresponding to the use of $8 \times 8$ grids and $4 \times 4 \times 4$ Chebyshev grids in $2 \mathrm{D}$ and 3D respectively. The choice of the leaf size only influences performance and has no effect on the accuracy of the representation, since overall accuracy is limited by the low rank blocks. The resulting approximation error is less than $10^{-7}$ in $2 \mathrm{D}$ and less than $10^{-3}$ in $3 \mathrm{D}$ for all problem sizes, as measured by computing $\left\|A x-A^{\mathcal{H}} x\right\| /\|A x\|$ where $A$ is the exact (dense) covariance, $A^{\mathcal{H}}$ is its hierarchical representation, and 

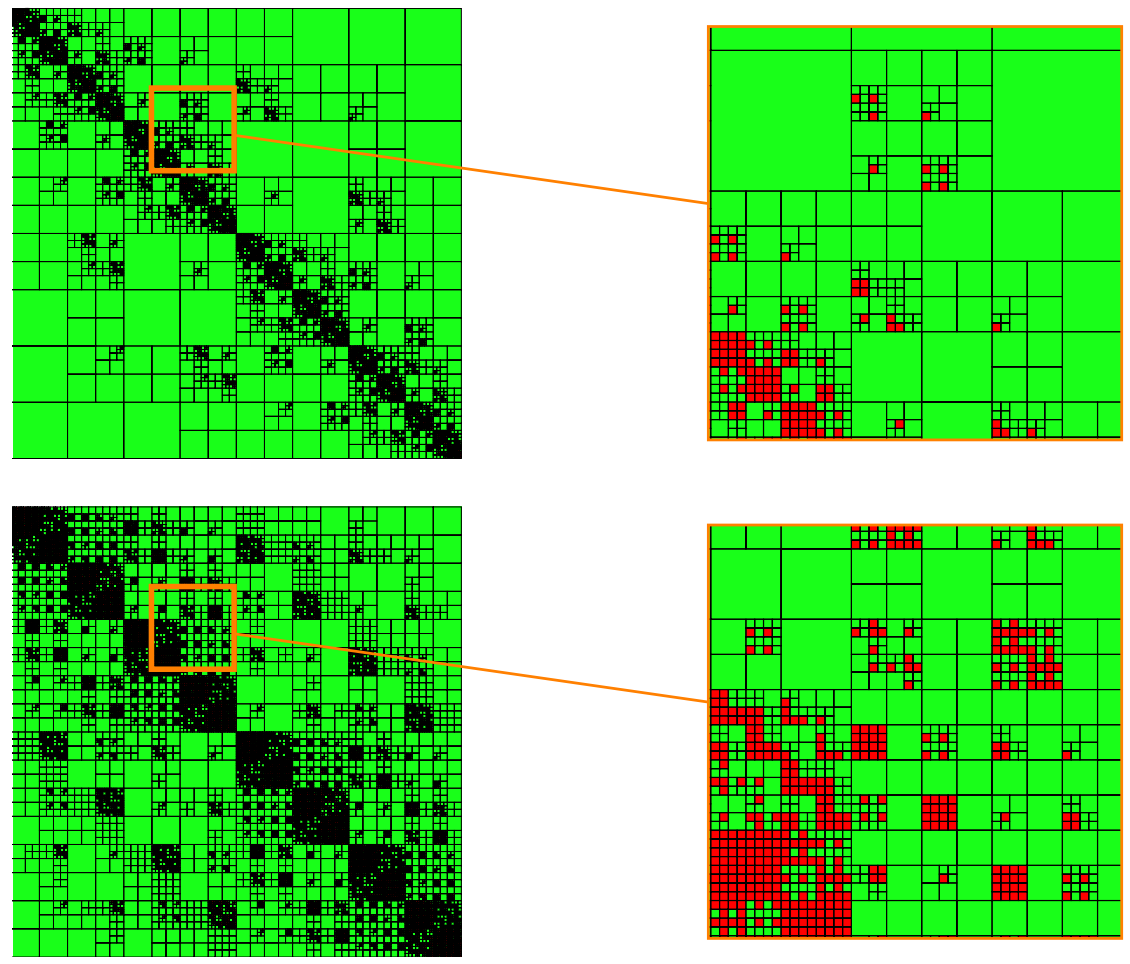

Figure 4.4: Structure of the 2D (top) and 3D (bottom) sample covariance matrices used in the examples for $n=2^{14}$, with a zoom on portions of them. Notice that the 3D problem does not have as many large blocks that admit a low rank approximation as the $2 \mathrm{D}$ problem and therefore results in a representation that has higher memory demand for the same accuracy. 
$x$ is a randomly generated vector whose entries are sampled from a uniform $[0,1]$ distribution. For the large problems, it is not possible to store the dense $A$ nor is it practical to perform the $\mathcal{O}\left(n^{2}\right) A x$ product, and as a result I sampled $10 \%$ of the rows and used the analytical expression of the matrix entries. While a rank of 64 may seem high for a leaf size of 64 , it is often the case that the ranks at the leaves increase temporarily due to low rank updates that may be applied to the blocks of the matrix during hierarchical matrix operations. This rank will be reduced during compression and the effect on matrix vector multiplication performance is shown in Section 5.5.
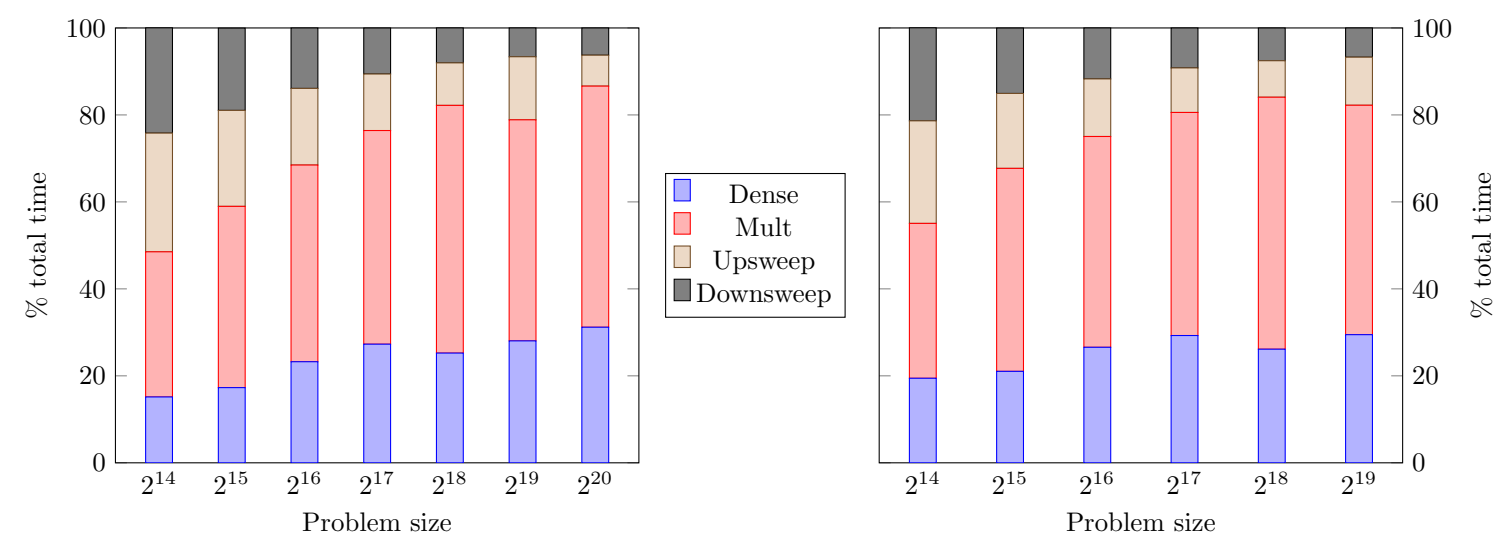

Figure 4.5: Breakdown of the hgemv phases in percentages of total operation time in single (left) and double (right) precision on a P100 GPU showing that, for smaller problem sizes where the basis tree has very few levels, the upsweep and downsweep do not fully utilize the hardware.

For illustration, Figure 4.4 shows the structure of the 2D and 3D covariance matrices for the $n=2^{14}$ problem size. The small dense blocks of size $64 \times 64$ are shown in red in the zoomed details. As expected from physical intuition about distance-based interaction strength in higher dimensions, the 3D matrix has more dense blocks and its low rank trees are bushier than those of the 2D matrix which puts more pressure on memory (or alternatively permit lower accuracy for the same memory footprint as a $2 \mathrm{D}$ problem of the same size). The performance results shown here are for these matrices in their original analytically derived hierarchical form. The algebraic com- 


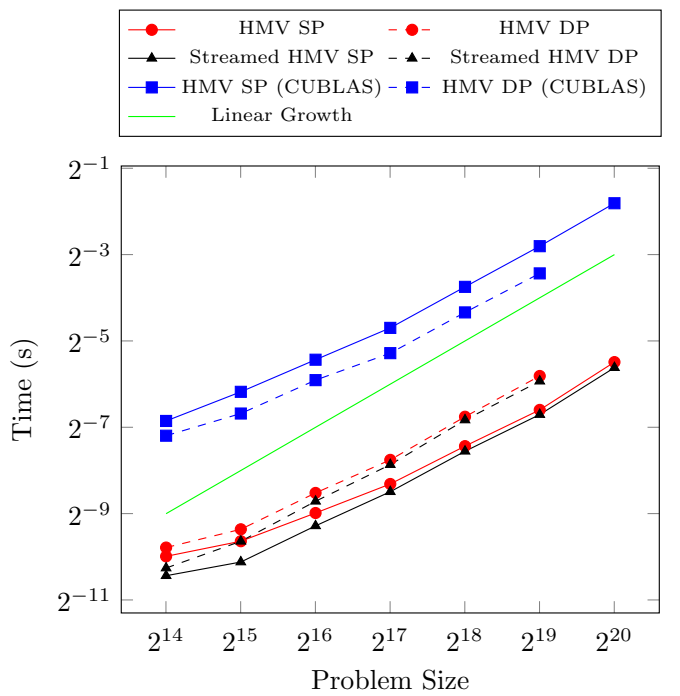

(a) Time for the hgemv in single and double precision for a $2 \mathrm{D}$ problem.

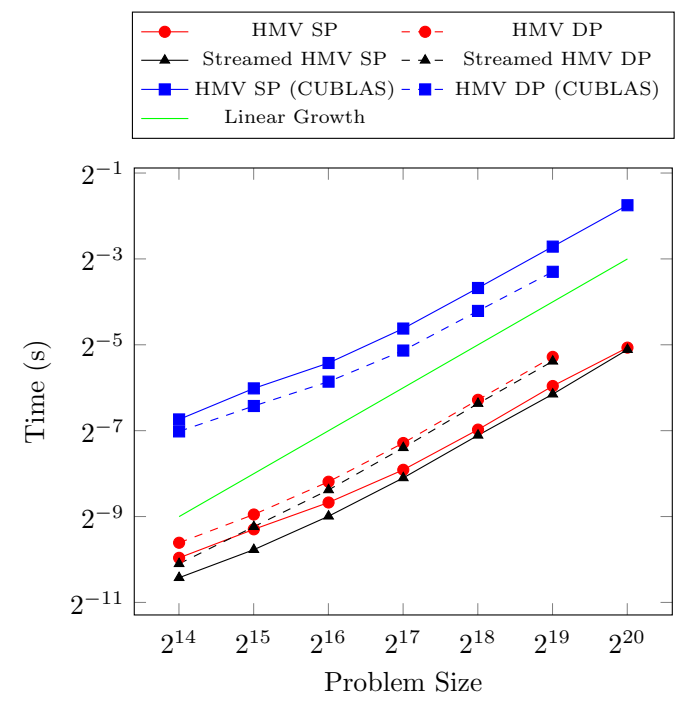

(b) Time for the hgemv in single and double precision for a $3 \mathrm{D}$ problem.

Figure 4.6: Runtime of hgemv on a single P100 GPU, showing asymptotically linear growth with problem size. Notice that the streamed version that allows overlapping between the dense and the low rank phases of hgemv provides performance boost on small problems. On the larger problems, where the available bandwidth is saturated with the low rank data, the improvement due to overlapping is diminished.

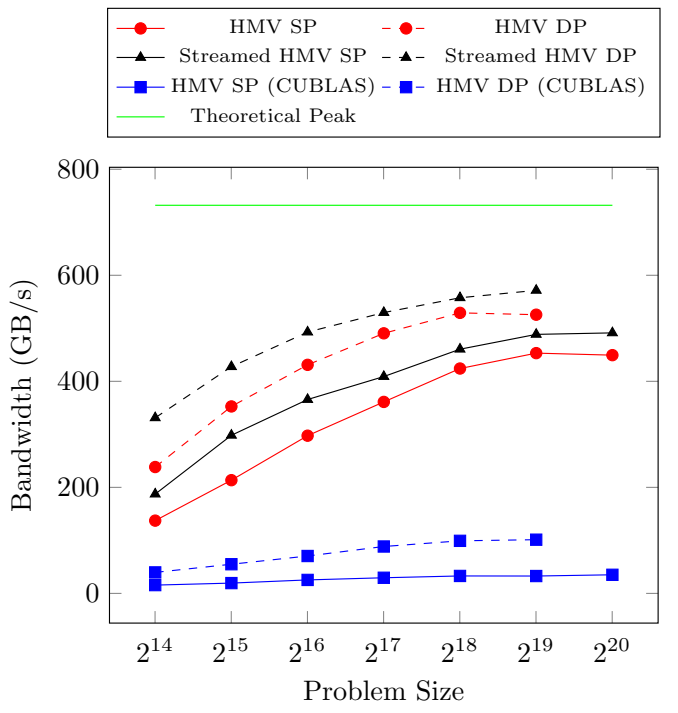

(a) Achieved bandwidth for the hgemv in single and double precision for a $2 \mathrm{D}$ problem.
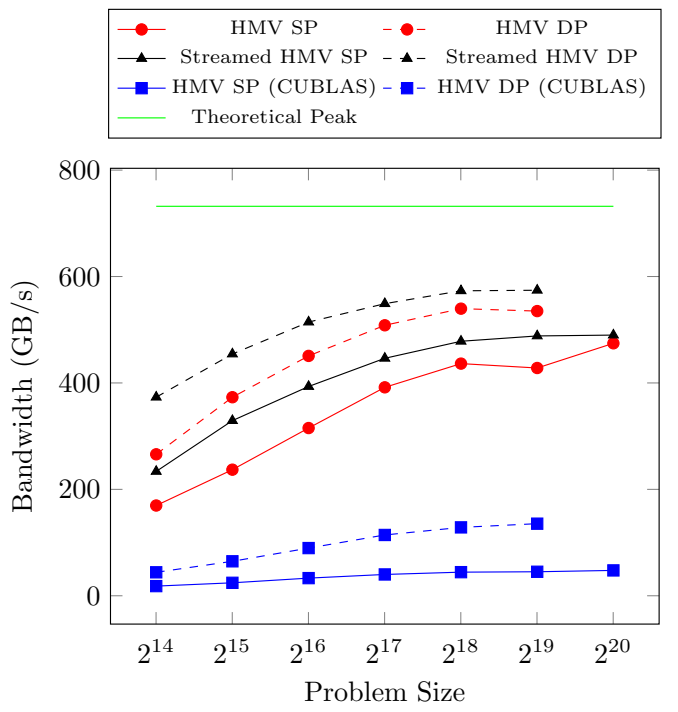

(b) Achieved bandwidth for the hgemv in single and double precision for a 3D problem.

Figure 4.7: Achieved bandwidth of hgemv on a single P100 GPU with the streamed kernel achieving up to $78 \%$ of the theoretical bandwidth peak of the GPU. 
pression discussed in Chapter 5 reduces the memory footprint of the low rank portions substantially and further reduce the hgemv time, as shown in Section 5.5.

Figure 4.5 shows the profile of the execution of the hgemv of the $3 \mathrm{D}$ problems in single and double precision. The upsweep and downsweep phases show relatively poor performance for the smaller problems sizes, since the small batches generated for the upper levels of the trees do not provide enough work to saturate the GPU, leading to lower performance during those phases. The impact of the smaller higher levels is alleviated as the problem size increases, where the larger lower levels that can saturate the GPU dominate the workload. Kernel streaming as discussed in Section 4.4 allows increased hardware usage during those phases.

Figures $4.6 \mathrm{a}$ and $4.6 \mathrm{~b}$ show the execution time of the hgemv in double and single precision for the 2D and 3D problems respectively. The streamed kernel shows up to $27 \%$ performance improvement for the smaller problem sizes. We can also see the expected linear growth of the algorithm for all versions. I also compare the effect of using different batched kernels. All versions using the batched GEMV and block spMV kernels from [70, 71] show significant improvements over the same algorithms implemented with the vendor provided routines in cuBLAS [47] and cuSPARSE [72]. Since the matrix vector multiplication is a memory-bound routine, I gauge the performance of the kernels by bandwidth.

Since this operation is bandwidth limited, I compute performance as the total number of bytes transferred over total execution time. This includes all dense and coupling matrices in the matrix tree as well as the leaves and transfer matrices of the basis trees. Figures $4.7 \mathrm{a}$ and $4.7 \mathrm{~b}$ show the achieved bandwidth of the various hgemv kernels, with the streamed version achieving up to $78 \%$ of the theoretical peak bandwidth of the P100 GPU. The improvement in achieved bandwidth over the non-streamed version that does not allow the overlap of the different portions of the computation is substantial for the small problem sizes. 


\section{Chapter 5}

\section{Orthogonalization and Compression}

Compression is a core operation in hierarchical matrix algebra. For example, in the course of implementing BLAS3 operations, matrix blocks get added, generally producing increased apparent ranks. The matrix needs to be re-compressed in order to maintain the optimal complexity. The goal of compression is therefore to construct new nested row and columns bases in which the matrix blocks can be expressed more compactly, i.e., where blocks originally represented as $U_{i} S_{i j} V_{j}^{T}$ can be compressed into the form $U_{i}^{\prime} S_{i j}^{\prime} V_{j}^{\prime T}$ where the dimensions of $S^{\prime}$ are smaller than the dimensions of $S$. The primary task here is to construct the common basis $U_{i}^{\prime}$ in which all blocks of a given block row can be expressed, without incurring the quadratic cost that would be needed if a straight SVD of the whole row block is performed. The same goes for column blocks and $V_{j}^{\prime}$ when the two bases $\mathcal{U}$ and $\mathcal{V}$ are different. Finally, once the compact and more efficient nested block row/column basis has been generated, the new $S_{i j}^{\prime}$ for every matrix block is computed by a transformation of the form $T_{U i} S_{i j} T_{V j}^{T}$, leading to a smaller memory footprint and reduced hierarchical operation runtimes. The algorithms presented are adapted from [73] to fit the architecture of the GPU.

\subsection{Overview}

In order to introduce the somewhat complicated algorithm, let us first consider how the new basis for a block row $A_{i}^{q}$ at the finest level $q$ would be generated. $A$ here denotes only the low rank portions of the hierarchical matrix, since the dense blocks 
are not compressed. $A_{i}^{q}$ consists of $b$ low rank blocks expressed at level $q$ as $U_{i} S_{i j} V_{j}^{T}$ with $j=j_{1} \cdots j_{b}$, and additional pieces representing the restriction of blocks from higher levels to their " $t$ " rows as shown in Figure 5.1 .

$$
A_{i}^{q}=U_{i}^{q}\left[\begin{array}{c}
\text { portions of } \\
\text { ancestors }
\end{array} S_{i j_{1}}^{q} V_{j_{1}}^{q T} \cdots S_{i j_{b}}^{q} V_{j_{b}}^{q T}\right]=U_{i}^{q} B_{i}^{q T}
$$

The optimal basis can be generated by computing the SVD of $U_{i}^{q} B_{i}^{q T}$, truncating it to the desired approximation, and using the truncated left singular vectors as the new basis $U_{i}^{\prime q}$. This would however require the expensive SVD of the $O(n)$-sized $B_{i}^{q}$. In order to avoid it, I first compute the QR decomposition of $B_{i}^{q}$ and then perform the SVD with the small $R$ factor.

$$
A_{i}^{q}=U_{i}^{q} B_{i}^{q T}=U_{i}^{q}\left(Q_{i}^{q} R_{i}^{q}\right)^{T}=\underbrace{U_{i}^{q} R_{i}^{q T}}_{\text {new basis }} Q_{i}^{q T}
$$

The optimal basis $U_{i}^{\prime q}$ is then simply the truncated left singular vectors of what might be thought of as a new weighted basis $U_{i}^{q} R_{i}^{q T}$, and this finishes the process for level $q$. When moving to higher levels in the tree, I need to insure that the $U^{\prime}$ bases remain nested. This requires additional singular value decompositions, but involving only small transfer matrices, as I go up $\mathcal{U}$ in an upsweep traversal described in Section 5.4.

The task of computing $R_{i}^{q}$ of the QR decomposition of $B_{i}^{q}$ can be done efficiently by exploiting the nestedness of the bases. Let us assume that the QR decomposition of $B_{i^{+}}^{q-1}$, the parent block $i^{+}$at level $q-1$, is available as $Q_{i^{+}}^{q-1} R_{i^{+}}^{q-1}$. Then,

$$
\begin{aligned}
& A_{i}^{q}=\left[\begin{array}{l}
i \text {-portion of } \\
U_{i^{+}}^{q-1} B_{i^{+}}^{q-1} T
\end{array} \quad U_{i}^{q} S_{i j_{1}}^{q} V_{j_{1}}^{q T} \cdots U_{i}^{q} S_{i j_{b}}^{q} V_{j_{b}}^{q T}\right] \\
& =U_{i}^{q}\left[E_{i}^{q}\left(Q_{i^{+}}^{q-1} R_{i^{+}}^{q-1}\right)^{T} \quad S_{i j_{1}}^{q} V_{j_{1}}^{q T} \cdots S_{i j_{b}}^{q} V_{j_{b}}^{q T}\right]=U_{i}^{q} B_{i}^{q T}
\end{aligned}
$$




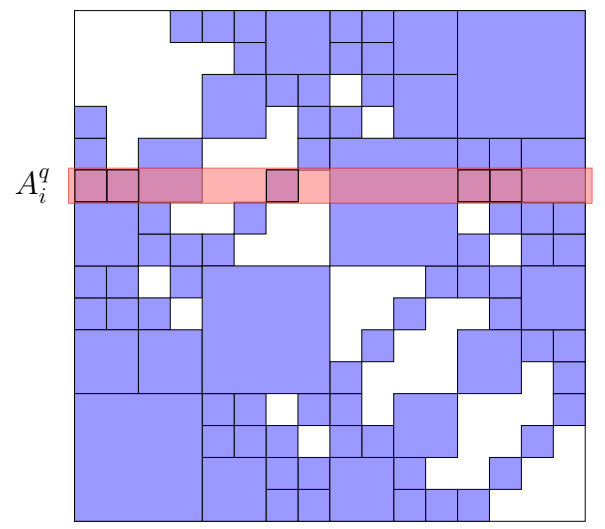

Figure 5.1: Matrix block row $A_{i}^{q}$ of the low rank portion of the hierarchical matrix for the sixth row of the leaf level. $A_{i}^{q}$ includes 5 blocks at level $q$ and sub-blocks coming from higher level block rows.

with $B_{i}^{q}$ conveniently expressible as:

$$
B_{i}^{q}=\left[\begin{array}{c}
Q_{i^{+}}^{q-1} R_{i^{+}}^{q-1} E_{i}^{q T} \\
V_{j_{1}}^{l} S_{i j_{1}}^{q T} \\
\vdots \\
V_{j_{b}}^{q} S_{i j_{b}}^{q T}
\end{array}\right]=\operatorname{diag}\left(Q_{i^{+}}^{q-1}, V_{j_{1}}^{q}, \cdots, V_{j_{b}}^{q}\right)\left[\begin{array}{c}
R_{i^{+}}^{q-1} E_{i}^{q T} \\
S_{i j_{1}}^{q T} \\
\vdots \\
S_{i j_{b}}^{q T}
\end{array}\right]
$$

Assuming the $V^{q}$ bases are orthogonal, the block diagonal matrix in Eq. 5.4 is orthogonal and the QR of $B_{i}^{q}$ simply reduces to the QR of the small stack at the end of Eq. 5.4 which involves only $b+1$ blocks, each being a small $k \times k$ coupling/transfer matrix, and therefore can be done quite efficiently. Since this QR uses the $R^{q-1}$ matrix from level $q-1$, the overall computation starts from the root and goes down the tree computing all the $R_{i}^{l}$ matrices for all levels in a downsweep traversal. As with previous operations, all blocks at a given level can obviously be processed in parallel. I also observe here that the $Q$ factors are not needed and a specialized QR decomposition avoiding their expensive storage and computation improves the performance of the algorithm.

Orthogonalizing the $\mathcal{V}$ basis tree can be done in a pre-processing phase. A basis 
is orthogonal if $V_{j}^{l T} V_{j}^{l}$ is the identity matrix for all levels $l$. Orthogonalizing a basis involves performing $\mathrm{QR}$ on the finest level basis and then going up the tree to compute new transfer matrices that allow higher level nodes to satisfy the orthogonality condition. This is also done via additional QR operations involving the transfer matrices.

In summary, the overall compression procedure consists of the following three computational steps:

- An upsweep of the basis trees to orthogonalize them. This step uses a sequence of batched QR calls, one per level, to produce an orthogonal basis. This is described in Section 5.2.

- A downsweep of the basis trees, using the coupling blocks $S_{i j}$, to construct the $R_{i}$ factors for the new bases. This step uses a sequence of batched QR kernel calls, one per level, on the stacks at the end of Eq. 5.4. This is described in Section 5.3 .

- An upsweep of the basis tree to truncate the new bases to the desired tolerance. This step uses a sequence of batched SVD calls, again one per level, on the $U_{i} R_{i}$ bases to produce the new optimal basis. The $S_{i j}$ blocks are then transformed into these bases via batched matrix multiplication operations. This is described in Section 5.4.

\subsection{Basis Orthogonalization}

Orthogonalizing a nested basis tree $\mathcal{V}$ replaces it by a new nested basis where every node satisfies the orthogonality condition $V_{j}^{l T} V_{j}^{l}=I$. This is equivalent to the conditions that $V_{j}^{q T} V_{j}^{q}=I$ at the finest level $q$ and that the transfer matrices satisfy $\sum_{c} F_{c}^{T} F_{c}=I$ for all levels, where $c$ ranges over the two children of every node in the basis tree. Besides allowing the simplification in the $R$ computation algorithm, 
orthogonalizing the basis of an $\mathcal{H}$-matrix simplifies error computations and matrix projections. It is also structurally similar to the truncation algorithms described in Section 5.4.

A by-product of orthogonalization is also a set of projection matrices that transform between the old basis and the new orthogonal one. These projection matrices are stored in a tree that shares the same structure as the basis tree, where processing each node of the basis tree produces a node in the projection tree $T_{\mathcal{V}}$ I assume here that $\mathcal{V}=\mathcal{U}$ and do not make a distinction between the row and column bases and drop the subscript $\mathcal{V}$ from $T$. By denoting $Q_{i}^{l}$ the new orthogonal basis at level $l$, the original basis $V_{j}^{l}$ can be recovered as $Q_{j}^{l} T_{j}^{l}$. I will use this transformation to express the coupling matrices in the new orthogonal basis.

As in the upsweep of the hgemv algorithm, I perform the orthogonalization operation one level at a time, starting at the leaves and sweeping up the tree. Processing the leaves simply requires $\mathrm{QR}$ factorization of each leaf node where the $Q$ factor becomes the new orthogonal leaf and the $R$ factor is output into the leaf level of $T$. The inner nodes are expressed in terms of their children using the nested basis property and must be orthogonalized in a way that preserves this property. Given an inner node $j^{+}$at level $l-1$ with children $j_{1}$ and $j_{2}$ at processed level $l$, we have

$$
V_{j^{+}}^{l-1}=\left[\begin{array}{cc}
V_{j_{1}}^{l} & \\
& V_{j_{2}}^{l}
\end{array}\right]\left[\begin{array}{l}
F_{j_{1}} \\
F_{j_{2}}
\end{array}\right]=\left[\begin{array}{ll}
Q_{j_{1}}^{l} & \\
& Q_{j_{2}}^{l}
\end{array}\right]\left[\begin{array}{l}
T_{j_{1}}^{l} F_{j_{1}} \\
T_{j_{2}}^{l} F_{j_{2}}
\end{array}\right]=\left[\begin{array}{ll}
Q_{j_{1}}^{l} & \\
& Q_{j_{2}}^{l}
\end{array}\right] Z
$$

Forming the $2 k^{l} \times k^{l-1}$ matrix $Z$, computing its QR factorization and using the two $k^{l} \times k^{l-1}$ blocks of the $Q$ factor as the new transfer matrices, gives us the new orthogonal inner nodes that satisfies the nested basis property. The $R$ factor is then output into level $l-1$ of the projection tree $T$. $Z$ is formed by first marshaling the operations based on the data in the projection tree and the basis tree transfer nodes and then operating on them using a batched matrix-matrix multiplication routine. 


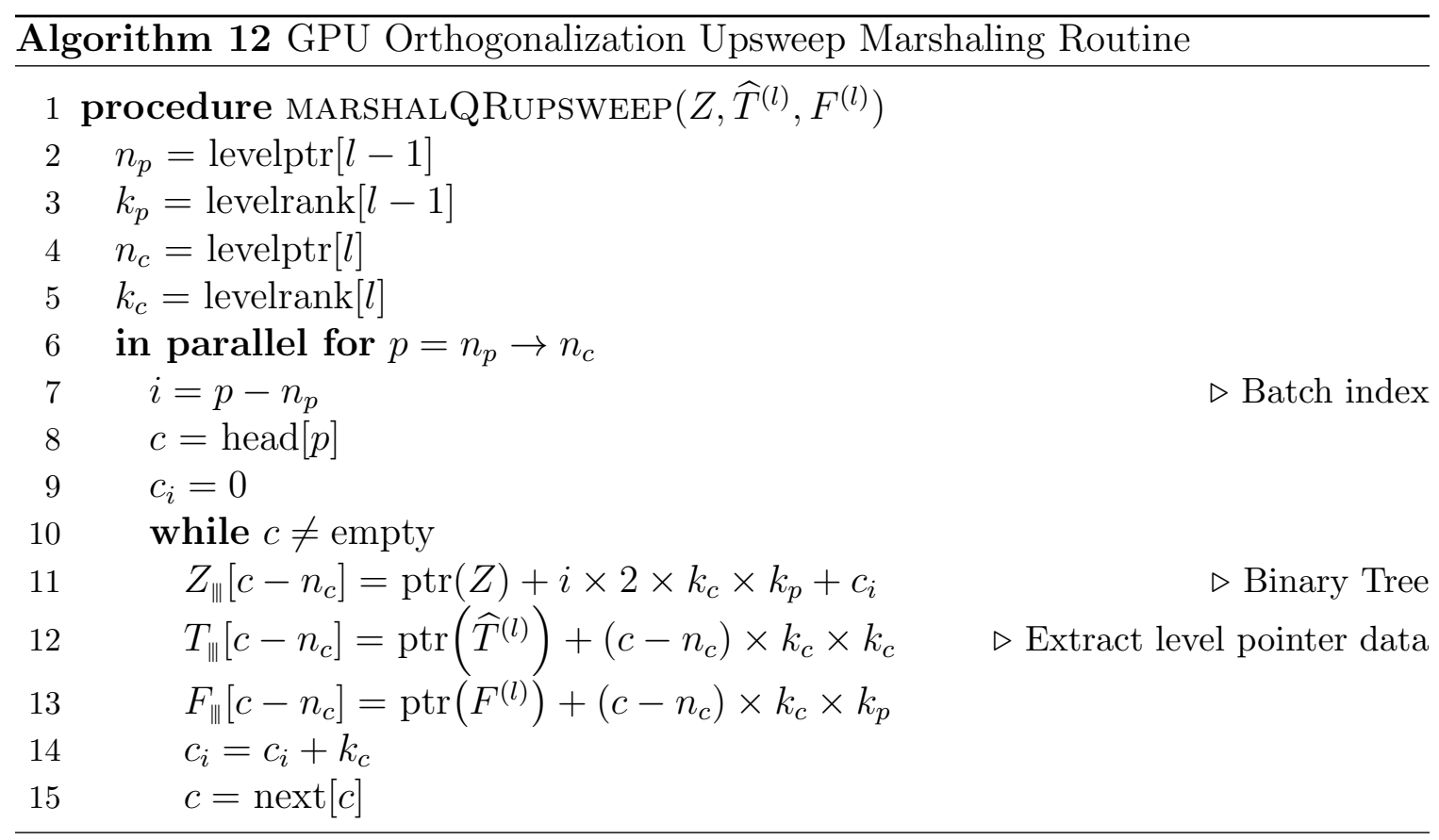

$Z$ is then factorized using a batch QR factorization routine and the sub-blocks are copied back as the new transfer matrices using a marshaled batch copy operation. This leads to Algorithm 12 for marshaling the orthogonalization upsweep operations and Algorithm 13 for computing the projection tree $T$ and the new orthogonal basis. The left side of Figure 5.2 depicts this operation for the binary basis tree.

Finally, the projection phase transforms the coupling matrices of each matrix

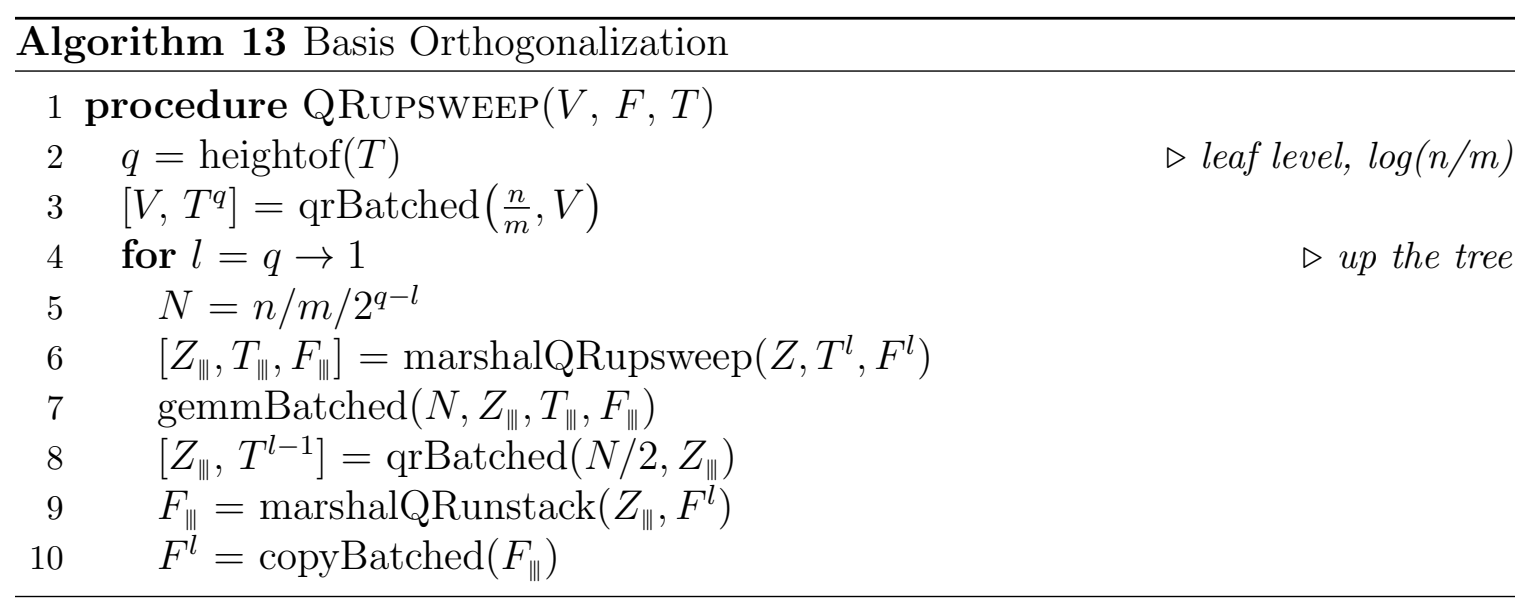



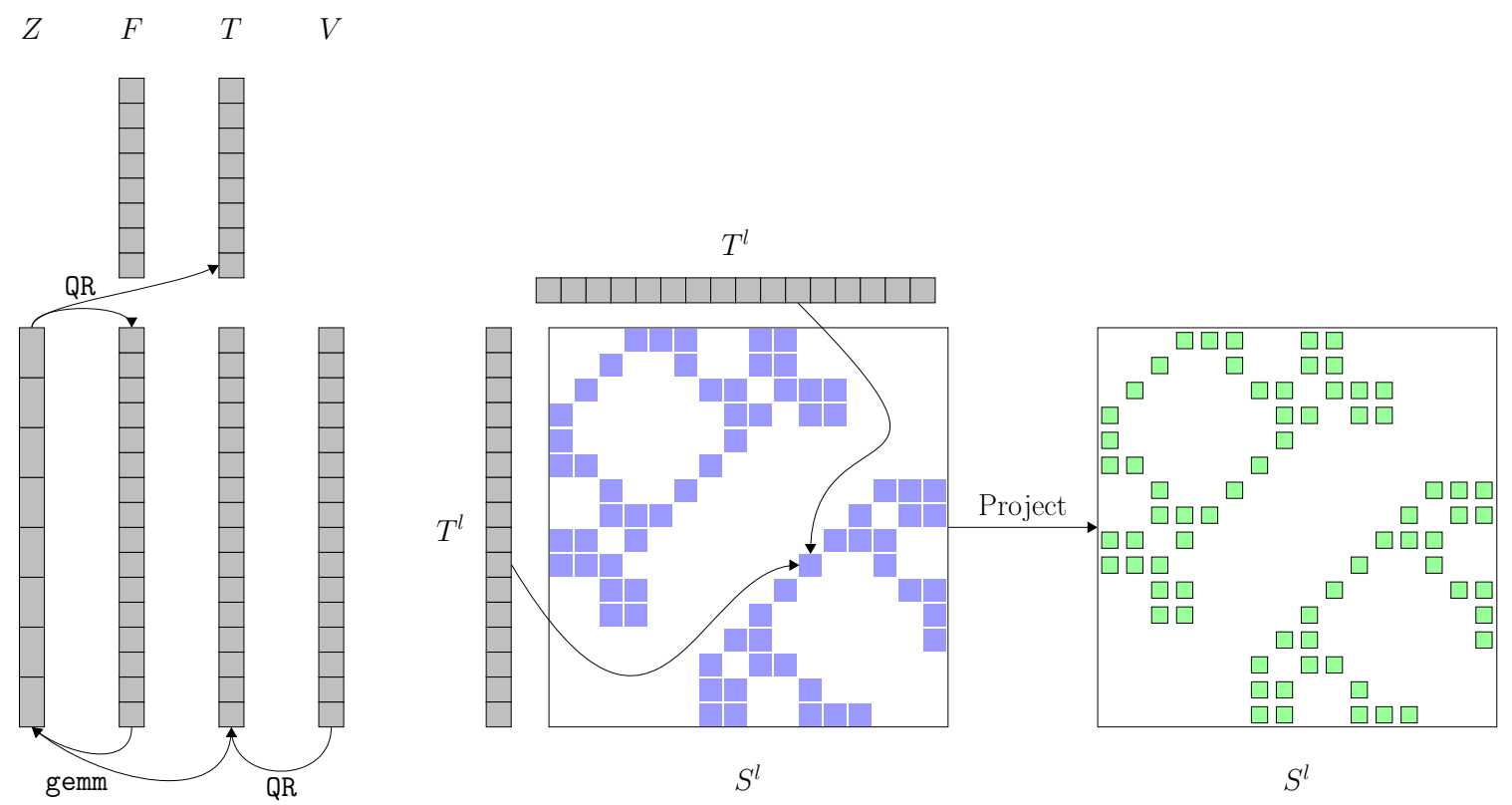

Figure 5.2: Left: Basis orthogonalization starting from the leaves and sweeping up the tree to overwrite the basis with an orthogonal one and generate the projection tree $T$ (only a single level is depicted here). Right: Projection of the leaf level of coupling matrices into a new basis using a projection tree $T$. The new basis could be more compact, resulting in lower rank for the level.

block $A_{i j}^{l}$ at level $l$ using the projection matrices stored in $T^{l}$ :

$$
A_{i j}^{l}=U_{i}^{l} S_{i j}^{l} V_{j}^{l T}=Q_{i}^{l}\left(T_{i}^{l} S_{i j}^{l} T_{j}^{l T}\right) Q_{j}^{l T}
$$

The new coupling matrices are obtained by left and right multiplications with the computed projection matrices independently at all levels. The operations are first marshaled by level and then executed using batch matrix-matrix multiplication routines. This operation is described in Algorithm 14 and depicted in the right panel of Figure 5.2 for a single level of the matrix tree.

\subsection{Basis Generation}

In this phase, a basis tree $R$ for the block rows of the matrix tree is constructed. This tree will have the same structure as the row basis tree $U$. Every node $i$ at every level 


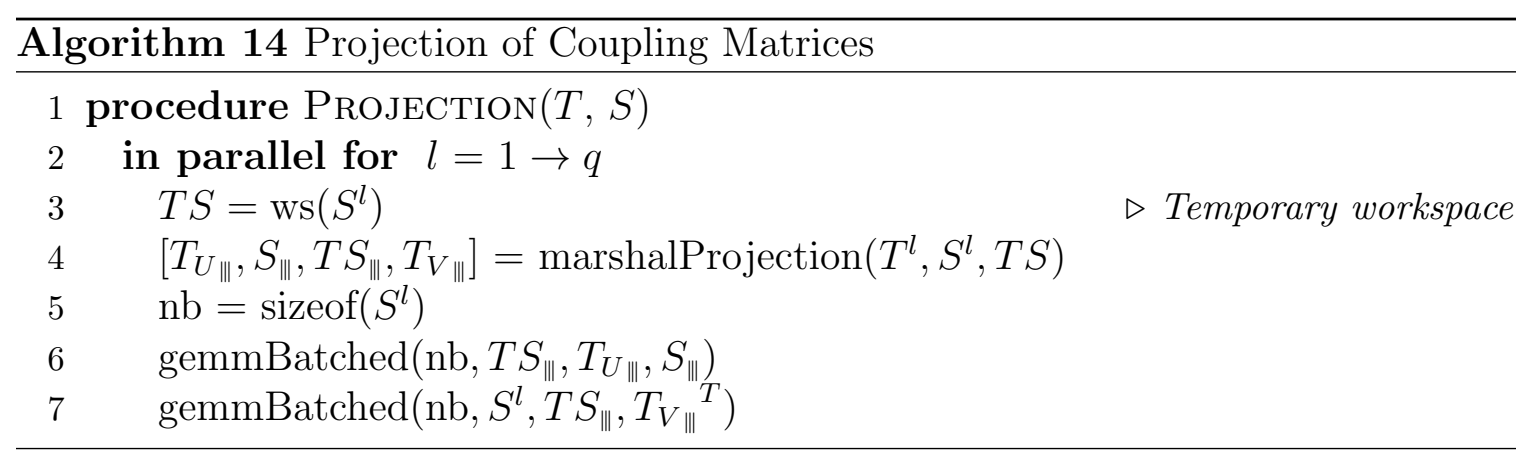

$l$ will store the matrix $R_{i}^{l}$ which will postmultiply the corresponding $U_{i}^{l}$ to produce the new basis that will be truncated. As described earlier, $R_{i}^{l}$ depends on matrix data coming from bocks at level $l$ whose row basis node is $U_{i}$ and from higher level blocks that also span the block row $i$.

Denoting the parent node of $i$ by $i^{+}$, the relevant block row $\bar{S}_{i}$ that enters the computation of $R_{i}^{l}$ is depicted in Figure 5.3 and expressed as:

$$
\bar{S}_{i}=\left[\begin{array}{c}
R_{i^{+}}^{l-1} E_{i}^{l T} \\
S_{i j_{1}}^{l T} \\
S_{i j_{2}}^{l T} \\
\vdots \\
S_{i j_{b}}^{l T}
\end{array}\right]
$$

where $b$ is the number of blocks in the block row at level $l$. The first block $R_{i^{+}}^{l-1} E_{i}^{l T}$ represents data coming from the levels of blocks above $l$. The node $R_{i}^{l}$ can then be computed as the $R$ factor of the QR factorization of $\bar{S}_{i}$. The tree is computed starting at the root of the matrix tree followed by a sweep down to the leaves. Marshaling the block row from the matrix tree data and the parent level of $R$ into a batch matrixmatrix multiplication and a batch transpose operation allows the quick formation of $\bar{S}_{i}$ for a level. This matrix is then fed into a batch QR factorization routine that does not form $Q$. The marshaling routine for this operation makes use of the generated BSR data of the level of the matrix tree and is described in Algorithm 16. Putting 


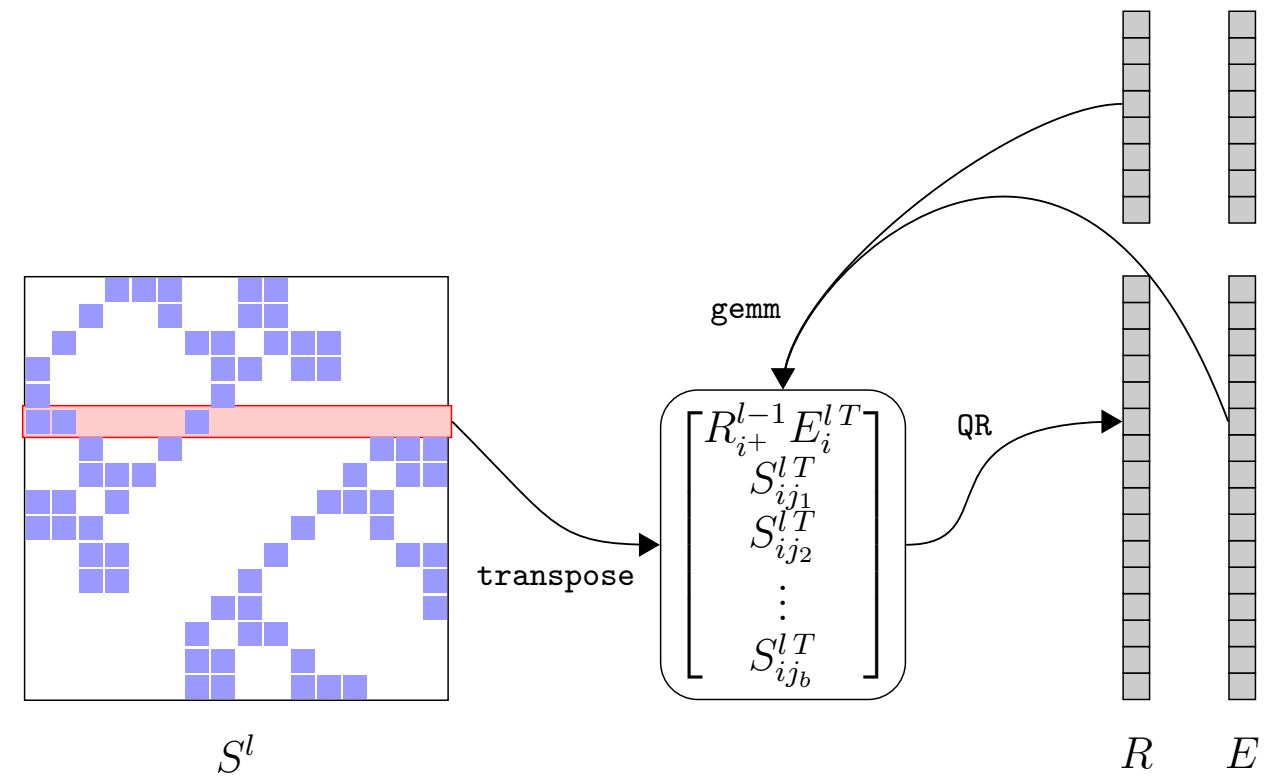

Figure 5.3: Constructing $R_{i}^{l}$ from its parent level, the matrix tree row data, and transfer matrices.

everything together leads to Algorithm 15 to form the tree $R$.

Once the $R$ matrix of each block row is computed, I can generate the new compressed basis tree, which allows the ranks of the blocks at every level $l$ to decrease from $k^{l}$ to $\widetilde{k}^{l}$ while maintaining a prescribed accuracy $\epsilon$ in the approximation. This is the heart of algebraic compression.

The truncation process is structurally similar to the upsweep of the orthogonalization in that processing the nodes produces projection matrices which are then

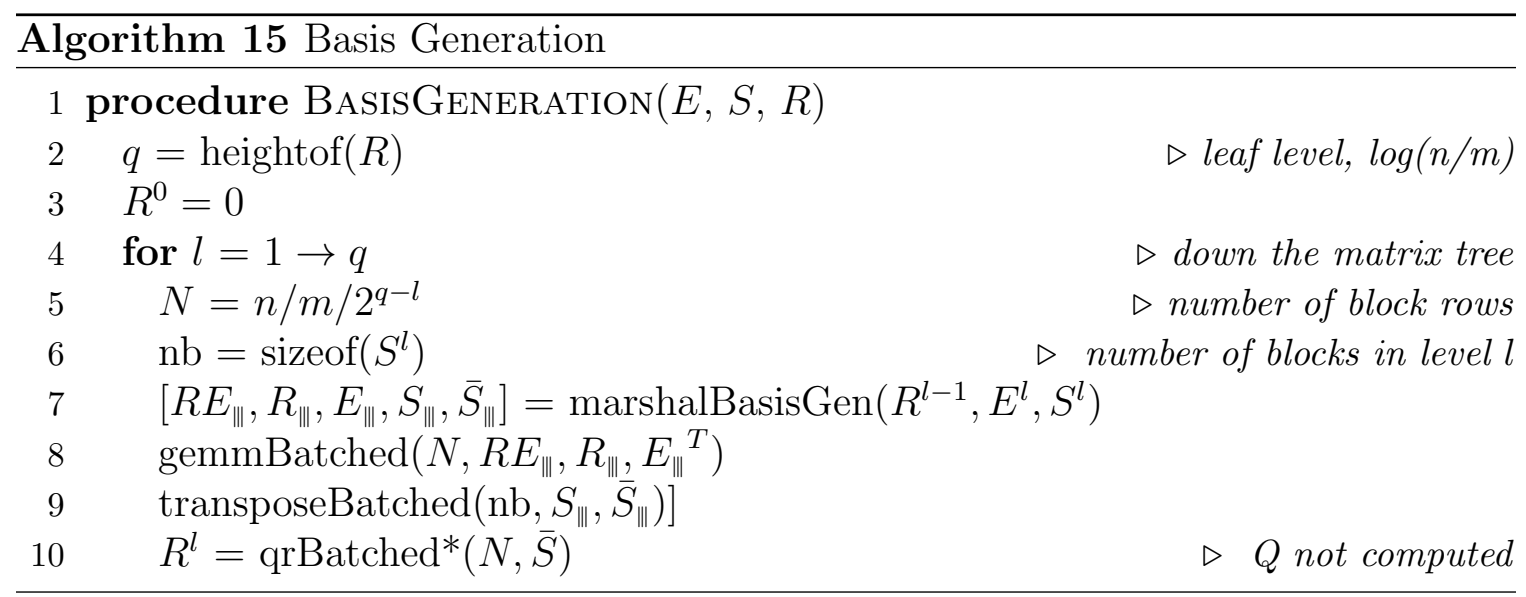




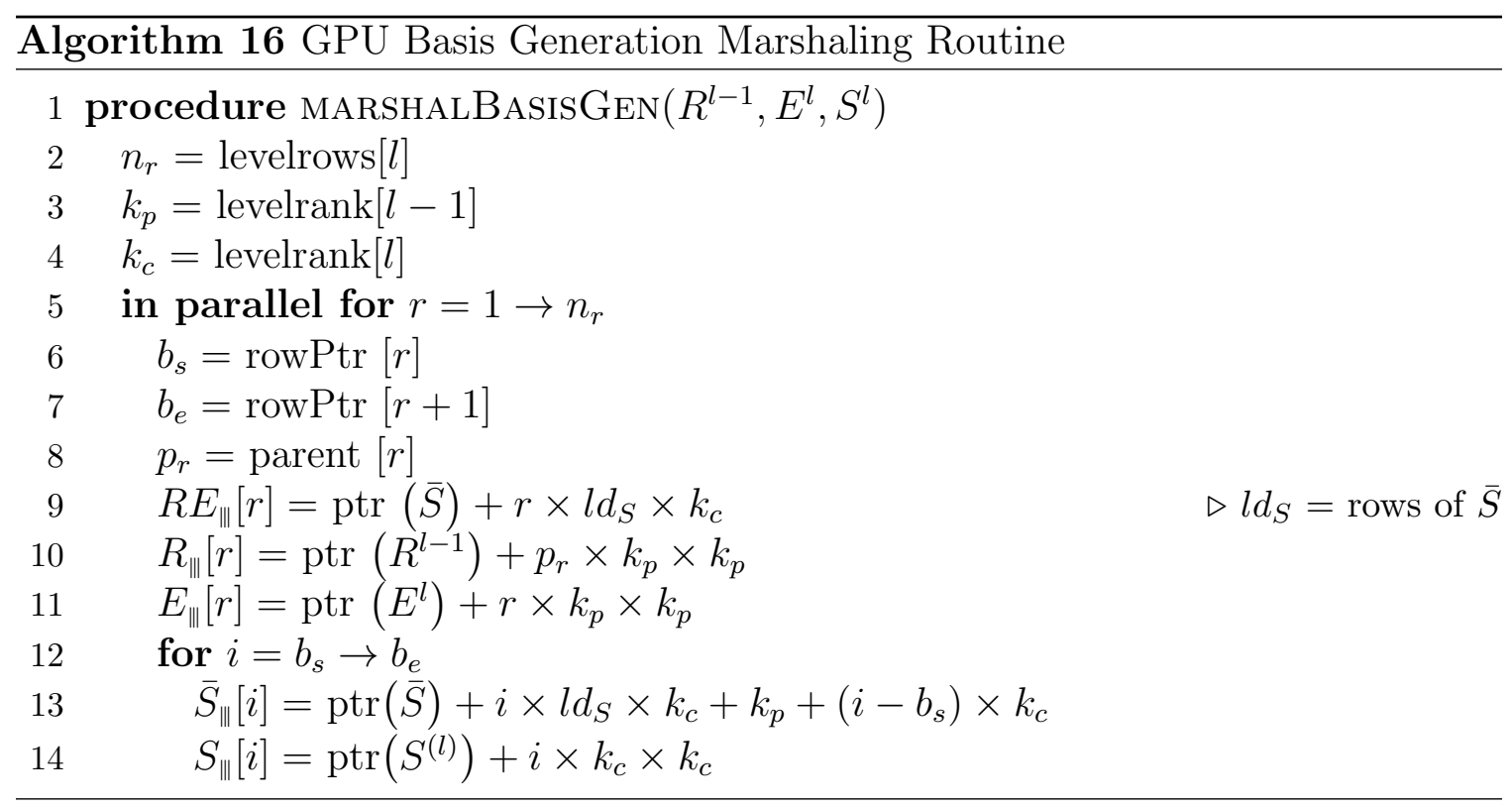

used to sweep up the tree; however processing each node involves different computational kernels. For the leaf nodes $U_{i}^{q}$, I first use $R_{i}^{q}$ to produce a new basis node $W_{i}^{q}=U_{i}^{q} R_{i}^{q T}$. I then compute the singular value decomposition of $W_{i}^{q}$ and produce the truncated basis by discarding singular vectors corresponding to values that are less than a threshold relative to the largest singular value.

As I impose a constant rank per level, a fast reduction computes the maximum truncated rank $\widetilde{k}^{q}$ for the given tolerance at the leaf level. The truncated left singular vectors $Q_{i}^{q}$ will be the new compact basis node. Finally, the projection matrix into the new basis is computed as $T_{i}^{q}=Q_{i}^{q T} U_{i}^{q}$.

\subsection{Basis Truncation}

Processing the inner nodes of the tree follows the same procedure as the orthogonalization: I form the $2 \widetilde{k}^{l} \times k^{l-1}$ matrix $Z$ using the original transfer matrices and the projection matrices. I then compute a weighted $Z$ matrix, $W_{i}^{l}=Z_{i} R_{i}^{l}$, and proceed to compute its truncated singular value decomposition. The two $\widetilde{k}^{l} \times \widetilde{k}^{l-1}$ blocks of the truncated left singular vectors $Q_{i}^{l}$ will be the new transfer matrices for the truncated inner node, and the projection matrix is computed as $T_{i}^{l}=Q_{i}^{l T} Z_{i}$. The marshaling 


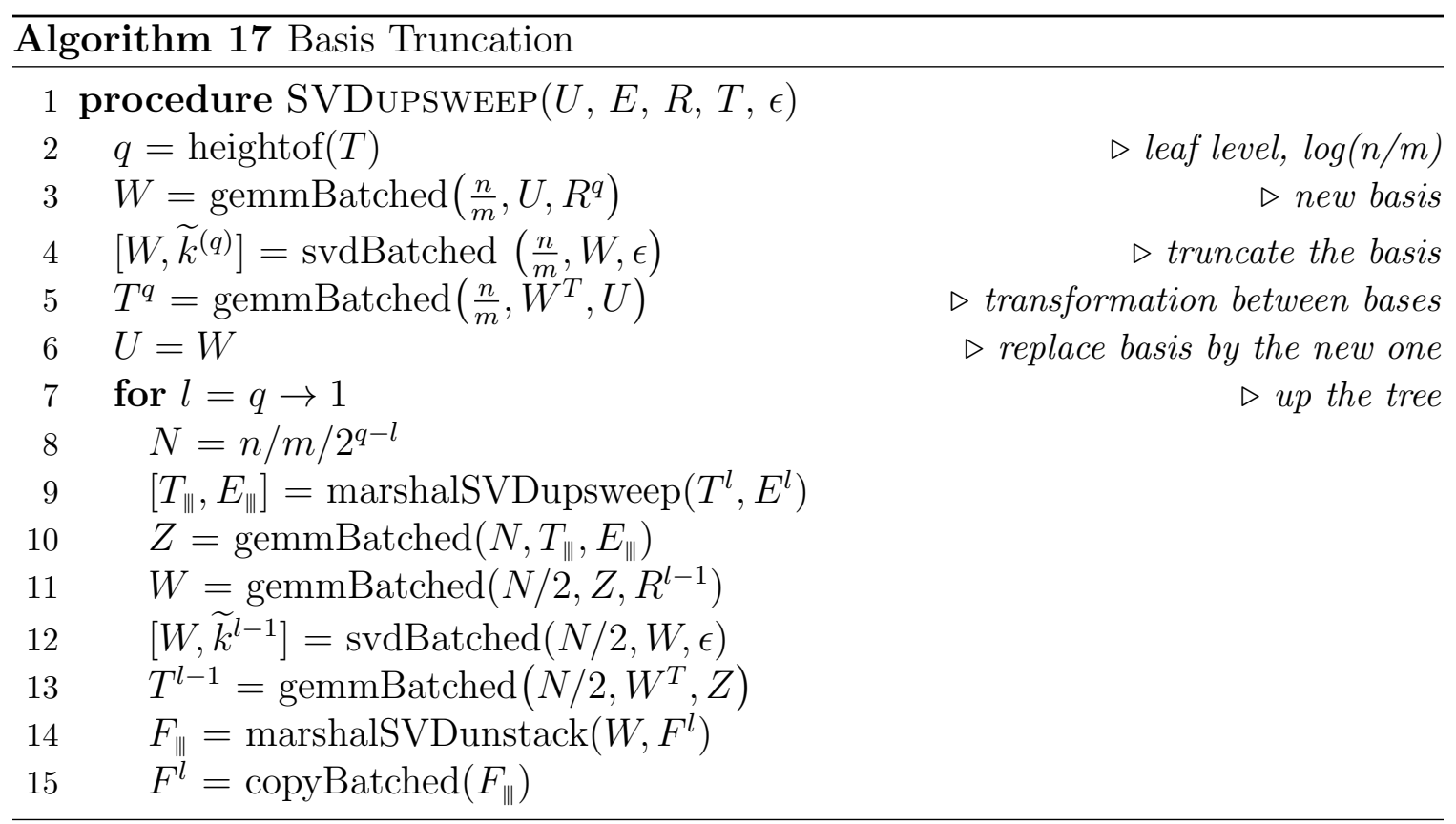

procedures are very similar to those of the orthogonalization with the addition of applying the factor $R$ to the original basis. The operations are then carried out by batch singular value decompositions and matrix-matrix multiplications. This leads to Algorithm 17 for producing the new compact basis and the corresponding projection matrix tree $T$ given a relative threshold $\epsilon$ for truncation.

Finally, the projection of the coupling matrices is carried out in the same way as the projection phase of the orthogonalization procedure, using marshaled batch matrix-matrix multiplications with the projection matrix tree produced by Algorithm 17.

\subsection{Performance}

I study the performance of the GPU compression procedure using the same two families of 2D and 3D covariance matrices described in Section 4.5. The matrices were originally generated as hierarchical matrices in a generic polynomial basis. As a result, their representation is not particularly memory efficient and algebraic compression can be therefore expected to produce new compressed hierarchical representation to 
91

\begin{tabular}{|c|c|c|c|}
\hline Problem size & $\frac{\left\|A_{2}^{\mathcal{H}}-A_{1}^{\mathcal{H}}\right\|_{F}}{\left\|A_{1}^{\mathcal{H}}\right\|_{F}}$ & $\frac{\left\|A x-A_{1}^{\mathcal{H}} x\right\|}{\|A x\|}$ & $\frac{\left\|A x-A_{2}^{\mathcal{H}} x\right\|}{\|A x\|}$ \\
\hline \hline $2^{14}$ & $6.40 \times 10^{-8}$ & $3.33 \times 10^{-7}$ & $3.12 \times 10^{-7}$ \\
$2^{15}$ & $9.85 \times 10^{-8}$ & $3.60 \times 10^{-7}$ & $3.36 \times 10^{-7}$ \\
$2^{16}$ & $1.14 \times 10^{-7}$ & $3.47 \times 10^{-7}$ & $3.50 \times 10^{-7}$ \\
$2^{17}$ & $1.52 \times 10^{-7}$ & $3.50 \times 10^{-7}$ & $3.58 \times 10^{-7}$ \\
$2^{18}$ & $1.74 \times 10^{-7}$ & $3.49 \times 10^{-7}$ & $3.47 \times 10^{-7}$ \\
$2^{19}$ & $2.19 \times 10^{-7}$ & $3.52 \times 10^{-7}$ & $3.48 \times 10^{-7}$ \\
\hline
\end{tabular}

Table 5.1: Compression errors for the 2D problem using a truncation threshold of $10^{-7}$.

\begin{tabular}{|c|c|c|c|}
\hline Problem size & $\frac{\left\|A_{2}^{\mathcal{H}}-A_{1}^{\mathcal{H}}\right\|_{F}}{\left\|A_{1}^{\mathcal{H}}\right\|_{F}}$ & $\frac{\left\|A x-A_{1}^{\mathcal{H}} x\right\|}{\|A x\|}$ & $\frac{\left\|A x-A_{2}^{\mathcal{H}} x\right\|}{\|A x\|}$ \\
\hline \hline $2^{14}$ & $1.33 \times 10^{-3}$ & $9.10 \times 10^{-4}$ & $9.54 \times 10^{-4}$ \\
$2^{15}$ & $1.75 \times 10^{-3}$ & $9.49 \times 10^{-4}$ & $1.03 \times 10^{-3}$ \\
$2^{16}$ & $2.08 \times 10^{-3}$ & $9.19 \times 10^{-4}$ & $9.35 \times 10^{-4}$ \\
$2^{17}$ & $2.35 \times 10^{-3}$ & $9.62 \times 10^{-4}$ & $9.70 \times 10^{-4}$ \\
$2^{18}$ & $2.85 \times 10^{-3}$ & $9.78 \times 10^{-4}$ & $9.62 \times 10^{-4}$ \\
$2^{19}$ & $2.83 \times 10^{-3}$ & $9.76 \times 10^{-4}$ & $9.68 \times 10^{-4}$ \\
\hline
\end{tabular}

Table 5.2: Compression errors for the 3D problem using a truncation threshold of $10^{-3}$.

reduce their memory footprint in an accuracy controllable way.

Tables 5.1 and 5.2 show the compression errors for the 2D and 3D problems respectively. In this table, $A$ refers to the exact dense covariance (that is never formed but whose entries have analytical expressions from the underlying kernel), $A_{1}^{\mathcal{H}}$ refers to the hierarchal matrix approximation of the covariance generated using the generic Chebyshev polynomial basis described earlier, and $A_{2}^{\mathcal{H}}$ refers to the algebraically compressed covariance. The $A_{2}^{\mathcal{H}}$ matrices were generated using a truncation threshold of $10^{-7}$ for the $2 \mathrm{D}$ problems and $10^{-3}$ for the $3 \mathrm{D}$ problems. These thresholds were chosen to correspond to the errors that already existed in the $A_{1}^{\mathcal{H}}$ matrix approximation so that the algebraic compression does not introduce further approximation errors. Therefore the reduction in memory footprint comes purely from the generation of 
more efficient bases to represent the matrix.

The first column of Tables 5.1 and 5.2 shows the relative error between the original hierarchical matrix $A_{1}^{\mathcal{H}}$ and the compressed matrix $A_{2}^{\mathcal{H}}$ in the Frobenius norm. As expected, these errors are on the order of the truncation threshold used in the compression. I note here that the Frobenius norm error is computed, quite inexpensively, in the course of the truncation operation and does not require a separate post-processing operation. This is a useful feature for applications that require adaptive tolerances and fine error control inside chains of hierarchical operations. The second and third columns show the relative error pre- and post-compression respectively, measured in the 2-norm $\left\|A x-A^{\mathcal{H}} x\right\| /\|A x\|$ where $x$ is a random vector whose entries are uniformly distributed. For the large problems where it is too expensive to compute $A x$, I randomly sampled $10 \%$ of the rows and scaled the resulting error. As expected, comparing the second and third columns shows that compression with the appropriate thresholds had little to no effect on the accuracy of the resulting matrix.

I first profile and measure the performance of the orthogonalization kernel. Figure 5.4 shows the percentage of total operation time spent in each of the three phases of the orthogonalization in single and double precision. It is easy to see that the projection phase dominates the runtime for the larger problem sizes, making the computational kernel at the core of this operation, the batched matrix-matrix multiplication, the main performance limiter. On the P100 GPU and for small matrix sizes, the cuBLAS batched gemm routines show lackluster performance and stand to be improved; the single precision performance is actually quite close to that of the double precision. We see this effect in the runtimes of the orthogonalization in Figures $5.5 \mathrm{a}$ and $5.5 \mathrm{~b}$; however, the expected linear growth of the algorithm remains. As the kernels involved in this computation are compute-bound, Figures $5.5 \mathrm{c}$ and $5.5 \mathrm{~d}$ show the performance in single and double precision for the $2 \mathrm{D}$ and $3 \mathrm{D}$ problems in GFLOP/s. Overall however, the orthogonalization phase represents a relatively small 

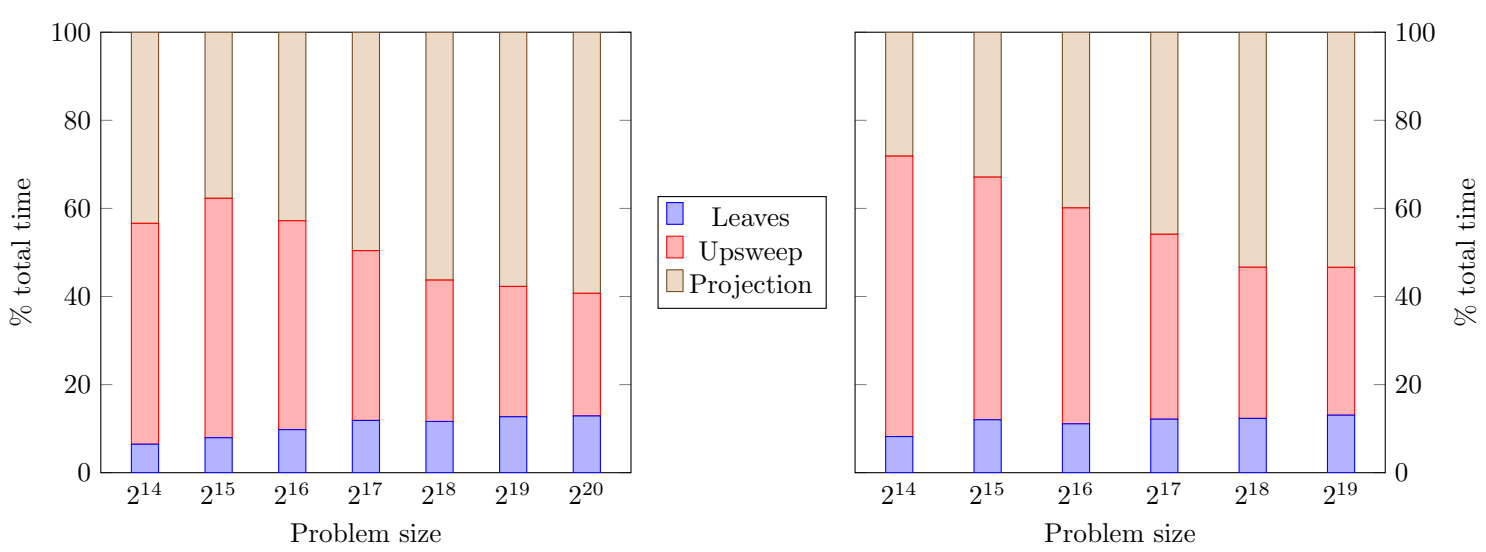

Figure 5.4: Breakdown of the orthogonalization phases in percentages of total operation time in single (left) and double (right) precision on a P100 GPU, showing that the projection phase dominates the runtime.

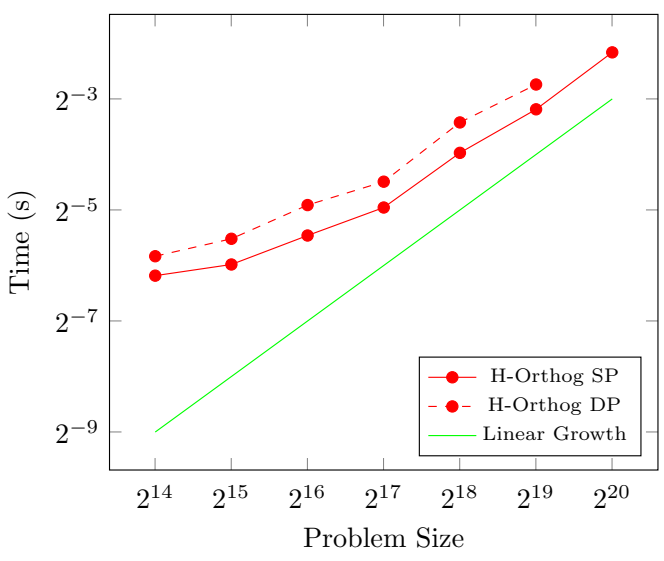

(a) Time for the orthogonalization in single and double precision for a 2D problem.

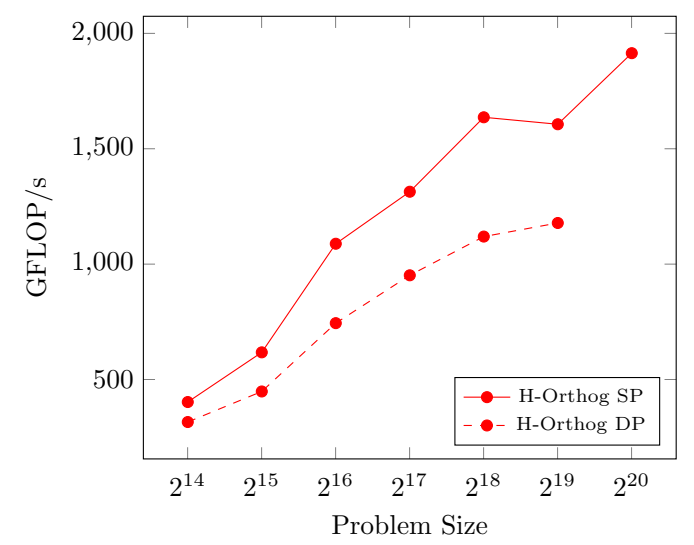

(c) Performance of orthogonalization in single and double precision for a $2 \mathrm{D}$ problem.

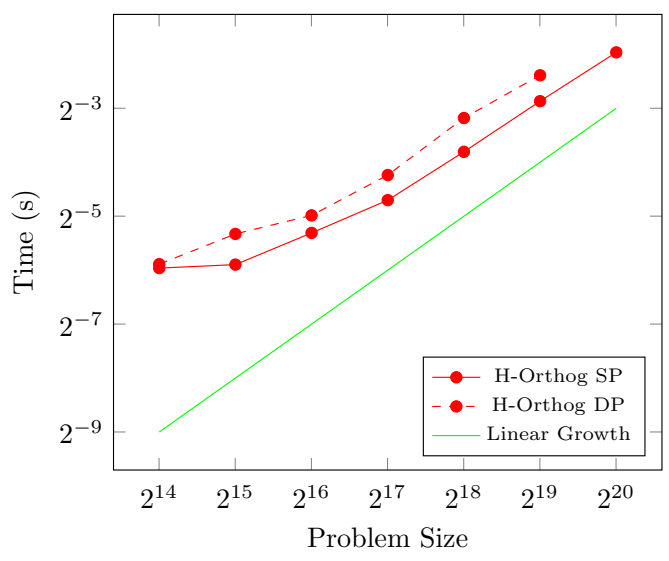

(b) Time for the orthogonalization in single and double precision for a 3D problem.

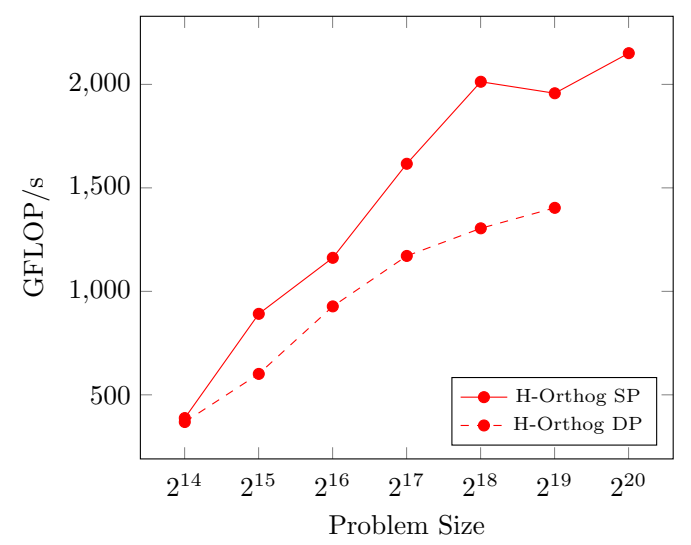

(d) Performance of orthogonalization in single and double precision for a 3D problem.

Figure 5.5: Runtime and achieved performance of the orthogonalization on a single P100 GPU. Note the asymptotic linear growth with problem size. Double and single precision times are closer to each other than expected due to the performance of the cuBLAS batched gemm routines. 

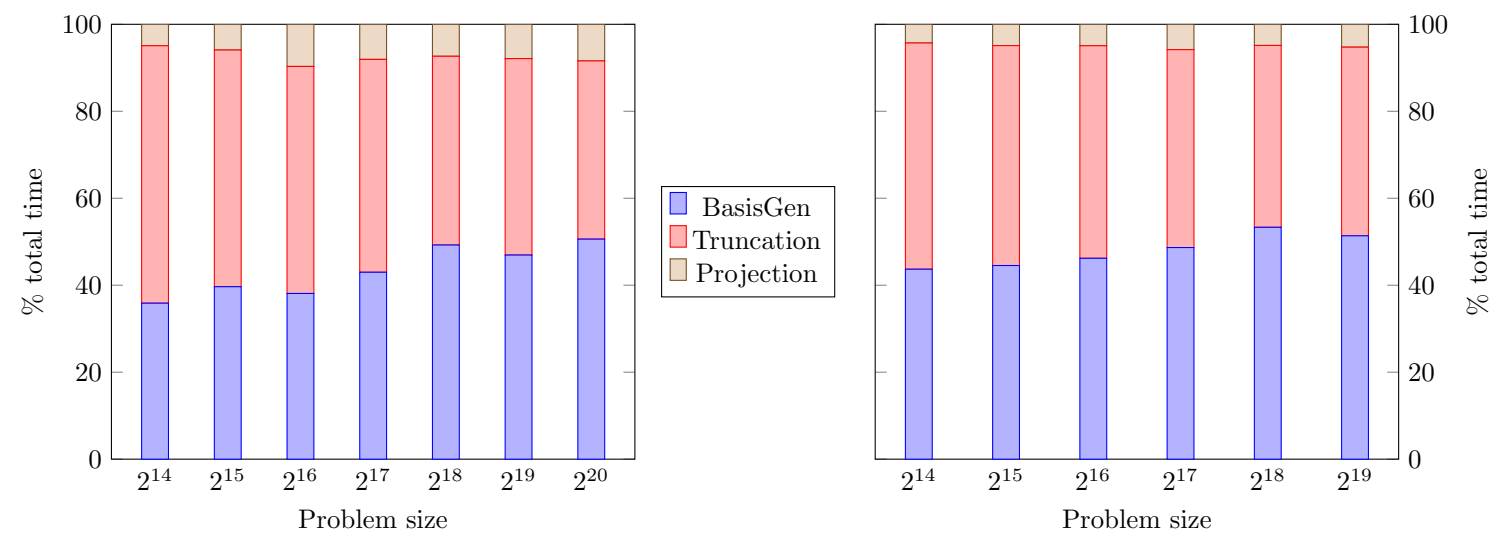

Figure 5.6: Breakdown of the compression phases in percentages of total operation time in single (left) and double (right) precision on a P100 GPU, showing that the truncation and basis generation phases dominate the computation.

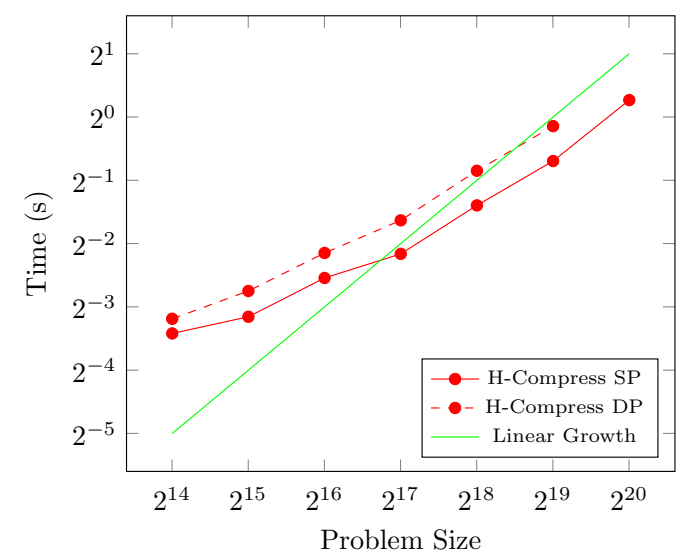

(a) Time for the compression in single and double precision for a 2D problem.

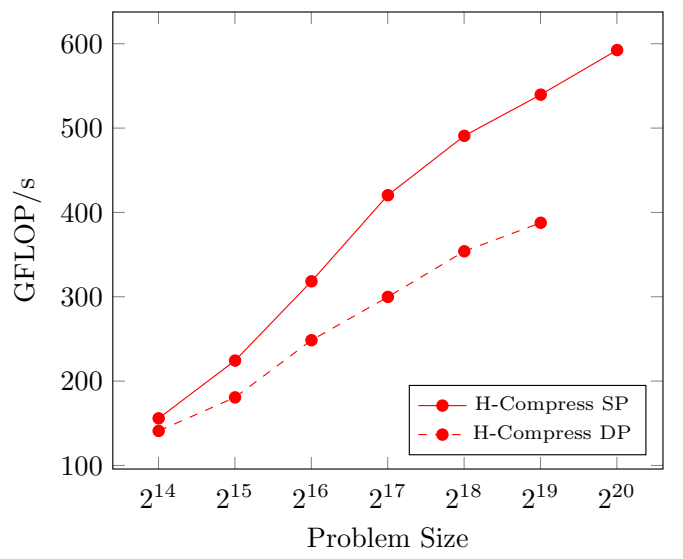

(c) Performance of compression in single and double precision for a $2 \mathrm{D}$ problem.

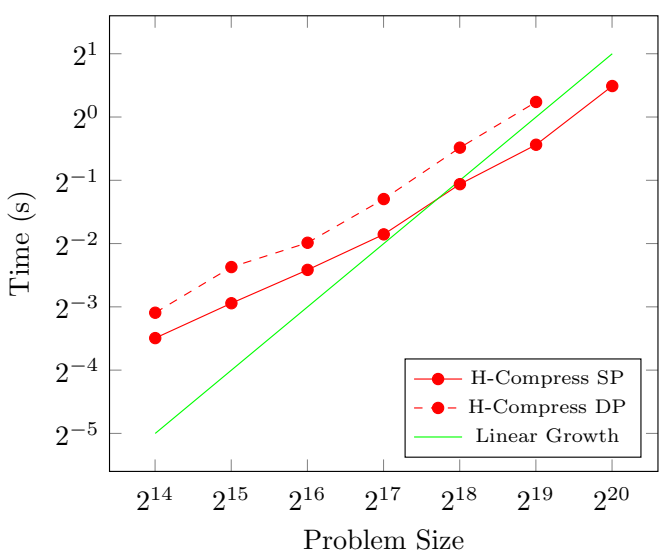

(b) Time for the compression in single and double precision for a 3D problem.

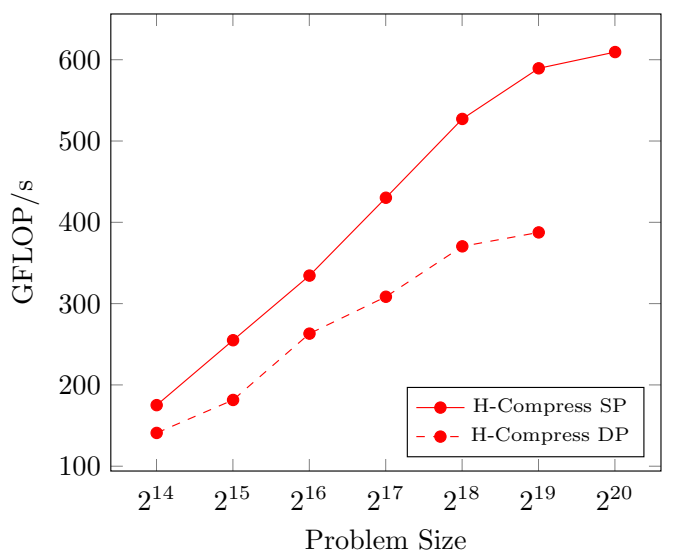

(d) Performance of compression in single and double precision for a 3D problem.

Figure 5.7: Runtime and achieved performance of compression on a single P100 GPU, showing asymptotically linear growth with problem size. 


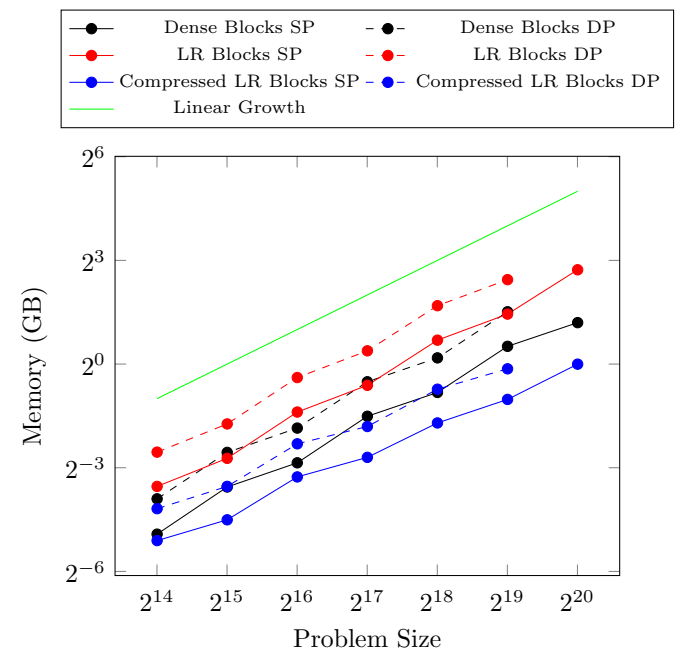

(a) Effect of the compression on memory for the $2 \mathrm{D}$ problem with a truncation threshold of $10^{-7}$.
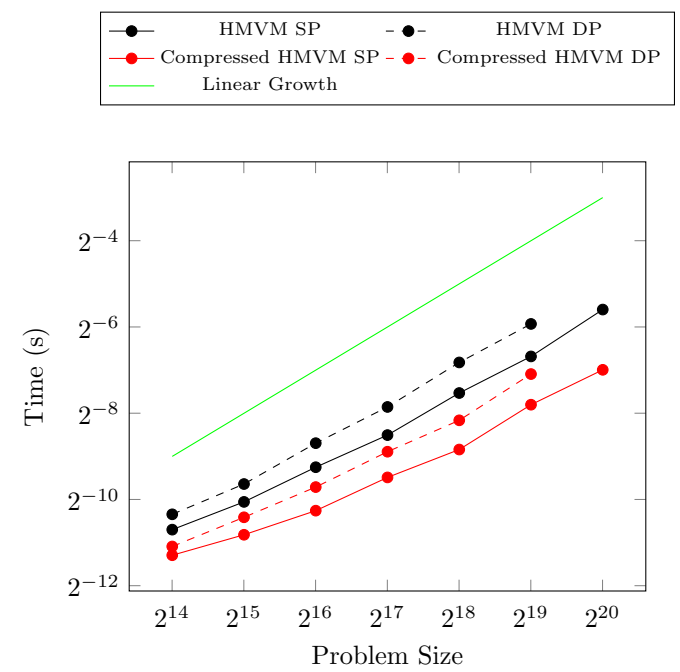

(c) Effect of the compression on hgemv for the $2 \mathrm{D}$ problem.
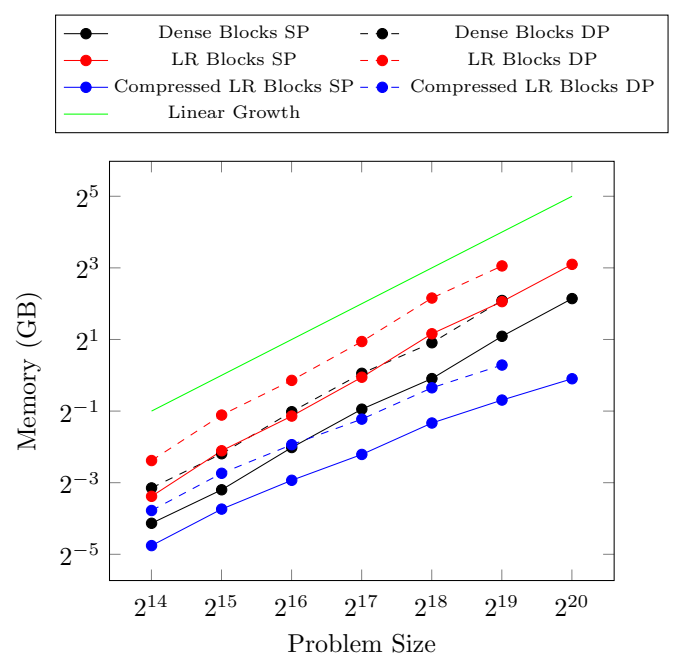

(b) Effect of the compression on memory for the $3 \mathrm{D}$ problem with a truncation threshold of $10^{-3}$.
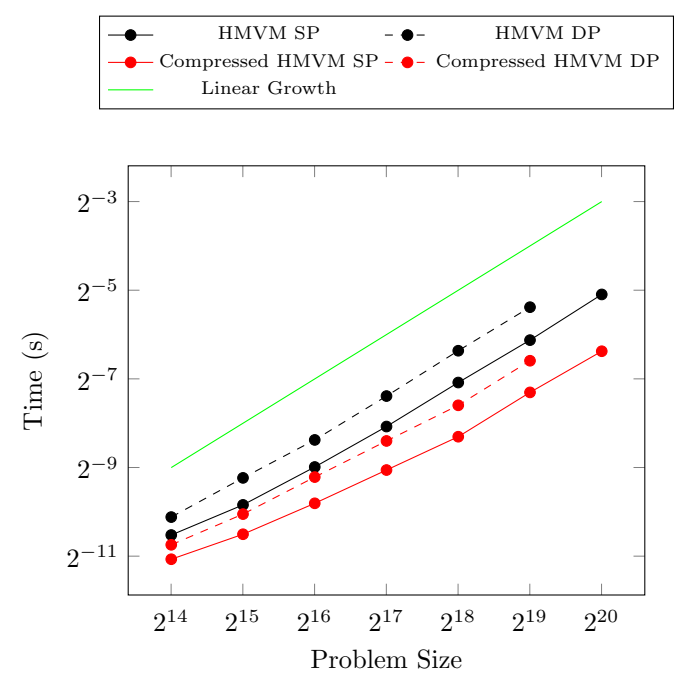

(d) Effect of the compression on hgemv for the $3 \mathrm{D}$ problem.

Figure 5.8: Effect of the compression on memory and runtime for the 2D and 3D problems, showing significant memory savings for the low rank portion and the hgemv time. 
percentage (10-15\%) of the total compression time.

Figure 5.6 shows the runtime profile of the compression in single and double precision where both truncation and basis generation phases are dominant due to the relatively costly SVD operations on the basis nodes and the QR decompositions on the coupling matrix data, especially when compared to the matrix-matrix multiplications of the projection phase. Figures $5.7 \mathrm{a}$ and $5.7 \mathrm{~b}$ show the asymptotic growth of the compression algorithm. Since this is also a compute-bound algorithm, I show the performance of the compression in GFLOP/s as the total number of operations executed by each batched kernel over total execution time in Figures $5.7 \mathrm{c}$ and $5.7 \mathrm{~d}$.

Figures $5.8 \mathrm{a}$ and $5.8 \mathrm{~b}$ show the significant memory savings achieved by the algebraic compression procedure. The compression also has a positive effect on the runtime of the hierarchical matrix arithmetic as shown in the improved runtimes of the matrix-vector operation in Figures $5.8 \mathrm{c}$ and $5.8 \mathrm{~d}$, 


\section{Chapter 6}

\section{Hierarchical Adaptive Randomized Approximation}

In some applications, such as the covariance matrices used in Chapters 4 and 5 . the hierarchical matrix approximation may be generated directly using analytically available kernels. But in many applications of interest, these analytical construction techniques are not applicable and algebraic methods are needed. The full dense form of the hierarchical matrix is far too expensive to generate and store, and therefore it is impractical to start from such a representation and then compress it. I therefore seek to adapt randomized methods to the generation of hierarchically low rank approximations of these large dense matrices. My primary interest is to develop the construction algorithm when the access to the matrix is available through matrixvector multiplication operations only. The methods I develop here directly construct the compressed hierarchical matrix form of the matrix without directly accessing its entries and build upon the previously developed hierarchical operations.

Two general strategies have been proposed for the construction of hierarchically low rank matrices from matrix-vector operations: a top-down strategy and a bottomup one. The top down approach [24, 25] starts from the coarsest level of the matrix

and "peels off" blocks from any given level by sampling with a random matrix that has a non-zero pattern specifically arranged to eliminate all but a particular block column. Once subspaces for these blocks are computed, a low rank approximation for them is generated and they are subtracted (peeled off) from the matrix. At the next level, the matrix is sampled with patterned random matrices and the contribution from the already factored coarser blocks is subtracted to provide the subspaces 
for block columns at that level. In [24] the pattern of sampling is obtained from the geometry of the grid that generated the hierarchical matrix, and the sampling pattern appropriate for a uniform Cartesian grid is presented. More general grids, or hierarchical matrices with general partitioning will require more careful patterned sampling and may decrease the amount of sampling that may be performed concurrently. In [25], an HSS representation $\left(\mathcal{H}^{2}\right.$ with a weak admissibility pattern) is used. This allows all the sampling for a given level to be done concurrently in parallel, but the resulting matrix may have large ranks because of the fixed weak partitioning.

The algorithm I propose below is in this same vein, but separates the sampling strategy from the target matrix being reconstructed. As I show below, I can sample using any convenient strategy and add low rank updates locally to the generally partitioned target matrix, while still keeping the nestedness of the basis. The separation of the structure sampling blocks from the structure of the matrix being constructed gives me flexibility to adapt the sampling strategy to the structure of the matrix and to the cost of matrix vector sampling.

The bottom-up approach [26] starts from the finest level of the hierarchy and constructs a basis for that level, and then moves up the hierarchy. In order to construct a level basis from the sampled matrix, arbitrary entries of the input matrix must be available to allow low rank interpolative decompositions of blocks to be generated efficiently. The need for having explicit values for entries may limit the applicability of this factorization scheme. Xia [27] used a similar strategy for compressing blocks in a nested dissection solver, and the STRUMPACK package [74, 75] also uses this randomized sampling strategy for compression of fronts into HSS form.

In this chapter, I discuss the details of a generalization of the ARA algorithm introduced in Chapter 3 that is used to construct a hierarchical representation of an $N \times N$ symmetric matrix using matrix vector products in a process I call hierarchical adaptive randomized approximation (HARA). The method leverages the ideas and 
routines developed for the ARA to produce low rank approximations of blocks of a hierarchical matrix in a top-down manner. It is worth noting that while bottomup algorithms [26] are asymptotically faster than top-down methods, differing by a factor of $O(\log N)$, they require the evaluation of the matrix entries in $O(1)$ time, a requirement that may not always be satisfied in applications.

The randomized approximation can be conceptually described as a two-phase process. In a first phase, a hierarchical matrix representation composed of low rank blocks is generated which is then, in a second phase, compressed into an $\mathcal{H}^{2}$ matrix with hierarchical bases. These two phases are not executed sequentially, however, but rather their applications are interleaved at every level. The low rank blocks generated in the first phase are accumulated level-by-level into the $\mathcal{H}^{2}$ matrix being constructed. There is never any need to store the complete hierarchical low rank matrix of the first phase, since it gets processed completely one level at a time. In order to perform the second phase accumulation by levels, the low rank blocks are turned into a set of local low rank updates that are then performed simultaneously on the whole matrix allowing high-performance batched kernels to be used in the accumulation and compression operations. If the ranks of the blocks generated in the first phase are too large to be processed in a single operation, the low rank updates can be split into smaller-sized updates and applied in sequence.

I call the matrix tree structure that is used to determine which blocks are approximated by low rank constructions in the first phase the sampling tree. The matrix tree structure of the hierarchical matrix that is being constructed by the HARA is called the approximation tree. Examples of these two trees for the structures show in Figures 6.2 and 6.3 can be seen in Figure 6.1 and it is worth noting that while these two trees need not share the same structure, the approximation tree must be a more refined version of the sampling tree.

Unlike previous approaches to peeling [24, 25], my proposed algorithm decouples 


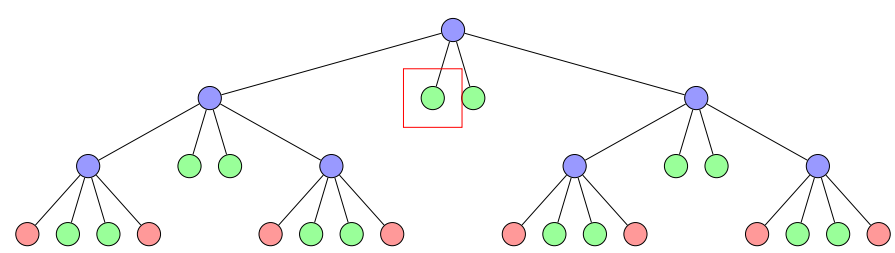

(a) Example of a sampling tree where a low rank representation of the highlighted node is first generated and then applied to the approximation tree below.

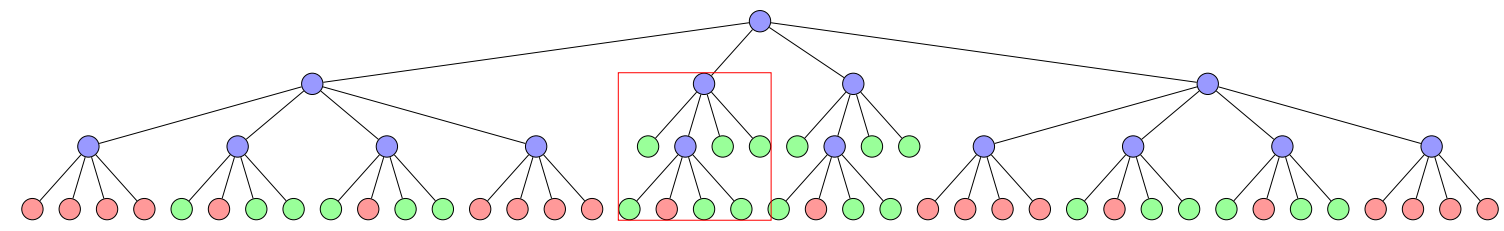

(b) Example of an approximation tree where a low rank update is applied to an inner node, resulting in updates to all the highlighted leaves of the subtree.

Figure 6.1: Examples of the sampling and approximation matrix trees and the application of a generated low rank update of the sampling tree to the approximation tree.

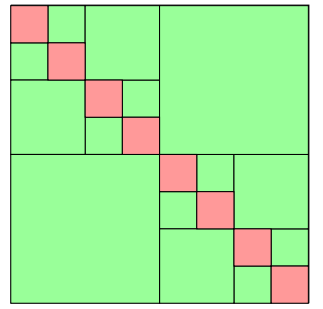

$A$

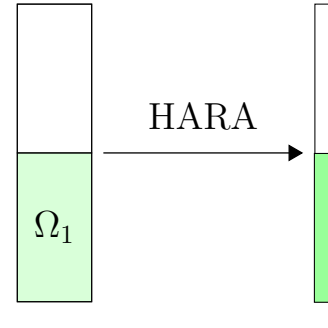

$\Omega$

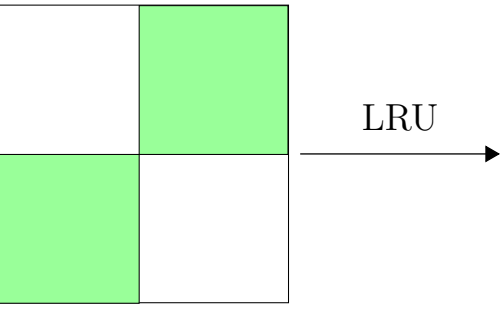

$Q B^{T}$

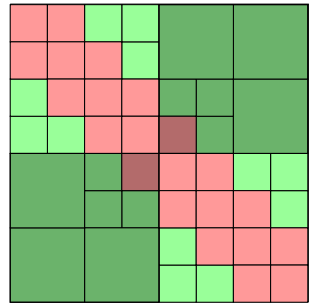

$A_{\mathcal{H}}^{1}$

Figure 6.2: The major phases of one level of the hierarchical adaptive randomized approximation. The left figure shows the matrix $A$ that is being approximated with the leaves of the sampling tree overlaid on it, as well as the structured random vectors generated by the sampling tree. These are used to generate the low rank updates of the center figure in a process similar to the ARA. Finally, the updates are applied to the hierarchical matrix $A_{\mathcal{H}}$ of the right figure, where the affected leaves of the matrix tree are highlighted in the shaded areas.

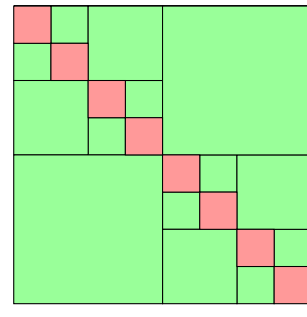

$A$

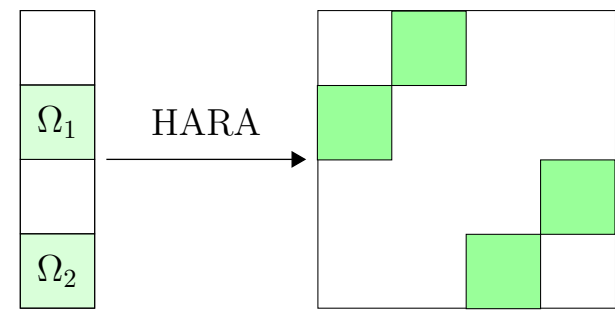

$\Omega$

$Q B^{T}$

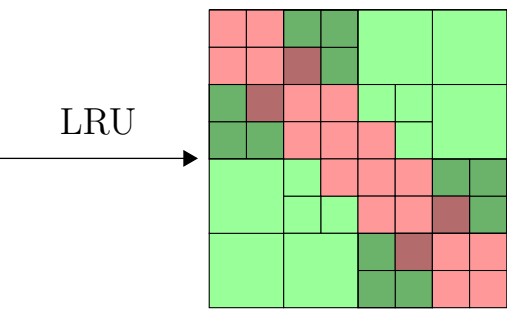

$A_{\mathcal{H}}^{2}$

Figure 6.3: Sampling the second level of the matrix and updating the approximation tree nodes. 
the sampling tree from the approximation tree. In [24], both the sampling and the target matrix use a standard admissibility structure, potentially requiring a large number of matrix-vector sampling operations, while in [25], both the sampling and the target use a weak admissibility structure, potentially resulting in a final matrix that has a large memory footprint because of large ranks in the off-diagonal blocks. The flexibility resulting from separating the sampling and approximation trees, allows me to adapt the sampling structure to the structure of the final matrix and to the cost of matrix-vector sampling. It is often more effective to sample in blocks whose sizes are larger than the block sizes that are best for the memory footprint of the final hierarchical matrix. It is also often convenient to use a simple sampling strategy while refining the approximation tree to fit the specific patterns needed by an application. This ability to choose separately the sampling and approximation trees is possible because the construction algorithm is formulated as a sequence of local low rank updates of the target matrix.

The blocks that form the leaves of the sampling tree are sampled one level at a time by computing the product of the entire matrix with randomly generated structured vectors based on the sampling tree. The samples then undergo a process similar to the ARA to produce a set of low rank updates that are added into the constructed hierarchical matrix, affecting all leaves of the approximation tree that lie within the sampled blocks. Finally, to reduce the memory footprint of the constructed hierarchical matrix after the low rank updates, the matrix is recompressed. This leads us to Algorithm 18 for the HARA using only matrix vector products on the input matrix. Figures 6.2 and 6.3 show the overall process for two levels of the HARA process. The computational complexity of the sampling phase is $O(k \log N)$ times the cost per single sample, where $k$ is an average rank of the sampled blocks at a given level. As I describe below, many of the sampling calls may be performed together on multiple samples and can result in substantial practical performance improvements: 
the number of multi-sample calls can be reduced to $O(\log N)$, i.e., a constant number of calls per level, each of which may be only a small factor more expensive that a single-sample call because of the higher arithmetic intensity involved. The accumulation and compression phase of the low rank updates of the construction operation has complexity $O\left(k^{2} N \log N\right)$, and may or may not dominate depending on problem context and required accuracy which directly impacts $k$.

In the following sections I will discuss the algorithms used to generate the low rank updates and their application to the constructed hierarchical matrix as well as their implementation on the GPU.

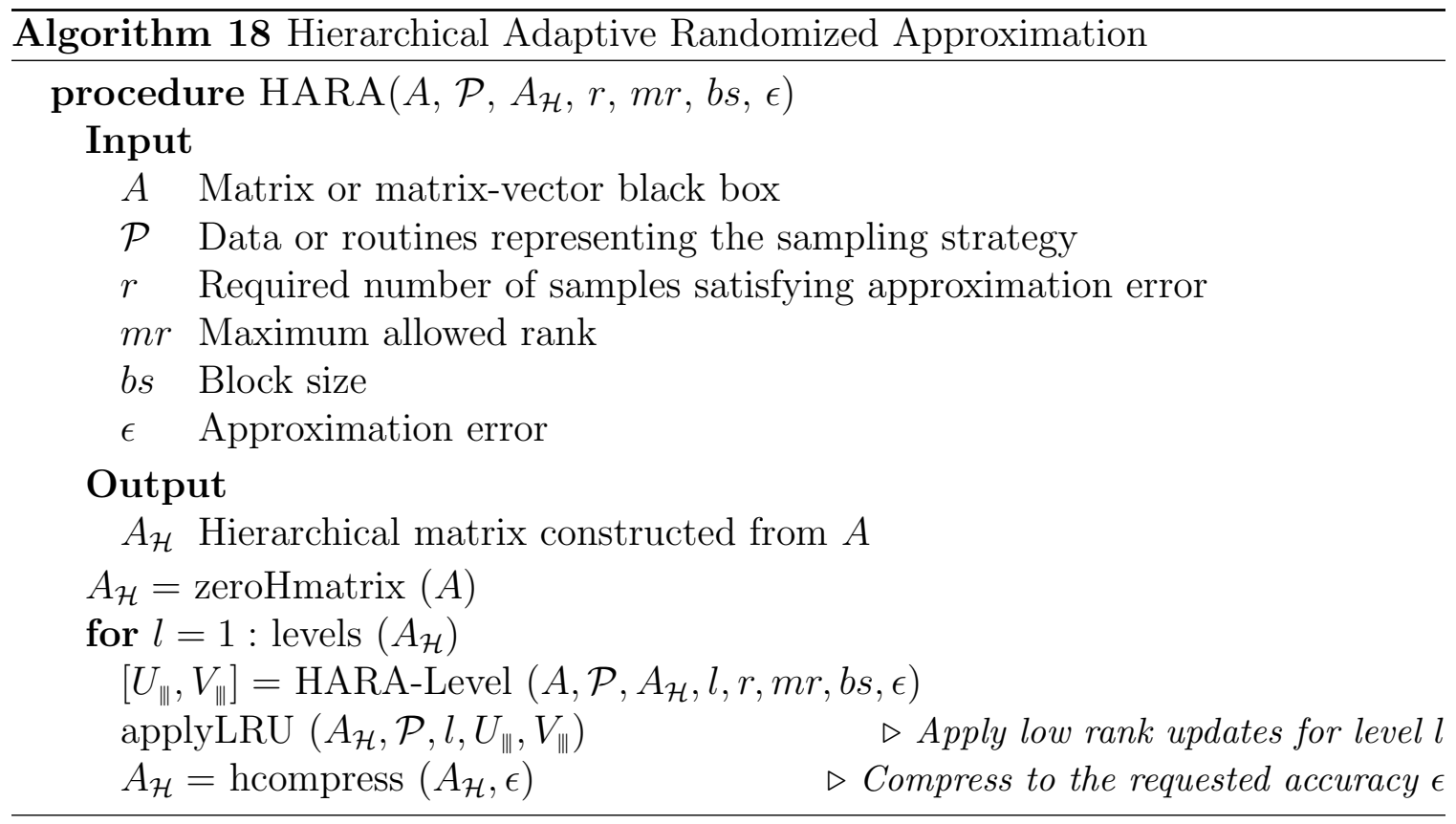

\subsection{Generating Low Rank Updates}

To approximate the blocks of the matrix that form the leaves of the sampling tree, I multiply the matrix by structured random vectors that allow me to sample those blocks without interference from other blocks. For example, if I need to sample the large upper right block of the simple sampling tree structure in Figure 6.2, I can generate a block of structured random vectors $\Omega$ where the top half that would be 
multiplied by the upper left block of the matrix is set to zero. The product of the matrix $A$ with $\Omega$ is then computed as:

$$
A \Omega=\left[\begin{array}{ll}
A_{1}^{1} & R_{1} \\
R_{1}^{T} & A_{2}^{1}
\end{array}\right]\left[\begin{array}{c}
0 \\
\Omega_{1}
\end{array}\right]=\left[\begin{array}{l}
R_{1} \Omega_{1} \\
A_{2}^{1} \Omega_{1}
\end{array}\right]
$$

where $R_{1}$ is the leaf I want to sample, and $A_{1}^{1}$ and $A_{2}^{1}$ are diagonal nodes. Ignoring the lower block $A_{2}^{1} \Omega_{1}$, I can see that the matrix vector product with the structured matrix $\Omega$ yields the product $R_{1} \Omega_{1}$ of the block I need to sample with a set of random vectors, the key ingredient for ARA to produce the low rank approximation of the block. Although this process can be repeated for every leaf in the sampling tree, it will obviously not be efficient to do so, since sampling each block will involve a matrix vector multiplication of the entire matrix $A$. Instead, I would like to be able to sample as many blocks as possible for each matrix vector product that I perform.

\subsubsection{Sampling Strategy}

It is obvious that I can simultaneously sample all blocks within a block column $s$ at level $l$ by only filling in the entries of the random input vector corresponding to the index set of $s$; however, limiting myself to one block column per sample would lead to $O(n)$ growth in the number of needed matrix vector products. When attempting to sample two blocks belonging to different block columns $R_{t_{1} s_{1}}^{l}$ and $R_{t_{2} s_{2}}^{l}$ at level $l$ of the sampling tree using a single matrix vector product, I must ensure that other blocks from the matrix do not interfere with the sampling; i.e., that there is no contribution to the output vector from matrix blocks in the intervals defined by $\left(t_{1}, s_{2}\right)$ and $\left(t_{2}, s_{1}\right)$. To simultaneously sample multiple blocks of a matrix, any interference must be dealt with using a combination of two sampling techniques.

The first technique sets the entries of the random input vector that will interact with the unwanted blocks to zero as I have done in the previous section. The second 
technique sets the interfering blocks themselves to zero by subtracting or "peeling" them out from the input matrix. Since I lack the exact representation of the blocks, I use the generated hierarchical matrix approximation instead. While this does introduce some errors into the approximation which could potentially accumulate as I go down the tree, I have observed in my experiments that the approximation error of the resulting hierarchical matrix satisfies the chosen error threshold. This could be a result of using a uniform rank for each level of the matrix, set to be the largest rank of all blocks at that level (due to the lack of necessary non-uniform batched GPU routines). The study and analysis of these errors and their effect on the choices for error thresholds at each level will be the focus of future work.

I peel the blocks of the matrix defined by the sampling tree in a top-down manner, generating approximations of all nodes one level at a time. This allows me to limit the search for interfering blocks to nodes that belong to the same level, since the nodes at higher level are assumed to have already been peeled off. This leads us to the following general strategy: for each level $l$ in the sampling tree, determine groups of blocks that can be sampled simultaneously assuming that the matrix blocks in the upper levels have been peeled off and generate structured random input vectors based on the index set of each group of blocks. The assumption that the higher levels have been peeled off is made valid by sampling the matrix $A^{l}=A-A_{\mathcal{H}}^{l}$, where $A_{\mathcal{H}}^{l}$ contains all the previously approximated levels. If I assume the simple weak admissibility structure of the symmetric matrix in Figure 6.2, I can simultaneously sample all the off-diagonal blocks at each level of the sampling tree with the same matrix vector product. This can be seen in Figure 6.3, where all the off-diagonal blocks are sampled simultaneously. The dense diagonal blocks are extracted directly by multiplying $A^{l}$ by a block column matrix where each block is the identity matrix.

More general sampling tree structures, i.e., peeling strategies, would need to efficiently determine which block column nodes can be grouped together based on 
whether all their respective nodes do not interfere with each other. A key observation here is that at every level the coupling matrix may be viewed as block sparse, where blocks corresponding to coarser levels have been eliminated. The problem of reducing the number of separate sampling calls can then be formulated as a graph coloring problem in the spirit of the CPR algorithm [76] for partitioning matrix columns in the computation of sparse Jacobians. The generation of a small number of groups of structurally orthogonal columns may be done using a greedy strategy as was done in [76], or by using more sophisticated heuristics in the solution of the graph coloring problem. Coleman and More [77], for example, write the problem as a distance-1 graph coloring problem. In this formulation, a column intersection graph of a matrix is used where a column in the matrix corresponds to a vertex, and an edge exists between two vertices if their corresponding columns have non-zeros in a common row. In the matrix peeling problem, structurally non-orthogonal column blocks would correspond to having blocks not yet peeled in a common block row, and this is done at every level of the matrix hierarchy. For symmetric matrices, path coloring [78] may be used to generate more effective groups of structurally orthogonal columns. A detailed survey and discussion of this general problem appears in [79]. This will be the focus of future work.

\subsubsection{GPU Implementation}

Algorithm 19 details the generation of the low rank updates for a level of the matrix. It is structurally very similar to the ARA algorithm, needing only a few changes made to the sampling process and low rank approximation management. I first define my sampling strategy $\mathcal{P}$ as a set of data or routines that determine which blocks can be sampled simultaneously based on the structure of the sampling tree. In the simple

case of a sampling tree having a weak admissibility structure, the sampling strategy $\mathcal{P}$ would simply return all the off-diagonal blocks in the level $l$ in a single batch. This 
is due to the fact that for the simple structure generated by weak admissibility, all offdiagonal blocks within a level can be sampled at the same time without interference. As mentioned in Section 6.1.1, more complex sampling strategies for standard admissibility structures would potentially produce multiple batches of blocks that can be sampled simultaneously, but the basic idea remains the same, where only the contents and size of the batched blocks would change. Pointer and dimension data for the low rank updates $U$ and $V$ are marshaled for the current level $l$ based on $\mathcal{P}$. A simplified version of this marshaling routine is shown in Algorithm 20 where pointer data is generated from the indexes that define the blocks generated by $\mathcal{P}$. In a similar manner, the random input vector is generated by first marshaling the necessary pointer and dimension data from $\mathcal{P}$ and then passing the data to a batched block random number generator (implemented using device level CURAND routines). Sampling is handled by a black box routine that performs the matrix vector multiplication of the matrix $A^{l}=A-A_{\mathcal{H}}^{l}$ with the generated random vectors. This can obviously be split into the original black box routine and a regular hierarchical matrix vector product. Finally, the right factor $V$ is produced by first marshaling the necessary data from the left factor $U$ using $\mathcal{P}$, clearing the data using a batched routine and multiplying $A^{l}$ by $U$.

\subsection{Applying Low Rank Updates}

After generating the low rank approximations of the leaves of the sampling tree, they must be added to the constructed hierarchical matrix as low rank updates while maintaining the nested basis property of the basis tree. As mentioned in the previous section, the sampling and approximation trees do not need to have the same structure, making it possible to add low rank updates to inner nodes of the approximation tree. These updates can be performed in linear complexity [80] even for matrices with general partitioned structure, as long as the number of blocks per row is bounded 
at every level in the hierarchy, a condition that is naturally satisfied by matrices that admit hierarchical representations. In this section, I discuss the details of the algorithm used to apply low rank updates to the nodes of the approximation tree as well as its GPU implementation.

\subsubsection{Updating the Basis Nodes}

Given a low rank update $R_{i}=Q_{i} B_{i}^{T}$ to a node $A_{t s}^{l}$ of the approximation tree, I must update the corresponding nodes $U_{t}^{l}$ and $V_{s}^{l}$ in the basis tree while maintaining the nested basis property. Assuming the matrix is symmetric and that for every low rank update $R_{i}=Q_{i} B_{i}^{T}$ there must be a corresponding low rank update $R_{i}^{T}=B_{i} Q_{i}^{T}$ to maintain the symmetry of the matrix, I will focus on the changes to the $U$ basis tree. The non-symmetric case follows the same algorithm with updates to the $V$ basis tree.

If $U_{t}$ is a leaf node, the update to the basis is trivial; I simply append the columns of $Q_{i}$ to the columns of the explicitly stored basis node:

$$
\bar{U}_{t}^{l}=\left[\begin{array}{ll}
U_{t}^{l} & Q_{i}
\end{array}\right],
$$

where $\bar{U}_{t}^{l}$ is the new basis node. If $U_{t}$ is an inner node which is only stored implicitly by the transfer matrices of its children, the update $Q_{i}$ is split into block rows and applied recursively to its children. If node $t$ has two children $c_{1}$ and $c_{2}$, I can represent the new node as:

$$
\bar{U}_{t}^{l}=\left[\begin{array}{ll}
U_{t}^{l} & Q_{i}
\end{array}\right]=\left[\begin{array}{ll}
U_{c_{1}}^{l+1} E_{c_{1}}^{l+1} & Q_{i_{1}} \\
U_{c_{2}}^{l+1} E_{c_{2}}^{l+1} & Q_{i_{2}}
\end{array}\right]=\left[\begin{array}{cccc}
U_{c_{1}}^{l+1} & Q_{i_{1}} & 0 & 0 \\
0 & 0 & U_{c_{2}}^{l+1} & Q_{i_{2}}
\end{array}\right]\left[\begin{array}{cc}
E_{c_{1}}^{l+1} & 0 \\
0 & I \\
E_{c_{2}}^{l+1} & 0 \\
0 & I
\end{array}\right],
$$

where $Q_{i_{1}}$ and $Q_{i_{2}}$ are the block rows of $Q_{i}$ corresponding to the index sets of the 
children nodes $c_{1}$ and $c_{2}$ respectively. The above equation shows us that passing on the block rows to the children and replacing the transfer matrices of the node by block diagonal matrices, where the upper block is the old transfer matrix and the lower block is the identity matrix, allows me to implicitly append the columns of the update to the columns of the basis node. Finally, to maintain the nested basis property, the rows of the transfer matrices of the parent node which was not affected by the update are padded with zeros. For example, the parent basis node $t^{+}$for an updated node $t_{1}$ whose sibling is $t_{2}$ is represented as:

$$
U_{t^{+}}^{l-1}=\left[\begin{array}{cc}
U_{t_{1}}^{l} & 0 \\
0 & U_{t_{2}}^{l}
\end{array}\right]\left[\begin{array}{l}
E_{t_{1}}^{l+1} \\
E_{t_{2}}^{l+1}
\end{array}\right]=\left[\begin{array}{ccc}
U_{t_{1}}^{l} & Q_{i} & 0 \\
0 & 0 & U_{t_{2}}^{l}
\end{array}\right]\left[\begin{array}{c}
E_{t_{1}}^{l+1} \\
0 \\
E_{t_{2}}^{l+1}
\end{array}\right]
$$

\subsubsection{Updating the Coupling Nodes}

After the basis is properly updated, I must update the coupling matrices of the hierarchical matrix. If the updated node $A_{t s}^{l}$ is a leaf in the approximation tree, the update simply involves replacing the coupling matrix of the node with a block diagonal matrix where the upper block is the original coupling matrix and the lower block is the identity:

$$
A_{t s}^{l}+Q_{i} B_{i}^{T}=U_{t}^{l} S_{t s}^{l} V_{s}^{l}+Q_{i} B_{i}^{T}=\left[\begin{array}{ll}
U_{s}^{l} & Q_{i}
\end{array}\right]\left[\begin{array}{cc}
S_{t s}^{l} & 0 \\
0 & I
\end{array}\right]\left[\begin{array}{ll}
V_{t}^{l} & B_{i}
\end{array}\right]=\bar{U}_{t}^{l} \bar{S}_{t s}^{l} \bar{V}_{s}^{l}
$$

where $\bar{S}_{t s}$ is the new coupling node. If the updated node is an inner node, then the aforementioned update is carried out on all of its children. If a child node happens to be a dense block, then the update is carried out explicitly using matrix matrix multiplication. 


\subsubsection{GPU Implementation}

Algorithm 21 describes the major steps of the GPU implementation of applying the low rank update to a level of the hierarchical matrix. To efficiently implement the application of low rank updates on the GPU, I again split the operation into a marshaling phase where pointer and dimension data is generated and an execution phase using high performance batched GPU routines. The low rank updates are generated only as matrix data in the form $R_{i}=Q_{i} B_{i}^{T}$ and the index $i$ of the node in the sampling tree corresponding to the approximated block. Since the sampling and approximation trees can be different, the first step determines the node indexes of the approximation tree corresponding to each sampling tree node index in the update list. This can be done very efficiently using the lower_bound Thrust routine with the Morton order index of each node within the current level used as the search key to search for all nodes indexes simultaneously with a single kernel call. Using the approximation tree node index data of the updates, I flag the affected nodes in the basis tree and then use a single transformation Thrust kernel per level of the tree to flag the children of the nodes. Flagged inner nodes are updated by first copying the original transfer matrices using a batch block copy kernel followed by a batched kernel that sets the lower block diagonal to the identity matrix as discussed in Section 6.2.1. The flagged leaf nodes are updated using two batched copy kernels for the original leaf data and the appropriate block rows of the low rank update. The coupling matrices are updated similarly, where updated nodes are flagged in one pass and the children are flagged one level at a time. Flagged dense blocks are updated using batch matrix matrix multiplication kernels from cuBLAS.

\subsection{Compression to Reduce Rank}

To maintain the optimal complexity of the hierarchical operations and reduce the memory consumption of the hierarchical matrix after ranks increase due to the low 
rank updates, the matrix must be compressed. Compression involves computing a new compact basis for the blocks of the matrix followed by a projection of the blocks into the new basis. The details of the algorithm are described in 5 . I briefly summarize the major phases of the compression here in order to highlight an optimization to the algorithm that can be employed when low rank updates are applied top-down one level at a time, as I do in my construction procedure.

The algorithm first orthogonalizes the bases. A basis is orthogonalized by performing a QR decomposition at the leaves of the basis and sweeping up the tree to orthogonalize the inner nodes using the nested basis property:

$$
U_{t^{+}}^{l-1}=\left[\begin{array}{cc}
U_{t_{1}}^{l} & \\
& U_{t_{2}}^{l}
\end{array}\right]\left[\begin{array}{c}
E_{t_{1}}^{l} \\
E_{t_{2}}^{l}
\end{array}\right]=\left[\begin{array}{cc}
\dot{U}_{t_{1}}^{l} & \\
& \dot{U}_{t_{2}}^{l}
\end{array}\right]\left[\begin{array}{c}
T_{t_{1}}^{l} E_{t_{1}}^{l} \\
T_{t_{2}}^{l} E_{t_{2}}^{l}
\end{array}\right]=\left[\begin{array}{cc}
\dot{U}_{t_{1}}^{l} & \\
& \dot{U}_{t_{2}}^{l}
\end{array}\right] Z_{t^{+}},
$$

where $\dot{U}$ is the new orthogonal basis and the $T$ matrices are the triangular factors of the QR decompositions of the basis nodes. A QR decomposition is then performed on each generated $Z$ matrix to produce the $T$ matrices of the current level. The appropriate block rows of the orthogonal factor replace the transfer matrices of the node, finalizing the orthogonalization of the node. The $T$ matrices are then used in a projection phase to update the coupling matrices:

$$
U_{t}^{l} S_{t s}^{l} V_{s}^{l T}=\dot{U}_{t}^{l}\left(T_{t}^{l} S_{t s}^{l} T_{t}^{l T}\right) \dot{V}_{s}^{l T}=\dot{U}_{t}^{l} \dot{S}_{t s}^{l} \dot{V}_{s}^{l T}
$$

Once the basis is orthogonalized, I generate an optimal basis from the data of the matrix in a downsweep pass and compress the basis in an operation that is structurally very similar to the orthogonalization, using the SVD of the nodes instead of the QR decomposition and truncating the singular values that are smaller than my truncation threshold $\epsilon$. These operations typically sweep up and down the entire tree. However, in the context of compression after low rank updates, one can save a 
significant amount of computation by taking advantage of the fact that I update in a top-down manner, where the levels above the updated level remain untouched. Consider an orthogonal basis tree that has been updated at level $l$. To re-orthogonalize the basis, I proceed with the regular algorithm until I reach level $l$. I know that the nodes above that level remain untouched by the update and therefore are still orthogonal. For an orthogonal node $U_{t^{+}}^{l-1}$, the matrix $Z_{t^{+}}$from Equation 6.6 must be orthogonal, since the block diagonal matrix $\left[\begin{array}{cc}\dot{U}_{t_{1}}^{l} & \\ & \\ & \dot{U}_{t_{2}}^{l}\end{array}\right]$ is also orthogonal. Orthogonalizing the matrix $Z_{t^{+}}$in this case would simply produce the identity matrix as its triangular factor $T$, resulting in no changes to the basis nodes at higher levels or their associated coupling matrices. Therefore, the process can stop at this level by directly replacing the transfer matrices $E_{t_{1}}^{l}$ and $E_{t_{2}}^{l}$ with the block rows $T_{t_{1}}^{l} E_{t_{1}}^{l}$ and $T_{t_{2}}^{l} E_{t_{2}}^{l}$ respectively. Similarly, the compression process can be sped up by starting the optimal basis downsweep at the updated level, since the matrix data of the upper levels did not change, and the upsweep can be cut short with similar reasoning to the orthogonalization.

\subsection{Numerical Experiments}

To analyze the performance of the HARA algorithm, I present results from three tests. The first one is a self-reconstruction experiment aiming to reconstruct approximations of a given hierarchical matrix with different accuracy thresholds by accessing it only through matrix-vector products. The second test is a matrix-matrix multiplication operation, where I aim to construct the product by sampling it via two matrixvector operations involving the matrix operands. The third test computes the Schur complement of a discretization of a Poisson operator. All experiments were run on a 16 GB Pascal P100 GPU and performance results were averaged over 10 runs.

Even though matrix-vector operations involving single vectors are fast, both in 
the $O(N)$ asymptotic sense and in practical running times on GPUs [81, they are bandwidth-limited computations and therefore cannot optimally exploit GPU capabilities. I have implemented a kernel that allows multiple matrix-vector products to be performed simultaneously to allow for increased arithmetic intensity and avoid bandwidth-limited bounds on performance. I use this kernel in the first two experiments.

\subsubsection{Construction of a Spatial Statistics Covariance Matrix}

For the first experiment, I generate covariance matrices for a 2D spatial Gaussian process with a spatially varying density of point distribution and an isotropic exponential kernel with correlation length 0.1 . Hierarchical representations of the formally dense $N \times N$ covariance matrices are formed analytically by first clustering the points in a KD-tree using a mean split giving me the hierarchical index sets of the basis trees. The basis vectors and transfer nodes are generated using Chebyshev interpolation [82]. The approximation tree is constructed using a dual traversal of the basis tree [69], and the coupling matrices are generated by evaluating the kernel at the interpolation points. The matrix is constructed to a relative accuracy of $10^{-7}$ in the Frobenius norm.

In this experiment, the black box sampling matrix vector multiplication routine is simply the product of the hierarchical covariance matrix with the input vector. The constructed hierarchical matrix has a leaf size of 64 and a refined matrix tree shown in Figure 6.4b, The sampling tree used was the simple weak admissibility sampling structure shown in Figure 6.4a. I used a block size $b s$ of 32 and two approximation thresholds for HARA at $10^{-2}$ and $10^{-4}$ to study the effect of the error threshold on the runtime as well as on the computed ranks and samples. To verify that the constructed hierarchical matrix $A_{\mathcal{H}}$ achieves the requested accuracy, I approximate the difference between $A_{\mathcal{H}}$ and the black box $A$ by taking the maximum relative error 


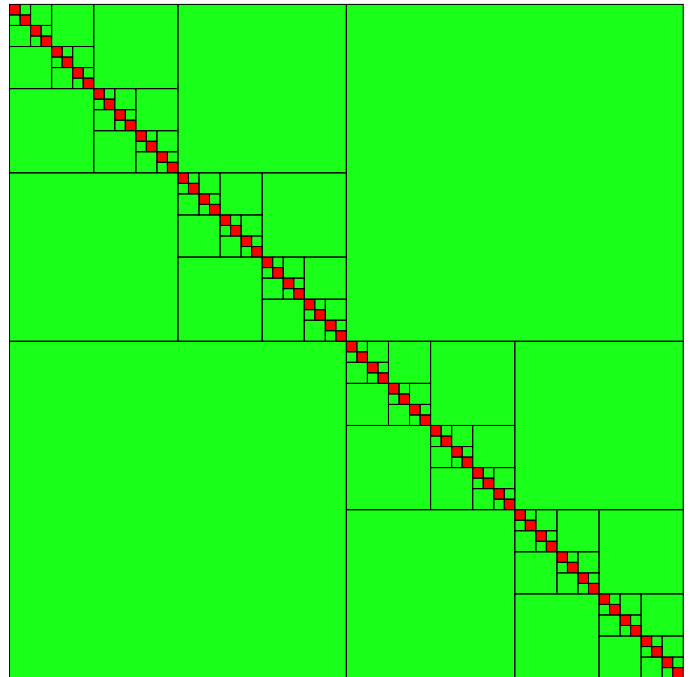

(a) The leaves of the weak admissibility sampling tree for a problem size of $2^{12}$.

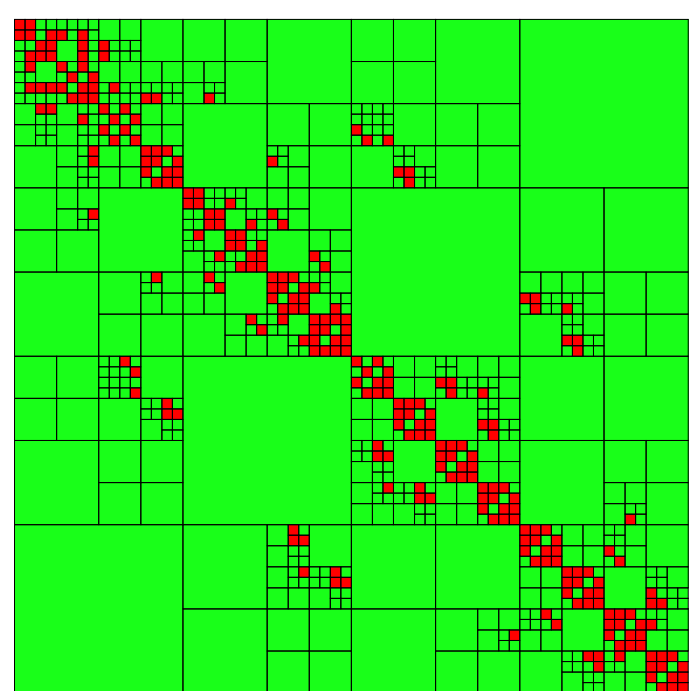

(b) The leaves of the matrix approximation tree for a problem size of $2^{12}$.

Figure 6.4: Sampling and approximation trees used to construct a hierarchical matrix.

of the product of the difference with a set of 10 normalized random vectors $x_{i}$

$$
\bar{\epsilon}=\max _{i=1 \ldots 10} \frac{\left\|\left(A_{\mathcal{H}}-A\right) x_{i}\right\|}{\left\|A x_{i}\right\|}
$$

Figure 6.5a shows the sum of all samples taken for each level of the hierarchical matrix. The tighter error threshold clearly requires significantly more samples to approximate the matrix and exhibits faster growth in the number of samples as the problem size increases. This is reflected in the overall runtimes of the algorithm for each threshold as seen in Figure 6.5b where the approximation with the higher accuracy take almost $7 \mathrm{x}$ the time to complete when compared to the lower accuracy approximation. For comparison, my single-threaded reference implementation of the compression on a $2.4 \mathrm{GHz}$ Broadwell CPU for a problem size of $2^{14}$ is completed in about 12 seconds for an accuracy of $10^{-2}$ and 370 seconds for an accuracy of $10^{-4}$. Figure 6.6 breaks down the computation into its four major phases. I note that most of the time is spent in the sampling phase for the lower accuracy approximation but in the compression phase for the higher accuracy. This difference is primarily due to the 


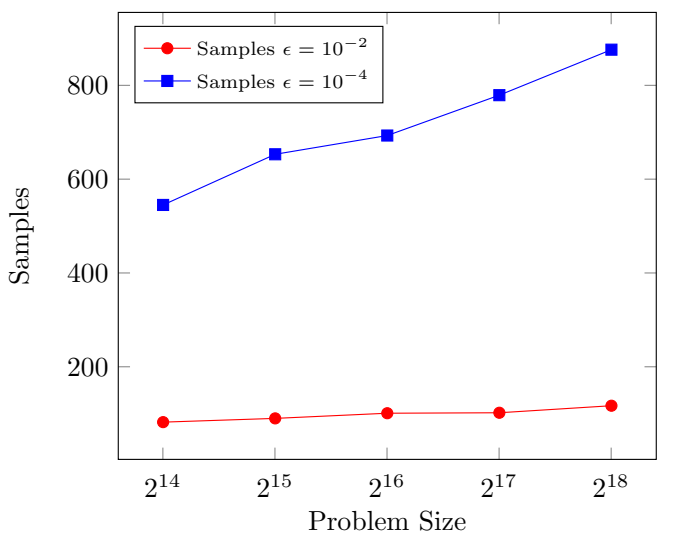

(a) Number of samples required to construct an $\mathcal{H}^{2}$ matrix representation to a specified accuracy.

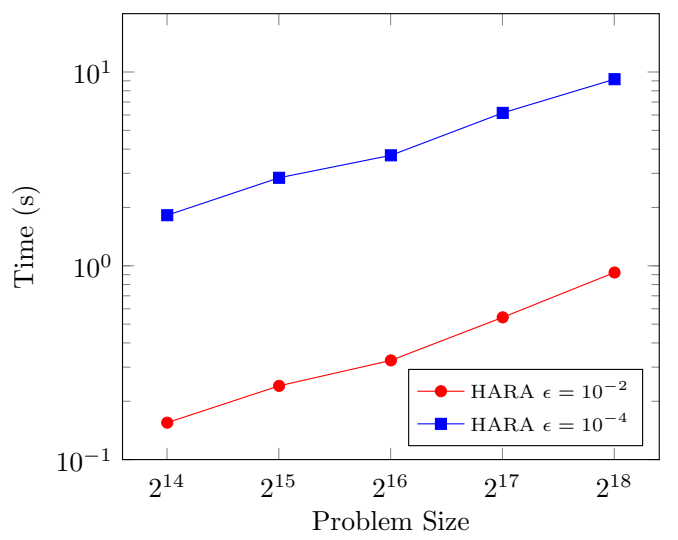

(b) GPU time for the construction.

Figure 6.5: Scalability and performance of HARA on a representative covariance matrix from spatial statistics.

significantly larger ranks in the constructed hierarchical matrix as shown in Figure 6.7, where the runtimes of the relatively inefficient SVDs used in the compression dominate those of the extremely efficient matrix multiplications of the sampling.

Figure 6.8 shows the achieved performance of the overall construction operation. It is possible to further optimize the GPU kernels inside the compression operations by using a randomized SVD following batched ARA approximations, but this requires some additional non-uniform batched routines in my software infrastructure, and I leave this as future work.

\subsubsection{Hierarchical Matrix-matrix Multiplication}

In this second experiment, I show that the HARA construction routine can perform a matrix-matrix multiplication operation by constructing the square of a hierarchical matrix. I use a covariance matrix similar to the one in the previous section with a randomly perturbed point set on a regular $[0,1]^{2}$ grid.

This experiment illustrates the ability of the method to compute a general matrix expression, given only the ability to multiply the expression by a vector. The matrix 

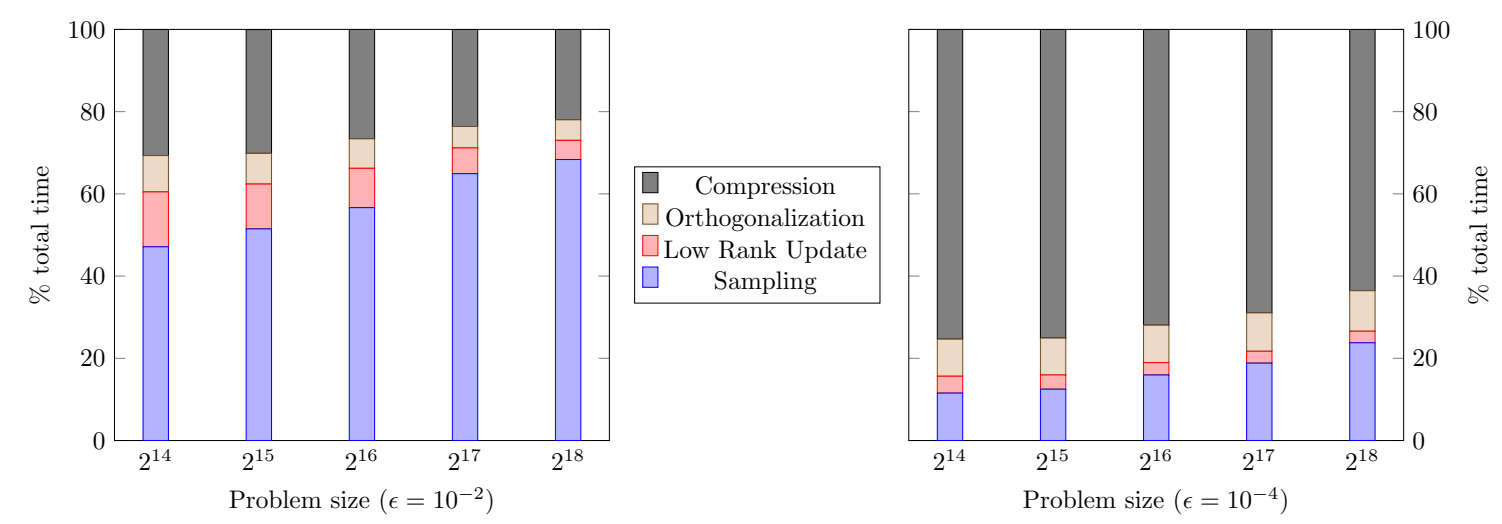

Figure 6.6: Profile showing relative costs of the different phases of the reconstruction operation.

squaring operation also allows me to examine the effect of the faster decay of singular values that accompanies the squaring on the performance of the algorithm. In randomized matrix-matrix multiplication operation, the number of samples needed depends on the ranks of the blocks in the resulting product and not on the ranks of the operands.

The black box sampling routine in this case simply performs two hierarchical matrix vector multiplications using an intermediate vector $z$ to store the first product:

$$
y=A^{2} x=A(A x)=A z
$$

I use the HARA algorithm and the same hierarchical matrix parameters for $A$ as in the first experiment. As expected, the number of required samples for both $10^{-2}$ and $10^{-4}$ error thresholds decreases in comparison to the first experiment. There is also a slower growth in the required number of samples for the lower error threshold case as shown in Figure 6.9a. Figure 6.9b shows that the overall gap in runtime between the lower and higher accuracy thresholds is also significantly lower for this problem, requiring only as much as twice the time for the increased accuracy. Figure 6.10 verifies the expectation that the ranks at each level of the constructed hierarchical 


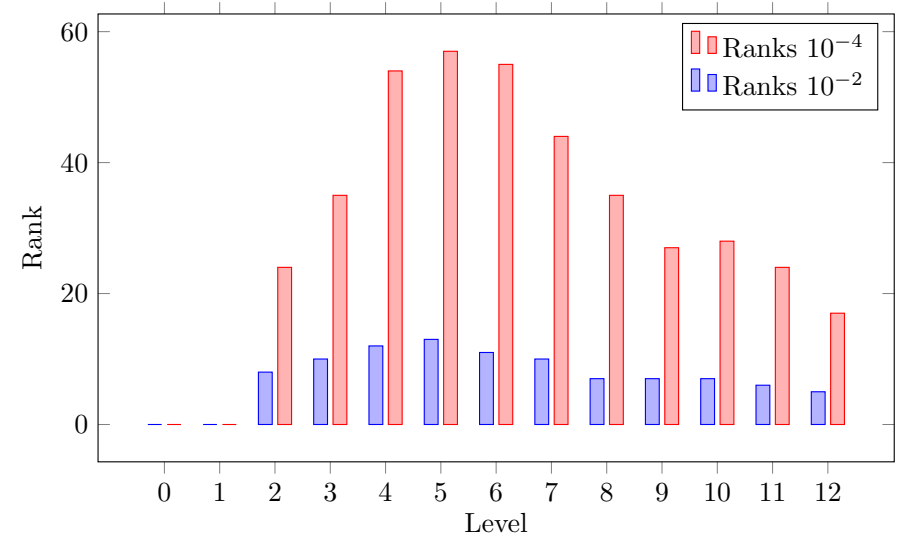

Figure 6.7: Comparison of the ranks per level for a problem size of $2^{18}$ for error thresholds of $10^{-2}$ and $10^{-4}$.

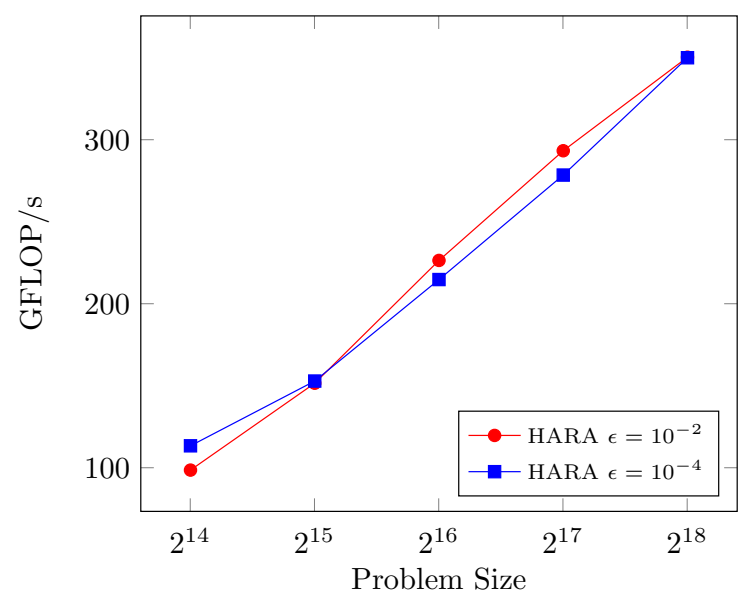

Figure 6.8: Overall performance for the construction of the hierarchical matrix on a P100 GPU.

matrix are also significantly reduced, leading to a heavier emphasis on the sampling phases for both thresholds. The accumulation and compression phases take up a smaller amount of the total computation as a result, as shown in Figure 6.11, and leads to greater overall performance for the higher accuracy as seen in Figure 6.12 due to the increased arithmetic intensity in the more efficient sampling phase. 


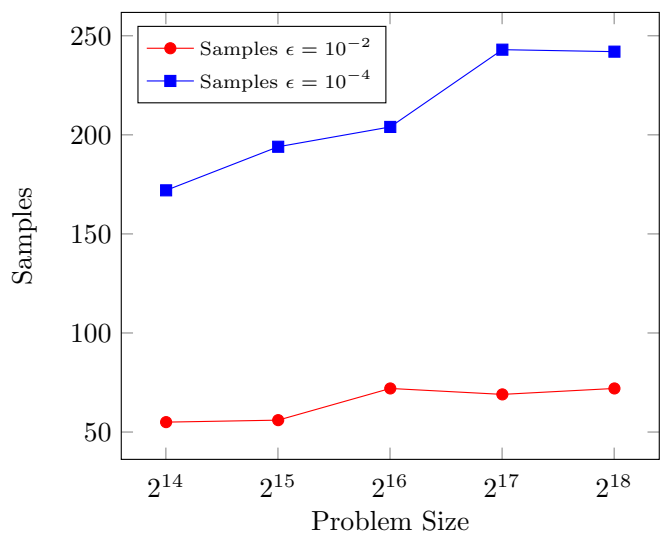

(a) Number of samples required.

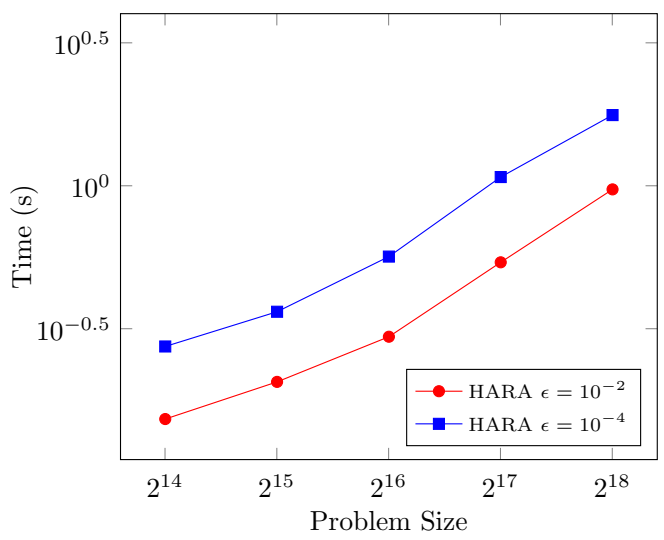

(b) GPU time for the construction.

Figure 6.9: Scalability and performance of HARA for constructing an $\mathcal{H}^{2}$ representation of $A^{2}$.

\subsubsection{Schur Complement Computation}

In this experiment, I show how HARA may be used to construct a Schur complement matrix, which is formally dense, directly as a hierarchical matrix. I consider a 5-pt (in 2D) and 7-pt (in 3D) discretizations of a constant coefficient Poisson operator on a uniform Cartesian grid, and partition the resulting sparse matrix as

$$
\left[\begin{array}{ll}
A_{11} & A_{12} \\
A_{21} & A_{22}
\end{array}\right]
$$

where "2" denotes the nodes that correspond to one boundary edge of the grid in 2D or boundary plane in $3 \mathrm{D}$, and " 1 " refers to the remaining nodes. I seek to compute the Schur complement of the 11 block. Computing Schur complements is a core computation in many factorization strategies, e.g., [83], and I plan to build on it in future work to develop direct sparse solvers for GPUs.

Given a factorization of $A_{11}=L_{11} L_{11}^{t}$ on the GPU, the cuSPARSE library can compute the product of the Schur complement $S=A_{22}-A_{12}\left(L_{11} L_{11}^{t}\right) \backslash A_{21}$ with vectors, via sparse matrix-vector products and sparse triangular forward and backward solves. This sequence of operations provides the black box sampling operation needed 


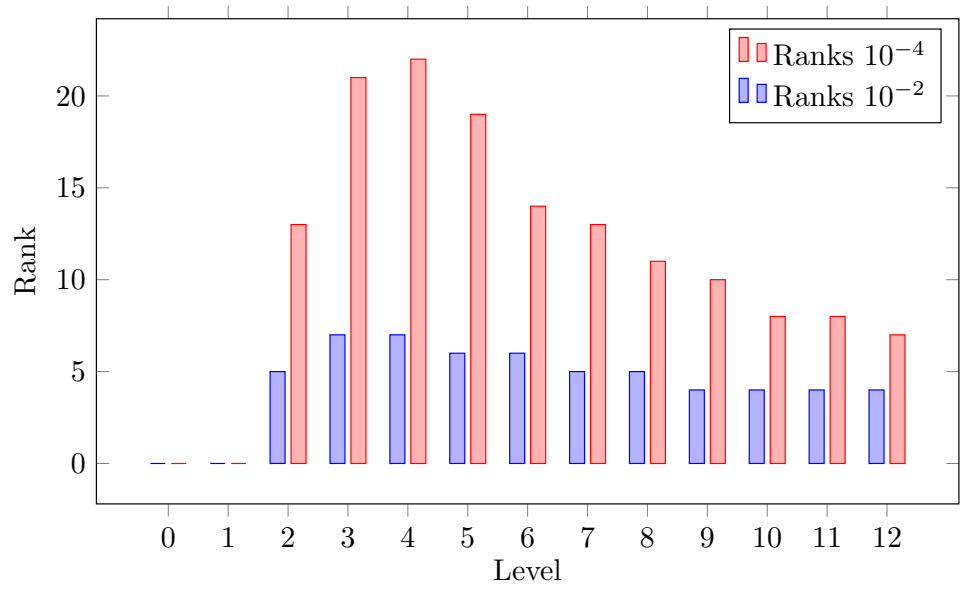

Figure 6.10: Comparison of the ranks per level when constructing the square of the matrix for a problem size of $2^{18}$ for error thresholds of $10^{-2}$ and $10^{-4}$.
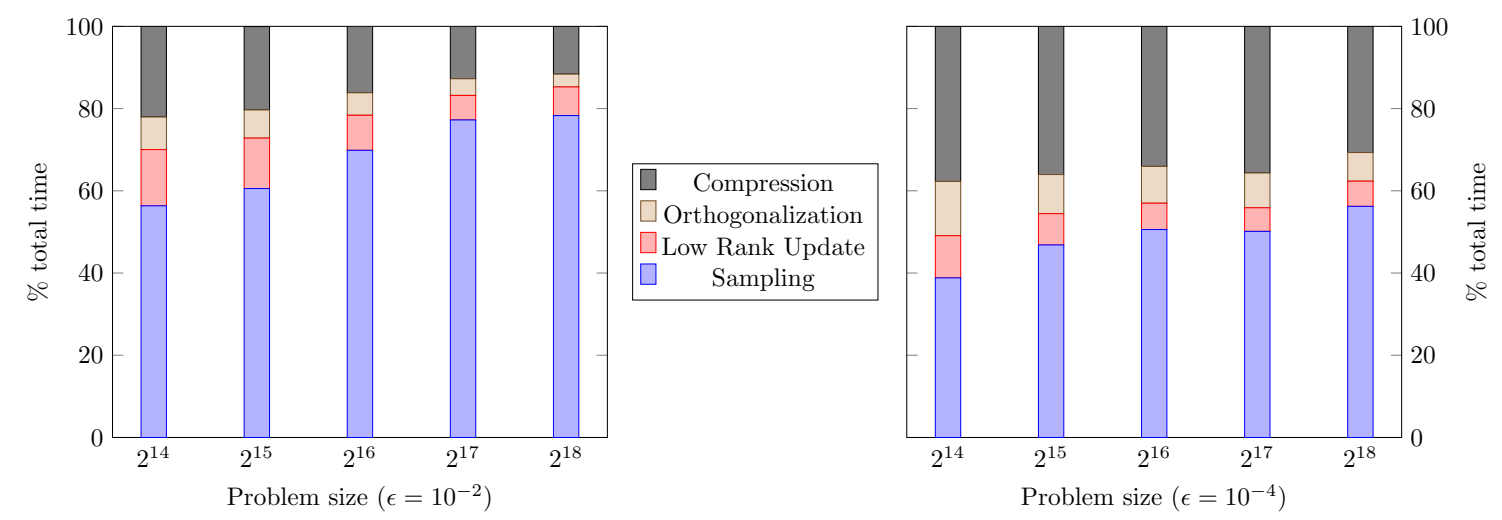

Figure 6.11: Profile showing relative costs of the different phases of the multiplication operation.

for the construction of $S$. For a $2 \mathrm{D} n \times n$ grid, the size of $S$ is $n \times n$ while the triangular solves are roughly of size $n^{2} \times n^{2}$. For a $3 \mathrm{D} n \times n \times n$ grid, the size of $S$ in $n^{2} \times n^{2}$ while the triangular solves are roughly of size $n^{3} \times n^{3}$. I use a weak admissibility structure for the approximation matrix in $2 \mathrm{D}$ and a slightly more refined structure with a standard admissibility condition for the 3D problem. All computations are done in double precision.

Table 6.1 lists the sampling and compression results of constructing the Schur complement for $2 \mathrm{D}$ and $3 \mathrm{D}$ problems of various sizes, to an accuracy of $\epsilon=10^{-4}$. 


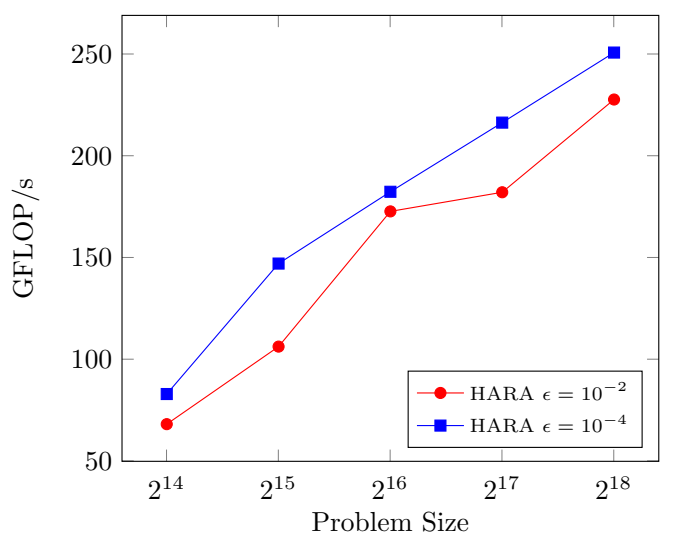

Figure 6.12: Overall performance for the construction of the square of an $\mathcal{H}^{2}$ matrix on a $\mathrm{P} 100$.

\begin{tabular}{|c|c|c|c|c|c|}
\hline Mesh Size & Size of S & $\begin{array}{c}\text { \# of } \\
\text { Samples }\end{array}$ & $\begin{array}{c}\text { Sampling } \\
\text { Time }(\mathrm{s})\end{array}$ & $\begin{array}{c}\text { Compression } \\
\text { Time }(\mathrm{s})\end{array}$ & $\begin{array}{c}\text { Memory (MB) / } \\
\text { Compression }\end{array}$ \\
\hline \hline $1024 \times 1024$ & $1024^{2}$ & 34 & 14.1 & 0.029 & $0.46 / 17.4$ \\
\hline $2048 \times 2048$ & $2048^{2}$ & 42 & 39.8 & 0.039 & $0.90 / 35.5$ \\
\hline \hline $32 \times 32 \times 32$ & $1024^{2}$ & 152 & 3.52 & 0.103 & $2.59 / 3.1$ \\
\hline $64 \times 64 \times 64$ & $4096^{2}$ & 397 & 140.7 & 0.817 & $20.0 / 6.4$ \\
\hline
\end{tabular}

Table 6.1: Results for computing the Schur complement to an accuracy of $\epsilon=10^{-4}$.

The sampling time dominates the total construction time, and more specifically the triangular solve portions of the sampling are the operations that dominate the total runtime, as triangular solvers are difficult to parallelize on the GPU. This bottleneck exists despite the fact that the triangular solvers of the cuSPARSE library benefit greatly from the increased arithmetic intensity and parallelism enabled by the blocking of the samples. For example, the sampling time was reduced to $14 s$ from $177 s$ for the 2D $1024 \times 1024$ problem, a speedup of over 12x over an unblocked, one-vector at a time, sampling.

As expected, the 2D problems have significantly smaller local ranks and therefore require fewer samples and show greater compression than the 3D problems, where compression is measured as the memory footprint of the full dense representation of $S$ (not constructed) over its hierarchical counterpart. In both cases the compression 
time without sampling, which includes generation and application of the low-rank updates, is a negligible portion of the total runtime. 


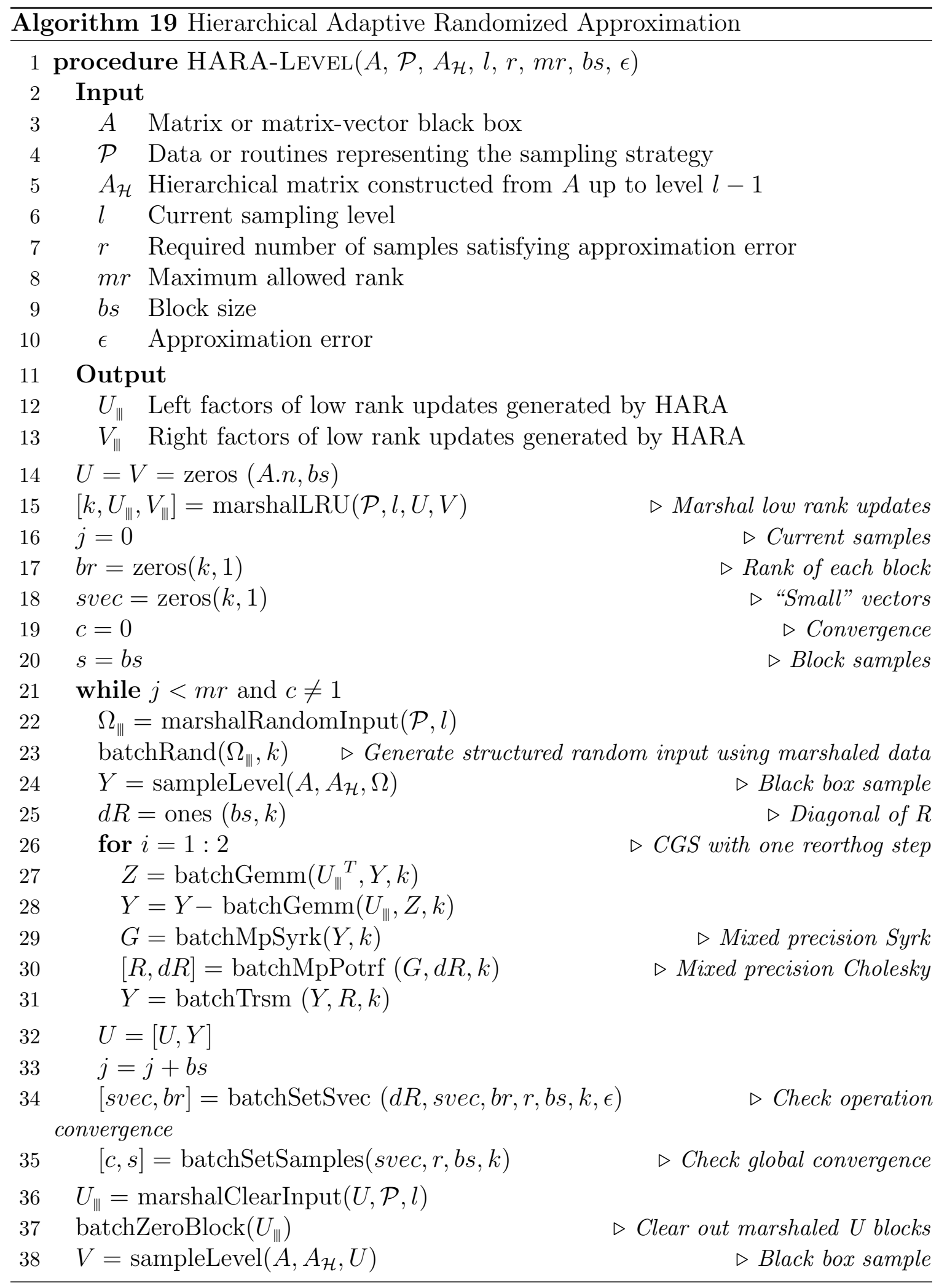



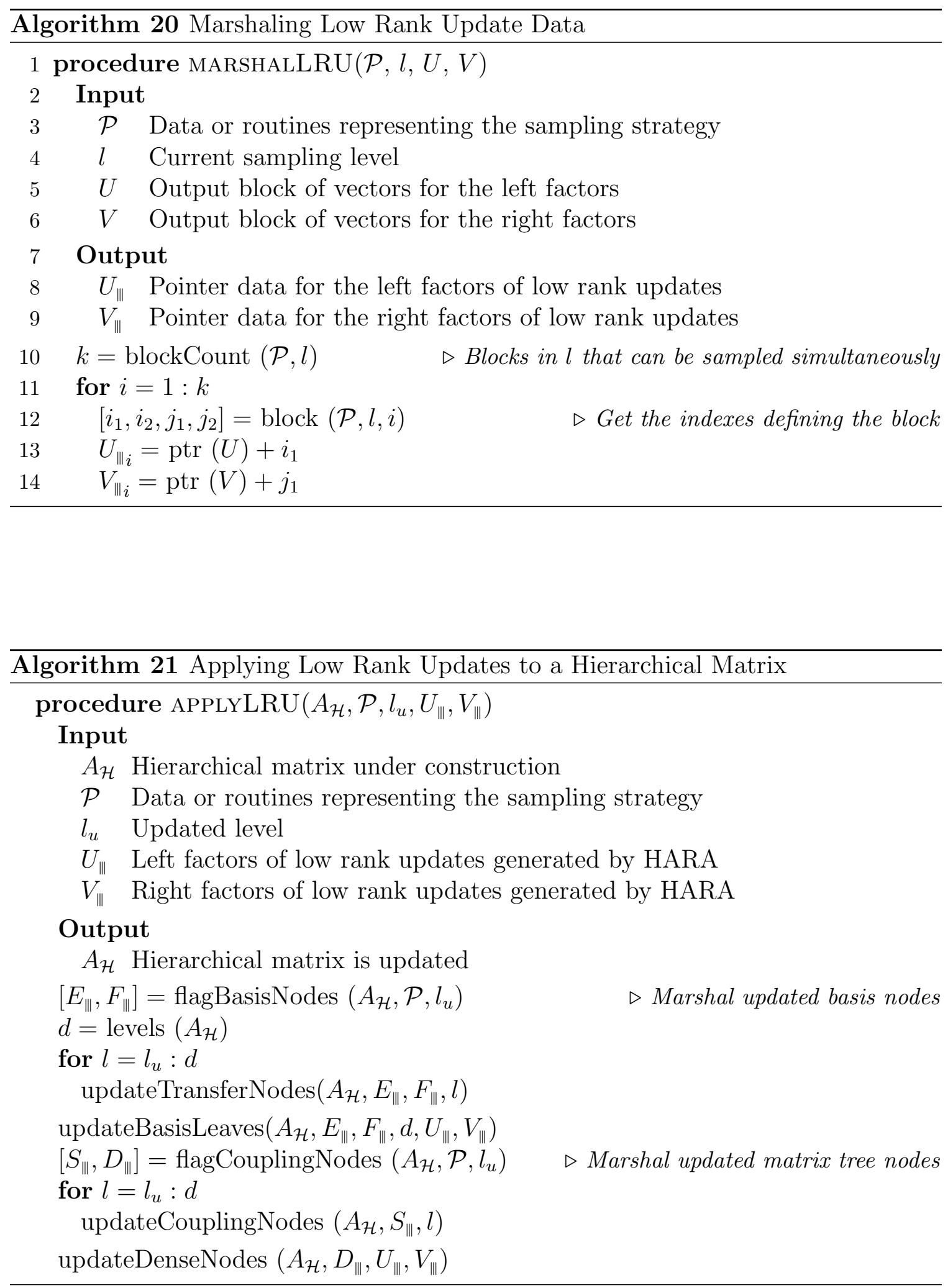


\section{Chapter 7}

\section{Iterative Methods for $\mathcal{H}^{2}$-matrix Inversion}

Iterative methods for computing the matrix inverse have a long history dating back to [84. Under reasonably general assumptions, they can be shown to be globally convergent methods, and require $2 \log \kappa+\log \log (1 / \epsilon)$ number of iterations to converge to an $\epsilon$ accuracy, where $\kappa$ is the condition number of the matrix being inverted [85].

Iterative inversion methods have not been very popular (but see [86]) because at face value their computational cost is larger than direct factorization-based inversion methods as they require two $O\left(n^{3}\right)$ matrix multiplications per iteration when used with dense storage. This calculus changes with hierarchical matrices, as the product of two such matrices may be obtained in log-linear time $O(k n \log n)$ [87]. This fast matrix multiply makes iterative inversion methods attractive here, even with memory considerations aside.

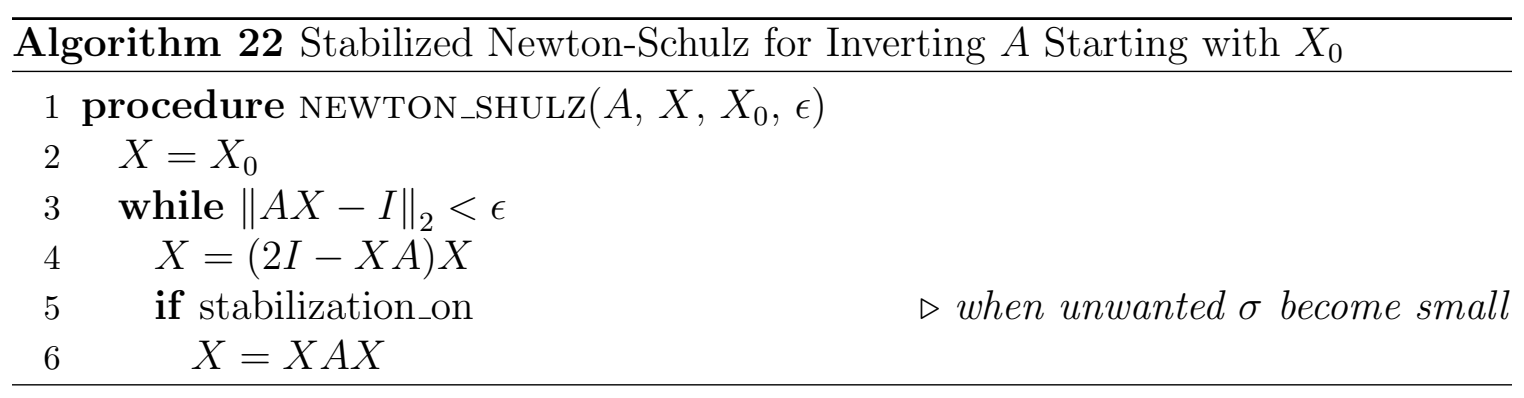

A basic stabilized Newton-Schulz (NS) iteration shown in Algorithm 22, For symmetric positive-definite matrices, a scaled identity $X_{0}=I /\|A\|_{\infty}$ may be used as the starting iterate and results in globally convergent iterations. Newton-Schulz converges only linearly in the early iterations before entering the rapid quadratic convergence 
regime. Its convergence can be sped up with improved initial guesses and convergence accelerations [88]. In an optimization context for example, the inverse from a previous iterate provides a natural starting point and this warm-start reduces the number of iterations compared to an agnostic starting point, as I illustrate below. The basic NS iteration is numerically stable and even self-correcting for nonsingular matrices. It may also be used to compute the Moore-Penrose pseudoinverse for singular matrices. However, for such matrices, and for matrices $A(\epsilon)$ where singular values below $\epsilon$ are ignored, it may be mildly unstable. The correction step in lines 5-6 of Algorithm 22 insures stability and need only be activated after the unwanted singular values of $X A$ are small enough, a condition that can be inexpensively monitored [88].

\subsection{Newton-Schulz Iterations with Hierarchical Matrices}

To adapt iterative methods to the hierarchical matrix representation context, two modifications are needed as shown in Algorithm 23. The first comes from the fact that the primary fast operation, especially on GPUs, is a blocked matrix-vector multi-

plication; the construction of the Schulz iterates is done via the procedure of Chapter 6 where a sampler, i.e., a matrix-vector expression evaluator, is provided to the construction procedure at every iteration. For example, a simple sampler for NS would follow Algorithm 24 to produce the samples $Y$ from random input vectors.

The second, more consequential modification is that the iterative algorithm may be carried out with truncation - an option made possible by the tunable-accuracy nature of the hierarchical representation. As solving exactly the tangent system in the early phases of the Newton method is a waste of computational resources, it makes little sense to construct the matrices in the first iterations to the ultimately desired accuracy. In fact, Hackbusch et al. [89] showed that, under fairly general conditions, the intermediate iterates $X_{k}$ may be replaced by approximations without affecting the convergence rate of the method. Therefore I have the freedom to choose the accuracy 
$\epsilon_{k}$ to which the intermediate iterates are to be generated. A more effective strategy starts with a low accuracy in the early iterations and gradually reduces it as convergence is approached. As I show below, the use of such a dynamic threshold reduces substantially the overall computational cost of inversion by producing intermediate iterates with smaller footprints.
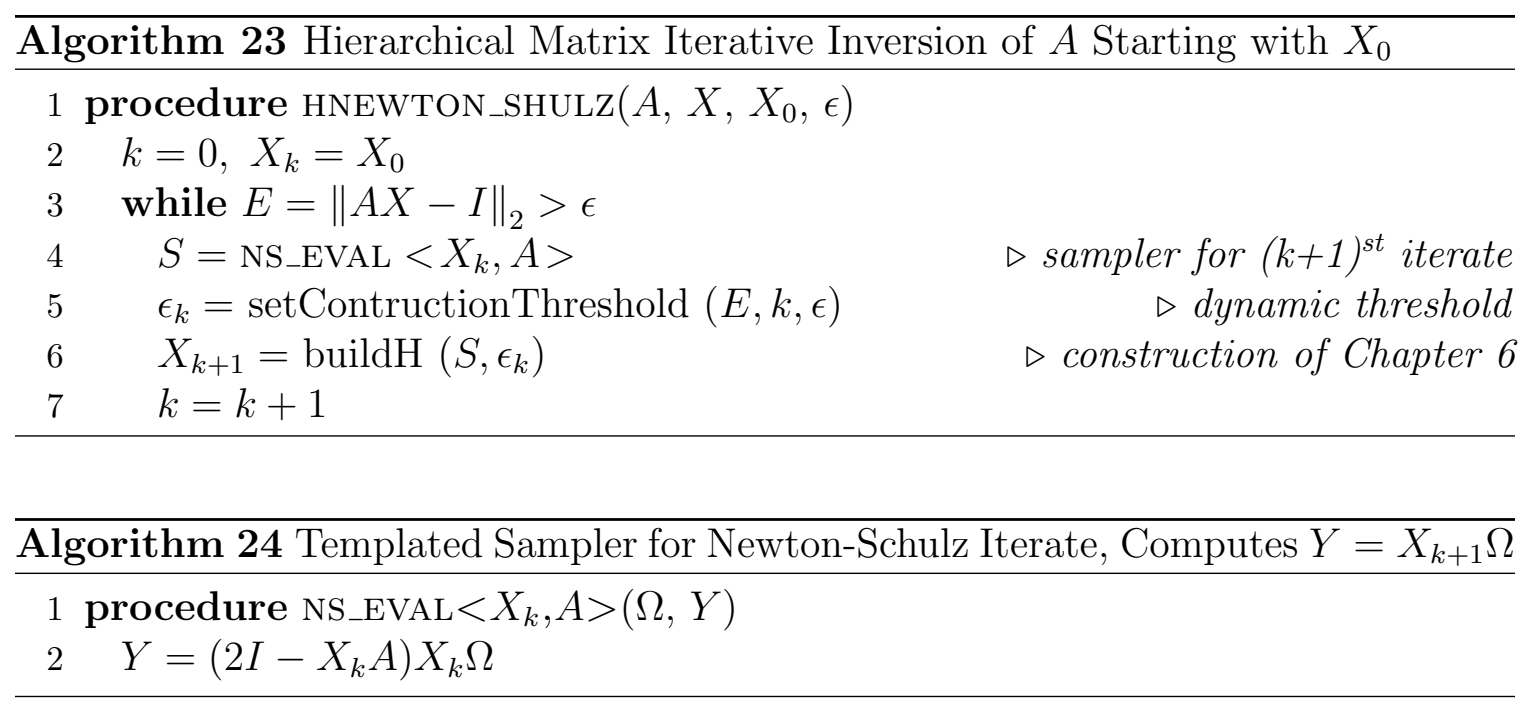

I show the effectiveness of Algorithm 23 on the following minimal surface problem. Let $m(x)$ be the height of a surface defined in the unit square $\Omega=[0,1]^{2}$.

$$
\begin{array}{ll}
\underset{m(x)}{\operatorname{minimize}} & J(m)=\int_{\Omega} \sqrt{1+|\nabla m|^{2}} d \Omega, \\
\text { subject to } & m(\partial \Omega)=m_{0} .
\end{array}
$$

I discretize $m(x)$ and the objective using finite differences on an $n=128 \times 128$ grid. The Hessian for this problem can be computed exactly, up to discretization errors, since it corresponds to the linearization of a nonlinear Poisson equation. Its inverse, however, is dense, and therefore it is a good test for the effectiveness of the iterative inversion method described above, since all resulting approximation errors are attributable to it.

For the following experiments, I set the compression threshold used during the construction of the hierarchical matrix to $\epsilon_{k}=10^{-6}$ with a leaf size of 64 . Starting 
from the scaled identity $X_{0}=\frac{I}{\|A\|_{\infty}}$, inverting the Hessian using NS for the first optimization step needs quite a few iterations, with the number of required samples for intermediate iterates increasing very rapidly before receding as it converges as shown in Figure 7.1a. The total time needed to invert the Hessian using this method is about 178 seconds on a P100 GPU, with over $75 \%$ of the total runtime spent in compression. To alleviate the impact of the intermediate iterates, I can start with a relatively loose compression error threshold $\epsilon_{k}$, tightening the threshold as converge is approached. This significantly reduces the number of samples needed for the earlier iterations as shown in Figure 7.1a, where I start with a much looser threshold of $10^{-2}$. The total runtime is then reduced to $51 \mathrm{~s}$, a 3.5 fold reduction in inversion time, with $69 \%$ of the time spent in compression.

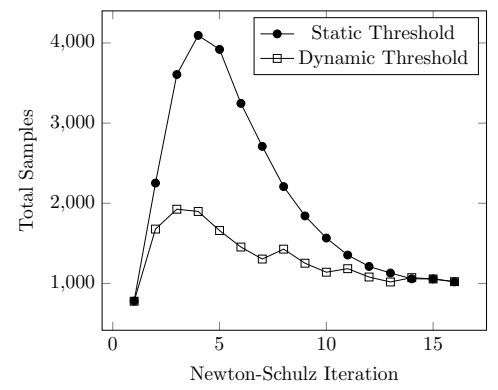

(a)

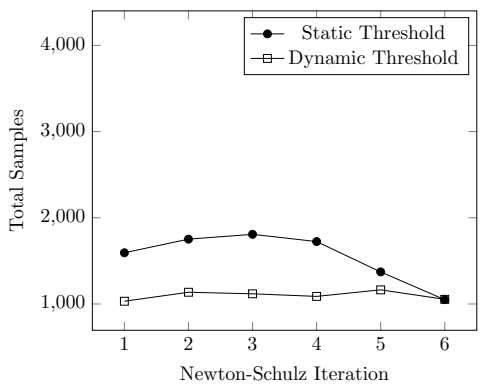

(b)

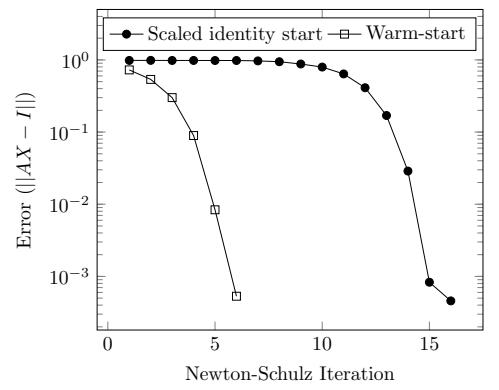

(c)

Figure 7.1: Performance of Hessian inversion on a problem of size $n=16,384$. Number of Hessian-vector products throughout the NS iterations: (a) starting from a scaled identity and (b) a warm starting from previous iteration. (c) Convergence history.

From the second optimization iteration onwards, I can use the approximate Hessian inverse from the previous iteration as an initial guess, allowing NS to converge in fewer iterations. While the first optimization iteration converged in 16 NS iterations, the second iteration needed only 6 , inverting the Hessian in 32s if a static threshold is used and in $14 \mathrm{~s}$ with a dynamic threshold. In Figure $7.1 \mathrm{~b}$, I compare the number of needed samples for static and dynamic thresholds. Finally, the error in the inverse $\|A X-I\|_{2}$ throughout the NS procedure is shown in Figure $7.1 \mathrm{c}$ for a given optimization step, exhibiting rapid convergence as I approach the solution. Warm- 
starting from previous iterations results in the iterations entering the fast convergence regime earlier.

\subsection{Higher Order Methods for Faster Convergence}

The results above show that the hierarchical matrix compression portion of the construction algorithm dominates the total runtime of the inversion algorithm. This is because I apply the low rank updates generated during the sampling phase in relatively small blocks due to memory constraints of the GPU. The majority of the resulting linear algebra operations during compression, such as batched rank-revealing QR and tall skinny QR decompositions, are not particularly efficient on GPUs and are relatively costly. On the other hand, the sampling operations, which primarily consist of batched matrix-matrix products and blocked sparse matrix vector products, are highly parallel, efficient, and arithmetically intensive. Taking this disparity into consideration, I employ high order hyperpower iterative methods to shift the computational load to the sampling phase. An order $l$ hyperpower iteration is defined as 90]

$$
X_{k+1}=X_{k}\left(I+R_{k}+\cdots+R_{k}^{l-1}\right)=X_{k} \sum_{i=0}^{l-1} R_{k}^{i}
$$

where $R_{k}=I-A X_{k}$, involving $l$ matrix products. Setting $l=2$ gives me the standard NS iteration of the previous section. Most previous work on efficient hyperpower iterations with dense matrices attempts to reduce the number of matrix-matrix products by calculating a few temporary matrices and factoring the summation in 7.2 . Here, I seek to avoid the costly compression for those temporary matrices, and use the original form of the equation which performs $l$ products. This achieves the goal of concentrating the workload on the far more efficient sampling phase of the computation, and achieves considerable time savings. Sampling $X_{k+1}$ can be done efficiently using a method similar to Horner's method for polynomial evaluation, as shown in 
Algorithm 25 which replaces the NS evaluation of line 4 of Algorithm 23.

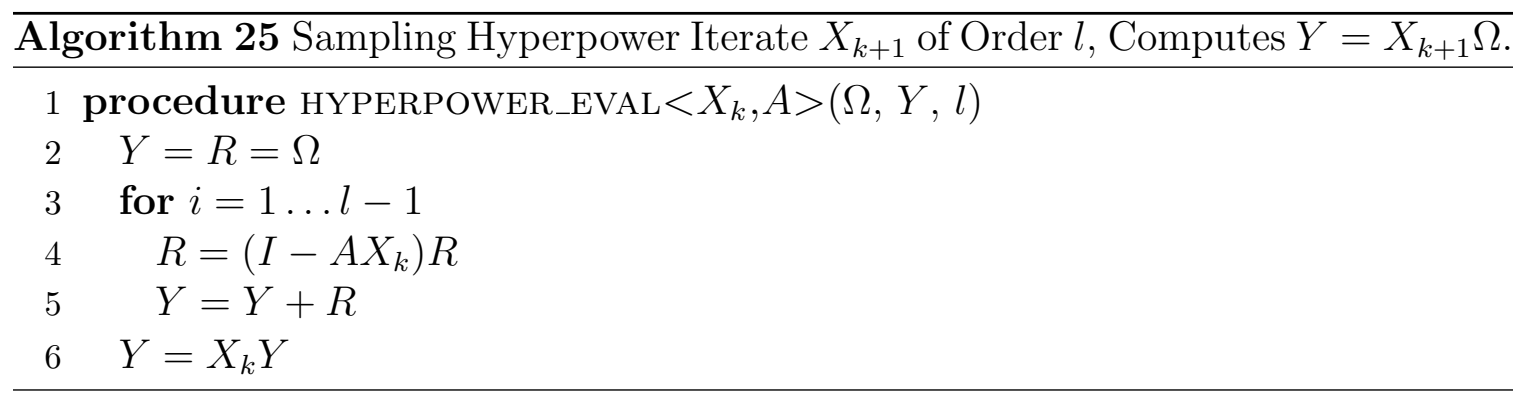

The performance of higher order methods is illustrated in Figure $7.2 \mathrm{a}$ which shows the number of iterations and the samples taken in each iteration for hyperpower iterations of order $l=8,16,32$, which are notably lower than those of Figure 7.1a. High order methods also have the benefit of faster convergence as shown in Figure $7.2 \mathrm{~b}$ where the order 8 method takes 7 iterations to converge and the order 16 and 32 methods take 6 iterations, as opposed to the 16 iterations needed by the second order method. The overall inversion times are also considerably lower, with order 8 and 16 at 20 seconds and the order 32 at 25s. The order 8 method does one more iteration than the order 16 method, but the lower sampling cost puts it on equal footing, whereas the order 32 performs the same number of iterations as the order 16 method while having higher cost per sample.

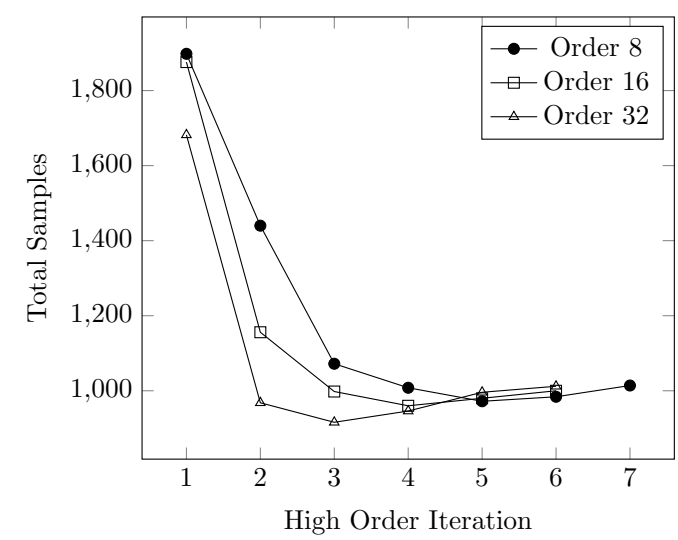

(a) Number of samples vs iterations.

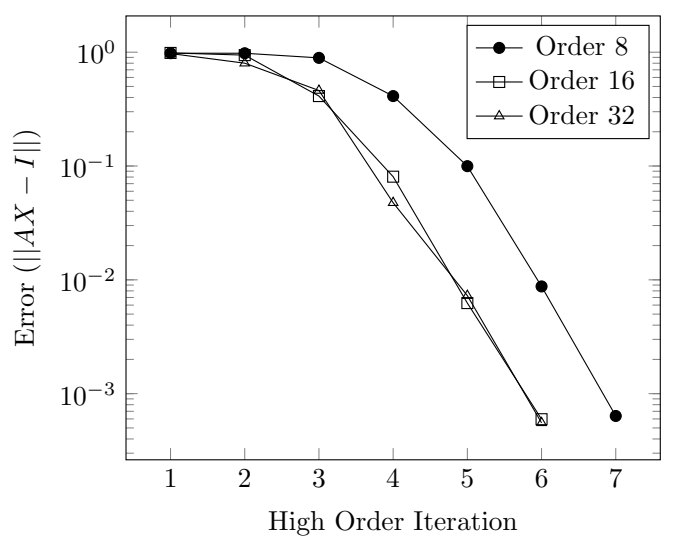

(b) Convergence history.

Figure 7.2: Performance of Hessian inversion using high order iterations of order 8, 16 , and 32 . 


\subsection{Unrolling Iterations}

As another algorithmic optimization for the example problem (7.1), I consider the effect of loop unrolling. The low number of NS iterations needed for the second optimization iteration onwards, due to the use of the previous approximate Hessian inverse as the initial NS iterate, presents another opportunity for greater inversion performance. By unrolling the iterates to express $X_{k}$ in terms of $X_{0}$, I can achieve the same goal as the higher order methods, where the workload is shifted to the sampling phase and the increase in samples for the intermediate iterates can be avoided; the $k$-th unrolled iterate can be evaluated as the hyperpower iterates as:

$$
\begin{aligned}
X_{k} & =X_{k-1}\left(2 I+A X_{k-1}\right) \\
& =X_{k-2}\left(2 I+A X_{k-2}\right)\left(2 I+A X_{k-2}\left(2 I+A X_{k-2}\right)\right)=\ldots \\
& =X_{0} \sum_{i=0}^{2^{k}}\left(\begin{array}{c}
2^{k} \\
i
\end{array}\right)\left(-A X_{0}\right)^{i} .
\end{aligned}
$$

Since the number of entries in the sum grows exponentially, I can efficiently unroll only a small number of iterations (say 5 or 6 ); however, since the number of iterations needed after the first optimization iteration is small, I can effectively unroll all of the required iterations, and obtain the approximate inverse in a single construction. This further reduces the inversion time from 14 seconds to 4 seconds, giving me another $3.5 \times$ improvement on the dynamic error threshold inversion.

The inversion methods introduced in this chapter demonstrate the performance benefits of concentrating the computational effort in the arithmetically intensive sampling phase, not only in terms of reducing inversion time as in the unrolled iterations, but also in terms of reducing the total number of iterations required for convergence, as in the higher order methods. Related methods for computing square roots and inverse square roots may be similarly formulated and optimized. 


\section{Chapter 8}

\section{Distributed Hierarchical Matrix Operations}

Building on the previously developed routines, I demonstrate the scalability of the hierarchical matrix operations when distributed over multiple GPUs connected by a network or on the same node. Due to the structural similarity of most of the operations discussed in this thesis, where upsweep and downsweep operations are carried out on the basis trees and multiplication and projection operations are performed on the matrix trees using the byproducts of those sweeps, I tackle the problem of carrying out a hierarchical matrix vector multiplication of a distributed $\mathcal{H}^{2}$-matrix with multiple vectors. I show how the matrix is constructed in its distributed form and discuss the details of an initial distributed hgemv operation, where I show that a naive implementation will not scale well. Finally, I improve scalability by optimizing communication as well as hiding communication and GPU specific overheads. Table 8.1 summarizes the symbols used in the description of the distributed algorithms of this chapter.

\subsection{Distributed Construction}

While the discussion in this section is limited to the decomposition of symmetric hierarchical matrices, i.e., matrices where the row and column bases are the same, the methods can be easily generalized to the non-symmetric case. Treating each level of the matrix tree of the hierarchical matrix as a block sparse matrix, the aim for the symmetric case is to decompose the levels into block rows, with each block row stored 


\begin{tabular}{ll}
\hline Symbol & Description \\
\cline { 1 - 1 }$P$ & Data set of all discretization particles \\
$p$ & Total number of GPUs \\
$\mathcal{U}$ & Index of the local GPU \\
$\mathcal{A}$ & Complete basis tree \\
${ }_{x}, y$ & Complete matrix tree \\
${ }_{p} n$ & Input and output vectors $x$ and $y$ \\
${ }_{p} \mathcal{U}$ & Nocal branch of the basis tree on GPU $p$ \\
${ }_{r} \mathcal{U}$ & Root branch of the basis tree on the master process \\
${ }_{p} \mathcal{A}$ & Local branch of the matrix tree on GPU $p$ \\
${ }_{r} \mathcal{A}$ & Root branch of the matrix tree on the master process \\
${ }_{p} \widehat{x},{ }_{p} \widehat{y}$ & Local branch of $\widehat{x}$ and $\widehat{y}$ on GPU $p$ \\
${ }_{p} x,{ }_{p} y$ & Local sub-vector of the input $x$ and output $y$ on GPU $p$ \\
${ }_{p} \mathcal{A}_{q}$ & Matrix tree branch due to the dual tree traversal of ${ }_{p} \mathcal{U}$ and ${ }_{q} \mathcal{U}$ \\
\hline
\end{tabular}

Table 8.1: Notation used for distributed hierarchical matrix elements.

on a single GPU. The basis tree is similarly decomposed by level, assigning the nodes corresponding to the stored block rows to the same GPU. This decomposition allows much of the upsweep and downsweep operations to be carried out independently as described in Section 8.2 and is illustrated in Figure 8.1. It does however present some challenges to the matrix tree operations, such as the projection phase of hcompress and multiplication phase of hgemv. In Section 8.3, I present optimizations, taking advantage of the properties of hierarchical matrices, to overcome these challenges.

Starting the construction of the distributed matrix in the same way as the single GPU method, I hierarchically partition the index set of rows and columns of the matrix $\mathcal{I}$ and $\mathcal{J}$ to produce cluster trees $T_{\mathcal{I}}$ and $T_{\mathcal{J}}$. Without any loss of generality, I assume that I have a symmetric matrix and $T_{\mathcal{I}}=T_{\mathcal{J}}$. $T_{\mathcal{I}}$ is split into $P$ independent branches at level $l=\log (P)+1$ and a local branch ${ }_{p} \mathcal{U}$ of the basis tree on GPU $p$ is generated for each $p \in\{0 \ldots P-1\}$. The top $l$ levels of the basis tree are kept on a master process and could potentially be recursively split further if $P$ becomes large enough. To simplify the discussion, I will assume that the top levels are kept entirely on a single master process as a single root branch ${ }_{r} \mathcal{U}$. Note that the nodes 


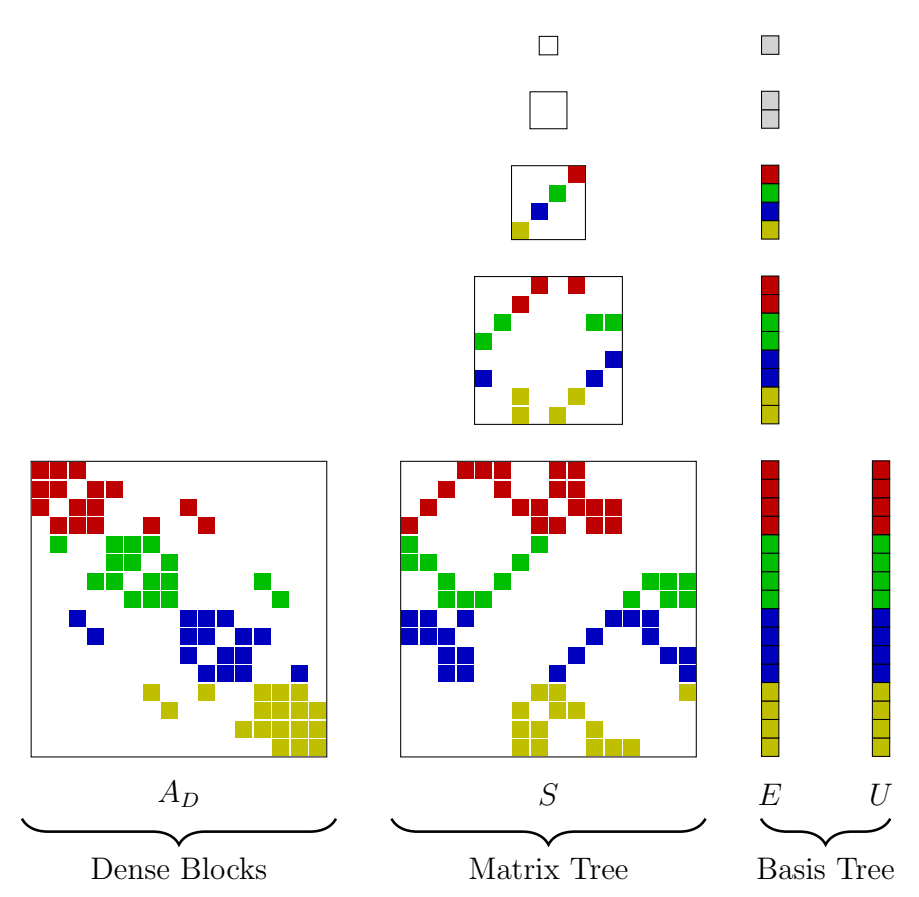

Figure 8.1: A hierarchical matrix distributed to 4 GPUs with the block rows of the dense and coupling leaves having the same color residing on the same GPU. The root branch is colored in grey.

of the leaf level of ${ }_{r} \mathcal{U}$ are duplicated as the roots of each of the branch trees ${ }_{r} \mathcal{U}$ to allow upsweep and downsweep operations to begin and end at those root nodes on the master process. The root branch of the matrix tree ${ }_{r} \mathcal{A}$ can be generated on the master process in any number of ways, such as the general admissibility dual tree traversal Algorithm 26 on the root node of ${ }_{r} \mathcal{U}$. Once this matrix tree is constructed, a list of the basis nodes $L_{p}$ from ${ }_{r} \mathcal{U}$ can be extracted for each GPU $p$ corresponding to the inadmissible nodes of the block row $p$ at level $l-1$ of the matrix tree using Algorithm 27. Once the list for each process has been compiled, they are scattered from the master process to all other processes.

Since the nodes $q$ in $L_{p}$ were generated from the inadmissible nodes of the matrix tree $A_{p q}^{l-1}$ that have to be subdivided further, the structure of the matrix tree ${ }_{p} \mathcal{A}$ on each GPU $p$ can then be generated independently using multiple dual tree traversals of the root node of ${ }_{p} \mathcal{U}$ with the nodes $q$ in $L_{p}$, where each traversal generates a local branch ${ }_{p} \mathcal{A}_{q}$. Algorithm 28 shows the overall procedure for the construction of 

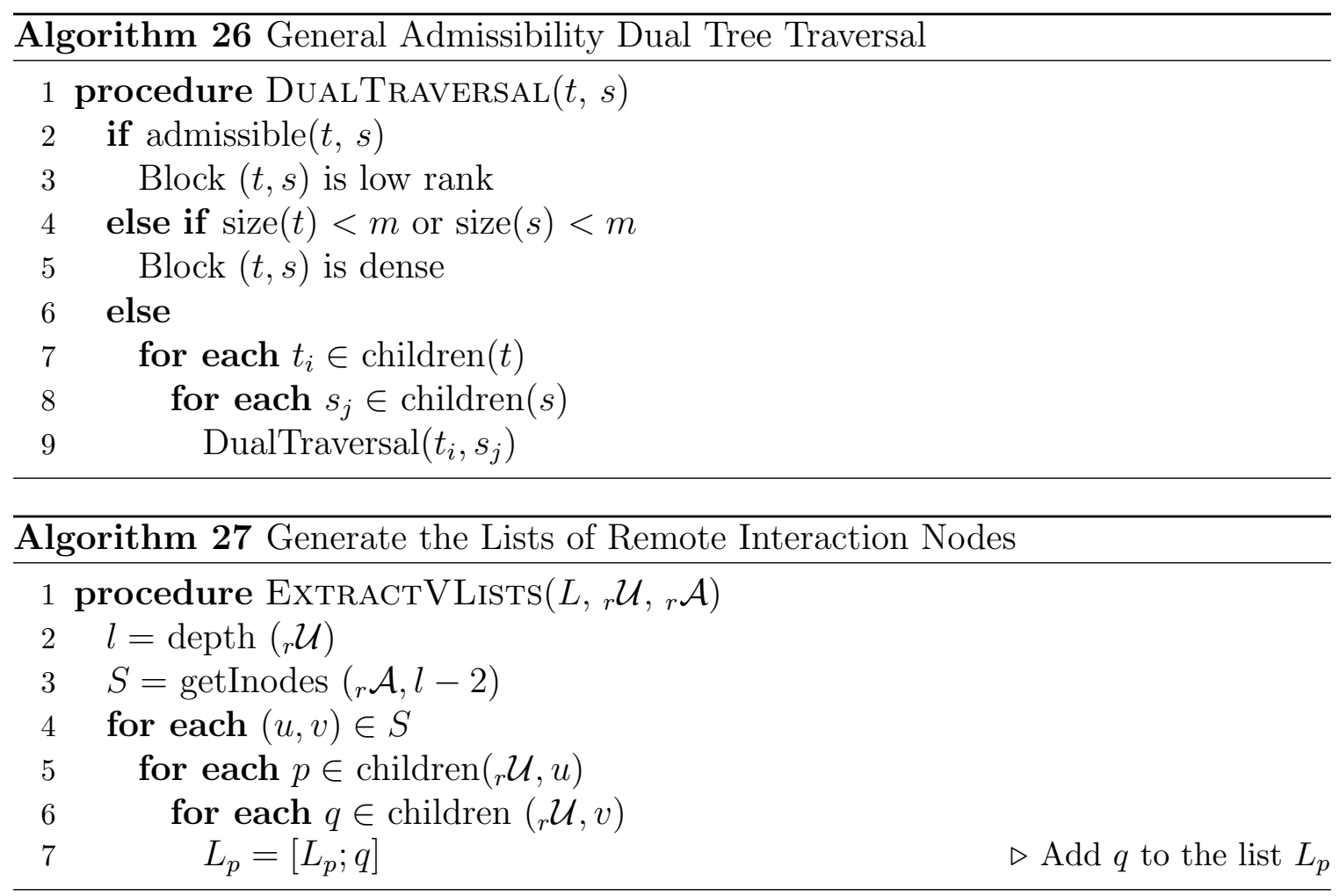

the structure of the distributed hierarchical matrix. Once the structure has been determined, the entries of the transfer and leaf matrices of the basis tree and the coupling and dense matrices of the matrix tree can be populated independently on each GPU.

\subsection{Distributed Hierarchical Matrix Vector Products}

This section describes the details of an initial implementation of the product of a distributed hierarchical matrix with $b s$ vectors, highlighting the challenges that the chosen decomposition pose when it comes to communication among processes. The input vector $x$ is distributed among the $P$ GPUs, where each sub-vector ${ }_{p} x$ is extracted from the index set defined by the root cluster node of ${ }_{p} \mathcal{U}$. The distributed hgemv follows the same four phases as the single GPU hgemv: an upsweep, coupling multiplication, a downsweep and a dense multiplication phase. 

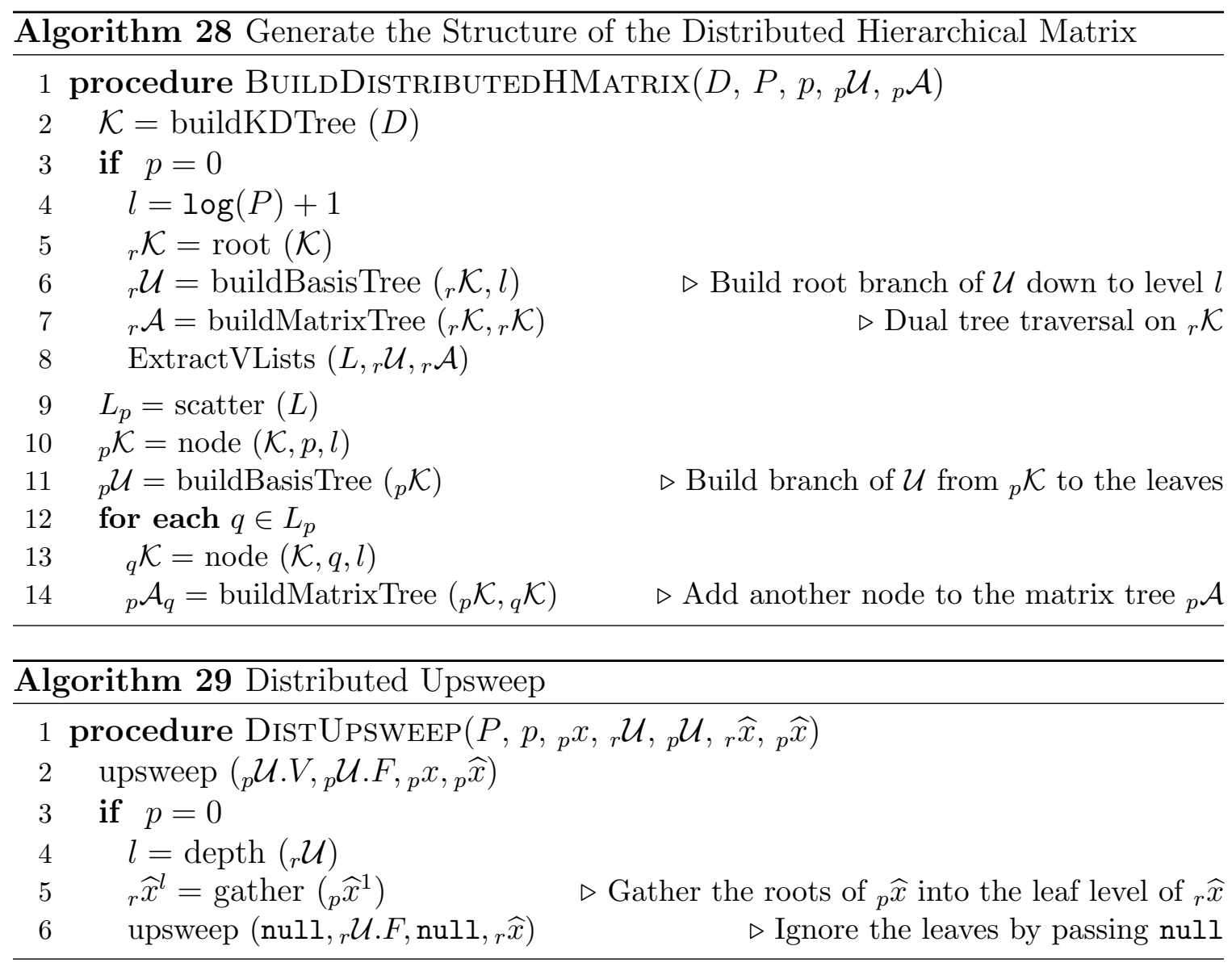

\subsubsection{Distributed Upsweep}

The upsweep on each GPU $p$ can proceed in parallel on each of the local branches ${ }_{p} \mathcal{U}$. Once the upsweep reaches the roots of each branch, the root branch ${ }_{r} \mathcal{U}$ can proceed by issuing a gather operation on the master process of all the root nodes of the ${ }_{p} \widehat{x}$ trees generated by the branch upsweeps. This will populate the leaf level of the root branch ${ }_{r} \widehat{x}$ on the master process, allowing the upsweep to proceed to the root. The regular upsweep Algorithm 9 can be used for the branch upsweep as well as the root upsweep simply by ignoring the first batched operation on the leaves of the root branch, since that step is technically replaced by the gather operation. The distributed upsweep is presented in Algorithm 29. 


\subsubsection{Distributed Multiplication}

In Section 8.1, the matrix tree is distributed by interpreting each level of the tree as a block sparse matrix and using a block row decomposition of each level. Since the block row local to a GPU can require input from $\widehat{x}$ nodes belonging to a remote GPU, an allgather on ${ }_{p} \widehat{x}$ can assemble all branches of $\widehat{x}$ on all GPUs. Once assembled, the multiplication phase can proceed on each GPU independently on the coupling matrices of ${ }_{p} \mathcal{A}$ using the TreeMultiply routine of Algorithm 10. Processing the multiplication of the root branch ${ }_{r} \mathcal{A}$ on the master process to produce the root branch ${ }_{r} \widehat{y}$ finalizes the phase as shown in Algorithm 30. The dense multiplication follows the same method, with an allgather on the input sub-vectors ${ }_{p} x$ to produce the output sub-vector ${ }_{p} y$.

\subsubsection{Distributed Downsweep}

After the local branch ${ }_{p} \widehat{y}$ is generated on each GPU and the root branch ${ }_{r} \widehat{y}$ is completed on the master process, the distributed downsweep can proceed, starting at the root of ${ }_{r} \widehat{y}$ and following Algorithm 11. Since the root branch has no leaves, that phase can be skipped in the same way as the leaves during the upsweep. After the root downsweep is done and the leaf level of ${ }_{r} \widehat{y}$ has been updated, it can be scattered from the master process to all other GPUs and added to the roots of ${ }_{p} \widehat{y}$. The addition is necessary since unlike the upsweep operation, the root nodes of ${ }_{p} \widehat{y}$ contain data from the previous multiplication phase. Finally, the downsweeps on each GPU can proceed independently on each local branch to update all nodes of ${ }_{p} \widehat{y}$ down to the leaf level

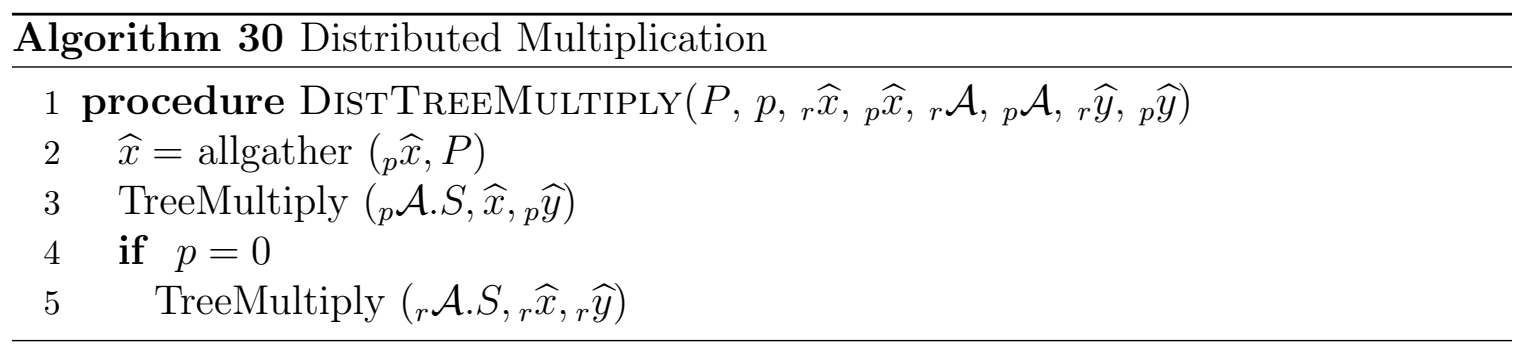




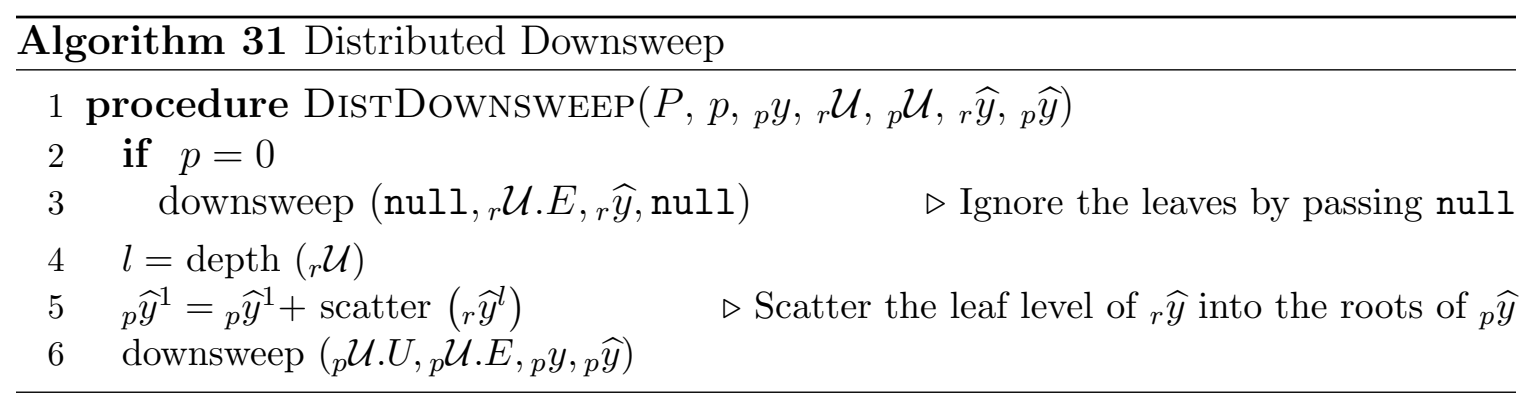

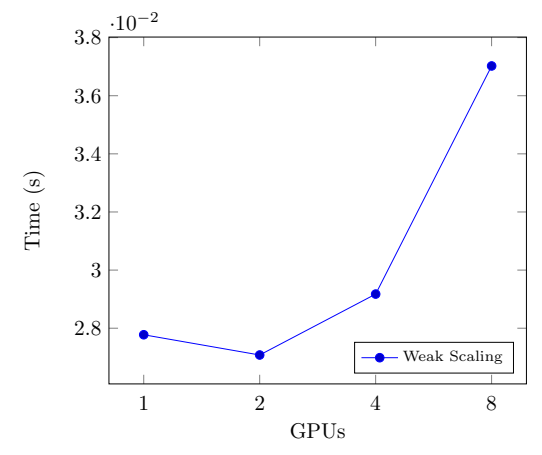

(a) Weak scaling behaviour on 8 K80 GPUs with $n_{p}=2^{20}$ per GPU.

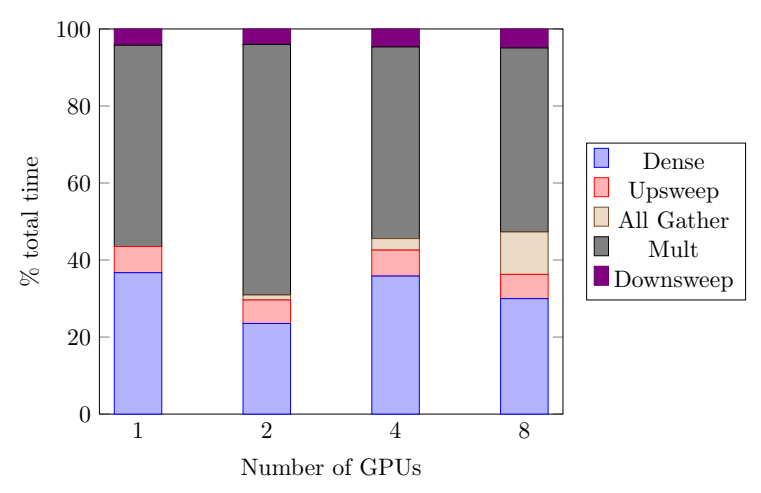

(b) The profile of the distributed hgemv using allgather.

Figure 8.2: The weak scaling and the profile of the distributed hgemv.

and into the output sub-vector ${ }_{p} y$ as show in Algorithm 31 .

\subsubsection{Communication Overhead}

While the upsweep and downsweep have fairly reasonable communication costs, the allgather of the input vector $x$ and $\widehat{x}$ branches during the multiplication phase leads to an $O(n)$ growth in memory consumption and execution time that will eventually dominate the computation. To demonstrate this, I study the weak scaling behaviour of the algorithm on 8 K80 GPUs, keeping the problem size at $n_{p}=2^{20}$ per GPU. The poor weak scaling can be seen in Figure $8.2 \mathrm{a}$ due to the steadily increasing allgather portion of the computation as shown in Figure $8.2 \mathrm{~b}$. To get closer to the ideal scaling, this communication overhead is reduced in the next section. 


\subsection{Optimizing Communication Volume}

One useful property of hierarchical matrices is that for many types of problems, the sparsity constant $C_{s p}$, i.e., the maximum number of blocks in any block row or column at any level of the matrix tree, is bounded independently of the problem size [9]. In a distributed setting, this means that only a limited number of $\widehat{x}$ nodes interact with the coupling nodes of the matrix tree in a given block row. In particular, the number of $\widehat{x}$ nodes that interact with the off-diagonal portion of a block row is bounded. Since the diagonal block of the matrix tree interacts with the local branch ${ }_{p} \widehat{x}$ on GPU $p$, only a limited number of nodes from remote branches ${ }_{q} \widehat{x}$ need to be sent per process. The same logic applies to the dense blocks within the block row and the input vector $x$. In this section, I will determine the nodes that have to be sent, describe a marshalling routine that packs those nodes into a single buffer, and discuss some complications that can arise when sending and receiving the buffers on GPUs.

\subsubsection{Compressing the Off-diagonal Blocks}

Since only the basis node data that interacts with the off-diagonal portion of ${ }_{p} \mathcal{A}$ must be compressed, the matrix tree is first split into two distinct trees: a diagonal matrix tree ${ }_{p} \mathcal{A}_{p}$ and an off-diagonal matrix tree ${ }_{p} \mathcal{A}_{\bar{p}}$. While the off-diagonal portion could simply be represented as a set of trees, with one tree for every interaction ${ }_{p} \mathcal{A}_{q}$ with a GPU $q$, it is far more efficient to have them all in a single flattened tree to allow any marshaling operations on the off-diagonal blocks to be completed with a single kernel call. To keep track of the GPU $q$ that a particular node belongs to, an array of boundary node indices limits is needed, where GPU $q$ will hold nodes limits $[q]$ to $\operatorname{limits}[q+1]$.

Once the trees are split, the list of basis nodes that interact with ${ }_{p} \mathcal{A}_{\bar{p}}$ can be generated by iterating through the $(t, s)$ pairs of each coupling node in the tree and determining all unique $s$ values by inserting them into a map smap. This map will 


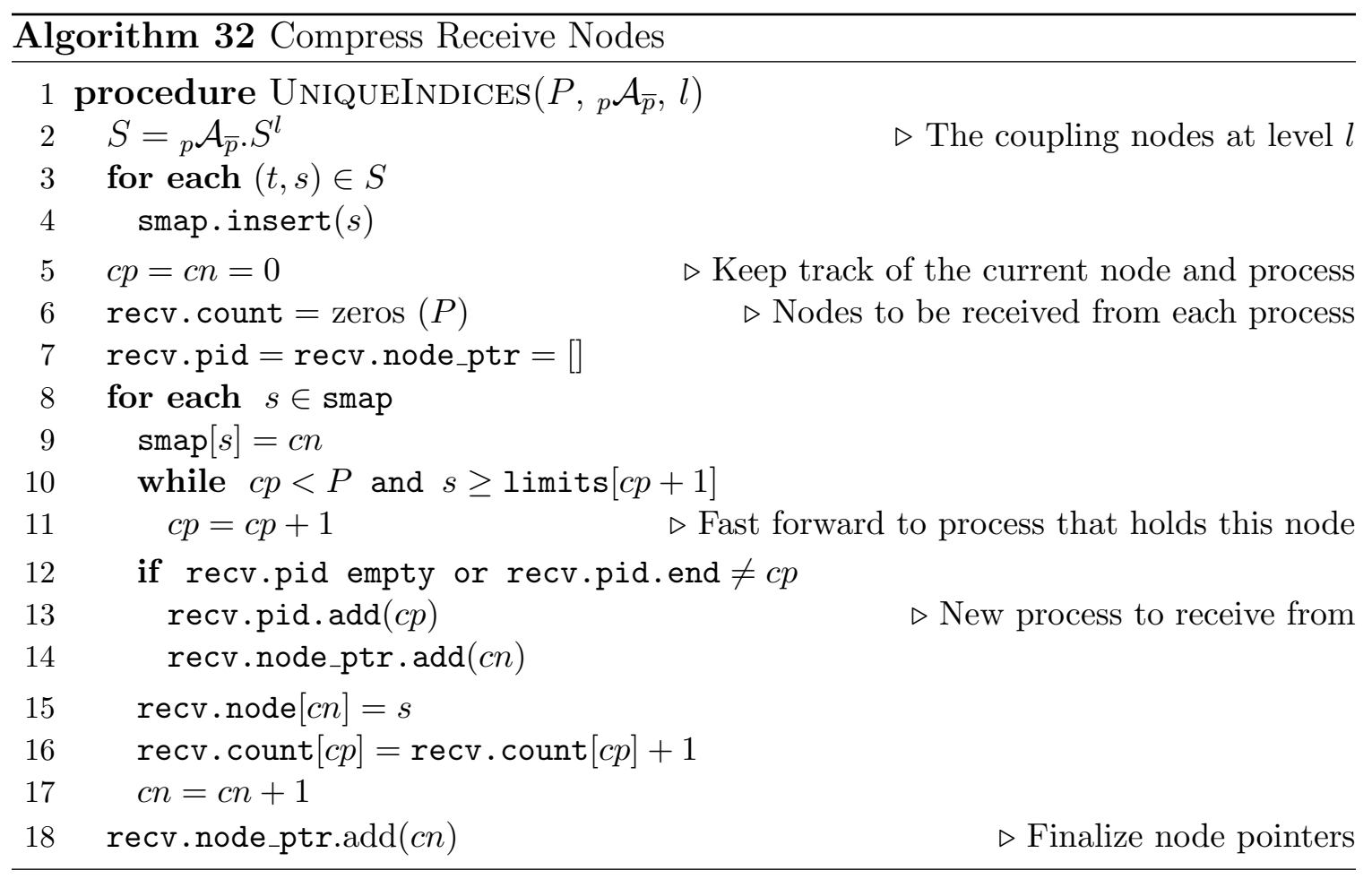

tell me which nodes GPU $p$ will need to complete its multiplication phase, so I must determine which GPUs the nodes belong to using the limits array. Since it is likely that on a level $l$ a GPU $p$ will not need to receive data from all $P$ GPUs, I determine a list of GPUs pid that need to send data to $p$ as well as a list of nodes that should be received node. The list of nodes will be ordered so that a separate list node_ptr will determine the limits within the array that each process in pid must send over; i.e., that a process pid $[i]$ must send over nodes in node from index node_ptr $[i]$ to node_ptr $[i+1]$. Algorithm 32 describes this process and Figure 8.3 shows an example of the resulting data for one level of an off-diagonal tree. The matrix tree $s$ node indices are then updated to use the new compressed receive indices.

The routine also determines how many nodes should be received from each process in the array count. Executing an all-to-all collective operation on that array allows all processes to exchange their receive counts into send counts, yielding the list of processors that must be sent to as well as the send node pointers. This exchange is shown in Algorithm 33. The list of receive nodes could be exchanged in the same way 


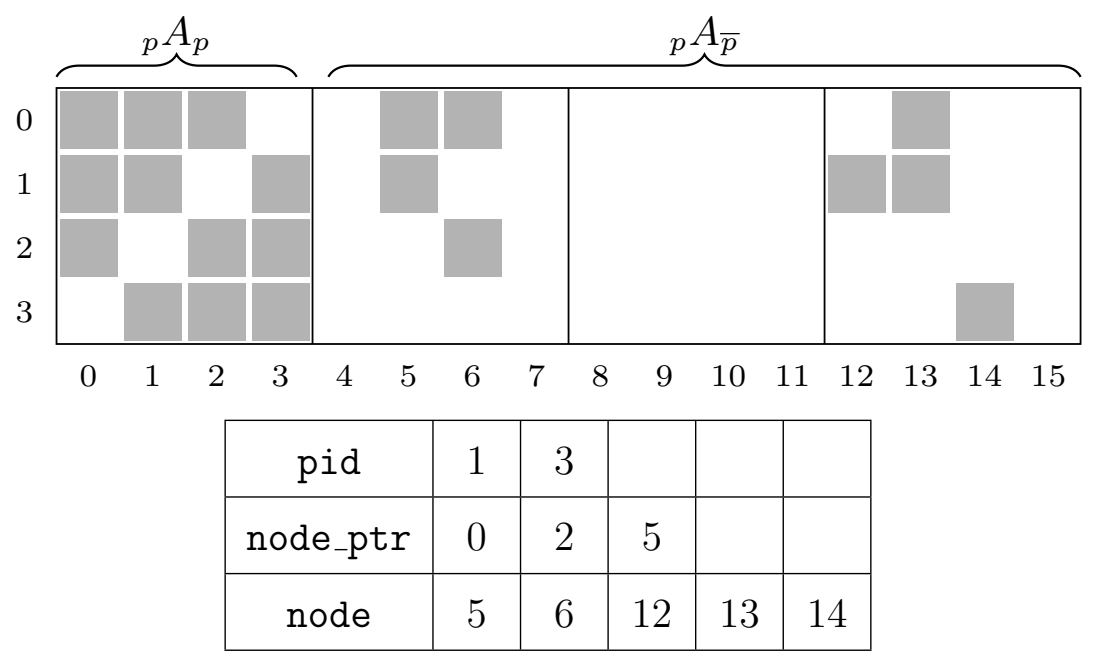

Figure 8.3: The compressed node data for the off-diagonal block ${ }_{p} \mathcal{A}_{\bar{p}}$ for a block row on process $p$ of a hierarchical matrix distributed to 4 GPUs. Process 2 has no corresponding basis tree data, so it is not included in the list pid

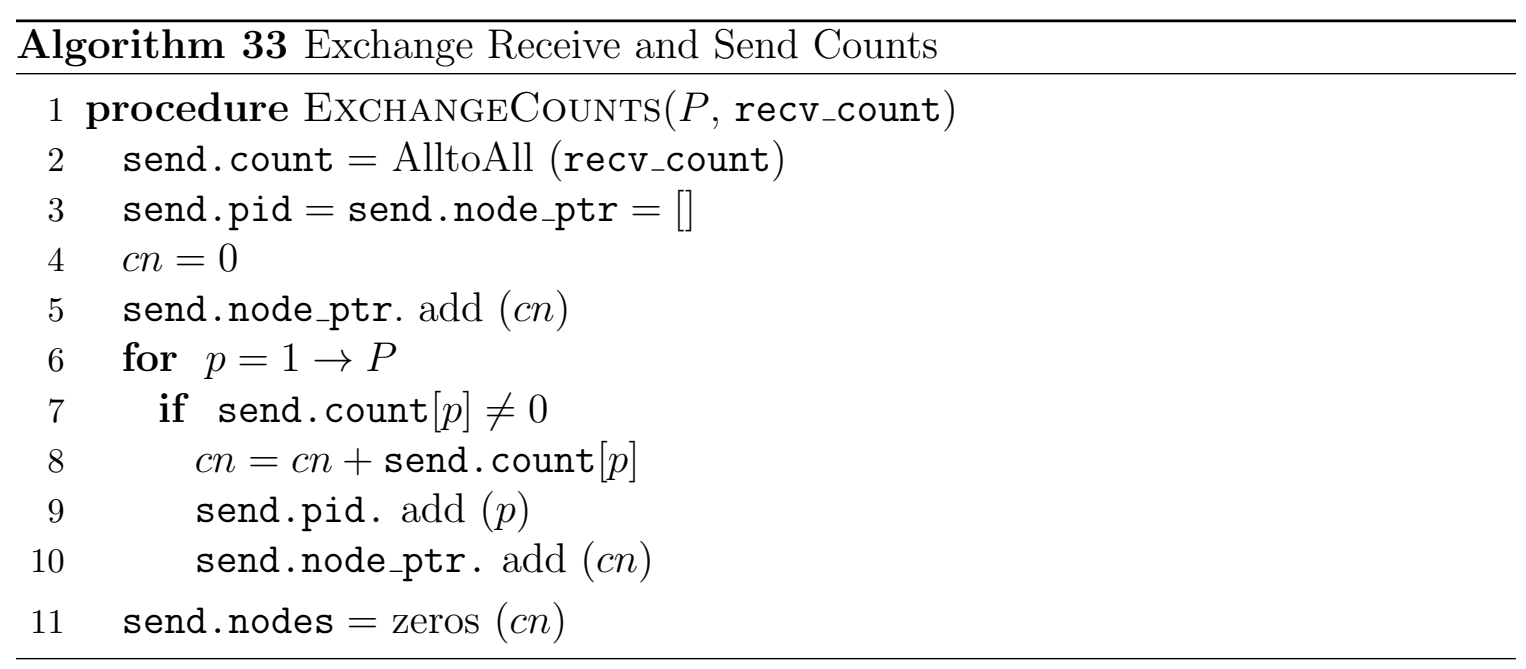

using the variable all to all collective, but since most lists will be empty anyway, it is more efficient to using simple nonblocking sends and receives instead as shown in Algorithm 34. This process is very similar to the way $\widehat{x}$ node data will be sent and received for the off-diagonal multiplication phase, which I discuss in the next section.

\subsubsection{Optimized Multiplication Phase}

Using the compressed off-diagonal data, the multiplication phase can be split into a diagonal phase and an off-diagonal phase. The diagonal phase can proceed with- 


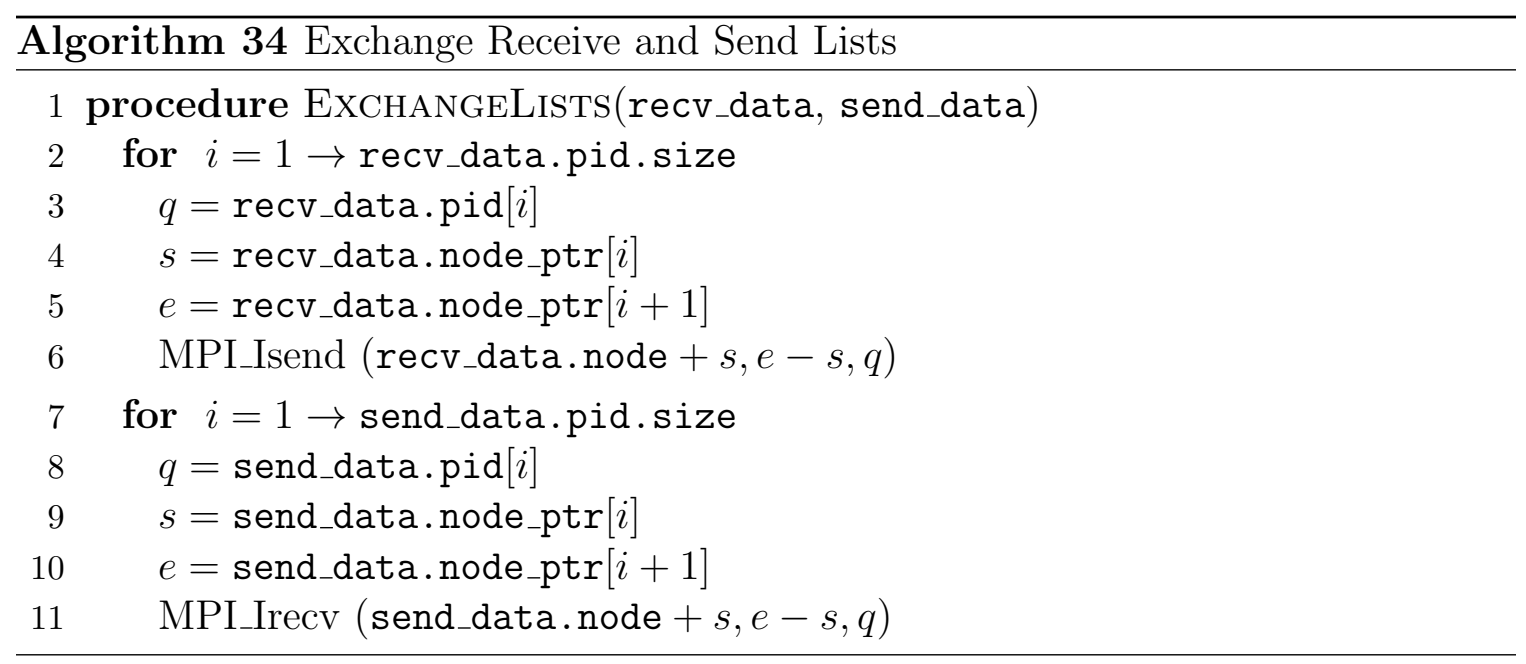

out any communication while the off-diagonal phase uses the node and process lists generated in Section 8.3.1 to exchange data. All nodes from a level $l$ of the local ${ }_{p} \widehat{x}$ branch listed in send_data.node are packed into a single buffer using the marshaling routine of Algorithm 35 and a single batched block copy kernel call into a send buffer $B_{s}^{l}$. Since the stride of the node data is determined by the fixed rank $k^{l}$ of the level, the marshaling is straightforward; however, in the case of variable ranks or leaf sizes, offsets into the buffer must be computed per node. This can be cached to save processing time, but must be updated if the ranks of the matrix change (for instance due to a low rank update or a compression operation). Since I use a fixed rank per level, the exchange of data into a receive buffer $B_{r}^{l}$ is simpler and carried out in Algorithm 36, where nonblocking sends and receives allow the communication to overlap with the diagonal phase. The receive buffer can be used directly with the off-diagonal level of the tree, since the column basis indices have already been updated to use the compressed node indices. Algorithm 37 shows the overall communication optimized multiplication phase. While line 7 of the algorithm does issue a Wait_All() call, since the exchange of data was issued before the work for the diagonal block, the process will likely not have to wait for long (or at all), depending on the structure of the tree, the capabilities of the communication network, and the compute capabilities of the GPU. 

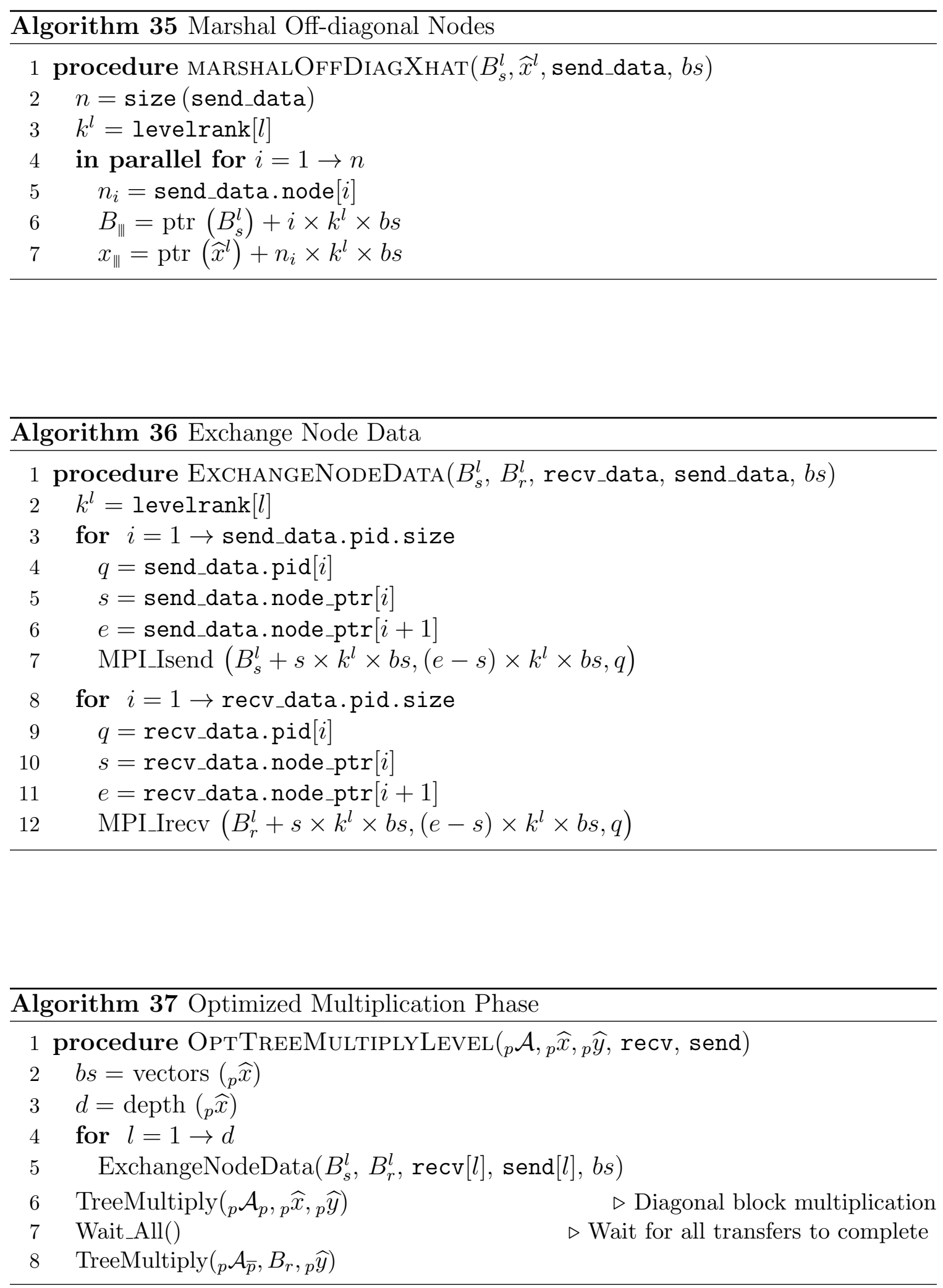


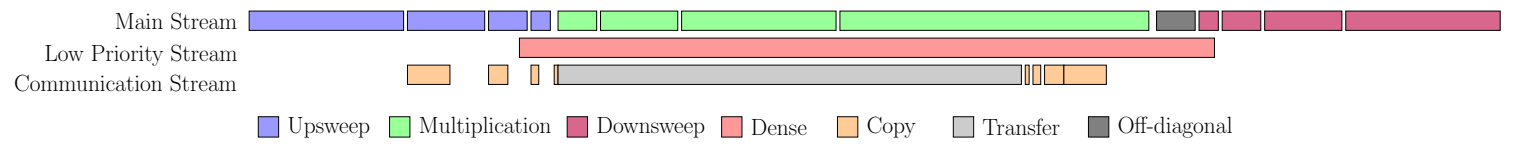

Figure 8.4: An example of the execution timeline for the distributed hgemv where the communication time is completely hidden by the processing of the diagonal blocks.

\subsection{Overlapping Communication with Computation}

While the communication volume has been reduced and a good portion of the communication can be hidden by overlapping it with the diagonal multiplication, there remain a few challenges that hold back performance. Assuming the lack of hardware support for more advanced memory transfer features such as GPUDirect Remote Direct Memory Access (RDMA), transferring the data from the GPU to the host so that the MPI library can be used adds some overhead to the execution as well as some synchronization points that impact GPU usage. To overcome this, I create a communication thread that queues up asynchronous copies on a separate stream to overlap the transfers with the processing of each level of the local branch upsweep. The transfers using MPI can then carried out on the same thread without interrupting or forcing synchronizations with the main execution stream, allowing communication and computation to overlap. When the diagonal block kernels have been queued up on the stream, the communication thread can then join the main thread. The multiplication of the root branch on only the master process, a branch that grows with $P$, can also be hidden (to an extent) by overlapping it with the main stream of work by scheduling it on a low priority stream. This will allow the work to be completed during phases of low GPU utilization, such as the smaller top levels of the basis and matrix tree. Finally, by adding the dense block multiplication phase to a low priority stream as I did for the single GPU hgemv, GPU utilization on all GPUs can be increased as well.

Figure 8.4 shows an example of the timeline for the overall distributed hgemv. 


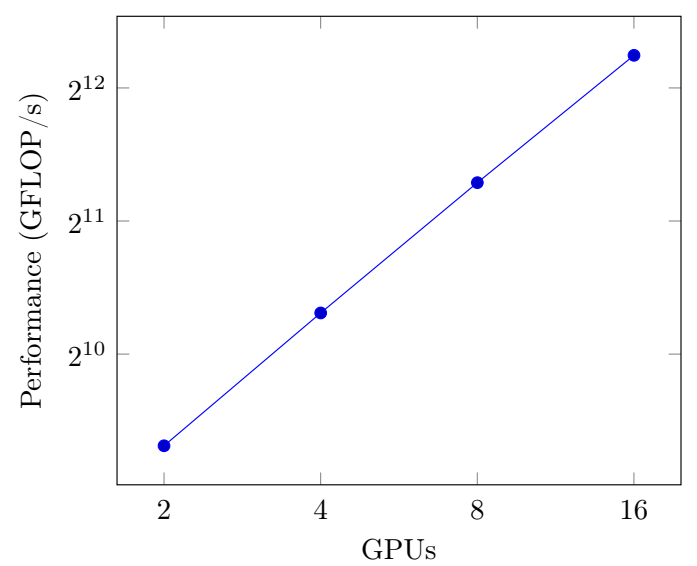

(a) Weak scaling as measured in GFLOPs.

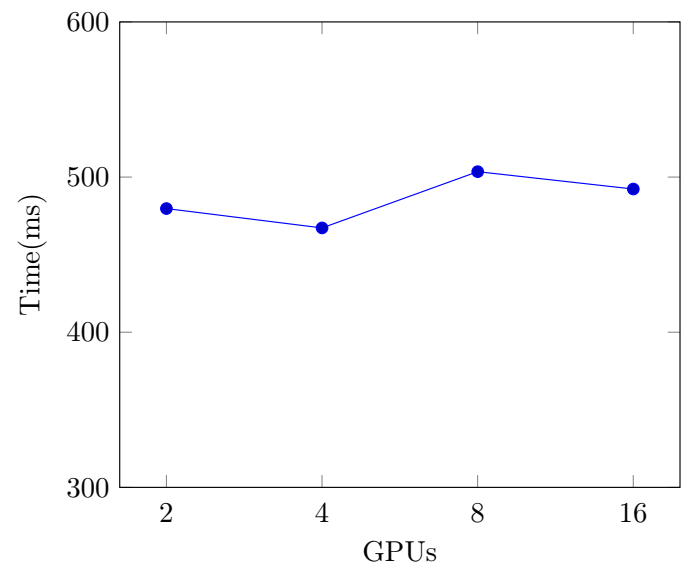

(b) Weak scaling as measured in time.

Figure 8.5: The weak scaling performance of the communication optimized distributed hgemv on up to 16 K80 GPUs with ${ }_{p} n=2^{19}$.

If the time process the diagonal work is greater than the communication time, then there will not be any gaps in the timeline corresponding to the communication thread joining the main thread. In that setting, the distributed algorithm scales perfectly as seen in the weak scaling performance of Figure 8.5, where up to 16 K80 GPUs each store and process ${ }_{p} n=2^{19}$ degrees of freedom.

\subsection{Scalability Results}

To test the performance and scalability of the implementation, I performed numerical experiments on the set of $2 \mathrm{D}$ and 3D covariance matrices described in Section 4.5. I used a number of vectors $n v$ ranging from 1 to 64 in these tests. Each test was performed on two machines with different network performance characteristics: Summit and a GPU cluster with a less performing communication infrastructure. Individual nodes on Summit have 6 NVIDIA V100 GPUs with 16GB of HBM2 memory each, but I only used 4 GPUs per node (2 per socket) in these runs (disabling the Volta Multiprocess Service), to allow a more direct comparison of performance with the results from the GPU cluster. Summit has a fast host-to-device bandwidth which can 
deliver up to $50 \mathrm{~GB} / \mathrm{s}$; the asynchronous transfers of the off-diagonal data to and from host buffers take advantage of a significant portion of it, $40 \mathrm{~GB} / \mathrm{s}$, as measured by nvprof. It also has a fat-tree topology network for internode communication that delivers $200 \mathrm{~Gb} / \mathrm{s}$ of bandwidth. In contrast, the GPU cluster, which also uses NVIDIA V100 GPUs but with 32GB of memory, has a host-to-device bandwidth of about 6-7 $\mathrm{GB} / \mathrm{s}$, and a network that can deliver only $32 \mathrm{~Gb} / \mathrm{s}$ between nodes. I do not exploit direct GPU-to-GPU communication on either system and utilize CPU buffers. To assess the efficiency attained by the algorithms, I measure the performance of the single GPU batched GEMM implementation from MAGMA, with batch elements of size $64 \times 64$. All computations are done in double-precision.

The 2D problem size was scaled using a grid size of ${ }_{p} N=2^{19}$ per GPU on Summit and ${ }_{p} N=2^{20}$ on the cluster because of the larger memories on the cluster GPUs. The hierarchical matrix representation of this covariance matrix uses $m=64$ as the finest block size and size of the dense leaves. A geometric admissibility condition $\eta\left\|C_{t}-C_{s}\right\| \geq\left(D_{t}+D_{s}\right) / 2$ is used in the dual tree traversal, where $C$ and $D$ refer to the center and diagonal size of a bounding box of the point sets $t$ and $s$. I use a value of $\eta=0.9$ and set a rank $k=64$ in the low rank blocks. The resulting sparsity constant of the matrix, which is a proxy for how finely refined the matrix is in its off-diagonal portions, is $C_{s p}=17$. At the largest size, the matrix has 23 levels, with the top 10 levels on a single master GPU, and the bottom 13 levels on separate 1024 GPUs for a matrix size of 536M.

The second matrix set comes from a 3D Gaussian process and is intended to show the effect of memory pressure - due to a finer refinement in the off-diagonal blockson scalability, particularly on a slower network communication infrastructure. The problem size is scaled using a grid size of ${ }_{p} N=2^{19}$ per GPU. I use a value of $\eta=1.1$ and set a rank $k=64$ in the low rank blocks. The resulting matrix tree has many more leaves than in the $2 \mathrm{D}$ case and a larger sparsity constant $C_{s p}=30$. 
Results from Summit are summarized in Figure 8.6, with the top and bottom rows showing the results from the $2 \mathrm{D}$ and $3 \mathrm{D}$ test sets, respectively. Every point in every plot was generated as the average of 10 runs after having discarded the fastest and slowest timings. There was generally no significant variability in the timings, with the highs and lows within $1-3 \%$ of the average. There is a slight jitter in the plots as the problems are scaled up, due to small changes in the structure of the matrix tree affecting the amount of actual computations performed, which do not grow exactly at the same rate as the problem size. The relative efficiency was computed as $\left(G_{P} / G_{P_{0}}\right) /\left(P / P_{0}\right)$, the ratio of the relative flops performed and the scaling factor relative to a base case of $P_{0}=2$ GPUs.

For the 2D tests, the scalability is near ideal up to 512 GPUs across all multiple vector sizes, $n v=1 \ldots 64$. For the single vector case, a bandwidth-limited operation, the throughput is about 150 Gflop/s per GPU. With $n v=64$, the additional arithmetic intensity pushes the computation into a compute-bound regime and achieves 2.6 Tflop/s per GPU, more than $95 \%$ of the sustained peak of the batched GEMM operation measured at 2.7 Tflop/s. Even with so much data volume being communicated (corresponding to various parts of 64 vectors), the communication is essentially hidden by the local computations. Only with 1024 GPUs, the plots show a slight degradation in performance and a deviation from ideal scalability, particularly for the $n v=64$ case, with performance dropping to 2.3 Tflop/s per GPU. The primary reason for this is that the root tree on the master GPU is now 10 levels deep and is of size $65 \mathrm{~K}$. The communication and computation on this sizeable top tree become a bottleneck. In order to scale up to this number of GPUs or larger, the top levels of the trees must be recursively split further as mentioned in Section 8.1, so that the top level work is small enough to be overlapped with other parts of the computation.

The results of the $3 \mathrm{D}$ problem test set display a similar behavior, but the communication overhead appears earlier. For the single vector case, results scale reasonably 
well with problem size. A relative efficiency of about $90 \%$ at 1024 GPUs is reached, with a performance of about $130 \mathrm{Gflop} / \mathrm{s}$ per GPU. As the number of vectors $n v$ increases however, the communication incurred for transferring all the relevant portions of the ${ }_{p} \widehat{x}$ vectors to the GPUs that need those data becomes substantial. This is in addition to the larger root tree difficulty mentioned above. The compute part of the operation grows sub-linearly with problem size because of the favorable increase in arithmetic intensity; however, the communication volume grows linearly and it can no longer be hidden by the now relatively faster compute phases. While a performance of 2.6 Tflop/s per GPU is reached for 2 GPUs, the performance at 1024 GPUs reaches only 1.1 Tflop/s, an efficiency less than 45\%. The aforementioned recursive distribution of the top levels as well as more direct GPU-to-GPU communication for improved bandwidth are needed to mitigate this.

Results from the 64-GPU cluster for both test sets are shown in Figure 8.7. While the trends are somewhat similar to those of Summit, the slower communication between GPUs shows its effect on scalability in a noticeable manner. For the single vector case in the $2 \mathrm{D}$ test set, where the overall volume of communication in the operation is small and the internal GPU bandwidth is the bottleneck, ideal scalability is achieved as seen in the constant time of the operation, and reaches $160 \mathrm{Gflop} / \mathrm{s}$ per GPU. As above, for the 64-vector case, the compute part of the operation grows sublinearly because of the increased arithmetic intensity but the communication volume grows linearly, and on a slower communication network rapidly reduces scalability. On 4 GPUs, the performance of the 64 vector case reaches 2.4 Tflop/s per GPU, essentially the peak value reached by a batched GEMM on one of these GPUs, while on 64 GPUs drops to 2.0 Tflop/s per GPU - a relative efficiency of about $80 \%$ - as the slow communication can no longer be hidden by other parts of the computation. In the 3D test set, with its larger communication demands due the bushier structure of the matrix tree, the scalability is adversely affected by the slow communication. 
In the 64-vector case the performance reached drops from $1.4 \mathrm{Tflop} / \mathrm{s}$ per GPU with 4 GPUs to only 0.6 Tflop/s, a relative efficiency dropping down to below $45 \%$. The relative efficiency in the single vector case however is not affected much, as the overall communication volume is low, and remains at about $95 \%$. 

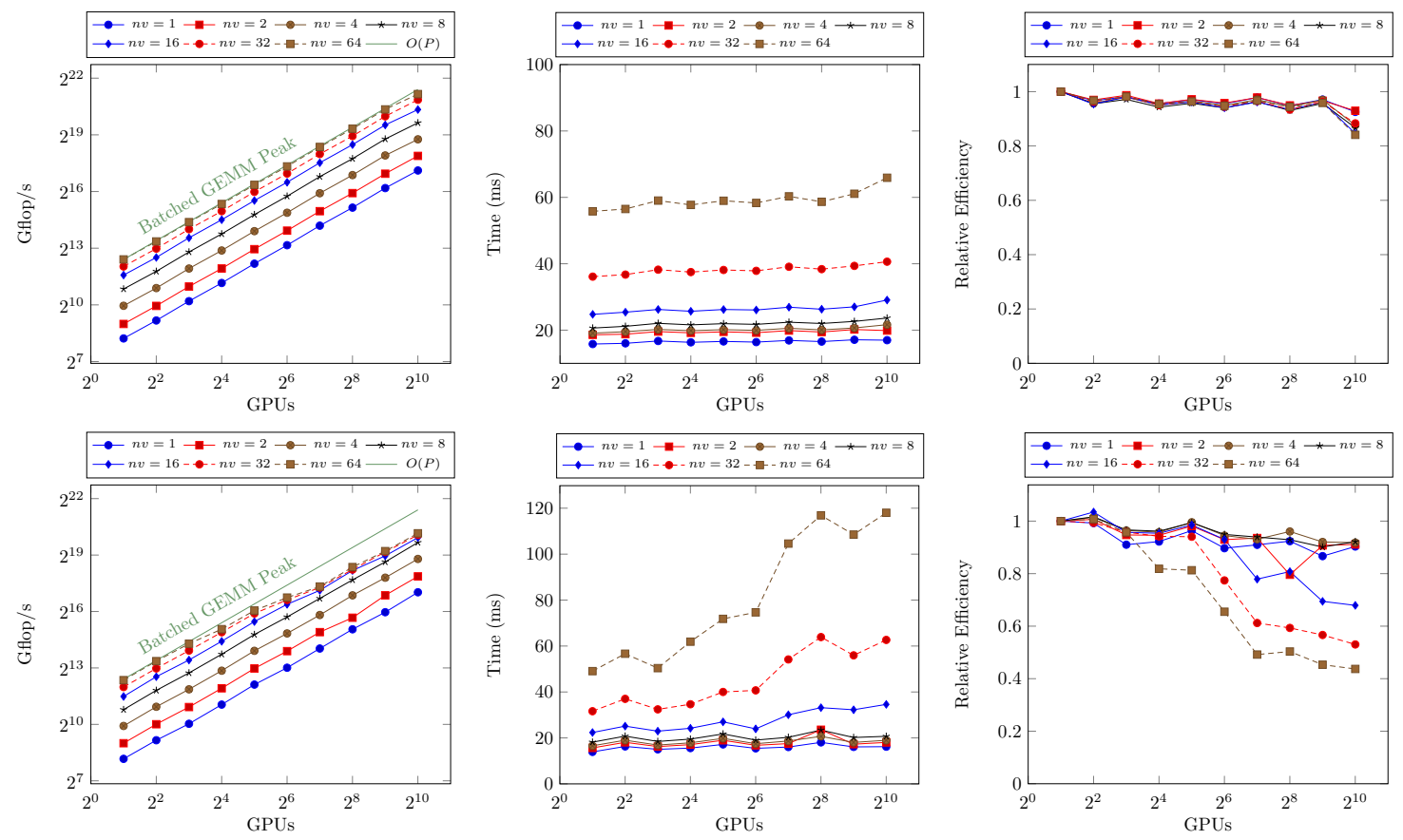

Figure 8.6: Scalability and efficiency of HGEMV on SUMMIT, on two sets of matrices.
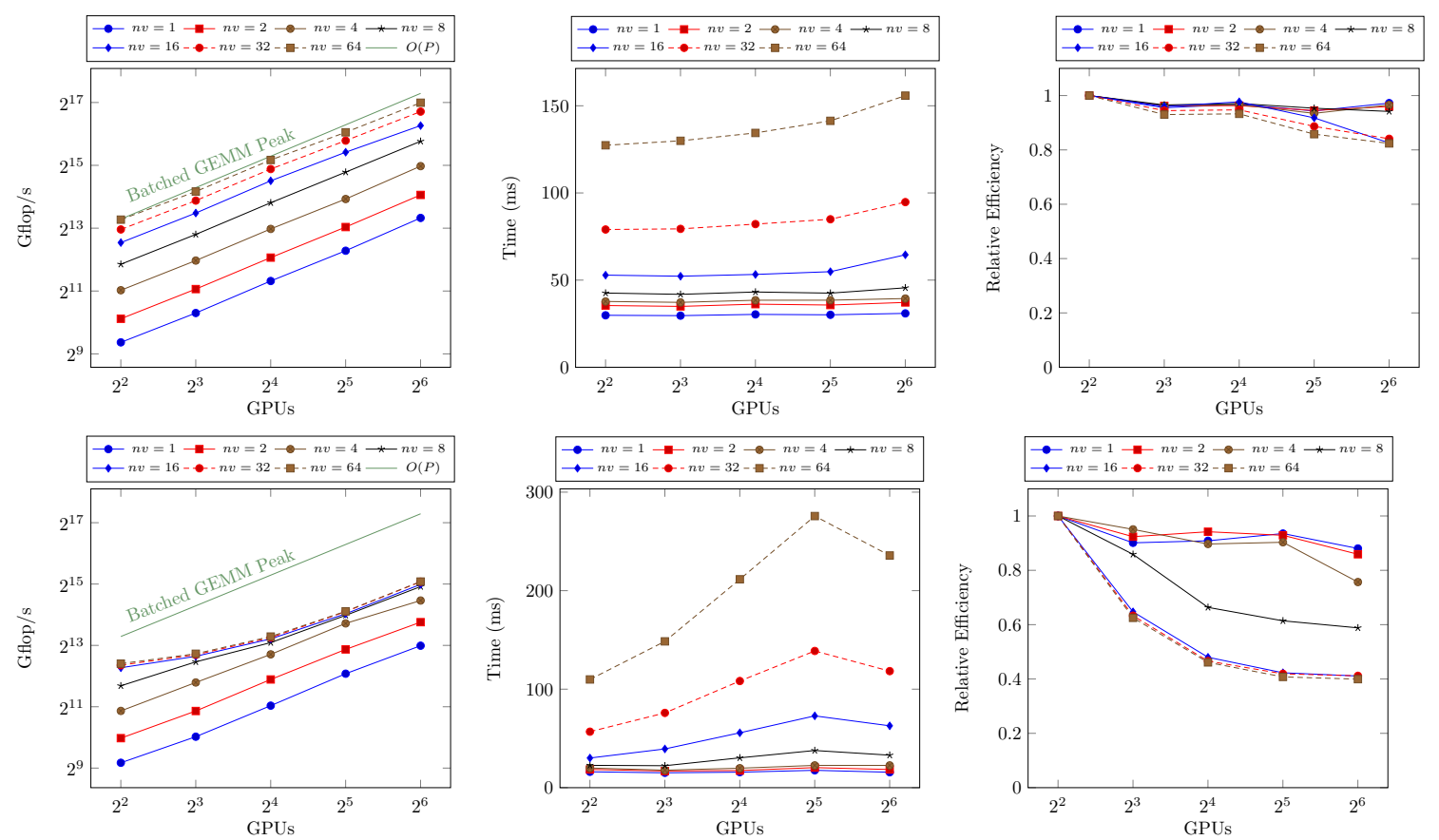

Figure 8.7: Scalability and efficiency of HGEMV on a 64-GPU cluster, on two sets of matrices. 


\section{Chapter 9}

\section{Application: Hessians Arising in Inverse Problems Governed by PDEs}

In this chapter, I show the efficiency of hierarchical matrix representations in representing the Hessian operators of PDE-constrained problems, and consider these tunable-accuracy approximations to address the shortcomings of globally low rank representations in the increasingly data-informed regime, as well as to provide loglinear time complexity algorithms for constructing robust approximations of the inverse of these operators.

The Hessian operator plays a central role in inverse problems involving optimization of systems governed by partial differential equations (PDEs), also known as PDE-constrained optimization. The goal of an inverse problem is to infer model parameters, given observational data, a forward model or state equation (here in the form of PDEs) mapping parameters to observables, and any prior information on the parameters. Often the parameters represent infinite-dimensional fields, such as heterogeneous coefficients (including material properties), distributed sources, initial or boundary conditions, or geometry. I focus here on the infinite dimensional setting, leading to large scale inverse problems after discretization.

The Hessian operator plays a critical role in inverse problems. For deterministic inverse problems, finding the parameters that best fit the data is typically formulated as a regularized nonlinear least squares optimization problem. Its objective function(al) consists of two terms: the data misfit, that is, the squared $\ell^{2}$-norm of the difference between the output observables predicted by the PDE for given model 
parameters and the observed data; and a regularization term that ensures stability by penalizing unobserved features of the parameters, such as rough components. The Hessian is given by the second variation, with respect to model parameters, of this regularized data misfit. Minimizing the objective by Newton's method requires solution of a sequence of linear systems with the Hessian as its system matrix. Due to its affine invariance and resulting mesh-independent behavior (e.g., [91]), Newton's method is the gold standard for inverse problem solution, with demonstrated convergence independent of parameter dimension for large-scale, complex inverse problems (e.g., [92, 93]). Efficient solution of the linear systems arising at each Newton iteration requires an effective preconditioner that is inexpensive to form and "invert." The challenge is that the discretized Hessian of the data misfit functional is formally a dense operator that cannot be formed explicitly, since each column requires the solution of a linearized state PDE. Instead, we can exploit the fact that for ill-posed inverse problems, the data misfit Hessian operator is compact (its eigenvalues accumulate at zero), and when the regularization operator takes the typical form of an elliptic differential operator, we can precondition by the inverse of the regularization to yield a preconditioned Hessian in the form of a compact perturbation of the identity. A conjugate gradient linear solver will converge in a number of iterations that depends on the number of distinct eigenvalues, which for regularization preconditioning is small when the compact part has eigenvalues that decay rapidly, so that it has small effective global rank $r$. Since at each iteration, applying the Hessian to a vector requires the solution of a pair of linearized state/adjoint PDEs, $O(r)$ such pairs must be solved at each Newton iteration. When $r$ is small this strategy is effective, however the central challenge is that $r$ grows as the data become more informative (a desirable situation for inverse problems), and regularization preconditioning becomes prohibitive.

While $r$ generally does not grow with increasing parameter dimension, it does 
grow as the data become more informative about the parameters. To illustrate this fact, consider a linear Bayesian inverse problem with additive Gaussian noise. The Kullback-Leibler divergence (or relative entropy) from posterior to prior measurewhich quantifies the information gained from the data - can be shown to be equal to $\Sigma_{i} \log \left(\lambda_{i}+1\right)$, where $\lambda_{i}$ are the eigenvalues of the prior-preconditioned data misfit Hessian [94]. This sum can be truncated when $\lambda_{i} \ll 1$, so the dominant eigenvaluesand hence effective rank of this operator-are directly related to the information content of the data. The information gained from the data (and hence $r$ ) generally increases as the number of sources (or experiments) increases and the number of observations (or sensors or receivers) increases. The data also become more informative as the state PDEs become less dissipative (for example as flow or transport equations become more advection-dominated), or as they resolve finer scales (such as in wave propagation with increasing frequency). Finally, as the noise in the data decreases, the strength of the data misfit Hessian increases relative to the prior/regularization, again increasing the effective rank and hence informativeness of the data. In all such cases, both regularization preconditioning as well as globally low rank-based data misfit Hessian approximation become prohibitive, and there remains a critical need for more efficient Hessian approximation.

Hierarchical matrix representations present an elegant solution to this need. In the rest of this chapter I present numerical experiments to assess the effectiveness of the hierarchical matrix Hessian representations on inverse problems governed by diffusion and transport PDEs. The experiments demonstrate the superiority of hierarchical matrix approximations over a globally low rank approximation as the data become more informative, with speedups ranging from two to over an order of magnitude. 


\subsection{Why do Hessians Admit Hierarchical Low Rank Repre- sentations?}

The Hessians that arise in PDE-governed inverse problems (whether deterministic or Bayesian) may be approximated to specified accuracy by highly compressible matrices, a property they inherit from the underlying PDE operators [95], often reinforced by sparse observation operators. As argued above, the global rank of these Hessians increases as the data become more informative, such that a low rank approximation may no longer be tenable.

However, as described below, these Hessians typically have blocks that can be well approximated by low rank representations with bounded rank. These blocks are at different levels of granularity because of their different sizes, making hierarchical matrix representations an ideal vehicle for storing and computing with Hessians.

For illustration, I consider inverse problems governed by PDEs with distributed parameter fields to be inferred from data. After discretization over a $d$-dimensional domain $\Omega$, an optimization problem of the following form is obtained:

$$
\underset{m}{\operatorname{minimize}} J(m):=F(u(m))+\alpha R(m)
$$

where the state variables $u(m) \in \mathbb{R}^{N}$ depend on the model parameters $m \in \mathbb{R}^{n}$ via solution of the discretized PDEs

$$
g(m, u):=K(m) u-f=0
$$

with coefficient matrix $K \in \mathbb{R}^{N \times N}$ and source $f \in \mathbb{R}^{N}$. For simplicity, the discussion is confined to the case where the forward problem $g(m, u)=0$ represents elliptic PDEs that have arbitrary dependence on the parameter but are linear in the state. However, the methodology is more broadly applicable to parabolic and hyperbolic 
forward problems, as demonstrated in the applications in Section 9.2, and to nonlinear forward problems. Multiple sources (or multiple experiments) may be used to generate data, in which case 9.2 is indexed by source $s$, generating the state $u^{s}$.

The data misfit term $F(u(m))$ measures the difference between recorded observations and the response of the model at receivers placed on the boundary or the interior of $\Omega$. It is often taken to be the sum of squares, over all sources and receivers, of $d_{r}^{s}$, the difference between the observed state at receiver $r$ due to source $s$, and the corresponding model response $u_{r}^{s}$,

$$
F:=\frac{1}{2} \sum_{s} \sum_{r}\left\|u_{r}^{s}-d_{r}^{s}\right\|^{2}
$$

although other measures of misfit may be sometimes preferable [96]. $R(m)$ is a regularization term expressing prior information on the parameter field (such as piecewise smoothness) and $\alpha$ is a scalar regularization parameter that attempts to annihilate components of $m$ that are uninferable from the data while preserving those that can be inferred. It is generally a local operator whose discretization is sparse and does not pose computational difficulties even at large scale. Thus in this section I focus on the treatment of the problematic $F$ term, whose evaluation requires solution of the forward PDEs for a given $m$ for each source $s$.

Define $G \in \mathbb{R}^{N \times n}$ to be the partial derivative of the forward PDE residual with respect to the parameter field, i.e.,

$$
G:=\partial_{m} g=\partial_{m} K \times_{2} u
$$

where $\partial_{m} K$ is a third order tensor of size $N \times N \times n$ and $\times_{2}$ is the 2-mode tensor vector product operation in the notation of [97]. Since $K$ is a discretization of a local PDE operator, it is representable as a sparse matrix. The parameter field is typically discretized on the same mesh as the state (perhaps at lower order), and thus the local 
operator $G$ is also sparse.

Unlike the local sparse matrices $K$ and $G$, the inverse operator $K^{-1}$ is a discretized, non-local solution operator, and is in general formally dense. It is however datasparse, since many of its off-diagonal blocks can be represented to high accuracy by low rank approximations with bounded rank $k$, and these blocks occur at different levels of granularity [95, 5]. For a block $t s$ of $K^{-1}$, with rows corresponding to nodes $t$ and columns corresponding to nodes $s$ of a finite element mesh, the compressibility condition states that if the bounding boxes of these node clusters are sufficiently far away from each other, then the local rank of the ts block is essentially independent of the block size for Poisson-like operators.

Using the nested basis $\mathcal{H}^{2}$ representation [82], the total amount of storage needed to represent $K^{-1}$ to a given accuracy $\epsilon$ is only $O(N k)$, where the local block rank $k$ grows as $|\log \epsilon|^{d+1}$ for PDEs in $d$ spatial dimensions. Note that the local ranks of the blocks are independent of the global rank. Even for matrices that are of full rank $N$, the local ranks grow slowly, with only a logarithmic dependence on target approximation accuracy, as well as with a logarithmic dependence on high contrast coefficients [98]. High-frequency problems can be tackled effectively by using directional compression techniques, with asymptotic storage $O\left(N k^{2} \log N\right)[99$.

The gradient of the data misfit term of the objective function may be written in terms of an adjoint variable $p \in \mathbb{R}^{N}$, defined via the adjoint equations [100]

$$
l(m, p):=K^{T}(m) p+\partial_{u} F=0
$$

The gradient of $F$ is then given by

$$
\nabla_{m} F:=\left(\partial_{m} K \times_{2} u\right)^{T} p=-G^{T} K^{-T} \partial_{u} F
$$

I denote by $L \in \mathbb{R}^{N \times n}$ the partial derivative of the residual of the adjoint equations 
(9.3) with respect to $m$,

$$
L:=\partial_{m} l=\partial_{m} K^{T} \times_{2} p
$$

which is a local operator and has a sparsity pattern similar to that of $G$. Taking the derivative of 9.4 with respect to $m$, I obtain an expression for the Hessian matrix in terms of the state and adjoint variables $u$ and $p$, and the local operators $G$ and $L$,

$$
\nabla_{m m}^{2} F=G^{T} K^{-T} \partial_{u u}^{2} F K^{-1} G-G^{T} K^{-T} L-L^{T} K^{-1} G+\left(\partial_{m m}^{2} K \times_{2} u\right) \times_{1} p
$$

The first term of the latter expression represents the positive semi-definite GaussNewton part of the Hessian, involving the application of the local operator $G$ and its adjoint from the left and right to the triple product $K^{-T}\left(\partial_{u u}^{2} F\right) K^{-1}$. The next two terms involve the application of the sparse Jacobians of the state and adjoint equations, from the left and from the right, to $K^{-1}$ and its adjoint $K^{-T}$. The last term involves a sparse, fourth-order tensor multiplied in 1-mode and 2-mode by the adjoint and state variables.

Equation (9.5) shows why it is prohibitive to form the Hessian operator explicitly: forming $K^{-1} G$ (and similar products) requires a number of forward PDE solves equal to the number of parameters multiplied by the number of sources. On the other hand, (9.5) also shows that products of the Hessian with arbitrary vectors can be carried out at the cost of one forward solve (with $K$ ) and one adjoint solve (with $K^{T}$ ). Note also that (unless the forward problem is linear in $m$ ), in general $u, p, K, G$, and $L$ all depend on $m$, so the Hessian varies over parameter space. However the argument below is not predicated on where in parameter space the Hessian is evaluated.

The form (9.5), shows why the Hessian of 9.1) exhibits a hierarchical low rank structure. Consider first the Gauss-Newton term. The observation operator $\partial_{u u}^{2} F$ is positive semi-definite, and in particular is block diagonal for the standard weighted least squares objective (where the blocks correspond to the support of the receiver 
operators). When the number of observations in $F$ is small, it could have relatively small global rank as well. A general result concerning the structure of the product

of hierarchical matrices [67] shows that the triple product $K^{-T} \partial_{u u}^{2} F K^{-1}$, even when $\partial_{u u}^{2} F$ is full rank, may be represented as a hierarchical matrix, although the local ranks may grow as a result. The right and left multiplications of the latter triple product by the sparse matrix $G$ and its transpose have the effect of reducing the local ranks. This is due to the fact that a block in the product contains contributions from a bounded number of block products, where most of the operands involved are zero and contribute no data to the target block. The local ranks of the Gauss-Newton Hessian may therefore end up being smaller that the local ranks of the solution operator. The second and third terms of the full Hessian expression given in (9.5) also have the form of a hierarchical matrix multiplied on both sides by sparse matrices, and therefore result in hierarchical matrices with small local ranks. Finally, the last term of the Hessian involves the sparse fourth order tensor. The sum of all four terms may of course increase the local ranks slightly, but the resulting Hessian will still retain a hierarchical structure.

\subsection{Illustrative Applications}

In this section, I discuss the effectiveness of the hierarchical representation of Hessians for several inverse problems, governed by a time-dependent diffusion equation in one spatial dimension (Section 9.2.1), and in two space dimensions by a steadystate advection-diffusion equation (Section 9.2.2). I focus on the approximations of the Hessian evaluated at the parameter field found as the solution of the optimization problem. I study the cost in terms of the number of Hessian applications (in PDE-constrained optimization this is usually the dominating cost) required for the construction of the hierarchical matrix approximations, and compare it with the cost of global low rank approaches. I also study the influence of the number of observations 
and properties of the governing PDEs on the compressibility of Hessian matrices.

\subsubsection{Density Inversion in 1D Time-dependent Diffusion Equa- tion}

I consider a one-dimensional domain where I seek to invert for a spatially-varying porosity coefficient $\rho(x)$ in the governing diffusion equation $\rho(x) \partial_{t} u-\partial_{x}^{2} u=w(x, t)$. I use 3 sources and 8 receivers placed as shown in Figure 9.1. The point sources produce a Ricker wavelet [101] time history input $W$ and the signals are recorded at the receivers until they have effectively dissipated at a final time $T>0$.
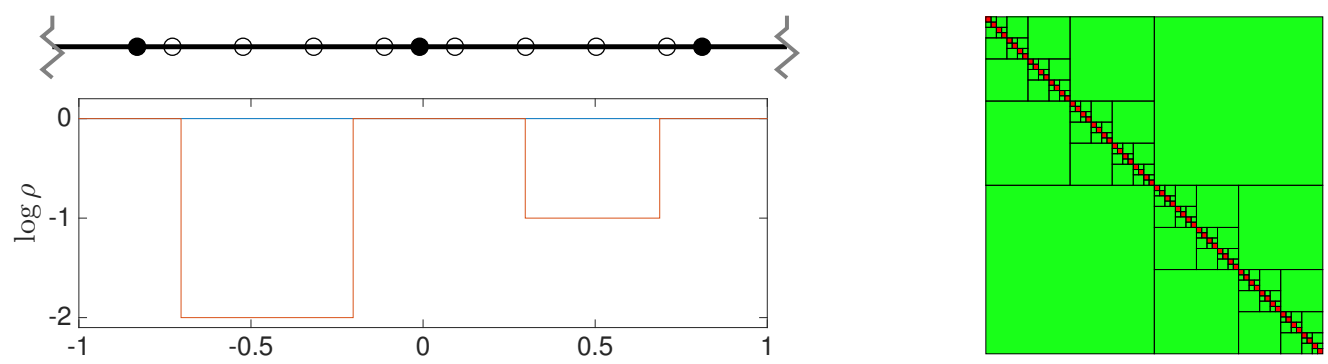

Figure 9.1: (left) Sources $(\bullet)$, receivers (o), and target profile; (right) Hessian structure.

The medium to be recovered is in the interval $-1 \leq x \leq 1$ and has sharp discontinuities (see Figure 9.1), prompting the use of a (smoothed) total-variation regularization such that the optimization formulation is:

$$
\underset{\rho:[-1,1] \rightarrow \mathbb{R}}{\operatorname{minimize}} J(\rho):=\frac{1}{2} \sum_{s} \sum_{r} \int_{0}^{T}\left(u_{s}\left(x_{r}, t\right)-d_{r}(t)\right)^{2} d t+\alpha \int_{-1}^{1} \sqrt{\left|d_{x} \rho\right|^{2}+\beta} d x
$$

where for all sources $s, u_{s}(x, t)$ is the solution to

$$
\begin{aligned}
\rho(x) \partial_{t} u_{s}-\partial_{x}^{2} u_{s} & =\delta\left(x-x_{s}\right) W\left(t-t_{0}\right), & & |x|<\infty, 0 \leq t \leq T, \\
u_{s}( \pm \infty, t) & =0, & & \text { (Dirichlet BC) } \\
u_{s}(x, 0) & =0 . & & \text { (IC) }
\end{aligned}
$$


Here, the first term of the objective is the least squares time misfit summed over all sources and receivers. The second term is a total-variation regularization functional that allows sharp discontinuities to be recovered, where $\alpha>0$ is the regularization weight and a small $\beta>0$ ensures that the objective is differentiable.

For the computations below, $\rho(x)$ is discretized in $[-1,1]$ using $n$ linear elements. A finite element discretization of the governing equation with the same mesh is used in $[-1,1]$ and extended outside with constant $\rho=1$ to apply the homogeneous Dirichlet boundary conditions at a sufficiently far distance. An implicit second-oder time integration scheme is used for solving the semi-discrete equations.

The computation of the gradient may be done at the cost of two PDE solutions, a forward and an adjoint. The continuous form of the gradient of $J$, its functional Fréchet derivative, is

$$
\frac{\delta J}{\delta \rho}(x)=\sum_{s} \int_{0}^{T} \partial_{t} u_{s}(x, t) p_{s}(x, t) d t
$$

where, for each source $s, u_{s}$ is the solution of the state equation (9.7), and $p_{s}$ the solution of the corresponding adjoing equation that must be solved backwards-in time:

$$
\begin{gathered}
\text { (adjoint) }-\rho(x) \partial_{t} p_{s}-\partial_{x}^{2} p_{s}=-\sum_{r} \delta\left(x-x_{r}\right)\left(u_{s}\left(x_{r}, t\right)-d_{r}(t)\right) \\
q( \pm \infty, t)=0 ; \quad q(x, T)=0 .
\end{gathered}
$$

To compute a hierarchical representation of the Hessian, I need the ability to compute products of the Hessian of the misfit term with vectors. This may be done at the cost of solving, for each source $s$, two additional forward-like and adjoint-like PDEs. The continuous form of the Hessian-vector product, i.e., the product of the Fréchet Hessian with a model perturbation $\nu(x)$ is: 


$$
\frac{\delta^{2} J}{\delta \rho^{2}} \nu=\sum_{s}\left(\int_{0}^{T} \partial_{t} u(x, t) q(x, t) d t+\int_{0}^{T} \partial_{t} v(x, t) p(x, t) d t\right)+\frac{\delta^{2} R}{\delta \rho^{2}} \nu
$$

where the last term denotes the Hessian of the TV regularization $R$, and $v_{s}$ and $q_{s}$ are the solutions of the following incremental or second order forward and adjoint equations:

( $2^{\text {nd }}$ order forward)

$$
\begin{gathered}
\rho(x) \partial_{t} v_{s}-\partial_{x}^{2} v_{s}=-\nu(x) \partial_{t} u_{s}(x, t) \\
v_{s}( \pm \infty, t)=0 ; \quad v_{s}(x, 0)=0 \\
-\rho(x) \partial_{t} q_{s}-\partial_{x}^{2} q_{s}=-\sum_{r} \delta\left(x-x_{r}\right) v_{s}\left(x_{r}, t\right)-\nu(x) \partial_{t} p_{s}(x, t), \\
q_{s}( \pm \infty, t)=0 ; \quad q_{s}(x, T)=0 .
\end{gathered}
$$

The structure of the $\mathcal{H}$-representation of the Hessian is depicted in the right panel of Figure 9.1. I used a weak admissibility partitioning of the hierarchical matrix as appropriate for a one-dimensional spatial domain. There are $2^{l}$ symmetric blocks pairs at level $l$, each of size $n / 2^{l}$. For this example of size $n=2048$, I use 6 levels in the hierarchy, stopping the matrix refinement at blocks of size $m=32$. I construct the Hessian to a relative accuracy of about $10^{-6}$ in the 2-norm. The local ranks of the resulting matrix are shown in the left panel of Figure 9.2. The ranks plotted are the maximum local ranks for all off-diagonal blocks for every level. Even though the Hessian is full rank because of the regularization term, I note that its local ranks are small relative compared to its size even when a relatively tight tolerance of $10^{-6}$ is used in the construction.

Given this explicit representation of the Hessian, I can invert it to produce an explicit inverse. The convergence behavior of Newton Schulz starting from a scaled identity initial iterate to a relative accuracy of $\epsilon=10^{-6}$ is shown in Figure 9.2. The number of samples needed to construct the hierarchical approximation of the iterates 

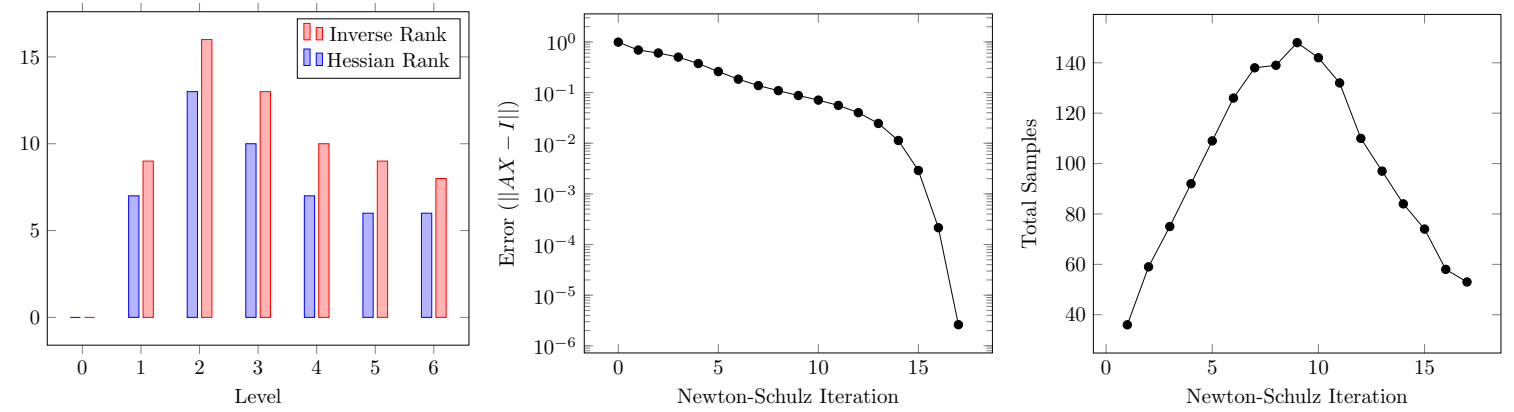

Figure 9.2: (left) Local ranks of Hessian and its inverse. (middle) Convergence history of NS. (right) Number of samples needed to construct the matrix over the iteratons.

is shown as well. In this simple 1D setting, I used a static threshold and a constant accuracy in the iterates. The increase in the required samples, and the corresponding local ranks, of the intermediate iterates is noteworthy. The left panel of Figure 9.2 shows the local ranks of the inverse along with the Hessian local ranks for comparison. There is little change in the local ranks in the inversion operation.

\subsubsection{Source Inversion in Stationary Advection-diffusion}

I consider a linear source inversion problem, i.e., I infer the right hand side source $m(x)$ in an advection-diffusion-reaction equation from point observations of its solution on a domain $\Omega \subset \mathbb{R}^{2}$. This amounts to the following optimization problem:

$$
\underset{m: \Omega \rightarrow \mathbb{R}}{\operatorname{minimize}} J(m):=\frac{1}{2 \sigma^{2}} \sum_{r}\left(u\left(x_{r}\right)-d_{r}\right)^{2}+R(m),
$$

where $u$ is the solution of

$$
\begin{aligned}
-\operatorname{div}(\kappa \nabla u)+\mathbf{v} \cdot \nabla u+c u & =m & & \text { in } \Omega, \\
u & =0 & & \text { on } \Gamma_{D}, \\
\kappa \frac{\partial u}{\partial n} & =0 & & \text { on } \Gamma_{N} .
\end{aligned}
$$


Here, the measurements $d_{r}$ are assumed to contain additive independent and identically distributed Gaussian noise with variance $\sigma$, and $R(m)$ is a regularization term. The coefficients $\kappa, \mathbf{v}$ and $c$ represent the diffusivity, the advective velocity and the reaction constant, respectively, and $\Gamma_{D}, \Gamma_{N} \subset \partial \Omega$ is a splitting of the boundary $\partial \Omega$, where I impose Dirichlet or Neumann boundary conditions, respectively.
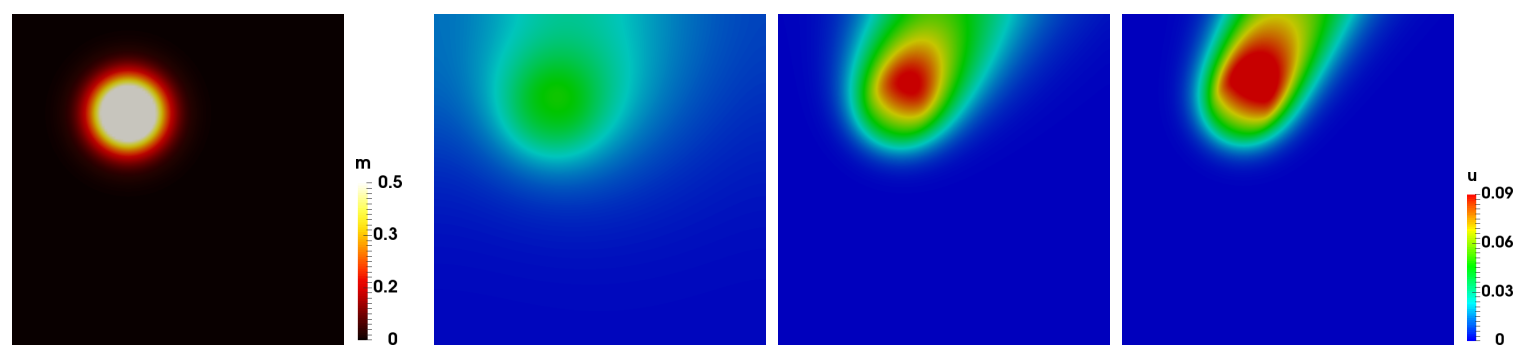

Figure 9.3: Dependence of the solution for different diffusion parameters $\kappa$ : Shown on the left is the source $m$ entering on the right hand side of (9.11). The right three plots show the solutions of the state equation for $\kappa=10^{-1}, 10^{-2}, 10^{-3}$ (from left to right). Depending on $\kappa$, the problem is diffusion- or advection-dominated.

For this study of the Hessian, I use $\Omega=[0,1]^{2}$, the physical parameters $c=0.5$ and $\mathbf{v}=\left(x_{1}, x_{2}\right)^{T}$, and a noise level of $1 \%$. To study the influence of the diffusion and the number of observations, I use different values of $\kappa \in\left\{10^{-3}, 10^{-2}, 10^{-1}\right\}$ and different numbers of observation points, $N_{o b s} \in\{250, \ldots, 16000\}$.

I discretize $\Omega$ using triangles based on a uniform grid of size $128 \times 128$, and use linear continuous finite elements to discretize the parameter $m$. The presence of strong advection in the problem mandates the use of stabilization for the state and adjoint variables. A first-order Symmetric Interior Penalty Galerkin (SIPG) scheme [102, 103] with upwinding [104] is used for both the forward and adjoint problems. The implementation is based on FEniCS [105] and hIPPYlib, an open-source library providing scalable adjoint-based algorithms for PDE-based inverse problems [106]. The true source and the state solutions corresponding to the different values of the diffusion coefficient are shown in Figure 9.3 .

In this study of the compressibility of the Hessian and the associated compu- 

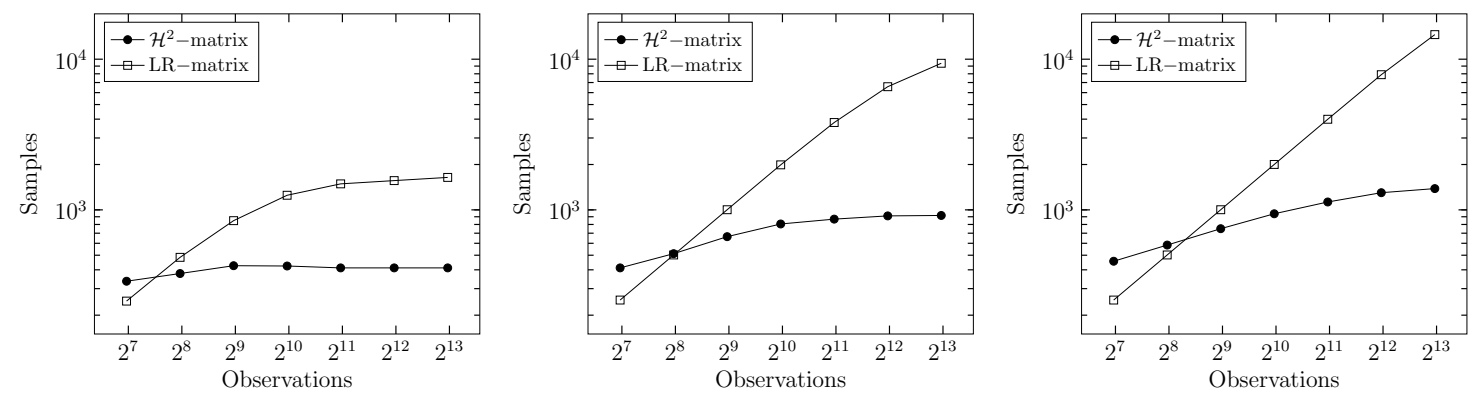

Figure 9.4: The $y$-axis shows the number of Hessian-vectors products (samples) needed for constructing hierarchically and globally low rank approximations of the misfit Hessian to an accuracy of $\epsilon=10^{-4}$ for increasing number of observations plotted on the $x$-axis. Shows are results for $\kappa=10^{-1}$ (left), $10^{-2}$ (middle), and $10^{-3}$ (right).

tational cost, I only consider the misfit Hessian, i.e., neglecting the Hessian of the regularization term. This allows the comparison of hierarchical matrix approaches with the more commonly used global low rank approach, which would suffer from inclusion of the Hessian of the regularization, which typically has full rank. Note that since this is a linear inverse problem, this misfit Hessian does not depend on the parameter $m$. Figure 9.4 shows the number of Hessian-vector products needed for constructing the misfit Hessian to a relative spectral accuracy of $10^{-4}$ for three different values of the diffusion coefficient $\kappa$ and an increasing number of observations. For small numbers of observations, the misfit Hessian has a rapidly decaying spectrum and a globally low rank representation can be achieved at a lower cost than with the hierarchical approach, measured in the number of Hessian-vector products. However, as more observations are incorporated, the Hessian applications required for the global low rank approximation grows rapidly. In contrast, the cost for the hierarchical compression only increases mildly with the number of observations as it is insensitive to the global rank. I also observe that larger diffusion coefficients $\kappa$ limit the increase of computational cost for increasing number of observations for both the hierarchical and the global low rank approximations. This is a consequence of the fact that diffusion limits the amount of fine-scale information that can be recovered 
in the inversion, and thus more observations do not have a significant effect on the rank of the misfit Hessian.
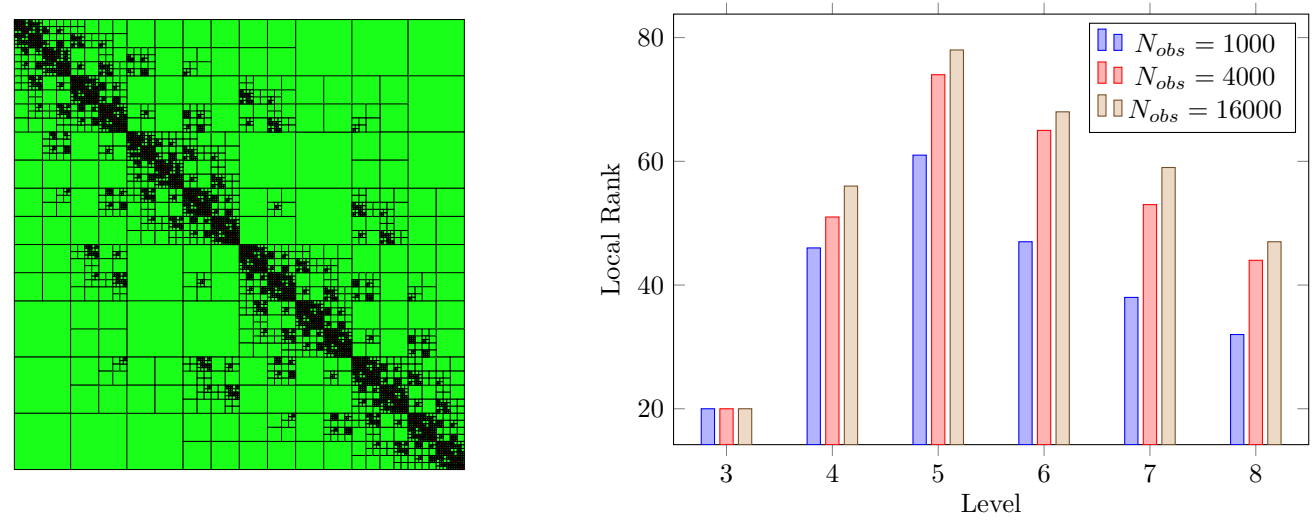

Figure 9.5: 8-level structure of Hessian (left) and corresponding maximum local ranks per level for different number of observations and $\kappa=10^{-3}$ (right).

The relative insensitivity of the number of samples to the data dimension is reflected in the fact that the blocks of the resulting $\mathcal{H}^{2}$ have small local ranks that do not grow much even as the global rank of the matrix increases. The left panel of Figure 9.5 shows the structure of the constructed matrix which uses a node ordering of the mesh obtained from a KD-tree binary space partitioning method, with leaf clusters of size 64 , and an admissibility condition that resulted in the refined matrix partitioning shown. The right panel of Figure 9.5 shows how the block ranks grow much more slowly than the global Hessian rank as the data dimension of the problem grows. 


\section{Chapter 10}

\section{Application: Hierarchical Matrix Approximations for Space-Fractional Diffusion Equations}

In this chapter, I show the effectiveness of hierarchical matrices in representing discretizations of non-local continuum models. Nonlocal continuum models, expressed as fractional differential equations, have gained significant popularity in recent years, as they have shown great success in representing the behavior of a variety of systems in scientific and engineering application domains [107]. Nonlocal models allow the representation of solutions that exhibit more singular and anomalous behavior than is possible with local continuum PDE models, which have long been the mainstay of scientific and engineering modeling. One of the most successful fractional differential equations is perhaps the space-fractional diffusion equation, which uses a fractional Laplacian operator that generalizes its classical counterpart. This fractional diffusion equation appears as a canonical model for transport problems involving anomalous diffusion that are of great relevance to industrial and environmental applications [108]. Applications from many other disciplines including image processing, finance, and many others [109, 110, 107] have also come to rely on fractional Laplacian operators. In its simplest form, the equation assumes a uniform diffusivity coefficient in space and a constant fractional order derivative, however non-homogeneous coefficients in general geometries and varying fractional orders are also of practical interest.

Numerical approximations for the fractional derivatives that appear in the fractional diffusion equations have received much attention in the literature [111]. Many different equivalent definitions of the fractional Laplacian have been presented [112] 
and used as the starting point for appropriate discretizations of the fractional operator. Finite-difference and variational methods are often adopted to numerically discretize the equations [113, 114, 115]. Particle-based methods, using random walk approaches [116, 117, 118, have also been used to simulate the equations. Smoothed particle approximations have been recently proposed [119] and shown to be quite flexible in discretizing and simulating the fractional diffusion equations.

The practical discretization schemes proposed, however, share serious computational difficulties that limit their scalability. The resulting discrete operator they produce is fully dense due to the nonlocal nature of the underlying equations and hence requires $O\left(N^{2}\right)$ memory and $O\left(N^{2}\right)$ operations for the core matrix vector multiplication operation. For simple Cartesian geometries with constant coefficients, translation invariance characteristics can be exploited [120] to reduce the storage and operator application costs. However, the general setting inevitably leads to a quadratic growth in computational resource requirements. This must be tamed if the simulation of fractional diffusion, or other fractional differential equations, is to be done at scale. Hierarchical matrix approximations have been proposed as algebraic representations of the discretized fractional operators. In 1D, $\mathcal{H}$-matrix approximations [121] were shown to be effective. The $\mathcal{H}$-matrix approximability of the discrete operators in two spatial dimensions was studied in [122, 123] starting from finite difference approximations of the fractional operator.

In this chapter, I start from a smoothed particle discretization of the fractional diffusion operator and construct asymptotically optimal $\mathcal{H}^{2}$-matrix approximations of the resulting matrix operator. I show that the memory requirements of the discretized fractional operator grow only as $O(N)$ where $N$ is the number of particles in the discretization, and that the cost of matrix-vector multiplication also grows at the same optimal linear rate. In addition, the constants that appear in these complexity estimates depend on the ranks of blocks in the matrix, which grow only very weakly 
with the desired accuracy of the matrix approximation, $k \sim \log |\epsilon|^{c}$ where $k$ is a representative rank of low rank blocks of the $\mathcal{H}$ matrix, $\epsilon$ is the accuracy of the hierarchical matrix relative to the fully dense representation, and $c$ is a constant that depends on the spatial dimension of the problem. The numerical experiments, with different problem sizes and fractional orders, show the broad applicability of $\mathcal{H}^{2}$-matrix representations for fractional diffusion problems.

In practice, I show that these $\mathcal{H}^{2}$ representations are effective in multiple spatial dimensions, not only in their optimal asymptotic growth, but importantly in their practicality on modern workstations. The operators can be compressed by more than

3 orders of magnitude relative to a dense representation, and generated in under a couple of minutes, to reasonably high accuracy, on problems of size $2 \mathrm{M}$ on a standard scientific workstation. I also show that a higher order variant of Newton-Schulz iteration can be used to generate an approximate inverse of the discretized operator, which can be used as an effective preconditioner for the fractional elliptic Poisson problem.

\subsection{The Fractional Diffusion Equation and its Particle Ap- proximation}

In this section, I briefly outline the formulation of the mathematical problem, and its particle approximation.

\subsubsection{The Fractional Laplacian}

I shall focus on the simulation of the multi-dimensional fractional diffusion equation,

$$
\frac{\partial u}{\partial t}=-\nabla \cdot \mathbf{Q}^{\beta}
$$


where

$$
\mathbf{Q}^{\beta}(\mathbf{x}, t) \equiv-\mathcal{D} \nabla^{\beta} u(\mathbf{x}, t)=-\mathcal{D} \frac{2^{\alpha-1} \Gamma\left(\frac{d+\alpha}{2}\right)}{\pi^{\frac{d}{2}} \Gamma\left(\frac{2-\alpha}{2}\right)} \int_{\mathbb{R}^{d}} \mathrm{pv} \frac{\mathbf{y}}{|\mathbf{y}|^{\alpha+d}} u(\mathbf{x}+\mathbf{y}, t) \mathrm{d} V(\mathbf{y}),
$$

is the fractional diffusion flux of order $\beta \in(0,1), \alpha \equiv \beta+1, \mathbf{x}, \mathbf{y} \in \mathbb{R}^{d}$ with $d \geq 1$, $\mathrm{d} V(\mathbf{y})$ is the measure in $\mathbb{R}^{d}, \mathcal{D}$ is the diffusivity, $\boldsymbol{\nabla}^{\beta}$ denotes the fractional gradient operator of order $\beta$, and "pv" indicates a principal value integral.

Assuming $\mathcal{D} \equiv 1$, 10.1 reduces to:

$$
\frac{\partial u}{\partial t}=\Delta^{\alpha} u
$$

where $\Delta^{\alpha} u$ is the fractional Laplacian operator of order $\alpha$ [124]:

$$
\Delta^{\alpha} u=\frac{\alpha 2^{\alpha-1} \Gamma\left(\frac{d+\alpha}{2}\right)}{\pi^{\frac{d}{2}} \Gamma\left(\frac{2-\alpha}{2}\right)} \int_{\mathbb{R}^{d}} \mathrm{pv} \frac{u(\mathbf{y})-u(\mathbf{x})}{|\mathbf{y}-\mathbf{x}|^{d+\alpha}} \mathrm{d} V(\mathbf{y})
$$

Note that in some of the literature the fractional Laplacian is denoted as $\Delta^{\alpha / 2}$. Also

note that in the limit $\alpha \rightarrow 2$, the fractional Laplacian $\Delta^{\alpha}$ defined in (10.4) coincides with the classical Laplacian.

For an infinite domain with no internal boundaries, the fundamental solution of (10.4) is available in integral form [124] for the general $d$-dimensional case.

\subsubsection{Particle Approximation}

Smooth particle methods consist in discretizing the $n$-dimensional spatial domain using a finite set of particles. The $i$-th particle is defined by its position $\mathbf{x}_{i}$, volume $V_{i}$, and strength $u_{i}$. The particle representation of a function, $u(\mathbf{x})$, is expressed as [125, 126, 127]:

$$
u(\mathbf{x}) \approx u_{\varsigma}(\mathbf{x}) \equiv \sum_{i \in \mathcal{I}} V_{i} u_{i} \eta_{\varsigma}\left(\mathbf{x}-\mathbf{x}_{i}\right), \quad \eta_{\varsigma}(\mathbf{x})=\frac{1}{\varsigma^{d}} \eta\left(\frac{\mathbf{x}}{\varsigma}\right)
$$


where $\mathcal{I} \subseteq \mathbb{Z}$ is a finite index set, $\eta$ is a radial kernel of unit mass, and $\varsigma$ is the so-called smoothing parameter. I adopt the second-order exponential kernel [128, 129]:

$$
\eta(\mathbf{x})=\frac{1}{\pi^{\frac{d}{2}}} \exp \left(-|\mathbf{x}|^{2}\right)
$$

The fractional diffusion flux is estimated by estimating the fractional Laplacian of (10.5) analytically. A midpoint rule to estimate the evolution of the particle strength leads to:

$$
\frac{\partial u_{i}}{\partial t}=\frac{1}{\varsigma^{\alpha}} \sum_{j \in \mathcal{I}} V_{j} u_{j} G_{\varsigma}\left(\mathbf{x}_{i}-\mathbf{x}_{j}\right)
$$

where

$$
G_{\varsigma}(\mathbf{r})=\frac{1}{\varsigma^{d}} G\left(\frac{\mathbf{r}}{\varsigma}\right), \quad G(\mathbf{r})=-\frac{2^{\alpha} \Gamma\left(\frac{\alpha+d}{2}\right)}{\pi^{\frac{d}{2}} \Gamma\left(\frac{d}{2}\right)}{ }_{1} F_{1}\left(\frac{\alpha+d}{2}, \frac{d}{2},-|\mathbf{r}|^{2}\right)
$$

and ${ }_{1} F_{1}$ denotes the confluent hypergeometric function of the first kind [130]. For additional details regarding the construction, see [119].

This discretization scheme can be written as a matrix system,

$$
\dot{\mathbf{U}}=A \mathbf{U}
$$

where $\mathbf{U}$ is the vector representing the particle strengths, $u_{i}, \dot{\mathbf{U}}$ is its time derivative, and $A$ is a matrix with elements

$$
A_{i, j}=\frac{1}{\varsigma^{\alpha}} V_{j} G_{\varsigma}\left(\mathbf{x}_{i}-\mathbf{x}_{j}\right)
$$

Note that the computation of each entry, $A_{i, j}$, requires the evaluation of a confluent hypergeometric function, which is computationally demanding [131]. However, if the particle grid is held fixed, evaluation of the diffusion matrix, $A$, may be per- 
formed once. Even if the diffusion is estimated using a fixed particle grid however, a significant challenge arises because the matrix $A$ is full. This leads to an $\mathrm{O}\left(N^{2}\right)$ storage requirement, where $N$ is the total number of particles. This storage requirement can become prohibitively large, even for a relative modest computational grid. For instance, with particles uniformly distributed on a 2D grid with 301 cells in each direction, storage of the matrix in double precision would require about $61 \mathrm{~GB}$ of RAM. Even though this storage requirement could potentially be avoided by directly computing the local diffusion terms individually, such direct computation would necessitate an $\mathrm{O}\left(N^{2}\right)$ operation cost. Thus, one is generally faced with potentially prohibitive memory and CPU requirements, unless the drawbacks of the direct matrix representation and source term evaluation are suitably addressed. Hierarchical matrices are an ideal representation for this purpose, as shown in the rest of this chapter.

\subsection{Interpolatory Construction}

In order to construct the hierarchical matrix representation of the discrete operator, I start by clustering the $N$ particles into small regions with clusters of cardinality $\leq n_{\min }$ using a $k$-d tree binary partitioning procedure. Figure 10.1 shows the resulting clusters for two sample particle discretizations of a square and a disk. The $k$-d tree partitioning procedure generates a hierarchy of clusters that define the block rows and block columns of the hierarchical matrix at different levels of granularity, ending at the leaf level clusters. The labels $t$ and $s$ of Figure 10.1 refer to sample clusters in these hierarchies. Each such pair will generate the block $A_{t s}$ of the matrix. The cluster tree together with an admissibility criterion define the matrix structure. A construction algorithm needs to generate: (1) the leaf-level bases $U_{t}$ for all leaf clusters $t,(2)$ the corresponding interlevel transfer matrices $E_{t}$ for all clusters at all levels, and (3) the matrix blocks $S_{t s}$ for all low rank blocks. 

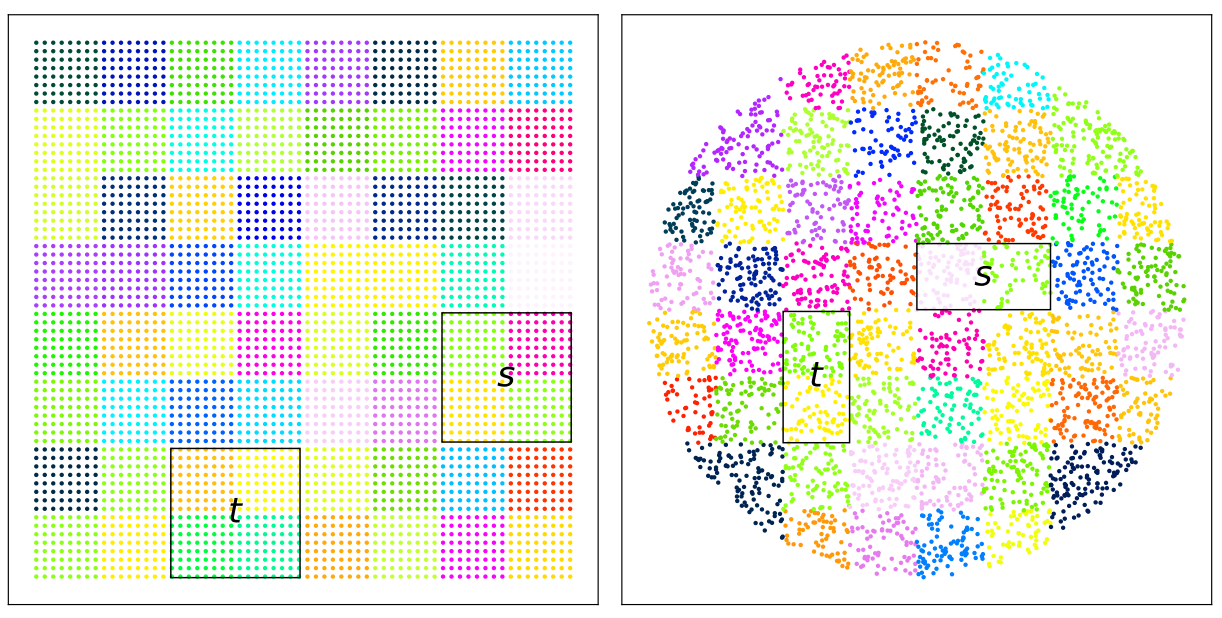

Figure 10.1: Clustering produced by a binary $k$-d tree partitioning for a regular (left) and irregular (right) spatial discretization of square and circular regions. The labels $t$ and $s$ identify the clusters that affect a matrix block $A_{t s}$.

The basic element of the construction consists of tensor product grids of Chebyshev interpolation points of size $m^{d}$ that are overlaid on axis-aligned bounding boxes $\Omega_{t}$ and $\Omega_{s}$ of clusters $t$ and $s$, respectively. This construction is used to build Lagrange polynomials $p(x)$ of order $d m$ over $d$-dimensional boxes. Chebyshev points have a well-known best approximation property for representing smooth functions. The core observations for constructing the nested basis hierarchical matrix are (1) that the kernel function can be written as a separable approximation:

$G\left(x_{t}, x_{s}\right) \approx \tilde{G}\left(x_{t}, x_{s}\right)=\sum_{i} \sum_{j} S_{i j} p_{t, i}\left(x_{t}\right) p_{s, j}\left(x_{s}\right), \quad x_{t} \in \Omega_{t}, x_{s} \in \Omega_{s}$, and $i, j=1: m^{d}$

where $p_{t, i}$ and $p_{s, j}$ are the $i$-th and $j$-th basis polynomials over $\Omega_{t}$ and $\Omega_{s}$, and (2) that the $m^{d}$ polynomials $p_{t, i}$ used in the approximation over a region $\Omega_{t}$ are expressed in terms of the approximating polynomials over the subregions of children clusters $t_{c}$ $\left(t=\cup_{c} t_{c}\right)$

$$
p_{t, i}(x)=\sum_{k}\left(E_{t_{c}}\right)_{k, i} p_{t_{c}, k}(x), \quad x \in \Omega_{t_{c}}, \quad k=1: m^{d} \text {, and } c=1,2
$$


Based on these interpolation points, the elements of the hierarchical matrix approximation may be directly evaluated. The leaf-level bases $U_{t}$ are of size $n_{t} \times k_{t}$, where $n_{t}$ is the cardinality of cluster $t$ and $k_{t}=m^{d}$ is the number of points in the interpolation grid. Their columns can be computed by evaluating the basis polynomials at the $n_{t}$ points of $t$. The interlevel transfer matrices $E_{t_{c}}$ for a child cluster $t_{c}$ with parent cluster $t$ can also be computed column by column by simply evaluating the basis polynomial of $\Omega_{t}$ at the interpolation points of $\Omega_{t_{c}}$. Finally, the $i j$ entries of the small $S_{t s}$ matrices can be computed by evaluating the kernel function at the $i$-th interpolation point of $\Omega_{t}$ and the $j$-th interpolation point of $\Omega_{s}$.

One question to address concerns the best values of $m$ (which may vary from cluster to cluster as well from level to level) needed in order to reach a desired target approximation accuracy, $\epsilon$, in the matrix. The order, $m$, used in the Lagrangian interpolation directly affects the error $\epsilon_{G}=\| G\left(x_{t}, x_{s}\right)-\tilde{G}\left(x_{t}, x_{s} \|\right)$ in the polynomial approximation of the kernel function. For smooth kernel functions the convergence of the approximating polynomial is exponential in $m$. It can also be shown that the error in the resulting hierarchical matrix approximation, measured either by the spectral or Frobenius norms, is also directly related to $\epsilon_{G}$ [132. Unfortunately, the constants that appear in the matrix error estimates depend on the geometric sizes and shapes of the clusters and their relative position and orientations, and the bounds are not always tight. Therefore my strategy for constructing the matrix approximation is to use a conservative value, $m$, that first generates a tighter error than desired (and therefore uses more memory in the approximation of $A$ ), and then compress it algebraically to the desired tolerance, $\epsilon$, using the methods of Chapter 5 to obtain the optimal approximation. 


\subsection{Performance of Hierarchical Matrix Operations}

In this section, I demonstrate the efficiency of the hierarchical matrix operations on both CPU and GPU machines. For these experiments I use a set of $N$ particles placed at the nodes of a regular $n \times n$ grid in order to assess the scalability, in both time and memory, of the hierarchical matrix computations with problem size. In all experiments, the matrix structure is constructed by hierarchically clustering the $N$ points using a $k$-d tree data structure. The $k$-d tree partitioning algorithm subdivides the point set until a leaf size of $n_{\min }=64$ is reached. A pair of clusters $t$ and $s$ at level $l$ of the hierarchy generates a low rank matrix block $A_{t s}^{l}$ if the inter-center distance between the point sets is large relative to their average size, $\left\|\mathbf{x}_{t}-\mathbf{x}_{s}\right\|>\eta\left(D_{t}+D_{s}\right) / 2$. In this algebraic admissibility condition, $\mathbf{x}$ represents the location of the center of the cluster, $D$ the diameter of a ball enclosing it, and $\eta$ is a tuning parameter of the condition that eventually has the effect of controlling the block refinement of the resulting hierarchical matrix. I fixed $\eta=0.8$ for these experiments, and used a fractional order $\alpha=1.5$. The resulting matrix structure for a problem size $N=16,384$ is shown in Figure 10.2 .

Figure $10.3 \mathrm{a}$ shows the performance of the two phases of the construction of the discrete operator as well as the overall time needed to produce the final compressed matrix. For the first phase, as described in Section 10.2, I use a constant rank of 100 for the interpolation to achieve an overall accuracy of about $10^{-6}$ which I verify by sampling $5 \%$ of the entries of the output of the full dense matrix with a random vector and comparing against the output of the hierarchical matrix vector product. The resulting storage requirement of the low rank portion of the matrix grows linearly with the problem size, but it is still relatively high. The second phase, as described in Chapter 5, performs algebraic compression to an accuracy of $10^{-5}$ to greatly reduce the overall memory footprint. Both phases have linear asymptotic complexity which is seen in Figure 10.3a. The memory consumption of the dense $\left(A_{d}\right)$ and low rank 


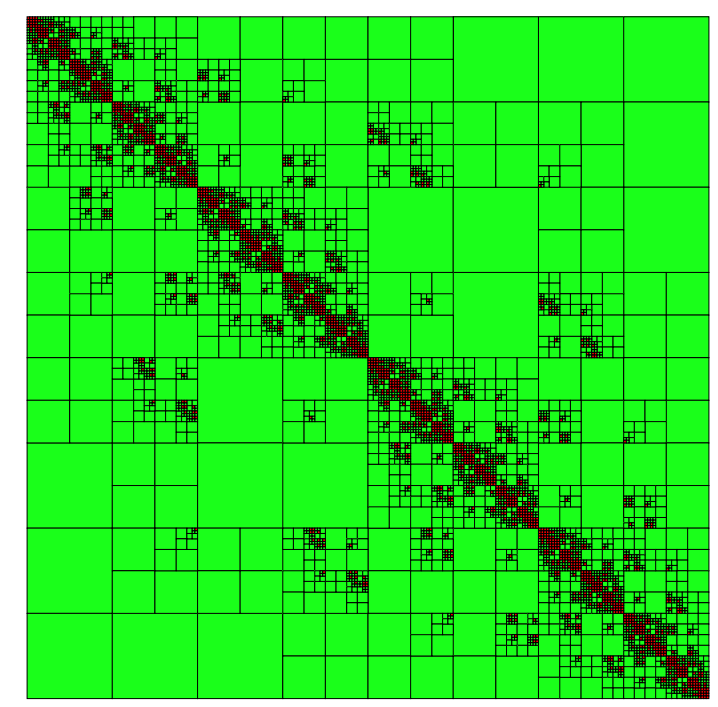

Figure 10.2: Hierarchical Matrix Structure produced by admissibility parameter $\eta=$ 0.8 for a regular $2 \mathrm{D}$ grid.

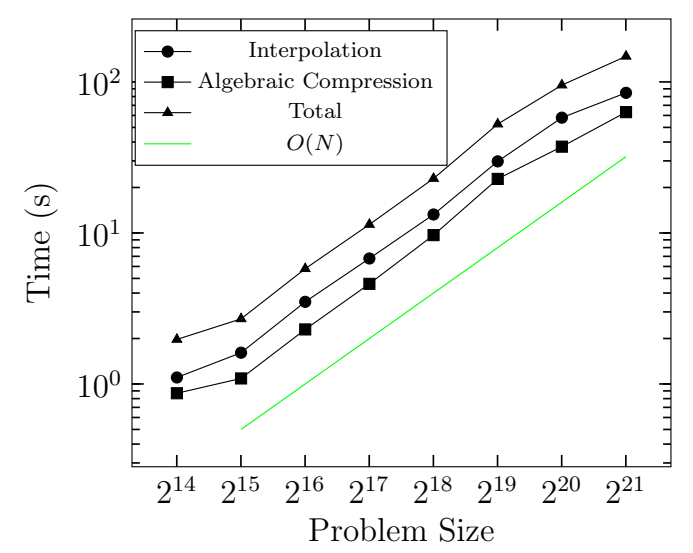

(a) CPU time for the two phases of the construction.

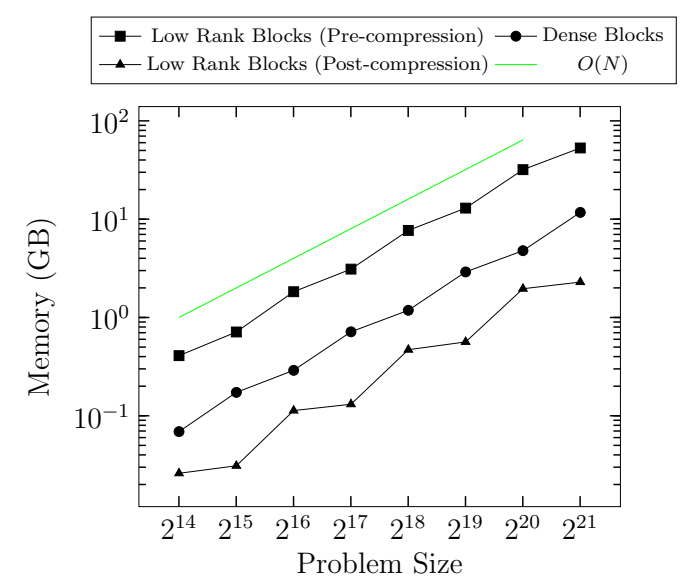

(b) Memory consumption and effect of algebraic compression on low rank blocks memory.

Figure 10.3: Performance of matrix construction to an error threshold of $10^{-5}$ on a range of problem sizes: (a) CPU time for interpolation and algebraic compression phases; (b) Memory footprint of the dense and low rank blocks of the resulting matrix (before and after algebraic compression). 
174

\begin{tabular}{|c|c|c|c|}
\hline $\mathrm{N}$ & $\mathcal{H}^{2}$ Memory & Dense Memory & Memory Ratio \\
\hline \hline 16384 & 0.095 & 2 & 21 \\
\hline 32768 & 0.204 & 8 & 39 \\
\hline 65536 & 0.403 & 32 & 79 \\
\hline 131072 & 0.846 & 128 & 151 \\
\hline 262144 & 1.653 & 512 & 310 \\
\hline 524288 & 3.471 & 2048 & 590 \\
\hline 1048576 & 6.743 & 8192 & 1215 \\
\hline 2097152 & 14.01 & 32768 & 2339 \\
\hline
\end{tabular}

Table 10.1: The memory consumption in GB and memory savings of using a hierarchical representation instead of a dense one.

$\left(A_{l r}\right)$ portions of the matrix are shown in Figure $10.3 \mathrm{~b}$. The reduction in memory footprint achieved by the algebraic compression can be seen by comparing the two low rank curves in Figure 10.3b. A factor of more than 20x reduction in storage for the low rank blocks can be achieved on the larger problem sizes.

Table 10.1 also shows the total savings in memory of the hierarchical matrix (dense blocks plus low rank blocks) computed to an overall accuracy of $\epsilon=10^{-5}$ when compared with a full dense matrix representation. The linear growth in memory footprint of the $\mathcal{H}^{2}$ hierarchical representation stands in sharp contrast to the quadratic growth of the dense representation. This is, principally, why these hierarchical matrices have the potential for being the basic computational engine of fractional diffusion simulations at scale.

Figure 10.4a shows the effect of increasing the compression threshold $\epsilon$ on the low rank memory consumption. As expected there is a weak logarithmic dependence of the memory footprint on the accuracy of the approximation. A growth of about $O\left(|\log \epsilon|^{2}\right)$ is observed. This translates to an almost tenfold increase in low rank block storage for an increase of 4 digits of accuracy from an initial 2 digits when compressing a matrix of size $2^{20}$. The memory footprint of the dense blocks is obviously not affected. Figure $10.4 \mathrm{~b}$ shows the maximum block rank for every level of the hierarchy in the matrix. The corresponding increase in the maximum ranks of each level of the 


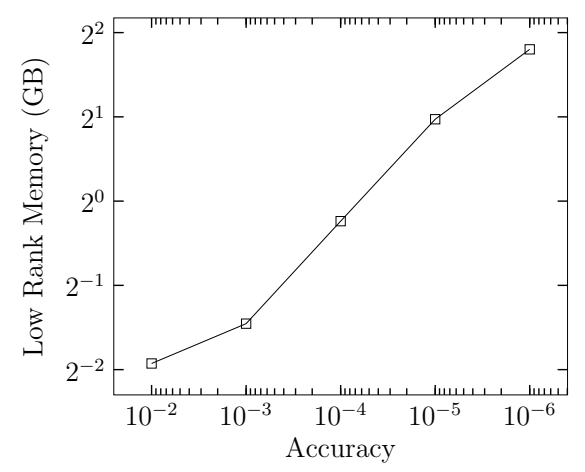

(a) Effect of the approximation accuracy on low rank storage.

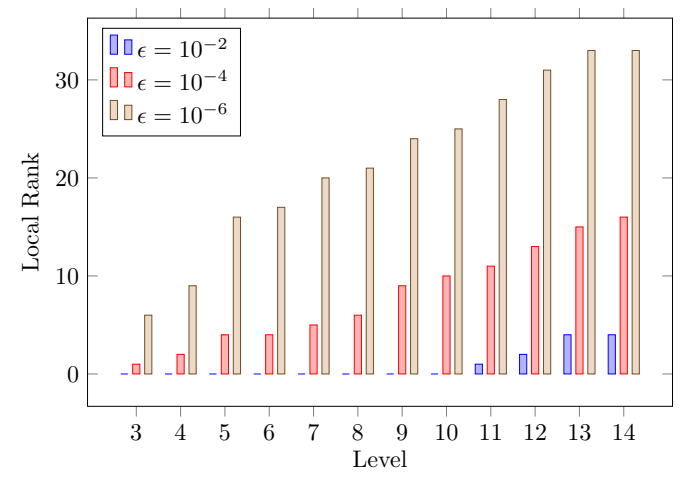

(b) Maximum block ranks for each level of the matrix for varying compression thresholds.

Figure 10.4: Impact of the choice of the compression threshold on the memory footprint and maximum block rank of a fractional diffusion hierarchical matrix of size $2^{20}$.

hierarchical matrix for compression thresholds of $10^{-2}, 10^{-4}$, and $10^{-6}$ also shows a weak logarithmic dependence on desired approximation accuracy, of about $O\left(|\log \epsilon|^{2}\right)$.

Once the matrix has been compressed, I can efficiently perform the necessary matrix vector products on both the CPU and the GPU. These experiments are performed on a 12-core workstation and a P100 NVIDIA Pascal GPU, respectively. Though the GPU memory is limited to $16 \mathrm{~GB}$, this does not pose any issues for problem sizes up to about two million, due to the greatly reduced storage requirements of the compressed matrix. Figure 10.5 shows the performance of the hgemv operation averaged over 10 runs, clearly demonstrating the linear complexity of the algorithm, and the high efficiency of the GPU in particular, which is able to perform the matvec operation in under $40 \mathrm{~ms}$ on a problem of size $2 \mathrm{M}$.

Using the hierarchical matrix compression and matrix vector product operations, I construct an approximate inverse and evaluate its effectiveness as a preconditioner for the conjugate gradient $(\mathrm{CG})$ method. The inverse of a regularized version of the discretized operator $(\tilde{A}=A+\sigma I)$ is constructed and used. I use an order 16 hyperpower iterative method to compute the approximate inverse as described in 


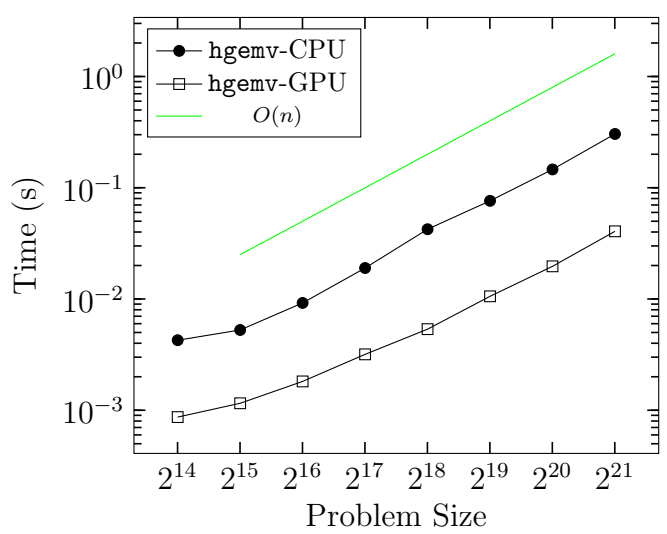

Figure 10.5: Performance of the matrix-vector multiplication.

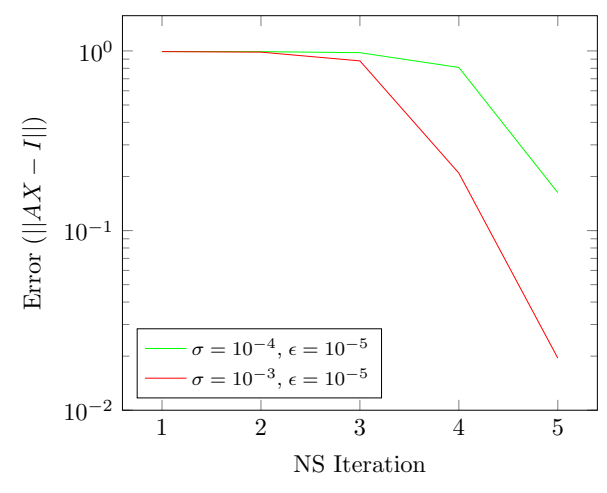

(a) Convergence rate of the order 16 hyperpower iterates for the regularized matrix.

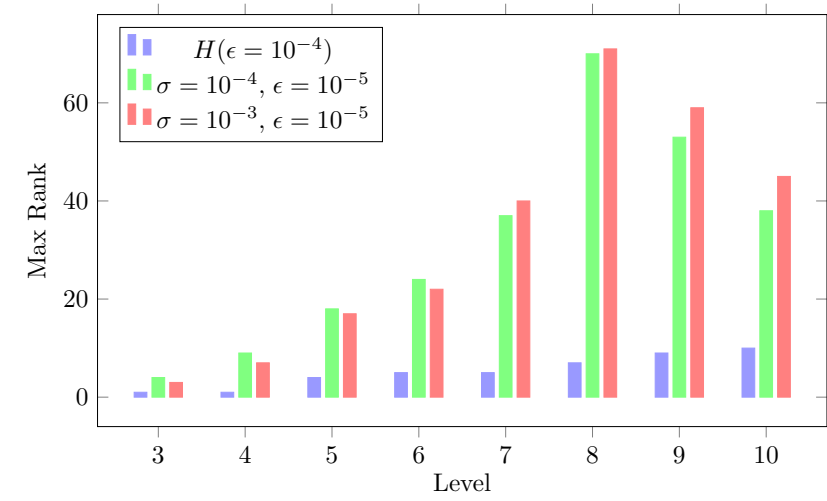

(b) Maximum block ranks at each level of the hierarchy for the final iterates, when compressed to a threshold of $10^{-5}$.

Figure 10.6: Performance of the order 16 hyperpower iterative inversion.

Chapter 7 and an approximation accuracy $\epsilon$ for the constructed iterates.

Figure 10.6a shows the convergence of the hyperpower iterates for a problem size of $2^{16}$ for two values of the regularization parameter $\sigma=\left(10^{-3}, 10^{-4}\right)$, where each iterate is constructed to a threshold of $\epsilon=10^{-5}$. The runtime per iteration depends heavily on the ranks of the iterates which tend to increase for the intermediate iterates. For example, the first iteration completes in about $56 \mathrm{~s}$, whereas the final iteration takes $763 \mathrm{~s}$, taking about $1771 \mathrm{~s}$ in total for the five iterations performed. Figure $10.6 \mathrm{~b}$ shows the maximum block ranks for each level of the hierarchy for the approximate inverses reached after five iterations. There is a noticeable growth in the block ranks 


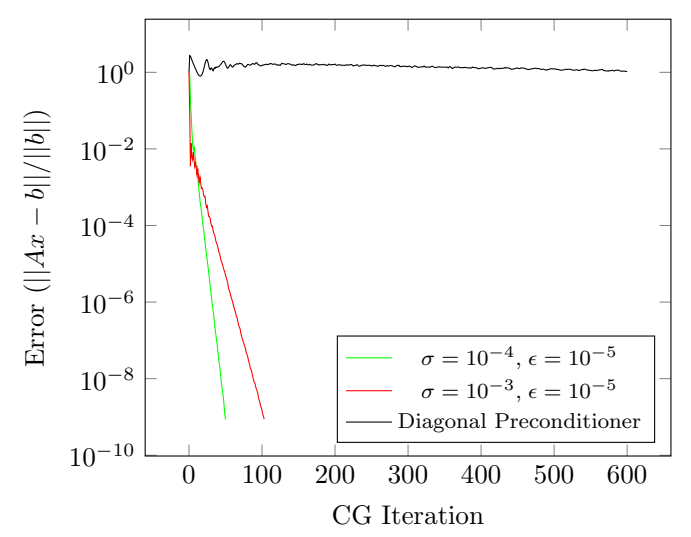

Figure 10.7: The convergence behavior of the preconditioned conjugate gradient method using a diagonal preconditioner and the two approximate (regularized) inverse preconditioners.

of the approximate inverse compared to the ranks of the original matrix.

Employing the resulting matrices as preconditioners for the solution of the system $A x=b$ using a CG method, I obtain the iteration counts of Figure 10.7. The righthand side vector used in the figure is a vector of all ones, $b=1$, with the solution started at $x_{0}=\mathbf{0}$, although comparable results were obtained for values of $b$ randomly sampled from the uniform $[0,1]$ distribution. I note that the diagonal preconditioner cannot produce a reliable solution, while the two approximate inverses obtained after five iterations of the iterative scheme $(7.2)$ are able to produce a solution robustly to high accuracy. Not unexpectedly, the approximate inverse with the smaller regularization term is more effective than the one with the larger regularization term when it comes to CG iteration count. In both cases, I note that a coarse approximation of the regularized inverse is sufficient for robust convergence.

\subsection{Illustrative Simulations}

In this section, I analyze the performance of the hierarchical solution algorithm in the context of two simulations. In the first simulation, I focus on the solution of the fractional diffusion equation in an unbounded 2D domain, and rely on the available 
analytical solution to test the approximation quality of the computed solution. This enables me to assess the impact of approximating the full diffusion matrix by its hierarchical counterpart, and also to analyze the computational gains achieved through this approximation. In the second simulation, I consider the solution of the forced fractional diffusion equation in a bounded domain, and carry out the integration for a sufficiently long interval so as to effectively reach a steady-state solution. This second setting explores the potential of applying the hierarchical matrix algorithms as means to obtain a (pseudo-transient) solution of a fractional elliptic problem. I also compare the pseudo-transient solution to that obtained by the CG method, preconditioned by a hierarchical approximate inverse, for solving directly the corresponding steady state fractional Poisson problem.

\subsubsection{Transient Solution}

In this section, I assess the performance of the hierarchical matrix operations based on the transient solution of the fractional diffusion equation 10.3$)$ in $\mathbb{R}^{2}$. I use as initial condition the fundamental solution at $t_{0}=0.5$ and carry out the integration until a final time $t_{f}=1.5$. The availability of the analytical solution and of numerical solutions computed with direct particle-particle interactions [119] provide a suitable setup to assess both performance, as well as errors associated with hierarchical approximation of the full diffusion matrix.

To provide a suitable spatial discretization throughout the period of integration, particles are distributed over a uniform grid covering a square domain of side $2 D$, where

$$
D=4 t_{f}^{1 / \alpha} R_{\alpha}
$$

and $R_{\alpha}$ is the characteristic length [119]. The grid size (inter-particle distance) is $h=2 D /\left(N_{x}-1\right)$, where $N_{x}$ is the number of points in the $x$ and $y$ directions. 


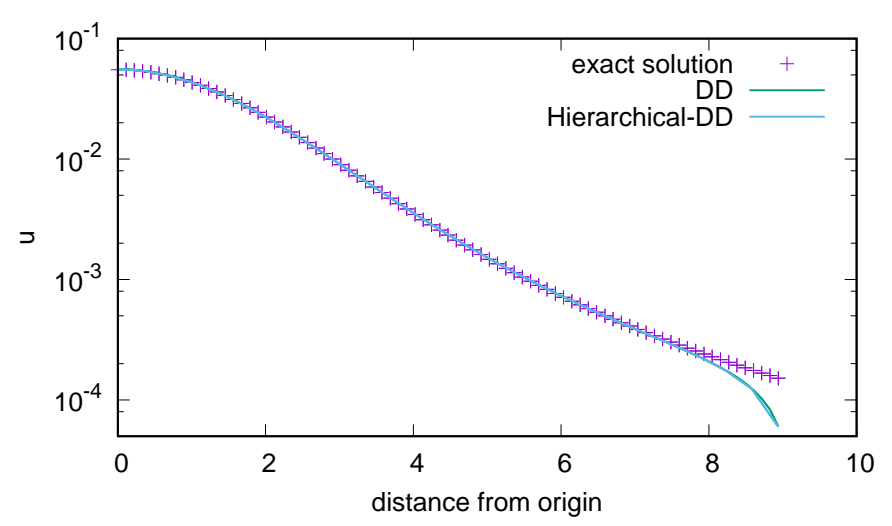

Figure 10.8: Fundamental solution at $t_{f}=1.5$ for $\alpha=1.5$. Also plotted are profiles obtained using the full diffusion matrix and its hierarchical approximation; in both cases $N_{x}=161$.

\section{Accuracy}

Figure 10.8 contrasts the analytical and numerical solutions at $t_{f}=1.5$ for the case $\alpha=1.5$. The numerical solutions are obtained using the full diffusion matrix with $N_{x}=161$ and $\varsigma=2 h$, and its hierarchical approximation. A good agreement with the exact solution is observed, though small but noticeable errors are observed near the boundaries of the truncated domain. As discussed in [119] the observed discrepancies are dominated by domain truncation effects. Figure 10.8 also shows that solutions obtained using the full and hierarchical diffusion matrices are in close agreement throughout the domain. This suggests that the hierarchical matrix representation does not appreciably affect the quality of the computed solution.

To further quantify prediction accuracy, as in [119] I estimate the $l_{1}$ error defined according:

$$
\varepsilon_{D_{\varepsilon}}=\frac{\sum_{i \in \mathcal{I}_{\epsilon}} V_{i}\left|u_{i}\left(t_{f}\right)-\mathcal{G}_{\alpha}\left(\mathbf{x}_{i}, t_{f}\right)\right|}{\int_{D_{\varepsilon}} \mathcal{G}_{\alpha}\left(\mathbf{x}, t_{f}\right) \mathrm{d} V(\mathbf{x})},
$$

where $D_{\varepsilon}$ denotes the subregion $[-5,5] \times[-5,5]$. Figure 10.9 shows estimates of $\varepsilon_{D_{\varepsilon}}$ for different orders ( $\alpha=1.1,1.5$, and 1.9) and grid resolutions $\left(N_{x}=41,81,161,321\right.$, and 641). In all cases, the regularization parameter $\varsigma=2 h$. The results indicate that 


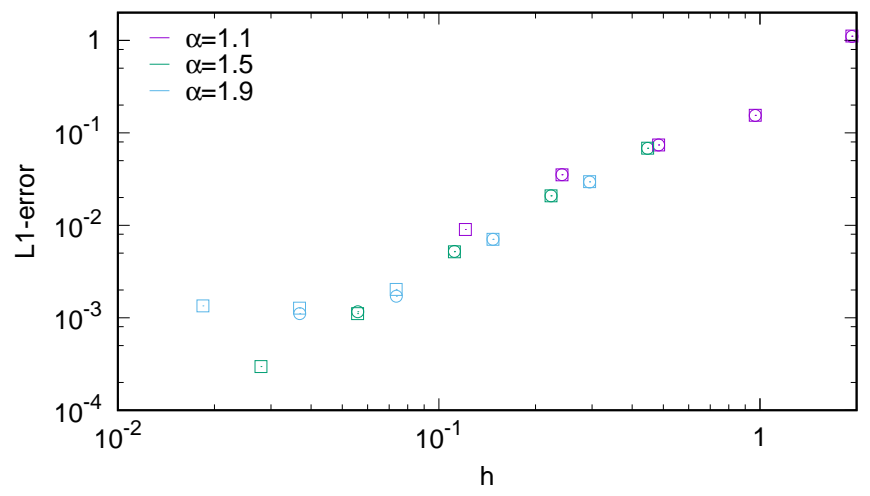

Figure 10.9: L1-error $\varepsilon_{D_{\varepsilon}}$ for different values of $\alpha$ and $h$, as indicated. Plotted are results obtained using the full diffusion matrix (circles) and its hierarchical approximation (squares).

the hierarchical approximation of the diffusion matrix has insignificant impact on the computed errors. In particular, the error in the case of the hierarchical algorithm also exhibits second-order behavior. Note that as $h$ decreases, estimates of $\varepsilon_{D_{\varepsilon}}$ tend to level off. As discussed in [119] this phenomenon occurs as discretization errors drop and approach domain truncation errors.

\section{Performance}

The performance gains achieved by the hierarchical algorithm are assessed in terms of the wall time required to construct the hierarchical matrix representation, the time required to integrate the governing equation, and the memory requirements.

Table 10.2 reports computational times and workspace requirement estimates for full and hierarchical matrix computations. Provided are results obtained for different values of $N_{x}$ and $\alpha$. It is clear when the system size is small $\left(N_{x}=41\right.$ and 81$)$, application of the hierarchical representation algorithm is not attractive. This is the case because of the overheads incurred in forming the hierarchical representation, which in this parameter range dominate the relatively small gains achieved during the integration. However, as the system size increases, $N_{x} \geq 161$, the advantages of the hierarchical algorithms become substantial. Specifically, despite the effort 
Table 10.2: Computational cost and memory requirements of the full-matrix and the hierarchical-matrix computations. All the simulations were conducted on the same workstation, comprising 28 physical cores (56 in hyperthreading) on Intel CPU's, and 127 GB of RAM. The codes were designed to run in shared memory parallelism, and compiled using the same compiler.

\begin{tabular}{|c|c|c|c|c|}
\hline \multirow[t]{2}{*}{$N_{x}$} & \multirow[t]{2}{*}{ DD } & \multicolumn{3}{|c|}{ Hierarchical DD } \\
\hline & & $\alpha=1.1$ & $\alpha=1.5$ & $\alpha=1.9$ \\
\hline \multicolumn{5}{|c|}{ Time to form the matrix $(\mathrm{s})$} \\
\hline 41 & 1 & 17 & 18 & 18 \\
\hline 81 & 1 & 17 & 18 & 18 \\
\hline 161 & 4 & 24 & 25 & 25 \\
\hline 321 & 30 & 49 & 49 & 50 \\
\hline 641 & - & 124 & 122 & 121 \\
\hline \multicolumn{5}{|c|}{ Time to integrate the system $(\mathrm{s})$} \\
\hline 41 & 0.2 & 4 & 4 & 4.1 \\
\hline 81 & 5.6 & 4.3 & 4 & 3.9 \\
\hline 161 & 96 & 18 & 16 & 15 \\
\hline 321 & 1700 & 76 & 70 & 66 \\
\hline 641 & - & 606 & 569 & 560 \\
\hline \multicolumn{5}{|c|}{ Required workspace (GB) } \\
\hline 41 & 0.021 & 0.014 & 0.014 & 0.013 \\
\hline 81 & 0.32 & 0.074 & 0.072 & 0.066 \\
\hline 161 & 5 & 0.341 & 0.329 & 0.297 \\
\hline 321 & 79.1 & 1.457 & 1.406 & 1.26 \\
\hline 641 & - & 6.041 & 5.82 & 5.205 \\
\hline
\end{tabular}

required to form the hierarchical matrix, the hierarchical algorithm results in net savings. The overall savings are about a factor of 2 at $N_{x}=161$; they amount to a factor of 14 approximately for $N_{x}=321$. Note that for $N_{x}=641$, I sm no longer able to form the full diffusion matrix, as storage of the full matrix would require an estimated $1250 \mathrm{~GB}$ of RAM. In contrast, the workspace required by the hierarchical representation remains at a reasonable level, as it is seen to grow linearly with system size $\left(N_{x}^{2}\right)$. The efficiency gains can also be appreciated by noting that the hierarchical simulation at $N_{x}=641$ required a fraction of the time needed to perform the full matrix simulation at the coarser resolution level $\left(N_{x}=321\right)$. In light of the present experiences, the hierarchical representation can be viewed as an enabling feature for extending the present fractional diffusion algorithms to large systems in multiple 
dimensions.

\subsubsection{Forced Transient and Steady State Solutions}

In this section, I apply the hierarchical matrix representation to compute the evolution solution of the forced, fractional diffusion equation:

$$
\frac{\partial u}{\partial t}=\Delta^{\alpha} u-q(\mathbf{x})
$$

in the square domain $\mathcal{D}=(-D, D) \times(-D, D)$, with initial condition $u(\mathbf{x}, 0)=0$, and boundary condition $u(\mathbf{x}, t)=0$ for $\mathbf{x} \notin \mathcal{D}$. Here $q(\mathbf{x})$ is a given steady source term.

I also use a CG method, preconditioned by an approximate hierarchical inverse, to solve directly the fractional elliptic problem:

$$
\Delta^{\alpha} u=q(\mathbf{x})
$$

\section{Transient Solution}

I first carry out the simulations to approximate the steady solution of 10.15 , and thus apply the present setting as a pseudo-transient approach to simulate the solution of 10.16 .

I rely on a uniform particle grid with size $h=2 D /\left(N_{x}-1\right)$. I set $D=4$, and $q$ to be a Gaussian pulse with zero mean and unit variance.

Let $\mathbf{U}$ be the vector of particle strengths, and let $\mathbf{q}$ denote the corresponding source term vector. Applying a first-order explicit time discretization of $(10.15)$ leads to the following evolution equation for the particle strengths

$$
\frac{\mathbf{U}^{s+1}-\mathbf{U}^{s}}{\Delta t}=A \mathbf{U}^{s}-\mathbf{q}
$$

where the superscript denote the time level. 


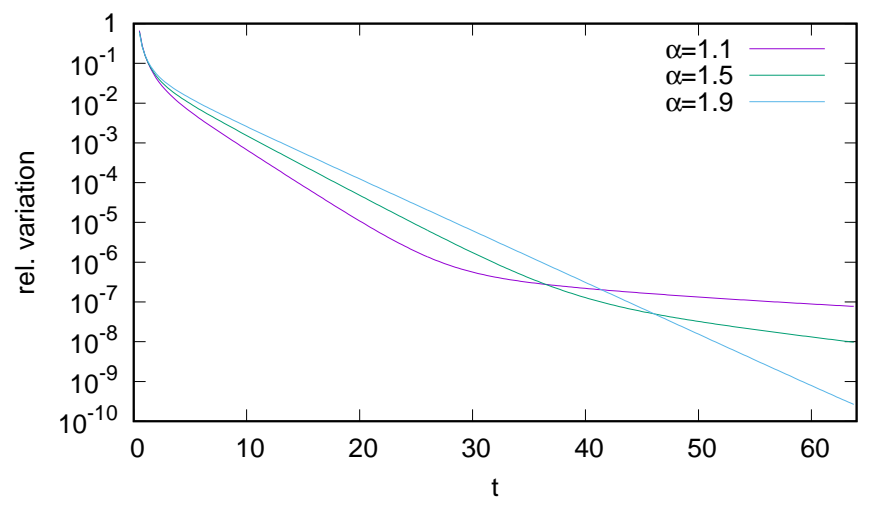

Figure 10.10: Relative variation in time of the solution at $\mathbf{x}=0$ and different values of $\alpha$ as indicated. The simulations are performed using $N_{x}=161$ and $\Delta t=2.5 \cdot 10^{-3}$.

I consider three values of the fractional order, namely $\alpha=1.1,1.5$, and 1.9, and carry out the computations up to $t_{f}=64$. For all considered values $\alpha$, this final time is sufficiently large for the solution to become essentially steady. To verify that this is the case, I estimate the relative change in time of the solution at the origin

$$
\delta u^{s+1}=\frac{u^{s+1}(x=0, y=0)}{u^{s}(x=0, y=0)}-1 .
$$

Results are plotted in Fig. 10.10. At early times, the evolution of the solution near the origin is dominated by the source term, and so the curves for different $\alpha$ are almost identical. When the diffusion becomes significant, the evolution exhibits substantial dependence of $\alpha$. As I approach $t_{f}$, in all cases the relative change becomes very small, showing that the solution can be considered as essentially steady.

Figure 10.11 illustrates the computed solutions at the final for all considered values of $\alpha$. The simulations are conducted using a grid with $N_{x}=161$, i.e., with $N=25,921$ particles. The time integration uses a step size $\Delta t=2.5 \cdot 10^{-3}$, selected within the stability range of the explicit integration scheme [119]. Despite the large number of integration steps $(25,600)$, implementation of the hierarchical scheme enables me to estimate the steady solution efficiently. Examination of the results 


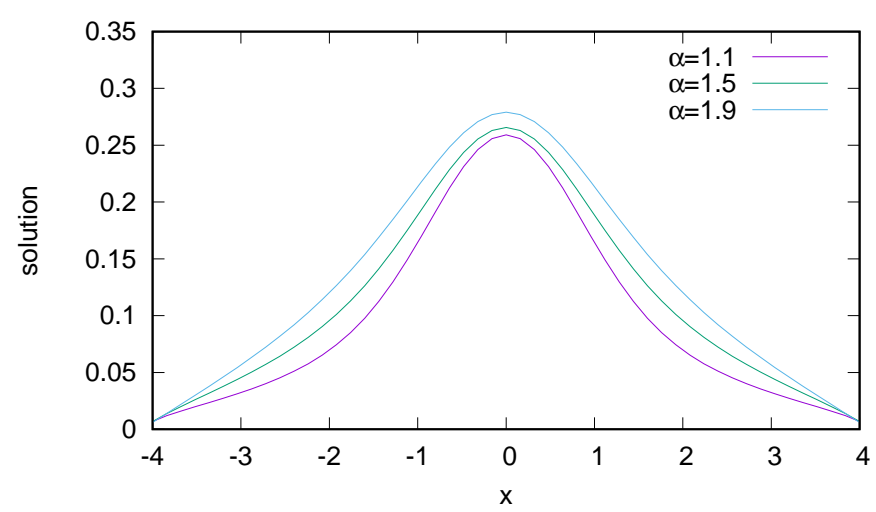

Figure 10.11: Profile of the computed solution at $t=64$ along $y=0$. Plotted are curves generated for different values of $\alpha$, as indicated.

(not shown) indicates that in all cases the solution remains radially symmetric, as expected. Consequently, the depicted axial profiles provide a full characterization of the predictions. In all cases, a smooth solution is obtained, with a peak at $\mathbf{x}=0$.

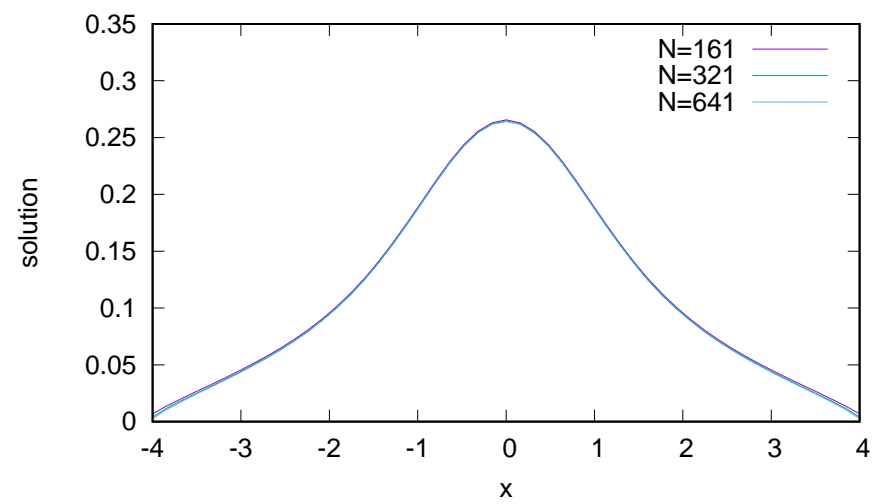

Figure 10.12: Profile of the computed solution at $t=64$ along $y=0$. Plotted are curves generated $\alpha=1.5$, and different values of $N_{x}$, as indicated.

To examine the dependence of the computed solution on the resolution, I repeat the same experiment for a higher number of grid points, $N_{x}=321$ and 641 . For brevity, I only report in Fig. 10.12 results obtained for $\alpha=1.5$. The plot indicates that for all values of $N_{x}$, the solution are nearly identical over most of the computational domain, whereas small differences are discernible near the boundaries. Note that the boundary condition is not exactly satisfied, which is in fact anticipated because a simplified treatment of boundary condition is adopted, specifically based on setting 
to zero the strength of particles falling outside the domain. Because a smoothing function is used, the boundary condition is not exactly achieved. However, as the discretization is refined, the predictions at the boundary are seen to drop towards zero. The rate of convergence of the solution is estimated to be approximately 1 . Thus, in the present case, though it is evidently accurate in the interior, the scheme is effectively behaving as first order. This is also expected because in a bounded domain, solutions of the fractional elliptic equations are generally not smooth at the boundary (e.g., [133]).

\section{Steady State Solution}

Finally, I consider the behavior of a CG method for solving the steady state equations 10.16 for the same fractional order values $\alpha=1.1,1.5$, and $1.9, N_{x}=161$, and the same problem parameters $D$ and $q$ used above. The hierarchical discrete operator $A$ is generated to an accuracy of $10^{-6}$ for all values of $\alpha$ as above. I construct an approximate inverse preconditioner $(A+\sigma I)^{-1}$ with $\sigma=10^{-4}$ by using five iterations of an order 16 hyperpower iterative inversion method. The final iterate is compressed to an accuracy of $\epsilon=10^{-5}$ for the cases of $\alpha=1.1$ and 1.3 , but required a tighter accuracy of $\epsilon=10^{-6}$ for the $\alpha=1.9$ case to be an effective preconditioner. The CG solution is computed to a relative accuracy $\|A U-q\| /\|q\|$ of $10^{-10}$ using these preconditioners.

\begin{tabular}{|c|c|c|c|c|c|}
\hline$\alpha$ & $\begin{array}{c}A_{d} \text { mem } \\
(\mathrm{GB})\end{array}$ & $\begin{array}{c}A_{l r} \text { mem } \\
(\mathrm{GB})\end{array}$ & $\begin{array}{c}\text { Approx Inverse } \\
\text { LR mem (GB) }\end{array}$ & $\begin{array}{c}\text { Inversion Time } \\
(\mathrm{s})\end{array}$ & CG Iterations \\
\hline \hline 1.1 & 0.173 & 0.078 & 0.181 & 529.4 & 60 \\
\hline 1.5 & 0.173 & 0.071 & 0.134 & 478.2 & 39 \\
\hline 1.9 & 0.173 & 0.052 & 0.149 & 421.3 & 26 \\
\hline
\end{tabular}

Table 10.3: Cost of $A$ and its approximate inverse, and CG iterations, for different values of $\alpha$.

Table 10.3 shows in columns 2-4 the memory footprint of the dense and low rank 
blocks of the forward operator $A$ and of its approximate inverse used as preconditioner. The fifth column shows the time needed to generate the preconditioner on the P100 Pascal GPU. The final column of the table shows the number of CG iterations for convergence with the Gaussian pulse input used above, starting from an initial allzeros guess. Except for using a compression accuracy of $10^{-6}$ for $\alpha=1.9$ and a looser accuracy of $10^{-5}$ for $\alpha=1.1,1.5$, the hierarchical preconditioners are all quite robust across the range of the order of the fractional operator. I note that increasing $\alpha$ leads to slightly lower inversion time primarily due to slightly lower ranks in the matrix and its inverse.

The approximate inverse requires more memory than the forward operator, as the ranks of the low rank blocks increase, however the overall savings remain substantial across the values of $\alpha$. Whereas a fully dense representation of the operator or its dense inverse would require $5 \mathrm{~GB}$, the hierarchical inverse is quite compressible with a $12-15 \times$ reduction in overall memory use even for this relatively small problem with $N=25,921$. I also note that the solution time, once the preconditioner is built, is always a fraction of a second on the GPU. 


\section{Chapter 11}

\section{Conclusions}

\subsection{Contributions}

In this dissertation, I have developed a set of high performance GPU hierarchical linear algebra operations using $\mathcal{H}^{2}$-matrices that generalize standard dense linear algebra and exhibit optimal complexity in memory and optimal to near-optimal complexity in time. Matrix-vector multiplication, compression, and construction from matrix-vector product sampling algorithms are developed and analyzed in detail. To overcome the architectural challenges of attaining high performance on GPUs for tree operations involving tiny matrices, I split each hierarchical operation into a marshaling phase that generates batches of operations that can be carried out in parallel from each level of the flattened tree data and a batch phase that executes those operations in parallel using a single kernel call. To better support batched execution on the GPU, I develop a set of efficient batched linear algebra subroutines that either out-

perform the state-of-the-art routines from cuBLAS and MAGMA or are the first of their kind on the GPU, such as the batched singular value decomposition kernels and the adaptive randomized approximation. This clean separation of concerns allows the resulting library to run the same code efficiently on both CPU and GPU with a single layer of abstraction around the batched routines.

I demonstrate the performance of the library in two applications: Hessians arising in inverse problems governed by partial differential equations, where a significant reduction in the number of samples needed to approximate a Hessian compared to 
globally low rank representations is achieved, and hierarchical matrix approximations for space-fractional diffusion equations, where a reduction in the memory and computational complexity of the reference dense implementation from quadratic to linear is achieved.

Finally, I demonstrate the scalability of the hierarchical operations by developing a distributed multiGPU hierarchical matrix vector product. The resulting algorithm focuses on reducing the volume of communication and hiding both communication time and low GPU utilization at the higher levels of the tree using low priority streams. Perfect weak scalability is observed on tests up to 16 GPUs.

\subsection{Future Work}

There are many aspects of this dissertation that lead to further enhancements, additional features and in-depth investigation, such as the removal of some software limitations, the development of more efficient algorithms, more distributed operations, and numerical analysis.

My current implementation uses a fixed rank per level of the basis tree due to the lack of available high performance non-uniform batched GPU routines. This limitation also currently forces the basis tree to be a complete tree, since in practice I observe that, for many problems, blocks of approximately the same size tend to have similar ranks (with the exception of some outliers). Having a complete basis tree means that the resulting low rank blocks in the matrix tree are approximately the same size. Development of these non-uniform batched routine would remove this limitation and allow blocks of any size to appear on any level, greatly increasing the variety of represented hierarchical matrices and potentially reducing the total memory footprint of the matrix.

During the analysis of the hierarchical adaptive randomized approximation algorithm, I noted that using higher order methods that shifted the computational load to 
the sampling phase lead to better overall performance. This is due to the significant gap in performance between the compression kernels and the matrix multiplication kernels of the sampling and the need to split the low rank update into smaller updates, each requiring a costly compression. If I were able to interleave the different phases of the operations and take advantage of the special structure of the updates that are applied to the coupling matrices, i.e., the identity matrix, I could greatly increase the block size of the split low rank update. The overall algorithm would then interleave the phases of hlru, horthog, and hcompress:

- Update the basis tree (or a branch of it) with the low rank update data. The corresponding coupling nodes update will now be implicit and not actually carried out. This will lead to significant memory savings for a general structure, since the final compressed nodes should have a relatively low rank.

- Orthogonalize the basis (or a branch of it) and store the resulting projection nodes. The projection phase will not be carried out at this point.

- The basis generation phase of the compression in Section 5.3 will form the matrices $\bar{S}_{i}$ using matrix multiplication with the implicitly updated coupling nodes instead of a transpose with the explicitly formed nodes.

- The compression of the basis will then produce the projection matrices that can finally be applied to the implicitly updated coupling nodes. This final phase is where a significant portion of the computational gains can be made. Since a large update is being applied to a basis that is expected to have low ranks, the adaptive randomized approximation can be used more effectively (and the SVD can still be applied as a final post-processing step to reduce the ranks to their minimum).

An efficient implementation could see significant performance gains due to the higher 
efficiency of the ARA on larger blocks, and significantly cut down on the number of low rank updates that have to be compressed during hara.

I showed that the distributed hierarchical matrix vector product can scale quite well. Since the hgemv involves tree operations that are structurally similar to those of the other hierarchical operations, namely the upsweep, downsweep and projection, I expect distributed implementations of the remaining operations to exhibit similar scaling behavior. However, rather than implementing four additional distributed algorithms, perhaps implementing the combined method above as a single distributed algorithm would be more effective.

Throughout this dissertation, I assumed that I am given a structure for the hierarchical matrix and most presented structures were generated using standard geometric admissibility conditions. One future direction could be investigating other methods for generating structures that can adapt to and change dynamically based on the application or computation. The problems I tackled were also two or three dimensional problems and many application of interest involve higher dimensional problems where geometric data may not always be available. Finding the best structure for a hierarchical matrix is still an open problem.

Finally, careful numerical analysis will be needed for some of the hierarchical operations. For example, peeling off the upper levels during the hara algorithm introduces error in the lower levels, since the blocks that are being generated are only approximations of the blocks being peeled off. The accumulation of errors could impact the accuracy of the final result as well as cause the approximation algorithm to sample more than it needs to. This is a result of the decay of the singular values of the blocks at the lower levels being changed by the accumulating errors as well as the conservative nature of the approximation threshold used in ARA. The threshold that is used during compression is similarly impacted by this, where it must often be tuned to be lower than the ARA threshold to avoid making the error accumulation 
problem worse. One potential solution would be to compute the SVD of the generated low rank updates before applying them to more accurately control the error. This phenomenon requires more careful numerical analysis, which could lead to a more intelligent threshold selection.

\subsection{Conclusion}

The library developed in this dissertation is the first to support the high performance execution of $\mathcal{H}^{2}$-matrix operations on the GPU. The implementation of the distributed operations make it a key tool for the development of scalable hierarchical solvers for large-scale computations when integrated into existing software packages such as PETSc [134]. Thanks to the separation of the operations into marshaling and batched execution, the code is highly maintainable and future proofed. The software is available as an open source package integrated into the hierarchical computations on manycore architectures (HiCMA) library [135]. 


\section{REFERENCES}

[1] W. Hackbusch, "A sparse matrix arithmetic based on $\mathcal{H}$-matrices. Part I: Introduction to $\mathcal{H}$-matrices," Computing, vol. 62, no. 2, pp. 89-108, 1999.

[2] W. Hackbusch and B. N. Khoromskij, "A sparse $\mathcal{H}$-matrix arithmetic. Part II: Application to multi-dimensional problems," Computing, vol. 64, no. 1, pp. 21-47, 2000.

[3] W. Hackbusch and S. Börm, "Data-sparse approximation by adaptive $\mathcal{H}^{2}$ matrices," Computing, vol. 69, no. 1, pp. 1-35, 2002.

[4] S. Börm, "Approximation of integral operators by matrices with adaptive bases," Computing, vol. 74, no. 3, pp. 249-271, 2005.

[5] - "Approximation of solution operators of elliptic partial differential equations by $\mathcal{H}$ - and $\mathcal{H}^{2}$-matrices," Numerische Mathematik, vol. 115, no. 2, pp. 165-193, 2010. [Online]. Available: http://dx.doi.org/10.1007/ s00211-009-0278-7

[6] L. Grasedyck, W. Hackbusch, and B. N. Khoromskij, "Solution of large scale algebraic matrix Riccati equations by use of hierarchical matrices," Computing, vol. 70, no. 2, pp. 121-165, 2003. [Online]. Available: http://dx.doi.org/10.1007/s00607-002-1470-0

[7] L. Grasedyck, R. Kriemann, and S. Le Borne, "Domain decomposition based $\mathcal{H}$-LU preconditioning," Numerische Mathematik, vol. 112, no. 4, pp. 565-600, 2009. [Online]. Available: http://dx.doi.org/10.1007/s00211-009-0218-6

[8] W. Hackbusch, Hierarchical matrices : Algorithms and Analysis, ser. Springer series in computational mathematics. Berlin: Springer, 2015, vol. 49.

[9] M. Bebendorf, Hierarchical Matrices: A Means to Efficiently Solve Elliptic Boundary Value Problems, 1st ed. Springer Publishing Company, Incorporated, 2008.

[10] W. Hackbusch, B. Khoromskij, and S. Sauter, "On $\mathcal{H}^{2}$-matrices," in Lectures on Applied Mathematics, H. Bungartz, R. Hoppe, and Z. C., Eds. Springer, 2000, pp. 9-29. 
[11] W. Hackbusch, S. Börm, and L. Grasedyck, $\mathcal{H}$-lib. [Online]. Available: www.hlib.org

[12] S. Börm, H2-lib. [Online]. Available: www.h2lib.org

[13] R. Kriemann, $\mathcal{H}$-lib pro, 2016. [Online]. Available: www.hlibpro.com

[14] — " "H-LU factorization on many-core systems," Computing and Visualization in Science, vol. 16, no. 3, pp. 105-117, 2013.

[15] J. Xia, S. Chandrasekaran, M. Gu, and X. S. Li, "Fast algorithms for hierarchically semiseparable matrices," Numerical Linear Algebra with Applications, vol. 17, no. 6, pp. 953-976, 2010.

[16] S. Ambikasaran and E. Darve, "An o(n $\log n)$ fast direct solver for partial hierarchically semi-separable matrices," Journal of Scientific Computing, vol. 57, no. 3, pp. 477-501, 2013.

[17] A. Gillman, P. M. Young, and P.-G. Martinsson, "A direct solver with $O(N)$ complexity for integral equations on one-dimensional domains," Frontiers of Mathematics in China, vol. 7, no. 2, pp. 217-247, 2012.

[18] P. Martinsson, "A direct solver for variable coefficient elliptic PDEs discretized via a composite spectral collocation method," Journal of Computational Physics, vol. 242, pp. 460 - 479, 2013. [Online]. Available: http://www.sciencedirect.com/science/article/pii/S0021999113001320

[19] T. Mary, "Block low-rank multifrontal solvers: complexity, performance, and scalability," Ph.D. dissertation, 112017.

[20] P. Amestoy, C. Ashcraft, O. Boiteau, A. Buttari, J.-Y. L'Excellent, and C. Weisbecker, "Improving multifrontal methods by means of block low-rank representations," SIAM Journal on Scientific Computing, vol. 37, no. 3, pp. A1451A1474, 2015.

[21] P. R. Amestoy, I. S. Duff, J. Koster, and J.-Y. L'Excellent, "A fully asynchronous multifrontal solver using distributed dynamic scheduling," SIAM Journal on Matrix Analysis and Applications, vol. 23, no. 1, pp. 15-41, 2001.

[22] P. R. Amestoy, A. Guermouche, J.-Y. L'Excellent, and S. Pralet, "Hybrid scheduling for the parallel solution of linear systems," Parallel Computing, vol. 32, no. 2, pp. 136-156, 2006.

[23] K. Akbudak, H. Ltaief, A. Mikhalev, A. Charara, A. Esposito, and D. Keyes, "Exploiting data sparsity for large-scale matrix computations," in Euro-Par 
2018: Parallel Processing, M. Aldinucci, L. Padovani, and M. Torquati, Eds. Cham: Springer International Publishing, 2018, pp. 721-734.

[24] L. Lin, J. Lu, and L. Ying, "Fast construction of hierarchical matrix representation from matrix-vector multiplication," Journal of Computational Physics, vol. 230, no. 10, pp. 4071-4087, 2011.

[25] P. Martinsson, "Compressing rank-structured matrices via randomized sampling," SIAM Journal on Scientific Computing, vol. 38, no. 4, pp. A1959-A1986, 2016.

[26] — - "A fast randomized algorithm for computing a hierarchically semiseparable representation of a matrix," SIAM Journal on Matrix Analysis and Applications, vol. 32, no. 4, pp. 1251-1274, 2011.

[27] J. Xia, "Randomized sparse direct solvers," SIAM Journal on Matrix Analysis and Applications, vol. 34, no. 1, pp. 197-227, 2013.

[28] C. D. Yu, J. Levitt, S. Reiz, and G. Biros, "Geometry-oblivious FMM for compressing dense SPD matrices," in Proceedings of the International Conference for High Performance Computing, Networking, Storage and Analysis, ser. SC '17, 2017, pp. 53:1-53:14.

[29] C. D. Yu, S. Reiz, and G. Biros, "Distributed-memory hierarchical compression of dense SPD matrices," in Proceedings of the International Conference for High Performance Computing, Networking, Storage, and Analysis, ser. SC '18, 2018, pp. 15:1-15:15.

[30] S. Börm and S. Christophersen, "Approximation of BEM matrices using GPGPUs," arXiv, vol. abs/1510.07244, 2015.

[31] P. Zaspel, "Algorithmic patterns for $\mathcal{H}$-matrices on many-core processors," CoRR, vol. abs/1708.09707, 2017. [Online]. Available: http://arxiv.org/abs/ 1708.09707

[32] I. Yamazaki, A. Abdelfattah, A. Ida, S. Ohshima, S. Tomov, R. Yokota, and J. Dongarra, "Analyzing performance of BiCGSTAB with hierarchical matrix on GPU clusters," in IEEE International Parallel and Distributed Processing Symposium (IPDPS), IEEE. Vancouver, BC, Canada: IEEE, 2018-05 2018.

[33] S. Ohshima, I. Yamazaki, A. Ida, and R. Yokota, "Optimization of numerous small dense-matrix-vector multiplications in $\mathcal{H}$-matrix arithmetic on GPU," in 2019 IEEE 13th International Symposium on Embedded Multicore/Many-core Systems-on-Chip (MCSoC), Oct 2019, pp. 9-16. 
[34] D. Keyes, H. Ltaief, and G. Turkiyyah, "Hierarchical algorithms on hierarchical architectures," Philosophical Transactions of the Royal Society A, vol. 378, p. 20190055, 2019.

[35] A. Haidar, T. T. Dong, S. Tomov, P. Luszczek, and J. Dongarra, "A framework for batched and GPU-resident factorization algorithms applied to block Householder transformations." in International Supercomputing Conference, ser. Lecture Notes in Computer Science, J. M. Kunkel and T. Ludwig, Eds., vol. 9137. Springer, 2015, pp. 31-47.

[36] A. Haidar, T. Dong, P. Luszczek, S. Tomov, and J. Dongarra, "Optimization for performance and energy for batched matrix computations on GPUs," in Proceedings of the 8th Workshop on General Purpose Processing Using GPUs, ser. GPGPU-8. New York, NY, USA: ACM, 2015, pp. 59-69.

[37] N. Wilt, The CUDA Handbook: A Comprehensive Guide to GPU Programming. Pearson Education, 2013.

[38] V. Volkov, "Better performance at lower occupancy," Proceedings of the GPU technology conference, GTC, vol. 10, 2010.

[39] G. Golub and C. Van Loan, Matrix Computations. Johns Hopkins University Press, 2013.

[40] L. Trefethen and D. Bau, Numerical Linear Algebra. Society for Industrial and Applied Mathematics, 1997.

[41] J. Demmel and K. Veselic, "Jacobi's method is more accurate than QR," SIAM Journal on Matrix Analysis and Applications, vol. 13, no. 4, pp. 1204-1245, 1992.

[42] N. Halko, P.-G. Martinsson, and J. A. Tropp, "Finding structure with randomness: Probabilistic algorithms for constructing approximate matrix decompositions," SIAM Review, vol. 53, no. 2, pp. 217-288, 2011.

[43] W. H. Boukaram, G. Turkiyyah, H. Ltaief, and D. E. Keyes, "Batched QR and SVD algorithms on GPUs with applications in hierarchical matrix compression," Parallel Computing, vol. 74, pp. 19-33, 2018.

[44] J. W. Daniel, W. B. Gragg, L. Kaufman, and G. W. Stewart, "Reorthogonalization and stable algorithms for updating the Gram-Schmidt QR factorization," Mathematics of Computation, vol. 30, no. 136, pp. 772-795, 1976.

[45] G. W. Stewart, "Block Gram-Schmidt orthogonalization," SIAM Journal on Scientific Computing, vol. 31, no. 1, pp. 761-775, 2008. 
[46] S. Tomov, R. Nath, H. Ltaief, and J. Dongarra, "Dense linear algebra solvers for multicore with GPU accelerators," in Proceedings of the IEEE IPDPS'10. Atlanta, GA: IEEE Computer Society, April 19-23 2010, pp. 1-8, DOI: 10.1109/IPDPSW.2010.5470941.

[47] NVIDIA, cuBLAS Library User Guide, v8.0 ed., http://docs.nvidia.com/cuda/cublas, NVIDIA, 2017. [Online]. Available: http://docs.nvidia.com/cuda/cublas

[48] M. Anderson, G. Ballard, J. Demmel, and K. Keutzer, "Communicationavoiding QR decomposition for GPUs," in Parallel Distributed Processing Symposium (IPDPS), 2011 IEEE International, May 2011, pp. 48-58.

[49] J. Cheng, M. Grossman, and T. McKercher, Professional CUDA C Programming, ser. EBL-Schweitzer. Wiley, 2014.

[50] J. Kurzak, H. Ltaief, J. Dongarra, and R. M. Badia, "Scheduling dense linear algebra operations on multicore processors," Concurrency and Computation: Practice and Experience, vol. 22, no. 1, pp. 15-44, 2010. [Online]. Available: http://dx.doi.org/10.1002/cpe.1467

[51] B. B. Zhou and R. P. Brent, "On parallel implementation of the one-sided Jacobi algorithm for singular value decompositions," in Parallel and Distributed Processing, 1995. Proceedings. Euromicro Workshop on, Jan 1995, pp. 401-408.

[52] B. Zhou and R. Brent, "A parallel ring ordering algorithm for efficient one-sided Jacobi SVD computations," Journal of Parallel and Distributed Computing, vol. 42, no. 1 , pp. 1 - 10, 1997.

[53] M. Bečka and M. Vajteršic, "Block-Jacobi SVD algorithms for distributed memory systems I: Hypercubes and rings*," Parallel Algorithms and Applications, vol. 13, no. 3, pp. 265-287, 1999.

[54] — , "Block-Jacobi SVD algorithms for distributed memory systems II: Meshes*," Parallel Algorithms and Applications, vol. 14, no. 1, pp. 37-56, 1999.

[55] M. Bečka, G. Okša, and M. Vajteršic, "New dynamic orderings for the parallel one-sided block-Jacobi SVD algorithm," Parallel Processing Letters, vol. 25, no. 02 , p. $1550003,2015$.

[56] NVIDIA, cuSOLVER Library User Guide, v8.0 ed., http://docs.nvidia.com/cuda/cusolver/, NVIDIA, 2017. [Online]. Available: http://docs.nvidia.com/cuda/cusolver 
[57] G. Okša and M. Vajteršic, "Efficient pre-processing in the parallel block-Jacobi SVD algorithm," Parallel Computing, vol. 32, no. 2, pp. 166-176, Feb. 2006. [Online]. Available: http://dx.doi.org/10.1016/j.parco.2005.06.006

[58] P. Martinsson and S. Voronin, "A randomized blocked algorithm for efficiently computing rank-revealing factorizations of matrices," SIAM Journal on Scientific Computing, vol. 38, no. 5, pp. S485-S507, 2016.

[59] A. Stathopoulos and K. Wu, "A block orthogonalization procedure with constant synchronization requirements," SIAM Journal on Scientific Computing, vol. 23, no. 6, pp. 2165-2182, 2002.

[60] I. Yamazaki, S. Tomov, and J. Dongarra, "Mixed-precision Cholesky QR factorization and its case studies on multicore CPU with multiple GPUs," SIAM Journal on Scientific Computing, vol. 37, no. 3, pp. C307-C330, 2015.

[61] Y. Hida, X. S. Li, and D. H. Bailey, "Algorithms for quad-double precision floating point arithmetic," in Computer Arithmetic, 2001. Proceedings. 15th IEEE Symposium on. IEEE, 2001, pp. 155-162.

[62] M. Lu, B. He, and Q. Luo, "Supporting extended precision on graphics processors," in Proceedings of the Sixth International Workshop on Data Management on New Hardware. ACM, 2010, pp. 19-26.

[63] R. Nath, S. Tomov, and J. Dongarra, "Accelerating GPU kernels for dense linear algebra," in Proceedings of the 2009 International Meeting on High Performance Computing for Computational Science, VECPAR'10. Berkeley, CA: Springer, June 22-25 2010.

[64] NVIDIA, CURAND LIBRARY Programming Guide, v8.0 ed., NVIDIA, 2018. [Online]. Available: https://docs.nvidia.com/cuda/curand

[65] R. Yokota, G. Turkiyyah, and D. Keyes, "Communication Complexity of the Fast Multipole Method and Its Algebraic Variants," Supercomputing Frontiers and Innovations: an International Journal, vol. 1, no. 1, pp. 63-84, Apr. 2014. [Online]. Available: http://dx.doi.org/10.14529/jsfi140104

[66] S. Ambikasaran, D. Foreman-Mackey, L. Greengard, D. W. Hogg, and M. O'Neil, "Fast direct methods for Gaussian processes," IEEE Transactions on Pattern Analysis \& Machine Intelligence, vol. 38, no. 2, pp. 252-265, 2016.

[67] W. Hackbusch, Hierarchical Matrices: Algorithms and Analysis. Springer, 2015. 
[68] S. Börm, M. Löhndorf, and J. M. Melenk, "Approximation of integral operators by variable-order interpolation," Numerische Mathematik, vol. 99, no. 4, pp. 605-643, 2005.

[69] L. Grasedyck and W. Hackbusch, "Construction and arithmetics of $\mathcal{H}$ matrices," Computing, vol. 70, no. 4, pp. 295-334, 2003.

[70] A. Abdelfattah, D. Keyes, and H. Ltaief, "Kblas: An optimized library for dense matrix-vector multiplication on gpu accelerators," ACM Transactions on Mathematical Software, vol. 42, no. 3, pp. 18:1-18:31, 2016.

[71] A. Abdelfattah, H. Ltaief, D. Keyes, and J. Dongarra, "Performance optimization of sparse matrix-vector multiplication for multi-component PDE-based applications using gpus," Concurrency and Computation: Practice and Experience, vol. 28, no. 12, pp. 3447-3465, 2016.

[72] NVIDIA, cuSPARSE Library Documentation (v8.0), 2016.

[73] S. Börm, Efficient numerical methods for non-local operators: $\mathcal{H}^{2}$-matrix compression, algorithms and analysis. European Mathematical Society, 2010, vol. 14 .

[74] F. Rouet, X. Li, P. Ghysels, and A. Napov, "A distributed-memory package for dense hierarchically semi-separable matrix computations using randomization," ACM Transactions on Mathematical Software, vol. 42, no. 4, pp. 27:1-27:35, 2016 .

[75] P. Ghysels, X. Li, F. Rouet, S. Williams, and A. Napov, "An efficient multicore implementation of a novel HSS-structured multifrontal solver using randomized sampling," SIAM Journal on Scientific Computing, vol. 38, no. 5, pp. S358S384, 2016.

[76] A. R. Curtis, M. J. D. Powell, and J. K. Reid, "On the estimation of sparse Jacobian matrices," IMA Journal of Applied Mathematics, vol. 13, no. 1, pp. 117-119, 1974.

[77] T. F. Coleman, B. S. Garbow, and J. J. More, "Software for estimating sparse Jacobian matrices," ACM Transactions on Mathematical Software, vol. 10, no. 3, pp. 329-345, 1984.

[78] T. F. Coleman, B. S. Garbow, and J. J. Moré, "Software for estimating sparse Hessian matrices," ACM Transactions on Mathematical Software, vol. 11, no. 4, pp. 363-377, 1985. 
[79] A. Gebremedhin, F. Manne, and A. Pothen, "What color is your Jacobian? Graph coloring for computing derivatives," SIAM Review, vol. 47, no. 4, pp. 629-705, 2005.

[80] S. Börm and K. Reimer, "Efficient arithmetic operations for rank-structured matrices based on hierarchical low-rank updates," Computing and Visualization in Science, vol. 16, no. 6, pp. 247-258, 2013.

[81] W. Boukaram, G. Turkiyyah, and D. Keyes, "Hierarchical matrix operations on GPUs: Matrix-vector multiplication and compression," ACM Transactions on Mathematical Software, vol. 45, no. 1, pp. 3:1-3:28, Feb. 2019.

[82] S. Börm, "Data-sparse approximation of non-local operators by $\mathcal{H}^{2}$-matrices," Linear Algebra and its Applications, vol. 422, no. 2, pp. 380 - 403, 2007.

[83] S. Hao and P.-G. Martinsson, "A direct solver for elliptic PDEs in three dimensions based on hierarchical merging of Poincaré-Steklov operators," Journal of Computational and Applied Mathematics, vol. 308, pp. 419 - 434, 2016.

[84] G. Schulz, "Iterative Berechung der reziproken Matrix," ZAMM - Journal of Applied Mathematics and Mechanics / Zeitschrift für Angewandte Mathematik und Mechanik, vol. 13, no. 1, pp. 57-59, 1933.

[85] T. Söderström and G. Stewart, "On the numerical properties of an iterative method for computing the moore-penrose generalized inverse," SIAM Journal on Numerical Analysis, vol. 11, no. 1, pp. 61-74, 1974.

[86] P. Sanders, J. Speck, and R. Steffen, "Work-efficient matrix inversion in polylogarithmic time," ACM Transactions on Parallel Computing, vol. 2, no. 3, pp. 15:1-15:29, Sep. 2015.

[87] W. Boukaram, G. Turkiyyah, and D. Keyes, "Randomized GPU algorithms for the construction of hierarchical matrices from mat-vec operations," SIAM Journal on Scientific Computing, vol. 41(4): C339-C366, 2019.

[88] V. Pan and R. Schreiber, "An improved Newton iteration for the generalized inverse of a matrix, with applications," SIAM Journal on Scientific Computing, vol. 12, no. 5, pp. 1109-1130, 1991.

[89] W. Hackbusch, B. N. Khoromskij, and E. E. Tyrtyshnikov, "Approximate iterations for structured matrices," Numerische Mathematik, vol. 109, no. 3, pp. 365-383, May 2008. 
[90] V. Pan, F. Soleymani, and L. Zhao, "An efficient computation of generalized inverse of a matrix," Applied Mathematics and Computation, vol. 316, pp. $89-$ $101,2018$.

[91] M. Heinkenschloss, "Mesh independence for nonlinear least squares problems with norm constraints," SIAM Journal on Optimization, vol. 3, pp. 81-117, 1993.

[92] I. Epanomeritakis, V. Akçelik, O. Ghattas, and J. Bielak, "A Newton-CG method for large-scale three-dimensional elastic full-waveform seismic inversion," Inverse Problems, vol. 24, no. 3, p. 034015 (26pp), 2008.

[93] T. Isaac, N. Petra, G. Stadler, and O. Ghattas, "Scalable and efficient algorithms for the propagation of uncertainty from data through inference to prediction for large-scale problems, with application to flow of the Antarctic ice sheet," Journal of Computational Physics, vol. 296, pp. 348-368, September 2015 .

[94] A. Alexanderian, P. J. Gloor, and O. Ghattas, "On Bayesian A-and D-optimal experimental designs in infinite dimensions," Bayesian Analysis, vol. 11, no. 3, pp. 671-695, 2016, arXiv preprint arXiv:1408.6323.

[95] M. Bebendorf and W. Hackbusch, "Existence of $\mathcal{H}$-matrix approximants to the inverse FE-matrix of elliptic operators with $L^{\infty}$-coefficients," Numerische Mathematik, vol. 95, no. 1, pp. 1-28, 2003.

[96] Y. Yang and B. Engquist, "Analysis of optimal transport and related misfit functions in full-waveform inversion," Geophysics, vol. 83, no. 1, pp. A7-A12, 2018 .

[97] T. Kolda and B. Bader, "Tensor decompositions and applications," SIAM Review, vol. 51, no. 3, pp. 455-500, 2009.

[98] M. Bebendorf, "Low-rank approximation of elliptic boundary value problems with high-contrast coefficients," SIAM Journal on Mathematical Analysis, vol. 48, no. 2, pp. 932-949, 2016.

[99] S. Börm, "Directional $\mathcal{H}^{2}$-matrix compression for high-frequency problems," Numerical Linear Algebra with Applications, vol. 24, no. 6, p. e2112, 2017.

[100] M. Hinze, R. Pinnau, M. Ulbrich, and S. Ulbrich, Optimization with PDE Constraints. Springer, 2009.

[101] Y. Wang, "Frequencies of the Ricker wavelet," GEOPHYSICS, vol. 80, no. 2, pp. A31-A37, 2015. [Online]. Available: https://doi.org/10.1190/geo2014-0441.1 
[102] D. N. Arnold, F. Brezzi, B. Cockburn, and L. D. Marini, "Unified analysis of discontinuous Galerkin methods for elliptic problems," SIAM Journal on Numerical Analysis, vol. 39, no. 5, pp. 1749-1779, 2002.

[103] B. Riviere, Discontinuous Galerkin methods for solving elliptic and parabolic equations: theory and implementation. SIAM, 2008.

[104] B. Ayuso and L. D. Marini, "Discontinuous Galerkin methods for advectiondiffusion-reaction problems," SIAM Journal on Numerical Analysis, vol. 47, no. 2, pp. 1391-1420, 2009.

[105] A. Logg, K.-A. Mardal, G. N. Wells et al., Automated Solution of Differential Equations by the Finite Element Method. Springer, 2012.

[106] U. Villa, N. Petra, and O. Ghattas, "hIPPYlib: an Extensible Software Framework for Large-scale Deterministic and Bayesian Inverse Problems," Journal of Open Source Software, vol. 3, no. 30, 2018.

[107] Q. Du, Nonlocal Modeling, Analysis, and Computation. SIAM, 2019.

[108] J. Klafter and I. M. Sokolov, "Anomalous diffusion spreads its wings," Physics World, vol. 18, no. 8, p. 29, 2005.

[109] P. Gatto and J. S. Hesthaven, "Numerical approximation of the fractional laplacian via $h p$-finite elements, with an application to image denoising," Journal of Scientific Computing, vol. 65, no. 1, pp. 249-270, 2015.

[110] S. G. Kou, "A jump-diffusion model for option pricing," Management Science, vol. 48, no. 8, pp. 1086-1101, 2002.

[111] C. Li and M. Cai, Theory and Numerical Approximations of Fractional Integrals and Derivatives. SIAM, 2020.

[112] M. Kwasnicki, "Ten equivalent definitions of the fractional Laplace operator," Fractional Calculus and Applied Analysis, vol. 20, no. 1, pp. 7-51, 2017.

[113] C. Tadjeran, M. M. Meerschaert, and H.-P. Scheffler, "A second-order accurate numerical approximation for the fractional diffusion equation," Journal of Computational Physics, vol. 213, no. 1, pp. 205-213, 2006.

[114] W. Deng and J. S. Hesthaven, "Local Discontinuous Galerkin methods for fractional diffusion equations," ESAIM: Mathematical Modelling and Numerical Analysis, vol. 47, no. 6, pp. 1845-1864, 2013.

[115] K. Mustapha, "Time-stepping discontinuous Galerkin methods for fractional diffusion problems," Numerische Mathematik, vol. 130, no. 3, pp. 497-516, 2015. 
[116] R. Gorenflo, A. Vivoli, and F. Mainardi, "Discrete and continuous random walk models for space-time fractional diffusion," Nonlinear Dynamics, vol. 38, no. 1, pp. 101-116, 2004.

[117] H. Zhang, F. Liu, and V. Anh, "Numerical approximation of Lévy-Feller diffusion equation and its probability interpretation," Journal of Computational and Applied Mathematics, vol. 206, no. 2, pp. 1098-1115, 2007.

[118] Y. Luchko, "A new fractional calculus model for the two-dimensional anomalous diffusion and its analysis," Mathematical Modeling of Natural Phenomena, vol. 11, no. 3, pp. 1-17, 2016.

[119] M. Lucchesi, S. Allouch, O. Le Maître, K. Mustapha, and O. Knio, "Particle simulation of space-fractional diffusion equations," Computational Particle Mechanics, 2019. [Online]. Available: https://hal.archives-ouvertes.fr/ hal-02327700

[120] V. Minden and L. Ying, "A simple solver for the fractional laplacian in multiple dimensions," SIAM Journal on Scientific Computing, vol. 42, no. 2, pp. A878-A900, 2020. [Online]. Available: https://doi.org/10.1137/18M1170406

[121] X. Zhao, X. Hu, W. Cai, and G. E. Karniadakis, "Adaptive finite element method for fractional differential equations using hierarchical matrices," Computer Methods in Applied Mechanics and Engineering, vol. 325, pp. 56-76, 2017.

[122] M. Karkulik and J. M. Melenk, "H-H-matrix approximability of inverses of discretizations of the fractional Laplacian," Advanced in Computational Mathematics, vol. 46, 2019.

[123] K. Xu and E. Darve, "Efficient numerical method for models driven by Lévy process via hierarchical matrices," 2018.

[124] C. Pozrikidis, The Fractional Laplacian. Chapman and Hall/CRC, 2016.

[125] A. J. Chorin, "Numerical study of slightly viscous flow," Journal of Fluid Mechanics, vol. 57, no. 4, pp. 785-796, 1973.

[126] A. Leonard, "Vortex methods for flow simulation," Journal of Computational Physics, vol. 37, no. 3, pp. 289-335, 1980.

[127] G.-H. Cottet and P. D. Koumoutsakos, Vortex methods: theory and practice. Cambridge University Press, 2000. 
[128] J. T. Beale and A. Majda, "High order accurate vortex methods with explicit velocity kernels," Journal of Computational Physics, vol. 58, no. 2, pp. 188-208, 1985.

[129] J. D. Eldredge, A. Leonard, and T. Colonius, "A general deterministic treatment of derivatives in particle methods," Journal of Computational Physics, vol. 180, no. 2, pp. 686-709, 2002.

[130] K. B. Oldham, J. Myland, and J. Spanier, An atlas of functions: with Equator, the atlas function calculator. Springer Science \& Business Media, 2010.

[131] J. W. Pearson, "Computation of hypergeometric functions," Ph.D. dissertation, University of Oxford, 2009.

[132] S. Börm, Efficient numerical methods for non-local operators: $\mathcal{H}^{2}$-matrix compression, algorithms and analysis. European Mathematical Society, 2010, vol. 14 .

[133] V. J. Ervin, N. Heuer, and J. P. Roop, "Regularity of the solution to 1-d fractional order diffusion equations," Mathematics of Computation, vol. 87, pp. 2273-2294, 2018.

[134] S. Balay et al., "PETSc users manual: Revision 3.10," Argonne National Lab.(ANL), Argonne, IL (United States), Tech. Rep., 2018.

[135] Abdulah S, Akbudak K, Boukaram W, Charara A, Keyes D, Ltaief H, Mikhalev A, Sukkari D, Turkiyyah G., Hierarchical computations on manycore architectures (HiCMA), http://github.com/ecrc/hicma, KAUST, 2019. [Online]. Available: http://github.com/ecrc/hicma 


\section{APPENDICES}

\section{A Software and External Dependencies}

The library developed in this thesis will be available as an open source package on the Extreme Computing Research Center (ECRC) github repository. The library provides routines for hierarchical matrix-vector multiplication, basis orthogonalization, compression, low rank updates, and construction using black box matrix-vector

products. It runs on both CPUs and GPUs and test codes for all operations are provided. The CPU-only installation has the following external dependencies:

- Thrust: a template $\mathrm{C}++$ parallel programming library that enables performance portability between GPUs and multicore CPUs. https://github.com/ thrust/thrust

- Any BLAS and LAPACK library such as OpenBLAS or the Intel Math Kernel Library.

The GPU installation requires the following additional dependencies:

- The Matrix Algebra on GPU and Multicore Architectures (MAGMA) library, for its collection of non-uniform batched GPU kernels. https://icl.cs.utk. edu/magma/

- The KAUST BLAS (KBLAS) library, available at the ECRC github repository, for the kernels that were developed in this thesis and added to the library as 
part of its separation of concerns. These include all the operations discussed in Chapter 3. https://github.com/ecrc/kblas-gpu

\section{B Papers Submitted and Under Preparation}

- Wajih Boukaram, Stefano Zampini, Lisandro Dalcin, George Turkiyyah, and David Keyes. Distributed Multi-GPU Hierarchical Matrix-Vector Multiplication. The International Conference for High Performance Computing, Networking, Storage, and Analysis (SC20), submitted

- Wajih Boukaram, Marco Lucchesi, George Turkiyyah, Olivier Le Maitre, Omar Knio, and David Keyes. Hierarchical matrix approximations for space-fractional diffusion equations. Computer Methods in Applied Mechanics and Engineering, in revision

- Ilona Ambartsumyan, Wajih Boukaram, Tan Bui-Thanh, Omar Ghattas, David Keyes, Georg Stadler, George Turkiyyah, and Stefano Zampini. Hierarchical matrix approximations of Hessians arising in inverse problems governed by pdes. SIAM Journal on Scientific Computing, in revision

- Wajih Boukaram, George Turkiyyah, and David Keyes. Randomized GPU algorithms for the construction of hierarchical matrices from mat-vec operations. SIAM Journal on Scientific Computing, vol. 41(4): C339-C366, 2019

- Wajih Boukaram, George Turkiyyah, and David Keyes. Hierarchical matrix operations on GPUs: Matrix-vector multiplication and compression. ACM Transactions on Mathematical Software, 45(1):3:1-3:28, February 2019

- Wajih Halim Boukaram, George Turkiyyah, Hatem Ltaief, and David Keyes. 
Batched QR and SVD algorithms on GPUs with applications in hierarchical matrix compression. Parallel Computing, 74:19-33, 2018 